CALIFORNIA

ENERGY

COMMISSION

\title{
DEVELOPMENT AND EVALUATION OF FULLY AUTOMATED DEMAND RESPONSE IN LARGE FACILITIES
}

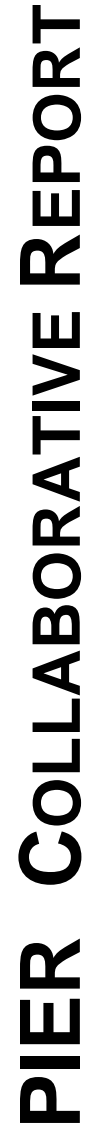




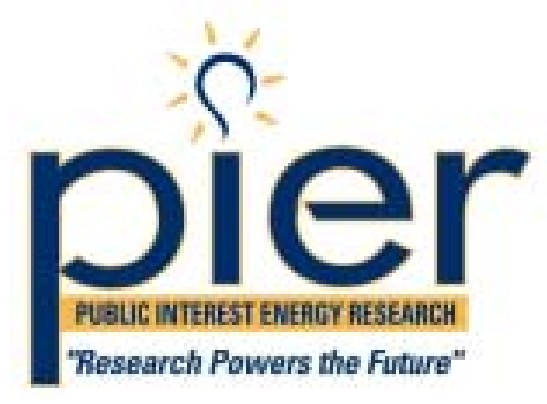

Prepared By: Mary Ann Piette Lawrence Berkeley National Laboratory

Berkeley, California

Contract No. 150-99-003

\section{Prepared For:}

\section{California Energy Commission}

Public Interest Energy Research (PIER) Program

Laurie tenHope

Contract Manager

Laurie tenHope

Program Area Team Lead

Ron Kukulka,

Acting Deputy Director

ENERGY RESEARCH AND DEVELOPMENT

DIVISION

\section{Robert L. Therkelsen}

\section{Executive Director}

The work described in this report was coordinated by the Consortium for Electric Reliability Technology

Solutions and funded by the California Energy Commission, Public Interest Energy Research Program, under Work for Others Contract No. 150-99003, Am \#1 and by the U.S. Department of Energy under Contract No. DE-AC03-76SF00098.

\section{DISCLAIMER}

This report was prepared as the result of work sponsored by the California Energy Commission. It does not necessarily represent the views of the Energy Commission, its employees or the State of California. The Energy Commission, the State of California, its employees, contractors and subcontractors make no warrant, express or implied, and assume no legal liability for the information in this report; nor does any party represent that the uses of this information will not infringe upon privately owned rights. This report has not been approved or disapproved by the California Energy Commission nor has the California Energy Commission passed upon the accuracy or adequacy of the information in this report. 


\section{PREFACE}

The Public Interest Energy Research (PIER) Program supports public interest energy research and development that will help improve the quality of life in California by bringing environmentally safe, affordable, and reliable energy services and products to the marketplace.

The PIER Program, managed by the California Energy Commission (Commission), annually awards up to $\$ 62$ million to conduct the most promising public interest energy research by partnering with Research, Development, and Demonstration (RD\&D) organizations, including individuals, businesses, utilities, and public or private research institutions.

- $\quad$ PIER funding efforts are focused on the following six RD\&D program areas:

- Buildings End-Use Energy Efficiency

- Industrial/Agricultural/Water End-Use Energy Efficiency

- Renewable Energy

- Environmentally-Preferred Advanced Generation

- Energy-Related Environmental Research

- $\quad$ Strategic Energy Research.

What follows is the final report for the Building Vulnerability Guide, 500-01-034, conducted by the Lawrence Berkeley National Laboratory. The report is entitled Building Vulnerability Assessment and Mitigation. This project contributes to the Buildings Program.

For more information on the PIER Program, please visit the Commission's Web site at: http://www.energy.ca.gov/research/index.html or contact the Commission's Publications Unit at 916-654-5200. 


\section{$\underline{\text { Full LBNL Disclaimer }}$}

\section{DISCLAIMER}

This document was prepared as an account of work sponsored by the United States Government. While this document is believed to contain correct information, neither the United States Government nor any agency thereof, nor The Regents of the University of California, nor any of their employees, makes any warranty, express or implied, or assumes any legal responsibility for the accuracy, completeness, or usefulness of any information, apparatus, product, or process disclosed, or represents that its use would not infringe privately owned rights. Reference herein to any specific commercial product, process, or service by its trade name, trademark, manufacturer, or otherwise, does not necessarily constitute or imply its endorsement, recommendation, or favoring by the United States Government or any agency thereof, or The Regents of the University of California. The views and opinions of authors expressed herein do not necessarily state or reflect those of the United States Government or any agency thereof, or The Regents of the University of California.

Ernest Orlando Lawrence Berkeley National Laboratory is an equal opportunity employer.

Abbreviated LBNL Disclaimer (place under CEC disclaimer)

This work was also supported by the Assistant Secretary for Energy Efficiency and Renewable Energy, Building Technologies Program, of the US Department of Energy under Contract No. DE-AC03-76SF00098. 


\title{
Development and Evaluation of Fully Automated Demand Response In Large Facilities
}

\author{
March 30, 2004
}

Mary Ann Piette

Osman Sezgen

David S. Watson

Naoya Motegi

Lawrence Berkeley National Laboratory

Christine Shockman

Shockman Consulting

Laurie ten Hope

Program Manager, Energy Systems Integration

Sponsored by the California Energy Commission 



\section{Table of Contents}

Table of Contents ............................................................................................................................. i

List of Tables and Figures ........................................................................................................ii

Acknowledgements ....................................................................................................................... v

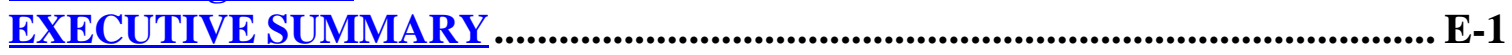

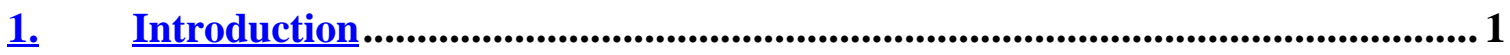

1-1 Background and Report Overview ....................................................... 1

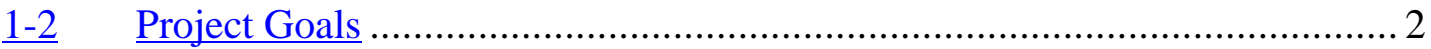

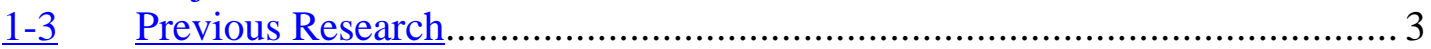

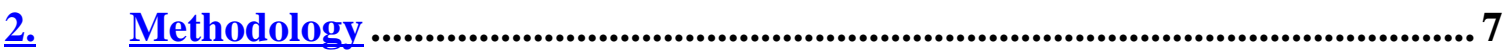

$2-1 \quad$ Project Overview and Timeline ........................................................... 7

$\underline{2-2} \quad$ Selection Criteria and Sites Considered..................................................... 8

2-3 Site Characteristics and Background ..................................................... 9

2-4 Automated Demand Response System Description .................................... 15

2-5 Controls and Communications Upgrades …………….............................. 18

2-6 Electric Price Signal and Test Description ………................................... 19

2-7 Assessment of Lead Users of Auto-DR ................................................... 20

3. Auto-DR Systems Characterization and Measurement .................................... 23

3-1 Auto-DR System Architecture ……………………………………. 23

3-2 Site Measurement and Evaluation Techniques …………….......................... 44

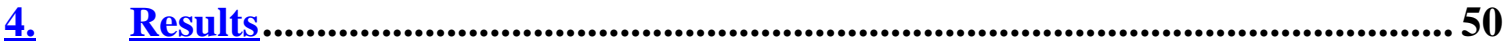

4-1 Requesting and Confirming Receipt of Signal.........................................5 50

4-2 Demand Shed Savings Estimates ............................................................. 50

5. Discussion: Organizational and Technological Issues.......................................72

5-1 Implementation and Organizational Challenges .........................................72

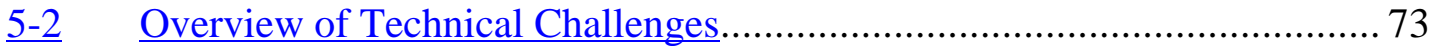

5-3 Lessons from each Site ....................................................................... 74

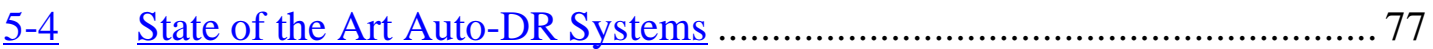

6. Summary and Future Research Topics................................................................... 80

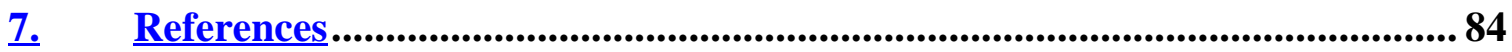

\section{Appendix I.}

Appendix II.

Appendix II-1.

Appendix III. Appendix III-1. Appendix III-2. Appendix III-3. Appendix III-4.

\section{APPENDICES} Acronyms and Terminology.............................................................I-1

Methodology - Additional Details ............................................. II-1 Whole-Building Level Method, Additional Detail .................... II-1

Sample Memorandum of Understanding ................................III-1

Project Participant Memorandum of Understanding ................. III-1

Automated Demand Response Test Questionnaire.................... III-4 Time Schedule for Demand Response Test Participants........... III-6 Price Signal for the Automated Demand Responses Tests........ III-7 
Appendix III-5.

RT Pricing Web Methods and XML Schema

III-10

Appendix IV.

Participants List

IV-1

Appendix V.

Site Questionnaires (Test 1, Test 2) .................................... V-1

Appendix V-1.

Appendix V-2.

Appendix V-3.

Appendix V-4.

Appendix V-5.

Appendix VI.

Appendix VI-1.

Appendix VI-2.

Site Questionnaires - Albertsons ........................................ V-1

Site Questionnaires - Bank of America ............................... V-3

Site Questionnaires - GSA Oakland ...................................... V-5

Site Questionnaires - Roche .............................................. V-8

Site Questionnaires - UCSB ........................................ V-10

Appendix VI-3.

Appendix VI-4.

Site Data Collection Points List ............................................ VI-1

Albertsons - Point List ..................................................... VI-1

Bank of America - Point List .............................................. VI-1

GSA Oakland - Point List ............................................... VI-3

Roche - Point List and Fan Spot Measurement Plan ............... VI-5

Appendix VI-5.

UCSB - Shed Strategies and Point List .............................. VI-9

Appendix VII.

Additional Result Tables and Figures .............................. VII-1

Appendix VII-1. $\quad$ First Day Test Results ............................................... VII-1

Appendix VII-2. $\quad$ B of A - Result Analysis by WebGen............................... VII-5

Appendix VII-3. GSA - Additional Figures ................................................. VII-7

Appendix VII-4. $\quad$ Roche - Additional Figures .............................................. VII-17

Appendix VII-5. $\quad$ UCSB - Additional Figures .......................................... VII-19

Appendix VIII. Previous DR Participation and Site Contact .....................VIII-1

Appendix VIII-1. Prior Demand Response Program Participation .................... VIII-1

Appendix VIII-2. Interaction with Site Contacts ............................................ VIII-4

Appendix VIII-3. Problems Encountered During Test Period ......................... VIII-8

\section{List of Tables and Figures}

Table 2-1. $\quad$ Project Timeline and Milestones ...................................................... 7

Table 2-2. $\quad$ Summary of Sites......................................................................... 10

Table 3-1. Characteristics of Auto-DR Systems - Architecture .............................. 24

Table 3-2. Characteristics of Auto-DR Systems - Gateways................................... 25

Table 3-3. Characteristics of Auto-DR Systems - Integration ................................. 28

Table 3-4. $\quad$ Characteristics of Auto-DR Systems - Shed Control................................29

Table 3-5. Characteristics of Auto-DR Systems - Open Standards ......................... 30

Table 3-6. Oakland GSA Zone Temperature Setpoints - Normal and Shed Modes .. 37

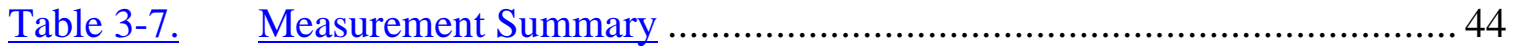

Table 3-8. Component Level Methods for Each Component ................................... 48

Table 4-1. $\quad$ Record of Prices Being Requested and Receipt Being Confirmed .......... 50

Table 4-2. Shed Strategies and Results of Response ........................................... 51

Table 4-3. UCSB -Estimated Savings by Component (Component Method)........... 65

Table 4-4. $\quad$ Summary of Estimated Savings of All Site (Whole-Building Method) ... 68 
Figure E-1.

Fictitious Electric Price Signal Sent to Five Facilities.............................. 2

Figure E-2.

Geographical Location of Auto-DR Infrastructure.................................... 3

Figure E-3. Auto-DR Electric Load Shed from five sites on Wed. Nov. 19, 2003. ...... 3

Figure E-4. Auto-DR Electric Load Shed from five sites on Wed. Nov. 19, 2003. ...... 4

Figure 1-1. Overlap Between Energy Information Systems (EIS), Energy Management Control Systems (EMCS) and Demand Response Systems (DRS)............ 5

Figure 2-1. $\quad$ Albertsons...................................................................................... 11

Figure 2-2. $\quad$ Bank of America - Concord Data Center, Building B …………............. 12

Figure 2-3. $\quad$ GSA Oakland Federal Building ........................................................... 12

Figure 2-4. $\quad$ Roche Palo Alto - Building FS (left) and A2 (right).............................. 13

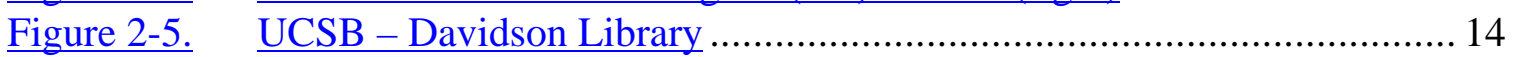

Figure 2-6. Auto-DR Network Communications Sequence......................................... 15

Figure 2-7. Geographical Location of Auto-DR Infrastructure................................... 16

Figure 2-8. $\quad$ Test 1 Price Signal (November 12 $\left.2^{\text {th }}, 2003\right)$.......................................... 20

Figure 2-9. $\quad$ Test 2 Price Signal (November $\left.19^{\text {th }}, 2003\right)$............................................ 20

Figure 3-1. Network architecture overview of five combined Auto-DR sites ............ 26

Figure 3-2. $\quad$ Albertsons - System Architecture ………….......................................... 32

Figure 3-3. $\quad$ B of A - System Architecture …………................................................ 35

Figure 3-4. $\quad$ GSA Oakland - System Architecture ………...................................... 38

Figure 3-5. $\quad$ Roche - System Architecture ............................................................ 41

Figure 3-6. $\quad$ UCSB - System Architecture .................................................................. 43

Figure 3-7. Example of Whole-Building Method Baseline ……................................. 47

Figure 3-8. Example of Component Method Baseline - Roche ……......................... 49

Figure 4-1. Albertsons - Whole Building Power and Baseline (Whole-Building

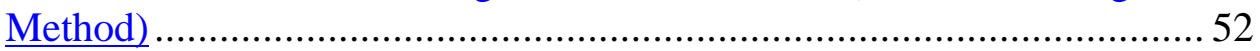

Figure 4-2. Albertsons - Whole Building Power and Savings (Component Method) 53

Figure 4-3. Albertsons - Overhead Lights and Anti-Sweat Door Heaters .................. 54

Figure 4-4. B of A - Whole Building Power and Baseline

(Whole-Building Method) .....................................................................5 55

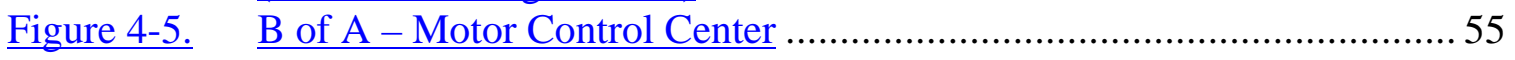

Figure 4-6. $\quad$ B of A - WebGen Chart............................................................................ 56

Figure 4-7. GSA - Whole Building Power and Baseline (Whole-Building Method) . 57

Figure 4-8. $\quad$ GSA - HVAC Demand and Savings (Component Method).....................5 58

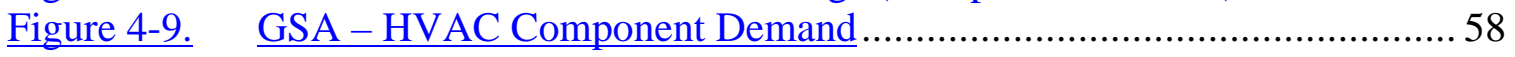

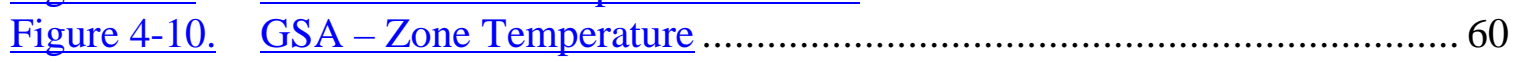

Figure 4-11. Roche-Combined Whole Building Power and Baseline (Whole-Building

Method applied to all Three Buildings) ................................................. 61

Figure 4-12. Roche - Whole Building, Fan Power and Savings (Component Method) 62

Figure 4-13. Roche - Building A2, Zone Temperature ............................................ 62

Figure 4-14. $\quad$ Roche - Building $\mathrm{A} 2, \mathrm{CO}_{2}$ Concentration ............................................6 63

Figure 4-15. UCSB - Whole Building Power and Baseline (Whole-Building Method)64

Figure 4-16. UCSB - Whole Building Power, Cooling Energy and Savings (Component Method)........ 


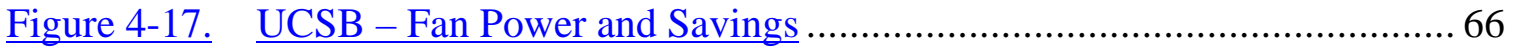

Figure 4-18. UCSB - Average Zone Temperature ……….........................................67

Figure 4-19. Average Shed kW by Price Signal Level (Component Method) ............. 69

Figure 4-20. Average Shed W/ft ${ }^{2}$ by Price Signal Level (Component Method)........... 69

Figure 4-21. Summary of All Sites...................................................................... 70

Figure 4-22. Saving Estimates by Whole-Building Method and Component Method.. 71 


\section{Acknowledgements}

The authors are grateful for the extensive support from numerous individuals who assisted in this project, including: Ron Hofmann, a consultant to the California Energy Commission (CEC), for his conceptualization of this project and ongoing technical support; Laurie ten Hope (CEC); Gaymond Yee (California Institute for Energy and the Environment); George Hernandez; and Karen Herter (Lawrence Berkeley National Laboratory). This project is supported by the Assistant Secretary for Energy Efficiency and Renewable Energy, Office of Building Technology, State and Community Programs of the U.S. Department of Energy under Contract No. DE-AC03-76SF00098. This project could not have been completed without the extensive assistance from the following building owners, managers, and technology developers:

\begin{tabular}{|l|l|}
\hline Albertsons & Glenn Barrett, Mike Water, Ed Lepacek \\
\hline Chevron Energy Services & Bruce Dickinson \\
\hline Engage/eLutions & $\begin{array}{l}\text { Paul Sheppard, Tom Pappas, John Kuhl, } \\
\text { Jagdish Dudhat }\end{array}$ \\
\hline Infotility & Joe Desmond and Nicolas Kardas \\
\hline Itron & Dale Fong \\
\hline Jones Lang LaSalle & $\begin{array}{l}\text { Don Rudy, John Schinter, Steven Press, } \\
\text { William Young, Kent Anderson }\end{array}$ \\
\hline Roche Palo Alto & Jerry Meek, Jeff Stamp \\
\hline $\begin{array}{l}\text { United States General Services } \\
\text { Administration }\end{array}$ & $\begin{array}{l}\text { Edgar Gray, John Goins, Stephen May, } \\
\text { Mark Levi, Jan Wright }\end{array}$ \\
\hline United States Postal Service & Ray Levinson and John Samuelson \\
\hline University of California, Santa Barbara & Jim Dewey \\
\hline WebGen Systems, Inc. & Dirk Mahling, Bob Landry, Glenn Miller \\
\hline West Valley Electric, Aztec & Cory Timms, Dean Cromwell \\
\hline Yamas Controls, Inc. & Janey Kaster, Ken Mason, Jason Doll \\
\hline
\end{tabular}

\section{DISCLAIMER}

This report was prepared as a result of work sponsored by the California Energy Commission (Commission). It does not necessarily represent the views of the Commission, its employees, or the State of California. The Commission, the State of California, its employees, contractors, and subcontractors make no warranty, express or implied, and assume no legal liability for the information in this report, nor does any party represent that the use of this information will not infringe upon privately owned rights. This report has not been approved or disapproved by the Commission nor has the Commission passed upon the accuracy or adequacy of the information in this report. 



\section{EXECUTIVE SUMMARY}

\section{Introduction and Goals}

This report describes the results of a research project to develop and evaluate the performance of new Automated Demand Response (Auto-DR) hardware and software technology in large facilities. Demand Response (DR) is a set of activities to reduce or shift electricity use to improve electric grid reliability, manage electricity costs, and ensure that customers receive signals that encourage load reduction during times when the electric grid is near its capacity. The two main drivers for widespread demand responsiveness are the prevention of future electricity crises and the reduction of electricity prices. Additional goals for price responsiveness include equity through cost of service pricing, and customer control of electricity usage and bills. The technology developed and evaluated in this report could be used to support numerous forms of DR programs and tariffs.

For the purpose of this report, we have defined three levels of Demand Response automation. Manual Demand Response involves manually turning off lights or equipment; this can be a labor-intensive approach. Semi-Automated Response involves the use of building energy management control systems for load shedding, where a preprogrammed load shedding strategy is initiated by facilities staff. Fully-Automated Demand Response is initiated at a building or facility through receipt of an external communications signal - facility staff set up a pre-programmed load shedding strategy which is automatically initiated by the system without the need for human intervention. We have defined this approach to be Auto-DR. An important concept in Auto-DR is that a facility manager is able to "opt out" or "override" an individual DR event if it occurs at a time when the reduction in end-use services is not desirable.

This project sought to improve the feasibility and nature of Auto-DR strategies in large facilities. The research focused on technology development, testing, characterization, and evaluation relating to Auto-DR. This evaluation also included the related decisionmaking perspectives of the facility owners and managers. Another goal of this project was to develop and test a real-time signal for automated demand response that provided a common communication infrastructure for diverse facilities. The six facilities recruited for this project were selected from the facilities that received CEC funds for new DR technology during California's 2000-2001 electricity crises (AB970 and SB-5X).

\section{Automated-DR Test Concept}

A significant goal of this research was to perform a two-week test of fully automated DR test at four to six facilities. We worked with each facility's staff to develop a demand response strategy that would result in a larger electric load shed at higher electricity prices. The test consisted of providing a single fictitious continuous electric price signal to each facility. The technology used for the communications is known as Extensible Markup Language (XML) with "Web services." Control and communications systems at each site were programmed to check the latest electricity price published by the price 
server and automatically act upon that signal. All of the facilities had Energy Information Systems (EIS) and Energy Management and Control Systems (EMCS) that were programmed to automatically begin shedding demand when the price rose from $\$ 0.10 / \mathrm{kWh}$ to $\$ 0.30 / \mathrm{kWh}$. The second level price signal increased to $\$ 0.75 / \mathrm{kWh}$. Five sites participated in the test. The test kept the fictitious prices elevated for three hours. Figure E-1 shows the price signal sent on Wednesday, November $19^{\text {th }}, 2003$.

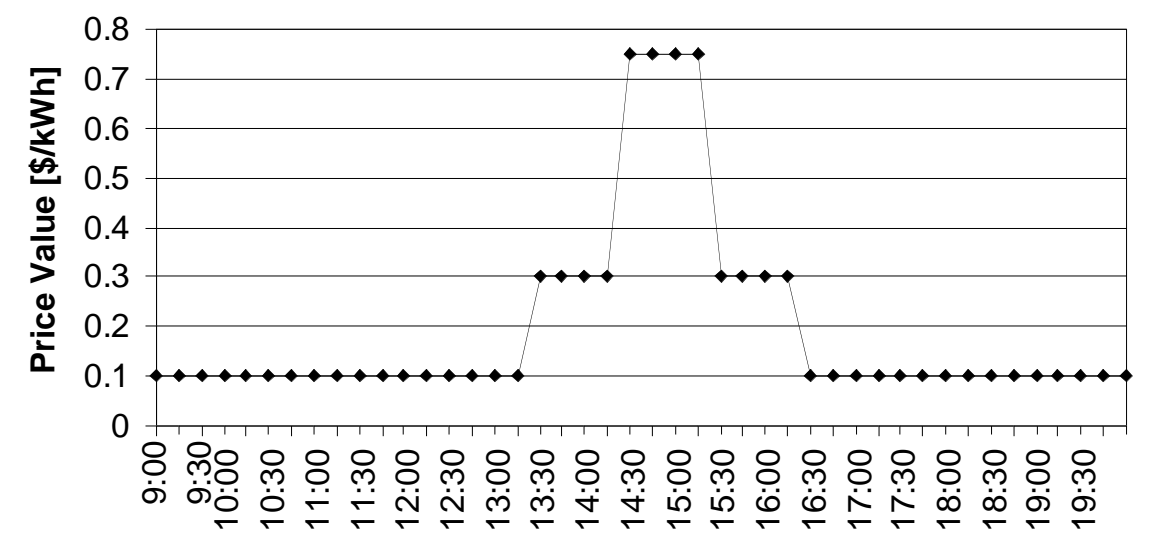

Figure E-1. Fictitious Electric Price Signal Sent to Five Facilities

The sites in the test, listed below with their control and communication systems, represent a diverse set of facilities, control and communications systems, utilities, ownership types, and DR strategies:

- Supermarket - Albertsons, Engage Networks/eLutions - Active Energy Management ${ }^{\mathrm{TM}}$

- Office - Bank Of America, WebGen - Intelligent Use of Energy ${ }^{\mathrm{TM}}$

- Office - General Services Administration, BACnet Reader and BACnet controller

- Office and Cafeteria - Roche Pharmaceuticals, Tridium - Vykon ${ }^{\mathrm{TM}}$

- Library - UC Santa Barbara, Itron - Enterprise Energy Management Suite ${ }^{\mathrm{TM}}$

Figure E-2 shows the geographic distribution of the test sites, the location of the price server and clients, and the location of the development sites from the different system developers. LBNL developed detailed description of the controls and communications infrastructure at each site. In a related effort and to prepare for the test, LBNL developed measurement plans at each site to measure both the whole-facility electric load shed, and component electric load shed. The controls and communications systems developed for the test were also used to measure the sheds. A series of methods to evaluate the sheds were developed based on each site's energy and performance measurements. Non-energy changes in building services were also evaluated at three sites. Extensive interviews of the test participants were conducted to evaluate any disruptions that took place as a result of the test, to understand the technical requirements for the technology implementation, and to gather information on their perspective on DR. 


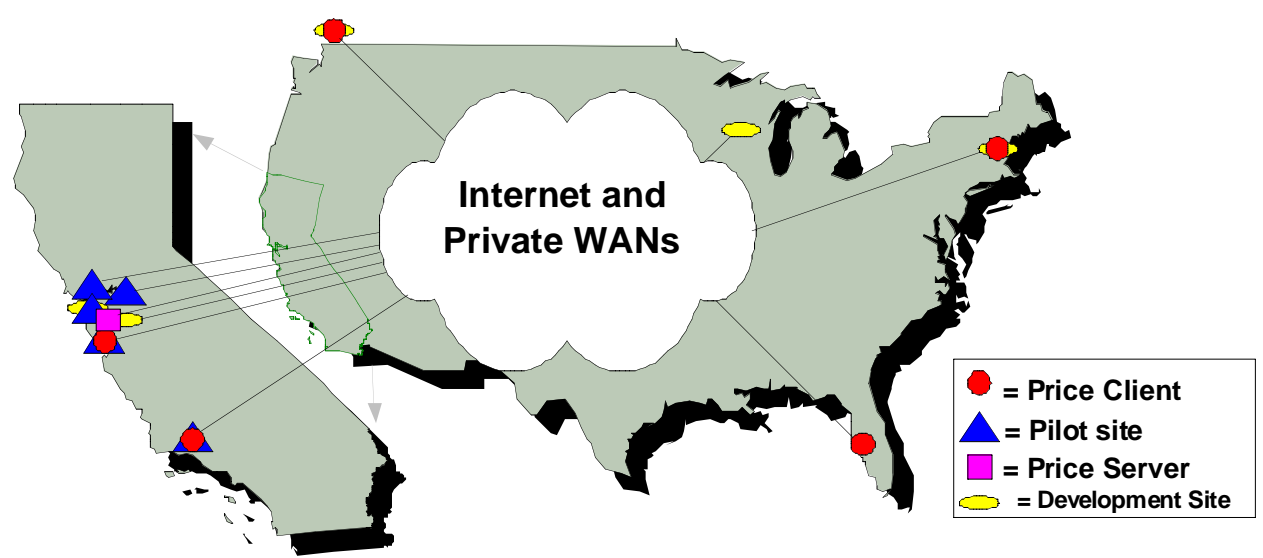

Figure E-2. Geographical Location of Auto-DR Infrastructure

\section{Test Results}

The two-week test period began on Monday, November 10, 2003. LBNL sent the first fictitious high price signal on Wednesday, November $12^{\text {th }}$. Three of the sites had technical problems during the first test, so minimal analysis was conducted on the first test's measured data. The second test on Wednesday, November $19^{\text {th }}$, was successful, with all five buildings simultaneously reducing their electric demand (See Figure E-3). The shed strategies consisted of the following type of control changes: zone set-point change, direct control of fans, resetting duct static pressure, resetting of cooling valves, reduction of overhead lighting, and reduction of anti-sweat heaters.

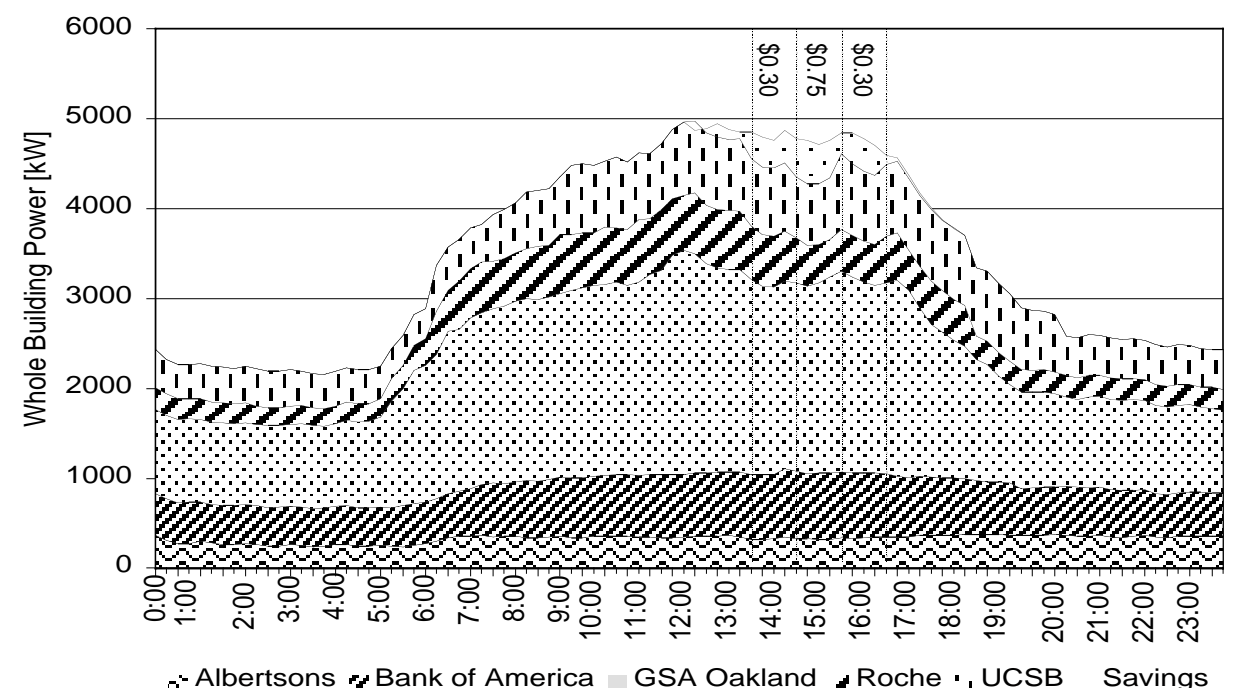

Figure E-3. Auto-DR Electric Load Shed from five sites on Wed. Nov. 19, 2003.

The aggregated total demand for the five facilities was nearly $5000 \mathrm{~kW}$. The maximum peak savings was about $10 \%$ of that load, or about $500 \mathrm{~kW}$. The maximum load reduction at each site ranged from $8 \mathrm{~kW}$ (Bank of America) to $240 \mathrm{~kW}$ (GSA). Area normalized maximum savings ranged from $0.04 \mathrm{~W} / \mathrm{ft}^{2}$ (Bank of America) to $0.83 \mathrm{~W} / \mathrm{ft}^{2}$ (Albertsons). Hourly average electric load reductions for each of the five sites are shown 
in Figure E-4. The maximum savings occurred during the high-price time for three of the five sites. There were no tenant or other complaints at any of the sites, but this was not surprising given that the sheds were not aggressive.

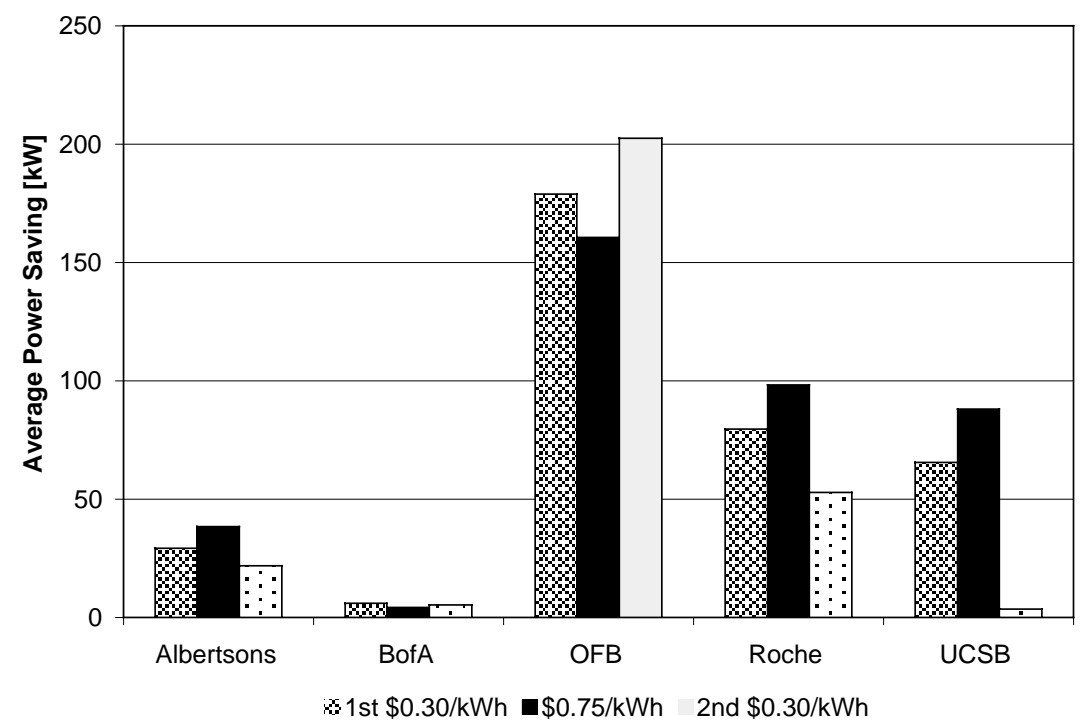

Figure E-4. Auto-DR Electric Load Shed from five sites on Wed. Nov. 19, 2003.

\section{Overall Lessons}

This study has demonstrated a number of key issues that relate to Automated DR, and DR in general:

- Fully automated DR is technically feasible with minor enhancements to current state-of-the-art technology - The facilities that participated in the study used their existing EMCS and EIS systems for the Auto-DR test. In two cases, an electronic interface component was added to provide communications functions necessary for Auto-DR. No additional hardware was required at the other three sites. All five sites required custom software programming to enable Auto-DR functionality. The time required for programming at each site varied from a couple of days to about one month of labor. The technology used offers a glimpse of the issues that may need to be addressed with a large-scale deployment effort. While the Auto-DR infrastructure and associated components performed as intended in this test, additional technical issues associated with a full-scale AutoDR deployment are expected to be significant (see Section 6 - Future Research Topics).

- New Internet technology enhances the capabilities of existing building systems to enable demand response - Although each of the participating facilities had different types of EMCS and EIS systems, they were "unified" in the sense that they all monitored and responded to a price signal from one common price server. The custom software at each site was programmed using the 
emerging technology standards "XML" and "Web services." An examination of the use of XML/Web services and the associated interfaces to existing EMCS and EIS systems is included in this report.

- Automation enhances demand response programs - The electric consumers we worked with indicated that automation of DR is likely to foster greater participation in various DR markets by decreasing the time needed to prepare for a DR event. Automation may likely increase the number of times a facility may be willing to shed loads, and perhaps improve the depth of the sheds, and the number of facilities involved in DR.

- Large facilities support the objectives of DR - This project involved extensive discussions and interactions with five large organizations and institutions. Overall we obtained excellent support and assistance in this research. The energy managers at these organizations believe that DR programs and tariffs will increase in their importance and prominence, and new technology will assist them in participating in these programs.

- New knowledge is needed to procure and operate technology and strategies for DR - DR is a complex concept. Facility operators need to understand DR economics, controls, communications, energy measurement techniques, and the relation between changes in operation and electric demand. Such understanding may involve numerous people at large facilities. Current levels of outsourcing of control services complicate understanding of control strategies and system capabilities.

Outstanding issues that the project was not able to address include how large the load shed would be in warmer weather and an evaluation of the implementation costs for the Auto-DR systems in the current and mature technology markets. Future work will consider these and other issues, including how to scale up to the Auto-DR technologies to include larger numbers of buildings in future tests, and which load shedding strategies are optimal for different building types and climates. 



\section{Introduction}

\section{1-1 Background and Report Overview}

This report describes the results of a research project to evaluate the technological performance of Automated Demand Response hardware and software systems in large facilities. The systems evaluated in this study were installed following California's electricity crisis, which resulted in rolling blackouts and unprecedented high prices. The basic premise of this research project was to conduct a test using a fictitious electricity price sent over the Internet to trigger a demand-response event at the facilities participating in the test. Demand Response can be defined as (1) load response managed by others for reliability purposes, (2) load response managed by others for procurement cost minimization purposes (e.g., load bidding), and (3) price response managed by enduse customers for bill management. ${ }^{l}$ The technology evaluated in this report could be used to support any of these three forms of Demand Response.

The two main drivers for widespread demand responsiveness are the prevention of future electricity crises and the reduction of electricity prices. Additional goals for price responsiveness include equity, through cost of service pricing, and customer control of electricity usage and bills. Demand response has been identified as an important element of the State of California's Energy Action Plan, which was developed by the California Energy Commission (CEC), California Public Utilities Commission (CPUC), and Consumer Power and Conservation Financing Authority (CPA). The CEC's 2003 Integrated Energy Policy Report (CEC, 2003) also advocates DR (Docket No. 02-IEP-1).

For the purpose of this project, we use the term Automated Demand Response or AutoDR to refer to Fully-Automated Demand Response. Levels of automation in demand response may be categorized into manual, semi-automated, and fully automated methods. Manual DR consists of people initiating changes in electric loads by turning off loads, or switching and changing control settings when they receive communications from an external source (such as a phone call, pager signal, or email). Semi-Automated DR consists of a person initiating a pre-programmed load shedding strategy when they receive price signal communications from an external source. Fully Automated DR refers to the use of control and communications technology that listens to an external signal, and then initiates a pre-programmed shed strategy without human intervention.

We use the terms load shedding and demand shedding interchangeably. Load shedding consists of reducing or shedding loads as a DR strategy. One examples of load shedding is to change thermostat settings to reduce cooling electricity demand. All of the demand response techniques evaluated in this report were load shedding as opposed to load shifting. Load shifting consists of modifying the timing of a load, such as a production schedule, or thermal storage using active or passive systems that pre-cool building mass, water, or ice.

\footnotetext{
${ }^{1}$ The CA ISO refers to load response and price response as "dispatched load" and "demand elasticity."
} 
Report Structure. This section (Section 1) describes the project goals and objectives, and discusses previous research. Section 2 describes the project Methodology. Section 3, Systems Characterization, Measurement Systems and Techniques, provides detailed technical descriptions of the controls and communications systems, DR shedding strategies, and measurement systems used in the evaluation of each site. Section 4, Results, reports on the performance of the DR technology and provides extensive discussion of the measurement of the demand-shed savings. Section 5 provides a Discussion of organizational and technological challenges related to DR. Section 6, Summary and Future Directions summarizes a number of key findings and describes outstanding issues and research questions. An extensive set of appendices provides additional documentation of the project background, methods and results. Appendix I provides a review of acronyms and terminology. ${ }^{2}$

\section{1-2 Project Goals}

This report reviews the results of a Fully Automated Demand Response Test. The primary goal of this research project was to evaluate the technological performance of Automated Demand Response hardware and software systems in commercial buildings. There were a number of secondary goals. These included:

- Improving the understanding of Automated DR systems by classifying and characterizing various attributes of the systems available today. We sought to understand the effort required to enable fully automated State-Of-the-Art communication and control technology in large facilities.

- Evaluating the size of demand shedding capabilities in large facilities. Large facilities were chosen for several reasons. First, air conditioning in "commercial buildings" is responsible for about $15 \%$ of the peak electric demand on peak California days (Koomey and Brown, 2002). Automating demand response in large facilities could allow Californians large reductions in peak MW load. Second, the California Energy Commission funded several programs to deliver advanced automation for Demand Responsive heating, ventilation and air conditioning $(\boldsymbol{H V A C})$ systems (Nexant, 2002; KEMA-Xenergy, 2002). This research project sought to explore and build on the capabilities and DR features of new technology deployed as a result of that program.

- Identify technology gaps and prioritize research for future DR systems. Such research is important in helping to identify the key features of how DR functions in practice at the customer level. Similarly, while technology features need to be carefully characterized and evaluated, we also sought to evaluate the market for DR technology, especially the decision-making perspectives of the facility owners and management.

\footnotetext{
${ }^{2}$ The first occurrence of each acronym or terminology is highlighted in bold italics.
} 
- Develop and test a real-time signal for automated demand response. To ensure that the response was fully automated, we sought to develop a signal that initiated the DR strategies soon after it was received. Most of today's real-time-pricing" (or RTP) is actually day-ahead pricing that typically consists of a set of 24 hourly prices. We did not want to give the facilities staff time to manually respond in our test. Instead, we used a 15-minute ahead RTP signal, as further described in Section 2.

\section{1-3 Previous Research}

The California Energy Commission (CEC) and New York State Energy Research and Development Agency (NYSERDA) have been the leaders in demonstrating demand response programs utilizing enabling technologies. Several studies have investigated the effectiveness of the demand responsive technologies implemented in the California and New York efforts.

Nexant has documented its evaluation of the CEC's Peak Load Reduction Program in California in a series of reports (Nexant 2001 and Nexant 2002). These reports document the performance of all the funded technology projects including the magnitude of the response and the cost associated with it.

During the same period, LBNL and Pacific Northwest National Laboratory (PNNL) studied the effectiveness of enabling technologies on customer performance in priceresponsive load programs in California and New York (Goldman et al, 2002). The data for this study came from a survey of 56 customers working with five contractors participating in the CEC and NYSERDA-sponsored DR programs. For New York, these data were combined with actual load curtailment data to quantify effectiveness.

LBNL and PNNL worked with Neenan Associates to evaluate the effectiveness of New York Independent System Operator (NYISO) Demand Response Programs during the summer of 2002. A detailed survey was administered to evaluate the technology used by the program participants. The connection between the actual performance under the NYISO programs and the technologies was characterized (Neenan et al, 2003). As part of this project Kintner-Meyer et al (2003) summarized the methodologies that can be used to evaluate a facility's demand responsive capabilities and response technologies.

At the same time as the Auto-DR research project documented in this report, CEC funded another research project that investigated the customer response to day-ahead wholesale market electricity prices in New York. LBNL and Neenan Associates worked with Niagara Mohawk Power Company to combine measured interval data for large customers with the results of a survey that, among other things, collected data related to the enabling technology endowment of the same customers. The effect of the technology endowment on responsiveness to real time prices and on customer choices regarding supplier, hedging and NYISO program participation was investigated (Goldman et al, 2004).

Previous research at LBNL and elsewhere has examined methods to measure demandshed savings. The California Independent System Operator's (ISO) 2001 Demand Relief 
program used the average power from the 10 previous days as a baseline against which to measure the magnitude of demand shed. This has been a reasonable method for industrial demand shedding, which is dominated by process load changes. This approach does not transfer to the building sector, as it fails to account for the correlation between building electricity use and weather. Demand response days are often warm days, and the baseline load shape for a warm day may be greater than the 10 previous days. LBNL and others have conducted research into evaluation methods. The research project documented in this report included a significant effort related to developing and evaluating new and existing methodologies (See Section 3 and Appendix II for descriptions and further details).

In addition to research concerning utility programs, controls, and communications systems, several research studies have examined various topics concerning DR in commercial buildings such as how to operate buildings to maximize the demand response and minimize the loss of services from DR. Kinney et al reported on weather sensitivity of peak load shedding and power savings from setting up temperatures in buildings to reduce cooling loads (Kinney et al, 2001). Demand limiting and other peak demand control strategies are not new. Previous work from Piette described possibilities for using the control systems to reduce and control peak electrical demands (Piette, 1991). A recently developed guide on Enhanced Automation (KEMA-Xenergy, 2002) was developed for the California Energy Commission to help commercial building owners evaluate control system enhancements and peak reduction strategies .

\section{EIS, EMCS and DR - Change and Overlap}

The building controls industry, like other industries, is undergoing a series of dramatic changes, resulting in the development of new features that take advantage of advanced computing and communications systems. Recent research by LBNL and others has evaluated the capabilities, features, and cost-effectiveness of new technologies for building energy efficiency and demand response (Motegi et al, 2003a and 2003b). Energy Information Systems (EIS) have evolved out of the electric utility industry in order to manage time-series electric consumption data. Since EIS products are relatively new technologies, they are changing quickly as the market unfolds. EIS products are being developed to include a wide variety of features and a fair level of complexity to satisfy the wide range of client needs. Other energy management technologies have also expanded their functionalities.

Figure 1-1 shows the overlap and relationships between EIS, energy management and control systems (EMCS) and demand response systems (DRS). The technologies in these domains differ, yet there are a number of overlaps in functionality and purpose. The EMCS provides energy management and control using both Web-based and non-Webbased systems. The demand response field has developed systems that enable utilityoperated demand response programs or other demand curtailment measures (e.g., responsive thermostat ${ }^{3}$, direct load control devices $\left.{ }^{4}\right)$. Such systems may not be connected

\footnotetext{
${ }^{3}$ A thermostat that can receive external signals and respond by adjusting temperature settings.
} 
with the electric meter (like that of an EIS) or a centralized control (like that of a WebEMCS). This project is concerned with the area of intersection among these domains. This intersection involves remote control of demand response, electric metering, and integrated facility control. The technologies involved in this study have capabilities in each of these three areas, but are all based on different system architectures and linkages with existing legacy control systems.

\section{Types of Web-Based Energy Information Systems (EIS)}

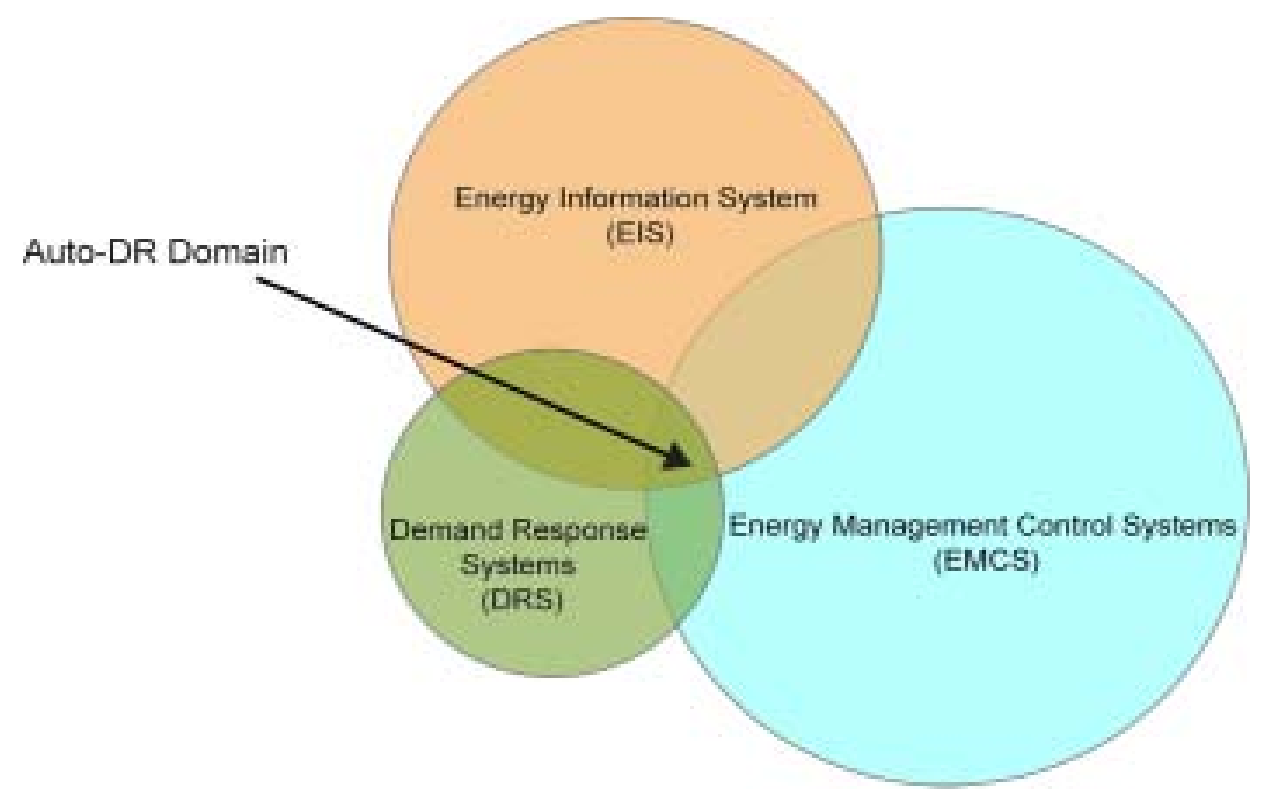

Figure 1-1. Overlap Between Energy Information Systems (EIS), Energy Management Control Systems (EMCS) and Demand Response Systems (DRS)

\footnotetext{
${ }^{4}$ Devices that can interrupt power supply to individual appliances or equipment on consumer premises by the utility system operator or ISO.
} 


\section{Methodology}

This section provides an overview of the project methodology, including:

- Project overview and timeline

- Site selection criteria and sites considered

- Site characteristics and background

- Auto-DR system description

- Controls and communications upgrade description

- Electric price signal and test description

- Assessment of Lead Users of Auto-DR

\section{2-1 Project Overview and Timeline}

The Automated DR project took place over approximately two years, beginning with a planning activity in summer 2002. The 11 major milestones are shown in Table 2-1.

Table 2-1. Project Timeline and Milestones

\begin{tabular}{|l|l|}
\hline Milestone & Time Period \\
\hline Project Planning & Summer - Fall, 2002 \\
\hline Interviews with Nexant & January 2003 \\
\hline Owners Information Developed & February 2003 \\
\hline Participant Conference Call & August 2003 \\
\hline Collection of MOUs & September 2003 \\
\hline Site Visits, Monitoring Install Completed & October 2003 \\
\hline Final Trouble shooting & November 12,2003 \\
\hline Test Period & November $10-24,2003$ \\
\hline Final Successful Test & November 19,2003 \\
\hline Data Analysis & November 2003 - February 2004 \\
\hline Final Report & March 2004 \\
\hline
\end{tabular}

The project was conceptualized by Ron Hofmann, a consultant to the California Energy Commission who expressed an interest in evaluating the automation capabilities of control and communications systems in large facilities. LBNL began planning the project in the early summer of 2002, developing a detailed project description, recruiting a research team, and conducting initial discussions with potential project participants about the feasibility of a fully automated DR test. LBNL met with KEMA-Xenergy in summer 2002 to review the test concept's feasibility as KEMA-Xenergy had recently completed case studies of control systems and DR and two guidebooks on Enhanced Automation (www.consumerenergycenter.org/enhancedautomation).

LBNL met with analysts from Nexant in January 2003 to review Nexant's experience in the evaluation of the sites that participated in AB970 and SB-5X. The meeting included a discussion of various topics, such as which DR participants used the most advanced technologies, what levels of automation existed among the sites, and the current status of the technologies at the sites. Since there were few DR programs in 2002, many of the 
systems installed as part of these programs were taken out of service because of the lack of financial incentives to maintain them.

A detailed project description, including details of the proposed Auto-DR test, designed to inform building owners about the project was developed in February 2003 (see Appendix III). This document was used in conversations with building owners, property managers, control system and EIS vendors, and others interested in the Auto-DR test. From April through July, 2003, LBNL conducted extensive discussions with potential project participants concerning their interests and motivation in participating in the Automated DR test. Several site visits were conducted. LBNL also worked with Infotility to define the technology requirements for the test.

A complete set of project information was developed and communicated to the participants in a teleconference that took place in August 2003, as further described in Section 2. Five MOUs (memorandums of understanding) were collected by September 2003. One site that participated in the test did not complete the MOU, but this was not a requirement of the test. Rather, the MOU served as a communication vehicle to define the terms of the test and LBNL's expectations of the participating sites. Final site visits and monitoring plans were completed in October 2004. The monitoring plans reflected LBNL's understanding of the electric demand shedding strategies defined by each site and outlined what measurements were to be taken during the shed to quantify the shed savings.

The test period was delayed until mid November. It would have been more ideal to conduct the test during more typical summer weather, but that was not possible due to the additional time required to fully program and debug the Automated DR software. The final test took place during the November $10^{\text {th }}$ to $24^{\text {th }}, 2003$ time frame. The initial high price period took place on November $12^{\text {th }}, 2003$. Several of the sites did not respond as intended on the first test. During the $2^{\text {nd }}$ test on November $19^{\text {th }}, 2003$, all five sites successfully executed their demand shedding strategies as planned. The test was followed by extensive data collection and analysis activities, as described below. A set of interviews with each of the building owners was conducted to evaluate their experience during the test.

\section{2-2 Selection Criteria and Sites Considered}

One objective of this project was to evaluate the technological performance of different controls and communication systems related to participation of demand and end-use systems in dynamic price programs. Thus, we tried to select sites that represented a diverse set of facilities utilizing different technologies, based on the following characteristics:

- Facilities (different types of commercial and light industrial)

- Energy Information Systems (EIS)

- Energy Management Control Systems (EMCS)

- Gateways

- Utilities serving the facilities 
- Ownership type (government, private sector, chains, etc.)

- End-Use Load Shedding Strategies

Additionally, we looked for demonstrated DR capability and a willingness to share information on facility operation, facility characteristics and monitored data for the time periods before and during the Auto-DR tests. All of the sites selected had received CEC funds that were available following the electricity crisis to install advanced technologies (See Appendix IV). As part of that program, a CEC evaluation contractor (Nexant 2002) documented that the selected sites demonstrated some capability to shed demand.

Our approach to identifying candidates for the Auto-DR tests was varied. We used several leads, including the list of customers that CEC had funded for DR improvements. In some cases we approached the owners and operators directly-especially in cases where we had worked with them on other research projects (UC Santa Barbara and GSA). In other cases, we approached EIS vendors and identified potential buildings, then approached the owner or operator. As a result of our initial test-site identification effort, we came up with the following list:

- University of California (UC) Santa Barbara and other UCs

- Long Beach State and other California State Universities

- United States Postal Service

- Calif. Dept of General Services

- General Services Administration

- Albertsons

- Roche Pharmaceuticals

- Bank of America

- Shopping Malls (Infotility customers)

\section{2-3 Site Characteristics and Background}

Based on our meetings with facility owners and operators, EIS vendors and Nexant we developed a list of six facilities meeting the criteria for the Auto-DR test. Final selection of the following five sites was based on the interest and commitment from discussions with owners and vendors, and included: ${ }^{5}$

- Albertsons, Engage Networks/eLutions (Supermarket)

- Bank Of America, WebGen Intelligent Use of Energy (Office)

- General Services Administration, BACnet Reader and BACnet controller (Office)

- Roche Pharmaceuticals, Tridium Vykon (Office and Cafeteria)

- UC Santa Barbara, Itron enterprise Energy Management Suite (Library)

Prior to the upgrades required for the Auto-DR test, the controls and communications systems used in the participating buildings were substantially similar to other large commercial buildings in California. All of the sites had microprocessor based Energy Management and Control Systems (EMCS) that are typical for large commercial

\footnotetext{
${ }^{5}$ USPS - San Jose - CMS Viron/Chevron (Distribution Center) dropped out of consideration when Chevron bought Viron and there were information technology upgrade problems
} 
buildings of this type. A variety of EMCS brands were present. The systems were between five and fifteen years old. Some of the sites had heterogeneous systems of various ages, brands and product lines within a given facility.

However, the facilities selected for the Auto-DR test differed from most commercial buildings in California because each site had the capability to remotely monitor and control HVAC or lighting equipment over the Internet. Although these remote control and monitoring features, known collectively as telemetry, are becoming increasingly popular in newly installed EMCSs, they are still uncommon within the installed base of commercial buildings in California. For this reason, the 2003 Auto-DR participating sites were a select group. The demonstrated DR capability of these facilities is summarized in Appendix IV.

In August 2003, a meeting was arranged where all the owners, operators and EIS vendors were invited to participate. The objective of this meeting was to provide the participants with an overview of the project, allow them to hear about related technology at the other sites and answer any of their questions. Following the meeting, an information package was sent to all the participants (Appendix III). This package included: (1) a memorandum of understanding for facility owners to review and sign, (2) a detailed description of the price signal, (3) a questionnaire to collect site specific information relating to the demand response actions that the site could implement, (4) another questionnaire to collect technical information about the communication hardware and capabilities at each site, and (5) a tentative timeline for the different stages of the project. During the following weeks, the project was delayed by the time required to finalize the $X M L$ signal specifications and associated software requirement. Once the specifications were circulated (in late August, 2003), numerous conversations with control and EIS vendors were needed to identify company individuals qualified to discuss the technology development and authorize the work with us.

Table 2-2 summarizes the characteristics of each site. The sites differ as to which enduses are included in the whole-building electric meter because three of the sites are part of larger campuses and have central cooling plants. Cooling energy delivered to each building is not measured at two of these. If cooling energy was not discretely measured for a given test building, its effect on energy and demand savings (or loss) was not included in the calculations ${ }^{6}$. The table shows that the November 2003 whole-building power (WBP) peak demand ranged from 401 to $2710 \mathrm{~kW}$, or 2.8 to $8.0 \mathrm{~W} / \mathrm{ft}^{2}$. The summer peak demand for summer 2003 is also shown (2003 WBP Peak).

Table 2-2. Summary of Sites

\begin{tabular}{|l|l|l|l|l|l|}
\hline & Albertsons & Bank of & GSA & Roche & UCSB \\
\hline \hline
\end{tabular}

\footnotetext{
${ }^{6}$ Potentially it may also contribute to negative savings, because reduction of airflow requires lower chilled water temperature to maintain same zone temperature.
} 


\begin{tabular}{|c||c|c|c|c|c|}
\hline & & America & Oakland & & \\
\hline Location & Oakland & Concord & Oakland & Palo Alto & $\begin{array}{c}\text { Santa } \\
\text { Barbara }\end{array}$ \\
\hline Use & Supermarket & $\begin{array}{c}\text { Office } \\
\text { (Retail) }\end{array}$ & Office & $\begin{array}{c}\text { Pharmaceutical } \\
\text { laboratory } \\
\text { (Office/Cafeteria) }\end{array}$ & Library \\
\hline $\begin{array}{c}\text { Cooling } \\
\text { System }\end{array}$ & Included & Not included & Included & Not included & Included \\
\hline Area & 50,000 & 211,000 & $\begin{array}{c}978,000 \\
\text { (Conditioned) }\end{array}$ & $192,000 *$ & 289,000 \\
\hline $\begin{array}{c}\text { 2003 Annual } \\
\text { WBP peak }\end{array}$ & $431 \mathrm{~kW}$ & $\mathrm{~N} / \mathrm{A}$ & $3795 \mathrm{~kW}$ & $782 \mathrm{~kW}$ & $1311 \mathrm{~kW}$ \\
\hline $\begin{array}{c}\text { WBP peak } \\
\text { (Nov) }\end{array}$ & $401 \mathrm{~kW}$ & $999 \mathrm{~kW}$ & $2710 \mathrm{~kW}$ & $706 \mathrm{~kW}$ & $866 \mathrm{~kW}$ \\
\hline $\begin{array}{c}\text { Peak W/ft2 } \\
\text { (Nov) }\end{array}$ & 8.0 & 4.7 & 2.8 & 3.6 & 3.0 \\
\hline
\end{tabular}

* Includes three buildings - Office Building A2 is $101,078 \mathrm{ft}^{2}$, FS is $23,159 \mathrm{ft}^{2}$, SS is $67,862 \mathrm{ft}^{2}$.

\section{Facility Descriptions}

\section{Albertsons}

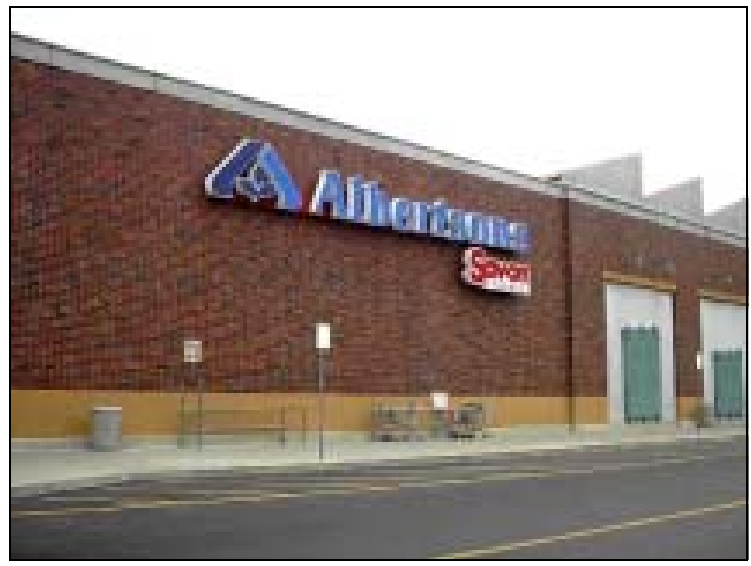

Figure 2-2. Albertsons

- Facility: Supermarket

- Utilities serving the facilities: PG\&E at Oakland Site

- Ownership type: Large owner occupied site with numerous locations in California

- Type of tenants: Customers

The store opens at 6:00 am and closes at midnight, seven days a week. Some components are shed or turned off during nighttime mode operation from midnight to 6:00 am. There is no difference in the operational patterns between weekdays and the weekend. Due to the refrigeration demand for fresh and frozen foods, Albertsons electric demand per square foot $\left(8.0 \mathrm{~kW} / \mathrm{ft}^{2}\right)$ is the highest of all five sites. 


\section{Bank of America}

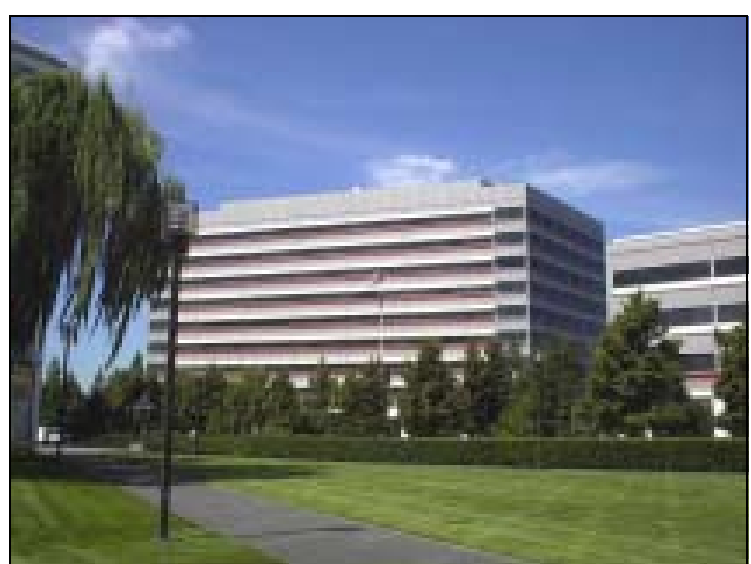

Figure 2-3. Bank of America - Concord Data Center, Building B

- Facility: Large private sector office building

- Utilities serving the facilities: Direct access and PG\&E at Concord Site

- Ownership type: Large owner occupied site with numerous locations in California

- Type of tenants: Company employees

The Bank of America has four buildings (A, B, C and D) at the site. Buildings A, B and $\mathrm{C}$ are office buildings. Building $\mathrm{D}$ has a large data center. The cooling plant is located at Building D and supplies chilled water to all the buildings. The Auto-DR test was demonstrated at Building B only.

\section{Oakland Federal Building (GSA)}

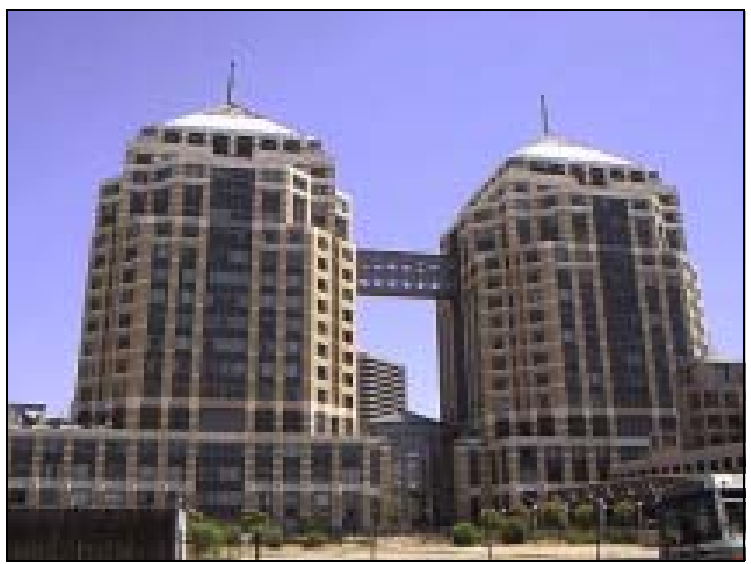

Figure 2-4. GSA Oakland Federal Building

- Facility: Large Government Office

- Utilities serving the facilities: Direct access and PG\&E in Oakland

- Ownership type: Large owner occupied federal building

- Type of tenants: Governmental employees 
The Oakland Federal Building consists of twin towers with a gross floor area of 1.1 million square feet and included $971,000 \mathrm{ft}^{2}$ of office space, a 7,200 $\mathrm{ft}^{2}$ computer center, and a 36,000 $\mathrm{ft}^{2}$ parking garage. There are four main air systems which include cold deck, hot deck and return fans (eleven main air handlers and 47 smaller supply fans). There are variable frequency drives (VFD) on 22 of these fans. The distribution system includes dual-duct and variable air volume (VAV) with reheat serving the perimeter zones, and single-duct VAV (without reheat) serving the core zones. The building achieved the ENERGY STAR Label for Buildings in 2000 without major retrofits or additional commissioning (Piette, et.al. 2002).

\section{Roche Pharmaceuticals}

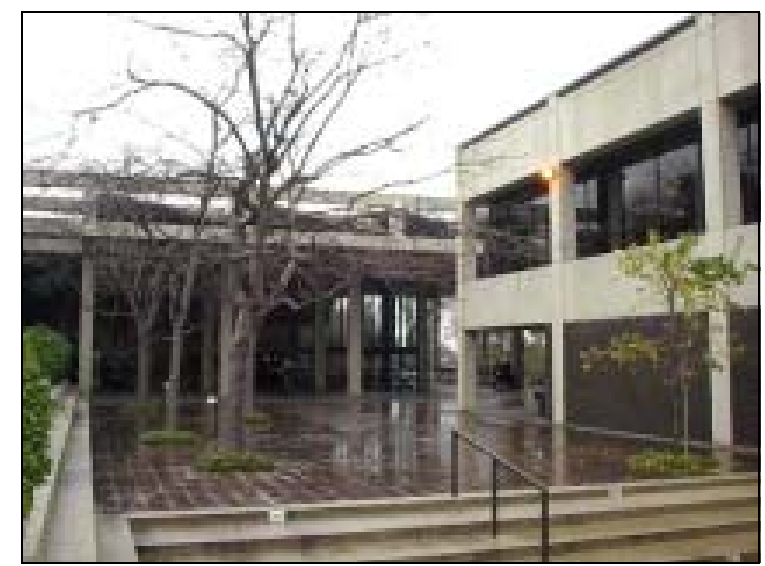

Figure 2-5. Roche Palo Alto - Building FS (left) and A2 (right)

- Facility: Private research campus

- Utilities serving the facilities: City of Palo Alto Utilities

- Ownership type: Large owner occupied research facility

- Type of tenants: Laboratory staff

Roche Palo Alto, situated in the Stanford Research Park, is one of the company's four pharmaceutical research centers in Silicon Valley. The campus consists of seventeen buildings with a total area of 760,000 square feet. These buildings are administrative buildings and pharmaceutical laboratories. The peak load for the campus is $15 \mathrm{MW}$. 


\section{University of California, Santa Barbara}

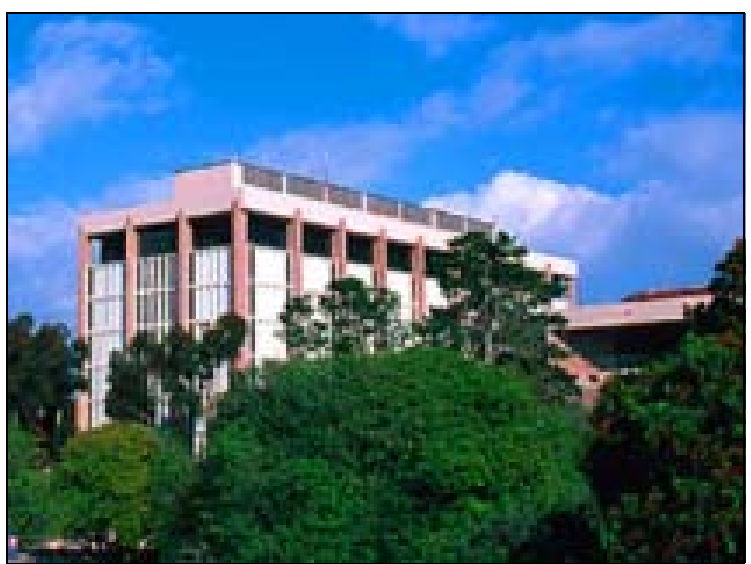

Figure 2-6. UCSB - Davidson Library

- Facility: Campus, although the tests will be in the main library

- Utilities serving the facilities: Direct access (APS) and PG\&E in Santa Barbara

- Ownership type: Large owner occupied campus

- Type of tenants: Students, library staff

University of California, Santa Barbara (UCSB) has a large campus with 4.5 million square feet of building area, holding approximately 22,000 students, faculty, and staff. UCSB campus has a "virtual central plant". ${ }^{7}$ The main library (Davidson Library) was chosen for the Auto-DR test. The library consists of three adjacent buildings, II, III, and IV. The library is connected to the virtual central plant. The library was chosen because it houses a large amount of books, which has a substantial thermal mass effect.

Considering the thermal mass effect, the interior temperature change is slow, and it is easier to maintain the occupants' comfort during the tests. Another reason is because the library is a non-critical space where complaints would be minimal. The library is open from 8:00 am to midnight, from Monday to Thursday. The HVAC equipment starts operation at 6:00 am and stops at 8:00 pm. The library closes earlier at 9:00 pm on Friday, and the equipment stops at 5:00 pm. The library is open on the weekend, but for less hours than seen in the Monday through Thursday schedule. The weekend electrical demand is lower than during the week. Independent of our Auto-DR tests, the energy manager of UCSB occasionally sheds the electric demand to test his energy saving strategies.

\footnotetext{
${ }^{7}$ The chilled water loop that runs through the campus links multiple buildings and allows any single chiller to supply to all the buildings when the demand is low. Most of the medium-large sized campus buildings are connected to the chilled water loop.
} 


\section{2-4 Automated Demand Response System Description}

This section describes the various system components that were used to implement the 2003 Auto-DR test.

\section{System Functionality}

The Automated Demand Response System generated an XML price signal from a single source on the Internet. Each of five disparate commercial building sites monitored the common price signal and automatically shed site-specific electric loads when the price increased beyond pre-determined thresholds. Other than price signal scheduling, which was set up in advance by the project researchers, the system was designed to operate without human intervention during the two-week test period. Figure 2-6 shows the overall sequence of the Auto-DR network communications.

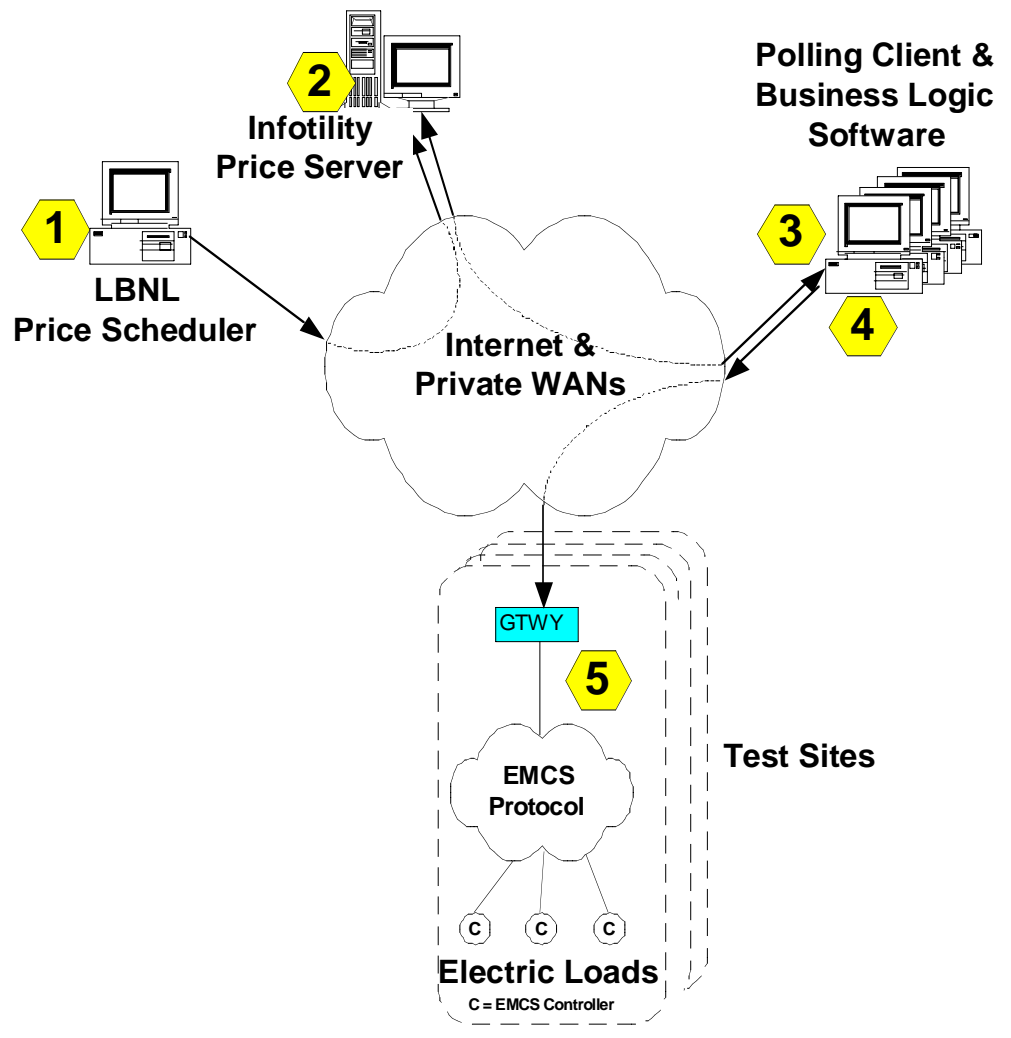

1. LBNL defined the price vs. time schedule and sent it to the price server.

2. The current price was published on the server.

3. Clients requested the latest price from the server every few minutes.

4. Business logic determined actions based on price.

5. EMCS (energy management control system) carried out shed commands based on business logic.

Figure 2-7. Auto-DR Network Communications Sequence 


\section{System Geography}

Although all of the test sites were in California, the Auto-DR infrastructure, polling client software and software developers were distributed throughout North America (Figure 2-7). Existing networks, including the Internet and private WANs were used for these long-distance communications at no additional cost to the participants.

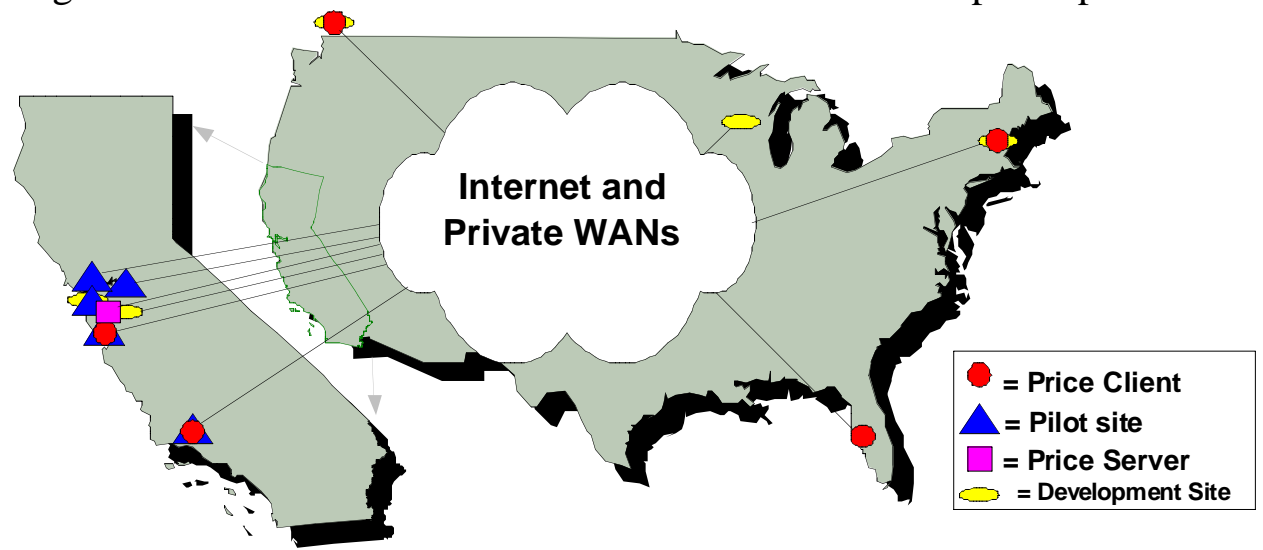

Figure 2-8. Geographical Location of Auto-DR Infrastructure

\section{Web Services / XML}

The infrastructure of the Auto-DR System is based on a set of technologies known as Web services. Web services have emerged as an important new type of application used in creating distributed computing solutions across the Internet. Properly designed Web services are completely independent of computer platform (i.e., Microsoft, Linux, Unix, Mac, etc.). The following analogy helps to describe Web services: Web pages are for people to view information on the Internet; Web services are for computers to share information on the Internet. Since these interactions often occur without human intervention, this technology is sometimes referred to as "Machine-to-Machine" or "M2M". M2M is a superset of technologies that includes some XML/Web services based systems.

XML is a "meta-language" - a language for describing other languages - that allows design of customized markup languages for different types of documents on the Web (Flynn, 2003). It allows designers to create their own customized tags, enabling the definition, transmission, validation, and interpretation of data between applications and between organizations (Webopedia, 2004). Standard communication protocols used on the Internet and $\boldsymbol{L A N} / \boldsymbol{W A N s}$ (local area network/wide area network) including $\boldsymbol{T C P} / \mathbf{I P}$, $\boldsymbol{H T T P}$ and $\boldsymbol{S O A P}$ are used to transfer XML messages across the network.

\section{Price Scheduling Software}

A client software application was used by researchers at LBNL to set-up the price vs. time profile in the price server. The signal was designed so that the price was published 15 minutes ahead of when it was to take effect. The purpose of the 15-minute advanced notice was to give operators enough time to manually "opt out" of the shed if they desired. In the November tests, none of the operators opted out of the sheds. 
The price profile could be set up hours, days or weeks in advance by researchers, however the building operators were not given advanced knowledge of upcoming fictitious price increases (other than the 15 minute ahead price signal). More details about the price signal are provided in Section 2-6, Electric Price Signal and Test Description.

\section{Web Services - Server}

At the heart of the Auto-DR System is the Web services server. The server publishes the current fictitious price for electricity in $\$ / \mathrm{kWh}$. The server was hosted at a co-location site in Fremont, CA. Co-location companies (co-lo) provide computer-hosting services for systems that require "high availability". The co-lo site has high bandwidth Internet access, UPS with backup generators, load balancing, guarded access control and other features necessary to prevent unscheduled system shutdowns.

\section{Web Services - Client and Business Logic Software}

The polling client is the software application that polls (i.e., checks) the Web services server to get the latest price from the aforementioned Web services server. This is known as "pull architecture" because the clients pull information from the server. The polling client software resides on a computer managed by the building operators or their representatives for each site. The client software may reside either on a computer at the building site or remotely. In this test, each client polled the server at a user-defined frequency of once every 1 to 5 minutes. All client interactions with the server were time stamped and logged. All of the clients and the server used Internet based time servers that provide end-to-end accuracy within plus or minus one second.

\section{Real-Time Shedding}

The polling clients for each site received the latest upcoming pricing from the server about 14 minutes before the new pricing actually took effect ( 15 minute ahead pricing minus the polling delay). Rather than waiting until the new price took effect, business logic at all of the test sites shed electric loads immediately upon receipt of an upcoming rise in price. In other words, each site initiated shedding of electric loads about 14 minutes before the price went up. This near real-time shed of electric loads is advisable as prices are rising so as to allow HVAC equipment to ramp down gradually before the new pricing becomes effective. However, this quick and simple approach could be economically detrimental if HVAC and lighting systems start back up 14 minutes too soon, while electric prices are still high. From the small sample of buildings in this test, it appears that the simplicity of real-time load shedding outweighs the benefits of 15 minute delayed action.

\section{Polling Client Price Verification}

The price server included a feature that verified that the clients each received correct pricing information. This feature was implemented by requiring that each client's request for the latest price included its current price and associated time stamp. All pricing data were stored in a database for future analysis.

Although the original intent of this feature was to verify client receipt of server generated pricing, there was another unforeseen benefit as well. When preliminary testing began, 
researchers were able to observe when each site came online by viewing its polling history. Even after all the sites were online, this feature was used as an indicator of system-wide communication status. There were cases where clients would stop polling for known or unknown reasons. Because of the communication status indicator, program managers and system administrators were able to quickly observe these intermittent losses of communication and make phone calls to resolve the problems.

\section{2-5 Controls and Communications Upgrades}

In order to add Auto-DR functionality to each test site, some upgrades and modification to the controls and communications systems were required. The upgrades were built to work in conjunction with the existing EMCS and EIS remote monitoring and control infrastructure in place at each site.

\section{Custom Software - Price Polling Client}

Web services price polling client software was created and installed for each site. Although an example template was provided, it was necessary to customize the client software for use within the software development environment and Information Technology (IT) infrastructure at each organization.

\section{Business Logic}

The purpose of the Business Logic Software is to determine what actions, if any, should be automatically initiated based on a given electricity price. The business logic can be customized for each site as required. In most cases, the business logic used at the test sites switched between three modes of operation (normal, shed level 1 and shed level 2) as prices changed between $\$ 0.10 / \mathrm{kWh}, \$ 0.30 / \mathrm{kWh}$ and $\$ 0.75 / \mathrm{kWh}$ respectively.

Albertsons added additional business logic that forced the systems back into normal mode after 3:30 pm, regardless of price. In the November tests, the business logic software was typically installed on the same computers as the price-polling clients.

\section{Site Specific EMCS modifications}

The EMCSs at each site were used to control electric loads such as HVAC equipment and lighting. Various methods were used to communicate the business logic mode commands to the EMCSs. The most integrated systems ( $\mathrm{B}$ of A, Roche) were able to send and receive control commands directly to EMCS controllers using communication gateways to translate between enterprise networks (i.e., TCP/IP) and control networks (e.g., BACnet, LonTalk).

The other sites used relay contacts to connect the data networks and control networks. In this method, a special IP relay device is controlled remotely over the enterprise network. The onsite EMCS monitors the remotely controlled IP relay contacts and sheds HVAC or lighting loads according to predetermined logic. Physical installation of hardware and wiring was required at sites where IP relay devices were used (Albertsons, GSA and UCSB). It was necessary to install 150 feet of low voltage wiring between the existing IP relay and the EMCS controller at the Albertsons site. 


\section{2-6 Electric Price Signal and Test Description}

The different programs and tariffs for DR use different types of signals to motivate response. Day-ahead real-time-price tariffs, for example, use a 24-hour price matrix for the following day that is communicated to the customer by the late afternoon. In an hourahead real time price tariffs program, customers could be notified of upcoming changes in prices one hour ahead of time. In other emergency- or reliability-based programs, curtailment signals may be sent out by multiple modes such as pager, phone, or fax. These curtailment signals are usually not price signals, but binary signals that indicate when it is time to curtail. The lead time for such signals may vary from 2 hours to minutes depending on the type of the program. The test signal schema for the 2003 test was designed to allow customization so as to accommodate a wide range of tariff and program types.

As mentioned above, the polling clients published the price signal used in the 2003 test on a Web services interface and was available for viewing at any time. The published price changed periodically based on the aforementioned price scheduling software. The shape of the price vs. time profile was designed by LBNL researchers based on the following reasoning. The signal profile needed to meet our research requirements (e.g., price spikes were programmed to occur during the hottest time in the afternoon) and also minimize potential negative effects to the building operators. The signal was designed to provide some predictability to the operators in terms of the frequency of the changes, the level of the prices and the duration of the changes.

In order to meet these criteria the shape of a signal was designed to conform to the following rules. These rules were shared with the building operators in advance of the tests.

- When the price level changed, the level stayed the same for at least one full hour.

- There were three levels of price: normal $(\$ 0.10 / \mathrm{kWh})$, medium $(\$ 0.30 / \mathrm{kWh})$ and high $(\$ 0.75 / \mathrm{kWh})$.

- The duration of price changes to higher than normal would not exceed 3 hours (thus shorter than critical peak pricing (CPP)) and prices would move above normal only once during one day (once prices moved, the facility could be sure that it would be back to normal within 3 hours and would not move again for the day).

- Changes to the price signal were confined to a specific time window: 12:00 pm to 7:00 pm (weekdays).

- The tests for all sites would take place within a two-week period. A maximum of two tests would occur during this time. All five sites were tested simultaneously.

The actual signal sent on November $12^{\text {th }}, 2003$ is presented in Figure 2-8. This figure was downloaded from the user interface of the price scheduling application. The intention of the first test was to keep the signal as simple as possible but still test two levels of response. 


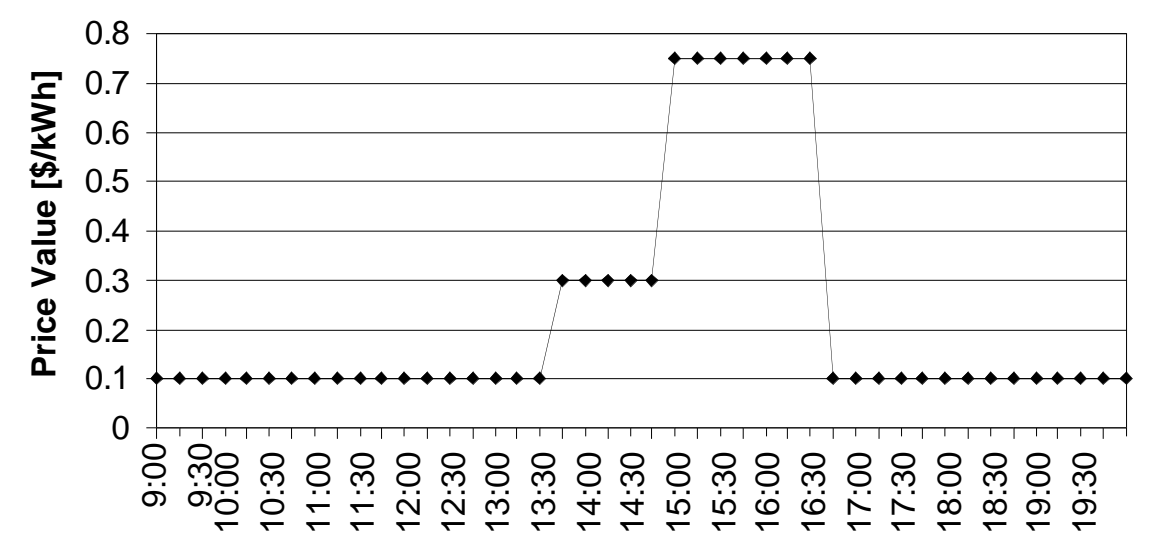

Figure 2-9. Test 1 Price Signal (November $12^{\text {th }}, 2003$ )

The price signal during the second test (November $19^{\text {th }}, 2003$ ) was more complex. As shown in Figure 2-9, the signal was programmed to change from normal pricing to medium, then to high, then back to medium, and finally back to normal. The purpose of this pattern was to identify problems that might occur when the systems came out of a curtailment event.

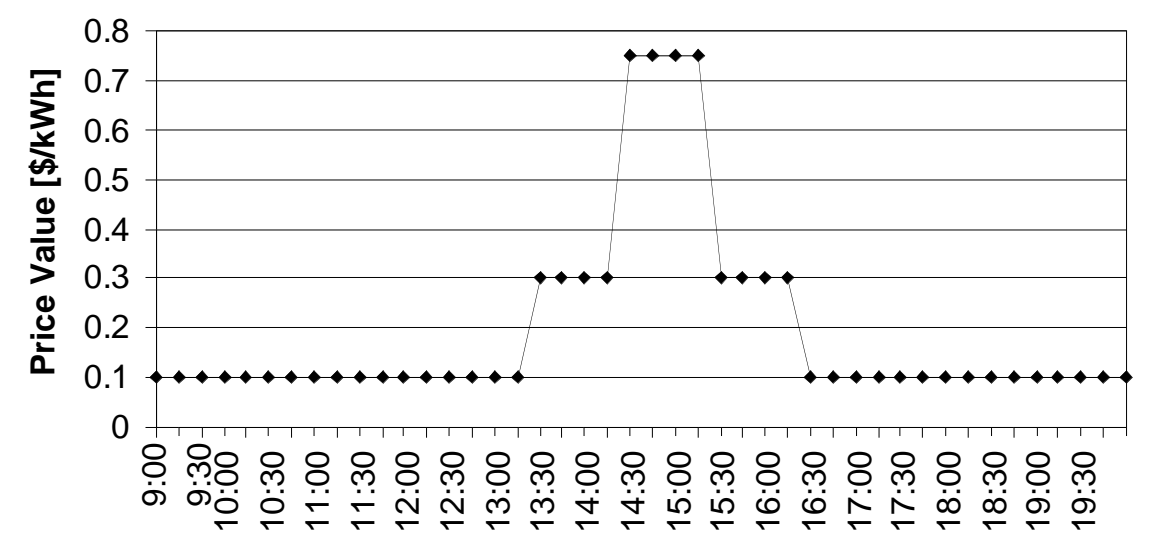

Figure 2-10. Test 2 Price Signal (November $19^{\text {th }}, 2003$ )

\section{2-7 Assessment of Lead Users of Auto-DR}

While the primary objective of this study was the development and evaluation of controls and communications systems for Auto-DR, we were also interested in learning about the decision-making processes and perspectives of the organizations and institutions recruited to participate. One important function of technology research is to accurately understand user needs for potential new technologies. As previous studies have stated, such understanding is clearly an essential input to the new technology development (Urban and Von Hipple, 1988). Technology users have a great deal to contribute and can assist in characterizing technology needs and the feasibility of new technologies. Section 
5 of this report discusses the lessons learned from using the Lead user concept in evaluating the needs and methods to promote DR in large facilities.

Lead users have been identified as an extremely valuable cluster of customers and potential customers who can contribute to the identification of future opportunities and evaluation of emerging concepts. Lead users are defined as organizations that exhibit the following two characteristics:

- They face the general marketplace needs months or years before the bulk of the marketplace encounters them.

- They are positioned to significantly benefit from obtaining solutions to those needs.

The managers of these types of programs realize that the solutions to energy problems are beyond the ability of one company or facility to solve. They are willing participants in this research project primarily because they understand the problems that could occur if facilities need to be shut down in an energy emergency. It was early in the development of an Auto-DR program for their facilities, but managers were willing to undergo extensive interviews and work together cooperatively with the research team to conduct the Auto-DR test and take part in the Lead Users evaluation.

The Lead User evaluation included a series of interviews with each of the key facility managers and owners. These interviews took place during the recruitment process. The Auto-DR organizational study methodology was primarily telephone interviews of the participants at the facility and the vendors who serve the facilities. The participants are located at some distance from each other and much of the work they do with the sites is via telephone and email. Shockman et al (2004) describe the results of the organizational investigation. 


\section{Auto-DR Systems Characterization and Measurement}

In the Auto-DR test, each of the five participating sites used different approaches in the design and implementation of their systems. This section examines the general characteristics of these systems. Where possible, metrics are used to quantify various attributes and characteristics of the Auto-DR systems. Quantifiable Auto-DR metrics are shown in tables following each section.

\section{3-1 Auto-DR System Architecture}

\section{An Overview}

Some Auto-DR facilities hosted the polling client software on-site and others hosted it at remote co-location sites (see Table 3-1). The geographic location of the computer that hosts the polling client is less important than the type of environment where it is hosted. Professional co-location hosting services, or "co-los" offer highly secure environments for hosting computers and servers. Co-los generally provide battery and generator backed electrical systems, controlled temperature and humidity, seismic upgrades and $24 / 7$ guarded access control. For companies that don't have similarly equipped data centers, co-los fill an important need. For computer applications where high availability is important, co-location facilities are often used.

Systems with a high level of integration between enterprise networks and EMCS networks tend to allow direct access to any or all control points in the EMCS without excessively labor intensive point mapping required. Direct remote control of EMCS points from enterprise networks means that the business logic computer has the ability to send commands over the network(s) that extend all the way to the EMCS I/O controller connected to the equipment that is shed. In a highly integrated system, the EMCS becomes an extension of the enterprise. In these types of integrated systems, a gateway device is used to translate between the different protocols used in enterprise networks and EMCS networks.

Alternately, some systems used relay contacts to interface between enterprise networks and EMCS networks. Relay contacts are commonly used in EMCS programming to define mode changes (e.g., smoke detector contacts). However, the use of relay contacts as an interface between networks is not very flexible. Modifications to shed strategies or other types of remote control would be more difficult with a relay interface system than with an integrated system with a translating gateway. Both gateway based and relay based interfaces to EMCSs can be effective for initiating shed strategies. 
Table 3-3. Characteristics of Auto-DR Systems - Architecture

\begin{tabular}{|l|c|c|c|c|c|}
\hline & Albertsons & B of A & GSA & Roche & UCSB \\
\hline Client hosted at co-lo & Yes & Yes & No & No & No \\
\hline Remote polling client & Yes & Yes & Yes & No & No \\
\hline $\begin{array}{l}\text { Remote control via } \\
\text { Internet Gateway }\end{array}$ & No & Yes & No & Yes & No \\
\hline
\end{tabular}

\section{Gateway Type}

Gateways used in building telemetry systems provide several functions. First, they connect two otherwise incompatible networks (i.e., networks with different protocols) and allow communications between them (see Figure 3-1). Second, they provide translation and usually abstraction of messages passed between two networks. Third, they often provide other features such as data logging, and control and monitoring of I/O points.

Of the five Auto-DR sites, two used embedded two-way communicating gateways to connect each site's EMCS networks to its enterprise networks (Table 3-2). Embedded devices are generally preferred over PC based gateway solutions for scalable, ongoing system deployments. Embedded devices have the following advantages:

- More physically robust. There are no hard drives or other moving parts.

- Less susceptible to viruses and other types of hacker attacks due to custom designed operating systems and applications.

- Less susceptible to human error. Once they are set up to function, there is no reason for site personnel to interact with the device. Since they are not "general purpose" computers, there is no risk of memory overloads due to computer games, screen savers and other applications that may be inadvertently loaded onto them.

- Better form factor. Embedded devices are usually smaller than PCs and are designed to be mounted in secure server rooms with other IT equipment.

- Lower cost. Although volume dependent, application specific embedded devices can be produced in volume for lower cost than PCs.

At Albertsons, an embedded IP I/O device (Engage EPIM ${ }^{\mathrm{TM}}$ ) was used for power monitoring and shed mode control. The EPIM provided power monitoring by directly counting pulses from power meters. The EPIM set various shed modes into operation by opening and closing onboard relay contacts. Although the EPIM IP I/O device effectively provides the interface between the EMCS and enterprise networks, it does not fit the most basic definition of a gateway. It does not connect the protocols of the two networks.

At UCSB, gateway functionality for monitoring was provided by software running on a PC. A previous version of gateway software also provided remote control functionality, but this feature was unavailable at the time of the Auto-DR test due to incompatibility issues that occurred after a software upgrade. To meet the remote control requirements 
of the Auto-DR test, an embedded IP I/O device was added. This device had onboard relay contacts similar to the EPIM, but direct measurement of I/O points (such as power meters) was not required.

Table 3-4. Characteristics of Auto-DR Systems - Gateways

\begin{tabular}{|l|c|c|c|}
\hline Albertsons & Gateway type & $\begin{array}{c}\text { Interface Device } \\
\text { Description for } \\
\text { Remote Shed Control }\end{array}$ & $\begin{array}{c}\text { Remote Monitoring } \\
\text { Description }\end{array}$ \\
\hline \hline B of A & Embedded IP I/O & IP Relay (2 contacts) & $\begin{array}{c}\text { Meter pulses are } \\
\text { monitored through } \\
\text { gateway }\end{array}$ \\
\hline GSA & $\begin{array}{c}\text { Embedded IP I/O } \\
\text { device }\end{array}$ & IP Relay (2 contacts) & $\begin{array}{c}\text { Monitoring of EMCS } \\
\text { through gateway }\end{array}$ \\
\hline Roche & $\begin{array}{c}\text { Embedded Gateway } \\
\text { Internet gateway }\end{array}$ & $\begin{array}{c}\text { Control of EMCS via } \\
\text { Internet gateway }\end{array}$ & $\begin{array}{c}\text { Monitoring of EMCS } \\
\text { through gateway }\end{array}$ \\
\hline UCSB & $\begin{array}{c}\text { PC based Gateway } \\
\text { for monitoring, } \\
\text { Embedded IP relay } \\
\text { for control }\end{array}$ & $\begin{array}{c}\text { IP Relay (3 contacts) } \\
\text { monitored through } \\
\text { gateway }\end{array}$ & $\begin{array}{c}\text { Selected EMCS points } \\
\text { mbongh }\end{array}$ \\
\hline
\end{tabular}

Figure 3-1, "Network architecture overview of five combined Auto-DR sites" shows the common source of electricity price and the communications protocol translations between the business logic and the final control element (relays, valves etc.) controllers that actually shed the electric loads. Gateways or other devices are used to transfer necessary communications between dissimilar network protocols. 


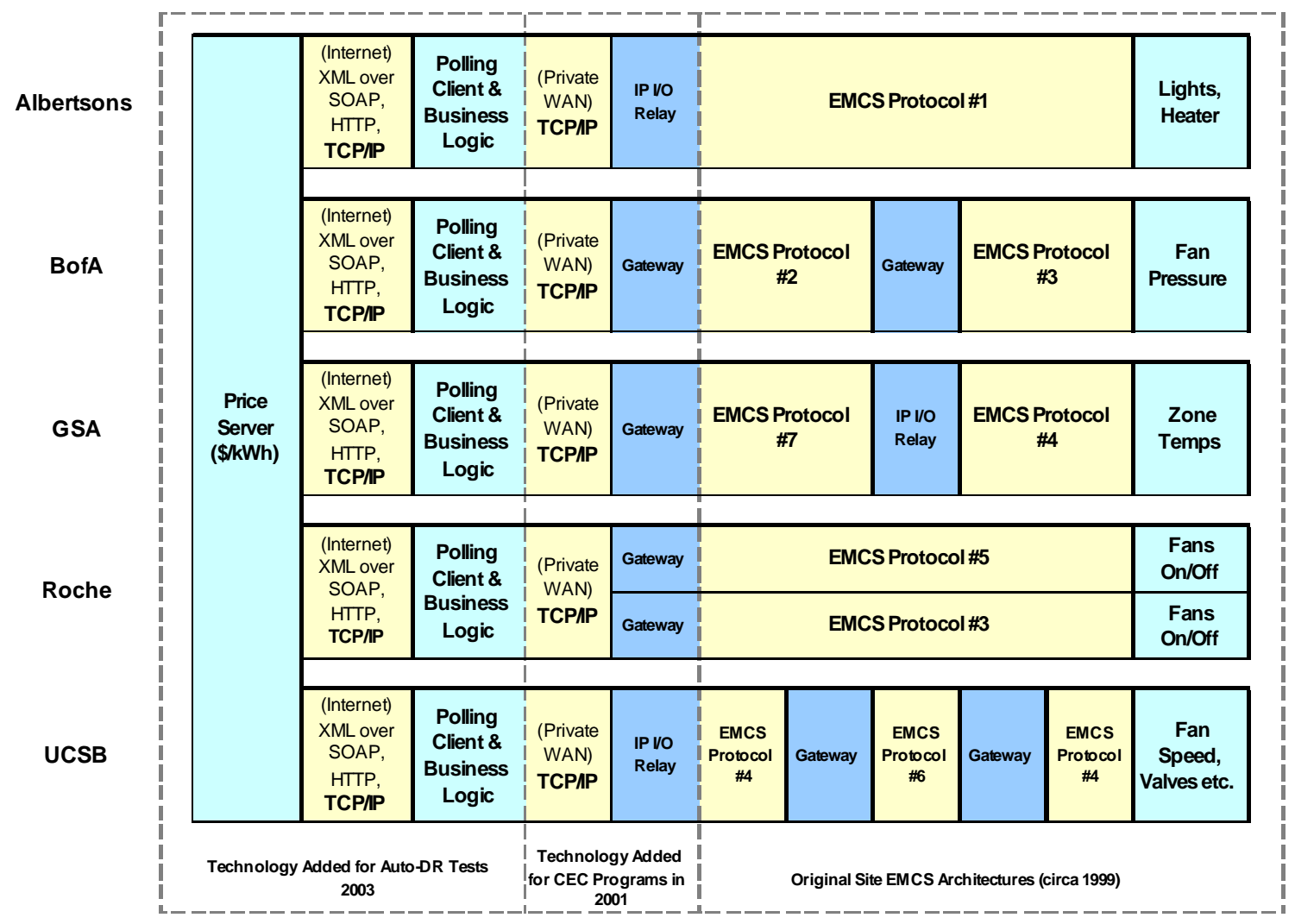

Figure 3-11. Network architecture overview of five combined Auto-DR sites

\section{Integration}

For purposes of this study, integration between EMCS and EIS can be characterized by asking two key questions. First, can data from the EMCS and EIS be viewed and analyzed from one Human Machine Interface (HMI)? Second, do the EMCS devices such as energy meters reside on the same network as the EMCS devices? Table 3-3 summarizes the answers to these questions for each of the sites. Albertsons, B of A, and GSA either don't have EIS or else they are not integrated with the EMCSs at those sites. At Roche, the Tridium system integrates most of the EMCS points and a small percentage of the electric meters into a comprehensive HMI for viewing, archiving and analysis. UCSB has extensive monitoring of most of the electric meters and sub-meters throughout the campus. Data from these meters are available for visualization, archiving and analysis through the SiE (Itron / Silicon Energy) server along with relevant EMCS points that have been mapped over to it. Electric meters do not reside on the same network as the EMCS devices at either the Roche or UCSB sites.

One distinguishing characteristic of the Auto-DR sites was whether or not they leveraged the existing corporate or campus enterprise network to transmit EMCS and/or EIS data. Use of the existing enterprise network for this purpose has many advantages. System installation costs can be much lower if existing enterprise networks are used for communications instead of installing new, separate networks solely for EMCSs and EISs. 
In addition, the Information Technology department that manages the enterprise is often better equipped to assure network reliability and security than the facilities group that traditionally maintains the EMCS and EIS.

Each facility has different functional requirements and organizational structures that dictate how the enterprise, EMCS and EIS networks are designed, installed and maintained. Of the five sites in the Auto-DR test, three of them shared mission critical enterprise networks with EMCS/EIS/Auto-DR systems. Although bandwidth requirements for EMCS/EIS/Auto-DR systems are not great, other organizational impediments prevent the sharing of enterprise networks for non-standard purposes.

At GSA, a completely separate enterprise network was created for the GEMnet EMCS/EIS/Auto-DR system. This was the logical choice for this facility because it was not practical to share the existing enterprise networks with other tenants at the site: the Government Services Administration (GSA) and the Federal Bureau of Investigation (FBI). In such circumstances, it is easier to create a new enterprise network for local and remote access to EMCS and EIS data than it is to resolve complex security and maintenance issues associated sharing an enterprise network with another department or organization. 
Table 3-5. Characteristics of Auto-DR Systems - Integration

\begin{tabular}{|c|c|c|c|c|c|}
\hline & Albertsons & B of $\mathbf{A}$ & GSA & Roche & UCSB \\
\hline Integrated EMCS \& EIS & No & No & No & Partial & Partial \\
\hline $\begin{array}{l}\text { Primary enterprise network } \\
\text { shared with } \\
\text { EMCS/EIS/Auto-DR } \\
\text { systems }\end{array}$ & Yes & Yes & No & Yes & Partial \\
\hline
\end{tabular}

\section{Shed Control Characteristics}

Each Auto-DR site used different shed strategies. The control characteristics of these strategies also varied substantially. This section describes the characteristics of each shed strategy (Table 3-4). The number of shed control points is one characteristic of a given Auto-DR implementation. It represents the quantity of points that were adjusted or altered to invoke the shed strategy at each site. Shed control points include hardware control points (for example, valve position) and software points (for example, setpoints) that were altered during the shed. Software points other than setpoints were not included. Control granularity and closed loop shed control are both factors that influence the likelihood and degree to which some occupants may be negatively affected by the shed strategy.

Albertsons had only two control points (overhead lights \& anti-sweat heaters). Because the size of the store is comparatively small, this was rated as "medium" control granularity. Switching off half of the overhead lights is an open loop type of control (i.e., there is no feedback to the system). The anti-sweat heater remained in closed loop control during the shed by operating with a reduced setpoint.

B of A had just one control point (duct static pressure) for the entire 211,000 $\mathrm{ft}^{2}$ building, hence the "coarse" control granularity rating. The shed strategy of resetting the duct static pressure so as to maintain zone temperature is a type of closed loop control, but the dearth of temperature sensors in the zones reduced the closed loop rating to "partial".

In stark contrast to the rest of the sites, the GSA building used a fine granularity, closedloop shed control strategy. The zone temperature setpoints for each of 1,050 VAV terminal boxes (1,400 including reheat side of dual duct boxes) were "relaxed" during the shed. In other words, the cooling setpoints were raised and the heating setpoints were lowered. This approach had an energy saving effect on the central HVAC systems while assuring a known level of service modification to the occupants.

The Roche site used a coarse open loop shed strategy of shutting off fans during the shed.

UCSB used a variety of shed strategies of medium granularity. The shed strategies (including closing cooling valves, and reducing duct static pressure) were all open loop. The outside air dampers were opened to $100 \%$, a strategy that could backfire in extremely hot conditions. The temperate climate in Santa Barbara made this scenario unlikely. 
Table 3-6. Characteristics of Auto-DR Systems - Shed Control

\begin{tabular}{|l|c|c|c|c|c|}
\hline & Albertsons & B of A & GSA & Roche & UCSB \\
\hline \hline $\begin{array}{l}\text { Number of } \\
\text { Shed Control Points }\end{array}$ & 2 & 1 & $\sim 1,400$ & 7 & 42 \\
\hline $\begin{array}{l}\text { Shed Control } \\
\text { Points per 10,000 ft. }\end{array}$ & 0.4 & 0.05 & 14 & 0.4 & 1.4 \\
\hline Control Granularity & Medium & Coarse & Very Fine & Medium & Fine \\
\hline $\begin{array}{l}\text { Closed loop shed } \\
\text { Control }\end{array}$ & Partial & Partial & Yes & No & No \\
\hline
\end{tabular}

\section{Open Standards}

In the EMCS and EIS fields, protocols refer to the low-level communication languages that devices use to "talk" to one another on the network. Of course, one device can only talk to another if they are speaking the same language. Traditionally, each control system manufacturer built controllers and other devices that only spoke their own unpublished proprietary protocol (Table 3-5). Once a system is built using a proprietary protocol, the original manufacturer or their representatives are the only parties that can make substantial additions or changes to the system. Some control companies use proprietary protocols as a "lock" on their customers' systems so as to ensure future business and high profit margins.

Over the past fifteen years or so, there has been a movement toward "open" protocols in the EMCS and EIS industries. Open protocols are based on published standards open to the public. Interested companies can build products that communicate using open standards. In a truly open, interoperable system, products from a variety of open product vendors could be added at any time by skilled installers from independent companies. Several sites in the Auto-DR test use open EMCS and/or EIS products that include the BACnet, LonTalk (EIA-709) and Modbus open protocols.

Even with considerable interest from building owners few, if any, new or existing building EMCS or EIS systems are truly open and interoperable. Even when open protocols are used, they are often installed as part of a system that requires use of proprietary software or components at the higher levels of the system architecture. Another way "openness" is reduced is by designing products and systems that require proprietary software tools for installation.

In the IT marketplace, open protocols (e.g., TCP/IP), open database interface standards (e.g., $\boldsymbol{O D B C}$ ) and open hardware standards (e.g., SIMM) have helped the industry thrive. This has allowed products from a wide variety of vendors to communicate with one another on internal LANs, WANs and the Internet. A service industry of independent Systems Integrators has grown to fill the need of integrating multiple vendor networks into cohesive systems.

Another important trend in the IT industry is the use of a new set of open standards, protocols and languages collectively known as XML/Web services. The use of $\mathrm{XML} / \mathrm{Web}$ services in the building controls industry is increasing. This trend will tend to 
increase the ability to easily distribute, share and use data from disparate EMCS, EIS and other business systems. This will create opportunities for new products and services that will improve comfort and efficiency in buildings.

In the Auto-DR test, the use of XML/Web services over the Internet provided an overarching open-standards platform by which all of the proprietary and partially open EMCS and EIS systems could communicate. Although the number of commands transmitted between the systems in the 2003 test was minimal (e.g., price, shed mode, etc.), the implications of XML based "add-on" interoperability are very powerful.

Table 3-7. Characteristics of Auto-DR Systems - Open Standards

\begin{tabular}{|l|c|c|c|c|c|}
\hline & Albertsons & B of A & GSA & Roche & UCSB \\
\hline \hline Open Protocol EMCS & No & Yes & No & Partial & No \\
\hline Open Protocol EIS & Yes & Partial & NA & Partial & No \\
\hline Open Protocol Auto-DR & Yes & Yes & Yes & Yes & Yes \\
\hline $\begin{array}{l}\text { Open Standards Auto- } \\
\text { DR }\end{array}$ & No & No & No & No & No \\
\hline $\begin{array}{l}\text { Data Archiving in } \\
\text { Open Database }\end{array}$ & NA & NA & Yes & NA & NA \\
\hline
\end{tabular}

NA $=$ Not Available

\section{Site Characteristics}

\section{Albertsons}

Albertson's system architecture is shown in Figure 3.2.

\section{EMCS}

Albertsons uses a proprietary protocol EMCS, Com-Trol, for control of the HVAC, lighting, refrigeration and freezer door anti-sweat heaters. Access to EMCS control and monitoring is available locally on the Human Machine Interface (HMI) or remotely through a dial-up modem.

\section{Enterprise Network}

Alberston's corporate WAN and the Internet are used to transmit energy data and AutoDR commands. Remote telemetry to electric data is provided via an IP I/O Device (Engage EPIM $\left.{ }^{\mathrm{TM}}\right)$. This device is capable of measuring electric data and remote monitoring over the enterprise network. In addition, the IP I/O device has digital outputs that can be controlled remotely over the enterprise network. A Web server that displays achieved data is hosted by eLutions at a co-location site in Tampa Bay, Florida.

\section{EIS and Measurement}

The EIS is from Engage/eLutions. Monitoring of electricity consumption is accomplished through pulse inputs from power meters. The pulses are converted to $\mathrm{kW}$ 
demand in the IP I/O device and accessed remotely through the eLutions Web based monitoring system. The Engage/eLutions EIS provides the following features:

- Monitoring: whole building and panel sub-metering.

- Visualization: Graphical charts including time-series, daily/monthly consumption summary, calendar profile, 3D profile, and duration curves.

- Data Archive: minute interval electric data are downloadable via the website.

- Other: The energy manager can select multiple Albertson sites and compare/rank their energy usages, though only one Albertsons site is featured in this test.

\section{Auto-DR System Description}

The computer containing the Auto-DR polling client and business logic is hosted at Elusion's co-lo in Florida. The business logic determines the correct operational mode for the Oakland store at any given time (normal, shed level 1, shed level 2) based on price and other operational criteria. It commands the IP I/O device relays to open or close using a predetermined code that represents each operational mode. These relay contacts are monitored by the Com-Trol EMCS. Upon sensing the state of the relay contacts, the EMCS commands the overhead store lights and anti-sweat heaters according to the shed strategy. The following business logic was essential at this site. As the store manager's preferred to return the store to normal operation by the evening shopping rush, all store systems are forced to "normal" operation between 3:30 pm and 10:00 pm, regardless of the current price of electricity.

\section{Shed Strategy}

In normal mode, the overhead lights are all on (100\%) during occupied hours. The freezer door anti-sweat heaters are controlled so as to prevent the glass doors from fogging when opened. The anti-sweat heaters control system uses a pulse width modulation (PWM) scheme that controls the amount of heat supplied to the glass based on the measured dewpoint temperature in the store. At night the dewpoint temperature setpoint is changed so as to use less energy. Upon a rise in price to $\$ 0.30 / \mathrm{kWh}$, overhead lighting was reduced by $50 \%$ by switching off half of the fluorescent tubes in each fixture. Upon a rise in price to $\$ 0.75 / \mathrm{kWh}$, the anti-sweat freezer door heater control switched to "night mode" which uses significantly less energy. 


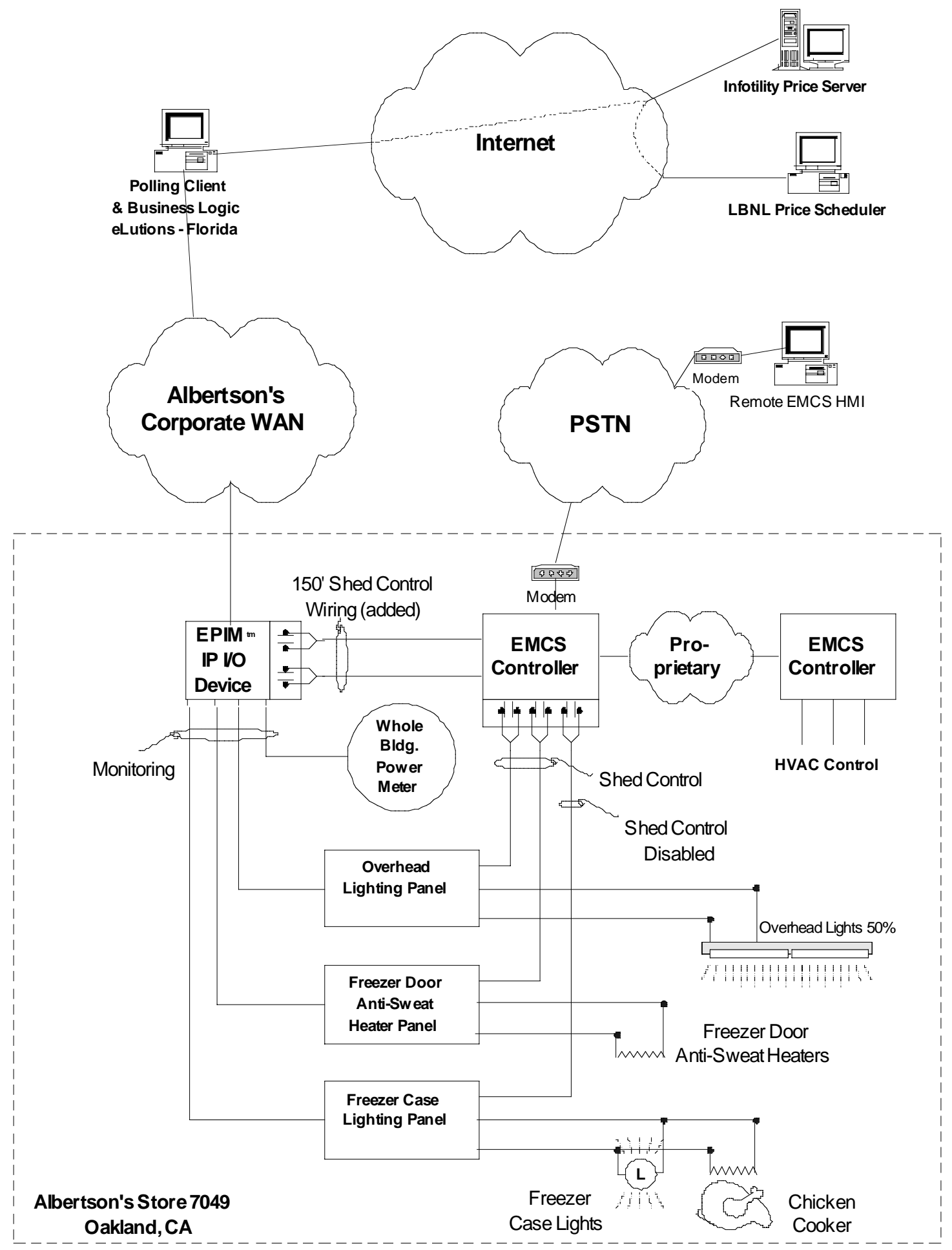

Figure 3-12. Albertsons - System Architecture 


\section{Bank of America Concord Technology Center (CTC)}

Bank of America's Concord Technology Center (CTC) system architecture is shown in Figure 3.3.

\section{EMCS}

The B of A CTC buildings involved in the Auto-DR test use an open protocol (LonTalk) system by Trane for control and monitoring of the HVAC equipment. Inter-building connectivity is accomplished through use of another open protocol (BACnet).

\section{Enterprise Network}

B of A uses the corporate WAN and the Internet for remote telemetry to the EMCS and EIS. Connectivity between the enterprise networks and the EMCS networks is provided by Tridium JACE ${ }^{\mathrm{TM}}$ gateways. The gateways connect the BACnet EMCS (including electric meters) to the B of A enterprise WAN. The EMCS, EIS and Auto-DR data are transferred across the same enterprise network infrastructure used for bank financial transactions. At the B of A Network Operations Center (NOC) in Chicago, communications between the B of A WAN and the WebGen Polling Client / Business logic computer in Andover MA is established across the public Internet. Cisco Virtual Private Network ( $\boldsymbol{V P N})$ routers were used at both ends of the secure VPN "tunnel". WebGen also provided a Web server, hosted at the co-location site in Andover, Massachusetts, for visualization of the EMCS and EIS data.

\section{EIS and Measurement}

For the Auto-DR test, monitoring, archiving and visualization of energy data were accomplished through the use of three separate systems: PG\&E (Interact), WebGen (IUE), and temporary sub-metering equipment.

InterAct is a Web based, whole site EIS provided by PG\&E for large commercial customers. At the B of A CTC, site demand, individual building interval data, and weather data from NOAA can be monitored and trended. The data can be visualized in the following formats: Time-series, daily/monthly consumption summary, scatter plot, histogram and average daily profile. IUE's features include control, monitoring and data visualization. The control characteristics implemented for the Auto-DR test are described below. This system is also described in Motegi et al, 2003a. Since the existing site EIS systems did not measure electric data at the level of resolution required for the Auto-DR test, additional temporary sub-metering equipment was added.

\section{Auto-DR System Description}

WebGen system is designed to operate as an ongoing demand and energy reduction system. To save energy, the WebGen IUE system intermittently takes setpoint control away from local operators and gives control to the automated system in Andover. During these "interventions", duct static pressure setpoint is reduced on one VAV fan system at a time. The setpoint reduction was rotated between fans so as to share the reduction is service throughout the site. 
The computer containing the Auto-DR polling client and business logic was hosted at WebGen's co-lo in Andover, MA. The business logic contains learning algorithms designed to maintain comfort while reducing energy costs. The Auto-DR price signal was intended to further reduce energy use.

WebGen has a unique feature that estimates the savings (dollars and $\mathrm{kWh}$ ) that are realized each time the WebGen system causes control to vary from normal operation. At the B of A CTC site, sensors are not installed for fan power, fan airflow, or pressure drop across fans. Since data necessary to calculate the energy savings per intervention are not measured at this site, it is not possible for the WebGen system to make accurate estimates on a "per intervention" basis.

WebGen's site wide financial savings calculations are made using revenue grade electric meters. Savings are calculated as the difference between the measured whole site electric meter values (with shed interventions) and what the electric consumption "would have been" without the sheds. These calculations may be accurate within the constraints of normal regression analysis based on current standards of practice such as those used in the International Performance Measurement and Verification Protocol (IPMVP).

\section{Shed Strategy}

As fan duct static pressure setpoints are reduced to save fan energy, sensor data (temperatures, pressures, etc.) are evaluated. The system is designed to "learn" the effect reductions in the duct static pressure has on zone temperatures so as to minimize negative

comfort impacts. Artificial Intelligence algorithms are used in these calculations. During the Auto-DR test, the system responded by reducing duct static pressure by 0.2 " W.C. for 20 minutes per fan. Pressure reductions were rotated between fans in different buildings to maintain comfort and shed demand at the same time. 


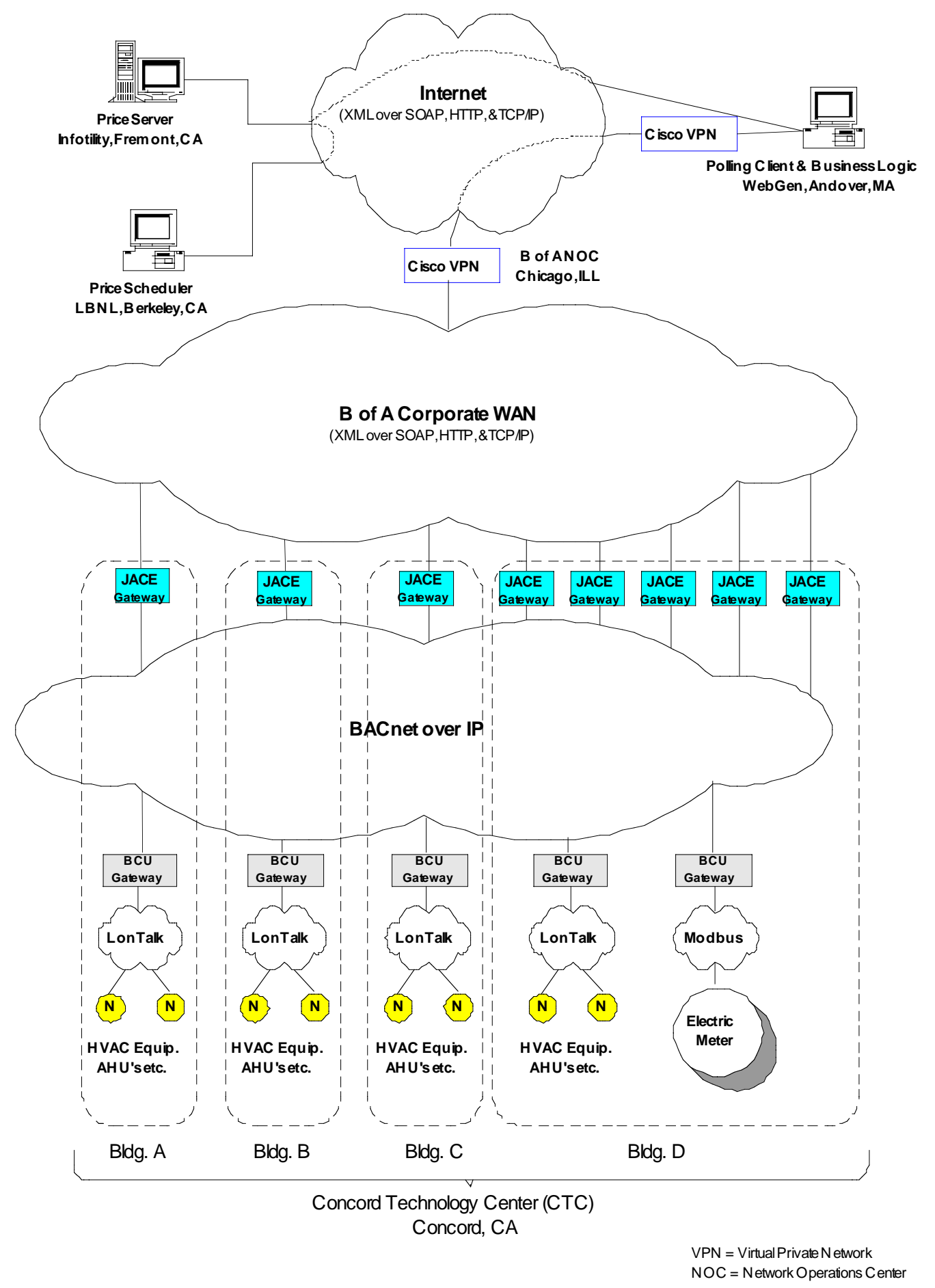

Figure 3-13. B of A-System Architecture 


\section{General Services Administration - Oakland Federal Building, Oakland}

The Oakland Federal Building system architecture is shown in Figure 3.4.

\section{EMCS}

A Johnson Metasys proprietary protocol EMCS is used to control the VAV fan system and central heating and cooling plants. Although they are not used for HVAC control, several Alerton BACnet open protocol devices were used to facilitate Auto-DR communications.

\section{Enterprise Network}

The Oakland GSA site is connected to an innovative enterprise network designed to improve the efficiency and operations of the connected buildings. GEMnet (GSA Energy and Maintenance Network) is being developed and implemented for energy management and building maintenance in over a dozen Federal buildings in the Pacific Rim Region. The enterprise network and DSL Internet connectivity were designed and implemented by controls and IT specialists within the facilities group of GSA.

\section{EIS and Measurement}

Monitoring, archiving and visualization of energy data were accomplished through the use of three separate systems: PG\&E (Interact), EMCS trend logs, and temporary submetering. The Interact system monitors five interval meters throughout the building that collectively measured whole building demand and energy. Temporary sub-metering was added to this site to monitor four cold deck supply fans and two secondary chilled water pumps.

\section{Auto-DR System Description}

In the Auto-DR test, a polling client in Kelowna, British Columbia (BC) polled the Infotility price server in Fremont. Upon a rise in price, the business logic in the BC computer posted a value that represented shed mode (normal, shed level 1, shed level 2) to the main GEMnet SQL database. A custom software application called BACnet reader polled the SQL database every 2 minutes. When the mode value changed to a shed mode, BACnet reader commanded an Alerton I/O controller with 2 digital outputs (via a software gateway by PolarSoft and an Alerton LSI TCP/IP to BACnet MSTP gateway) to close either one or two relay contacts. An I/O controller with two digital inputs read the relay contacts. The shed strategy was then implemented throughout the Johnson EMCS.

\section{Shed Strategy}

GSA employed a global zone temperature setpoint setup/setback shed strategy (Figure 36). Upon commencement of a shed (due to price increase), the zone temperature setpoints for all of the individual zones in the building were "relaxed". In other words, if fan and cooling energy is being used to keep a given zone from rising above $74^{\circ} \mathrm{F}$, a new setpoint is issued to keep it from rising above $76^{\circ} \mathrm{F}$. Similarly, zones that have heating setpoints are relaxed to allow zones to get slightly cooler before using more energy. When this approach is applied to all of the zones in the building, substantial energy can be saved. 
Table 3-8. Oakland GSA Zone Temperature Setpoints - Normal and Shed Modes

\begin{tabular}{|c|c|c|}
\hline Shed Mode & $\begin{array}{c}\text { Zone Heating } \\
\text { Setpoint }\end{array}$ & Zone Cooling Setpoint \\
\hline Normal & $70^{\circ} \mathrm{F}$ & $72^{\circ} \mathrm{F}$ \\
\hline $\begin{array}{c}\text { Level 1 } \\
(\mathbf{\$ 0 . 3 0 / k W h )}\end{array}$ & $68^{\circ} \mathrm{F}$ & $76^{\circ} \mathrm{F}$ \\
\hline $\begin{array}{c}\text { Level } \mathbf{2} \\
(\mathbf{\$ 0 . 7 5 / k W h})\end{array}$ & $66^{\circ} \mathrm{F}$ & $78^{\circ} \mathrm{F}$ \\
\hline
\end{tabular}




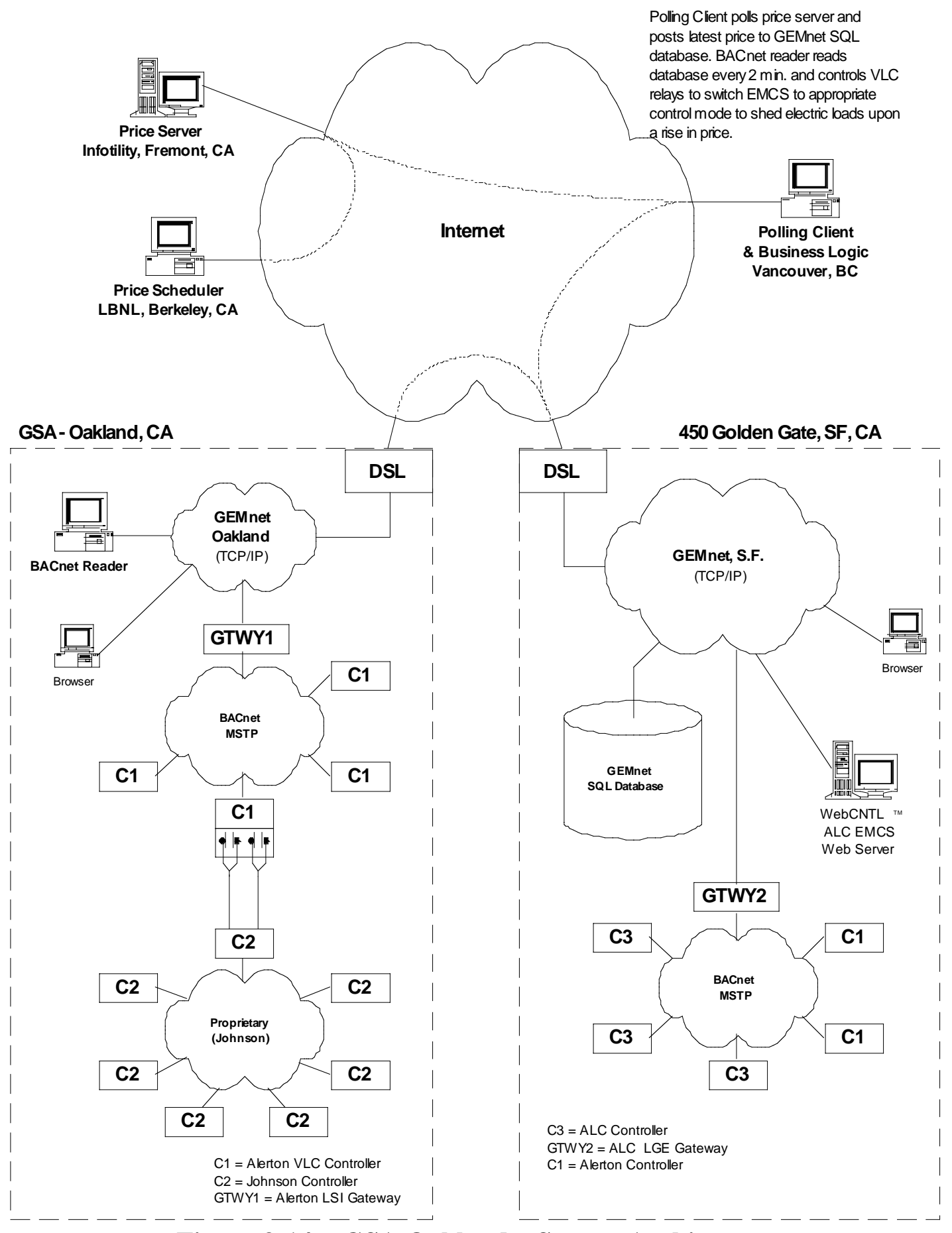

Figure 3-14. GSA Oakland - System Architecture 


\section{Roche Pharmaceuticals Palo Alto}

The Roche Pharmaceuticals system architecture is shown in Figure 3.5.

\section{EMCS}

The EMCS at the Roche site is comprised primarily of Invensys products. There are at least two generations of Invensys products used: Network 8000 (proprietary protocol RS-485) and the I/A product line (open protocol LonTalk). In addition, open protocol Modbus and proprietary RS-485 (different from the Network 8000) are used for electricity monitoring. These diverse systems are tied together into one common user interface and database by the Tridium Niagra framework. The Tridium system uses embedded gateways to translate from each aforementioned EMCS protocol to TCP/IP.

\section{Enterprise Network}

The Tridium system provides password-protected access of the EMCS data via any Web browser within the Roche corporate intranet. Due to security concerns and lack of compelling reason to do otherwise, the Tridium EMCS/EIS Web browser is not accessible from the public Internet.

Due to security concerns, the polling client was installed just outside of the Roche secure intranet in a $\boldsymbol{D M Z}$ region $^{8}$. A special virtual connection was made between the Tridium computer inside the secure Roche intranet and the polling client computer that is located in the DMZ. This allows the polling client easy, two-way communication with other computers in the public Internet, while not exposing the Roche network to security concerns associated with the Internet.

The polling client computer also served another purpose. To aid in the data collection requirements of the Auto-DR project, about 40 EMCS points were extracted from the Tridium system and logged and archived on a computer outside of the Roche secure network. These data were made available to LBNL researchers in Berkeley in near real time.

\section{EIS and Measurement}

Monitoring, archiving and visualization of energy data were accomplished through the use of three separate systems: Tridium (Vykon ${ }^{\mathrm{TM}}$ ), Pegasys (PML ${ }^{\mathrm{TM}}$ ), Infotility RealTime Energy Tracker (RTET). Tridium Vykon provided Internet based control and trending for Roche inside their firewall. RTET is a real-time Infotility Web-monitoring tool designed specifically for this project. It was developed to overcome the remote data access problems related to Roche's Internet firewall. RTET was used to transmit energy data from the Tridium computer to the Infotility server where it could be accessed remotely by project researchers. The Pegasys system provided electricity, gas, steam and water metering. It was used to obtain data for three buildings (A2, SS and FS). The PEGASYS PML is a closed-network system that is only accessible within the site.

\footnotetext{
8 "Short for demilitarized zone, a computer or small subnetwork that sits between a trusted internal network, such as a corporate private LAN, and an untrusted external network, such as the public internet", Webopedia. 2004. http://www.webopedia.com/TERM/D/DMZ.html. Viewed on Mar. 15, 2004 .
} 
Considerable effort was required to determine how to download and analyze the PML TM data.

\section{Auto-DR System Description}

No extra hardware was required to enable the Roche site's Auto-DR functionality. Communication from the price server to the EMCS I/O controllers occurred via networks. The increased price was sensed by the polling client and passed through to the Tridium system where it was read by the EMCS.

\section{Shed Strategy}

The shed strategy implemented at Roche is simple: when the price rises to $\$ 0.30 / \mathrm{kWh}$, several constant volume fans are shut off. When the price rises to $\$ 0.75 / \mathrm{kWh}$ several more fans were turned off. This strategy was very effective at immediately reducing electric load. It is also a rather severe measure to impose upon occupied areas, especially in hot weather. For the Auto-DR test, the fans that were selected for shedding and the strategy employed were carefully selected by building operators to minimize adverse effects on occupants.

The fan systems serving the selected areas have dual supply fans that operate in parallel. The return fan systems have a similar dual fan configuration. During the shed, only one of the fans in each parallel system was disabled. Because of this, about half of the normal air supply continued to flow into the buildings while the shed was in effect.

The fans involved in the shed serve the cafeteria. Since the test was conducted in the mid-afternoon, the lunchtime crowd was gone and the cafeteria had very few occupants. Similarly the facility's office and administration auditorium had low occupancy during the test. Zone temperature and system $\mathrm{CO}_{2}$ sensors indicate that there was a minimal impact on the comfort of the areas affected by the shed. Temperature did not measurably deviate from setpoint and $\mathrm{CO}_{2}$ level decreased during the shed. 


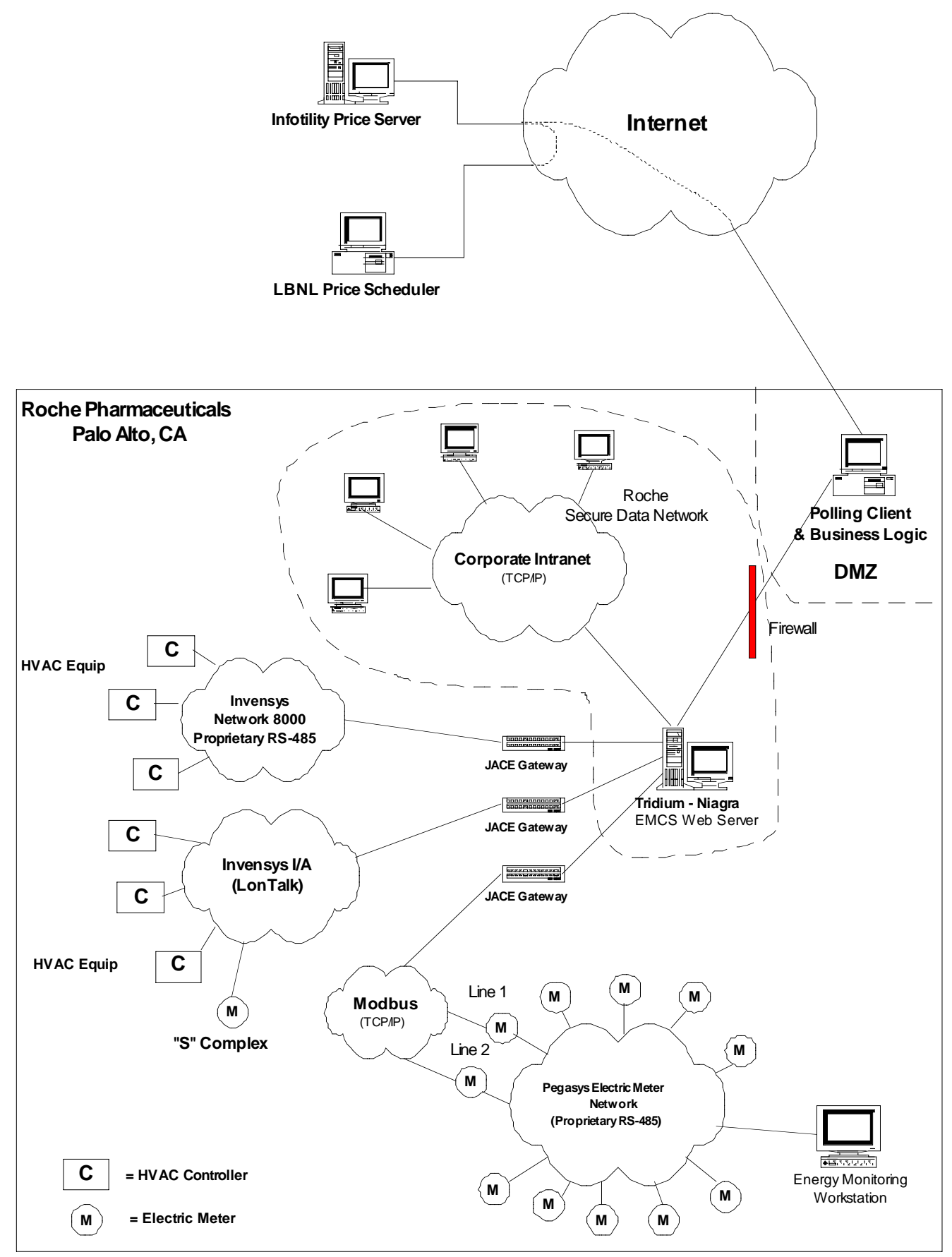

Figure 3-15. Roche - System Architecture 


\section{University of California Santa Barbara}

The University of California Santa Barbara system architecture is shown in Figure 3.6.

\section{EMCS}

A Johnson Metasys proprietary protocol EMCS is used to control the HVAC systems on the UC Santa Barbara campus. Two versions of the Johnson protocol are used: "N2" communications between I/O controllers within each building and the much faster "N1" which communicates between buildings over the campus intranet.

\section{Enterprise Network}

The facilities department has its own enterprise subnet, separate from UCSB campus network. This subnet includes Johnson gateways (TCP/IP to N1) and several computers used by the facilities department staff. The polling client and business logic software was installed on the existing SiE server which also resides on the facilities department subnet. An IP relay was added for the Auto-DR test.

\section{EIS and Measurement}

All of the data required for the project was collected through the Itron EEM Suite ${ }^{\mathrm{TM}}$, a Web-based EIS for monitoring and analyzing building energy data. EMCS data points can be mapped over to the EEM system so they can be visualized or analyzed within the same environment as the energy data. The scope of the monitoring for the Library II, III, and IV included electric power, gas consumption, cooling energy delivered to the building (Btu meter) and estimated chiller power (based on an average $\mathrm{kW} /$ ton).

\section{Auto-DR System Description}

In the Auto-DR test, a polling client polled the Infotility price server in Fremont. Upon a rise in price, the business logic directly commanded the IP I/O controller to close either one, two or three relay contacts. The number of contacts closed correspond directly to the three shed modes (normal, shed level 1, shed level 2). The relay contact positions were read by a Johnson I/O controller with three digital inputs.

\section{Shed Strategy}

The following shed strategy was performed at the UCSB Main Library:

As the electricity price rose to the following values, the associated measures were employed:

\section{- $\$ 0.30 / \mathrm{kWh}$}

Measures applied to three supply fans in the library complex:

Supply fan Variable Frequency Drives (VFD) were limited to $70 \%$ speed. Economizer mixing dampers were forced to $100 \%$ outside air.

\section{- $\$ 0.75 / \mathrm{kWh}$}

The speed of the three supply fans was further reduced to a $60 \%$ limit The speed of two additional supply fans was reduced by lowering the static pressure setpoint to 0.4 IWC. 
Economizer mixing dampers were forced to $100 \%$ outside air.

The heating and cooling valves of all five fans were forced to $0 \%$ (closed).

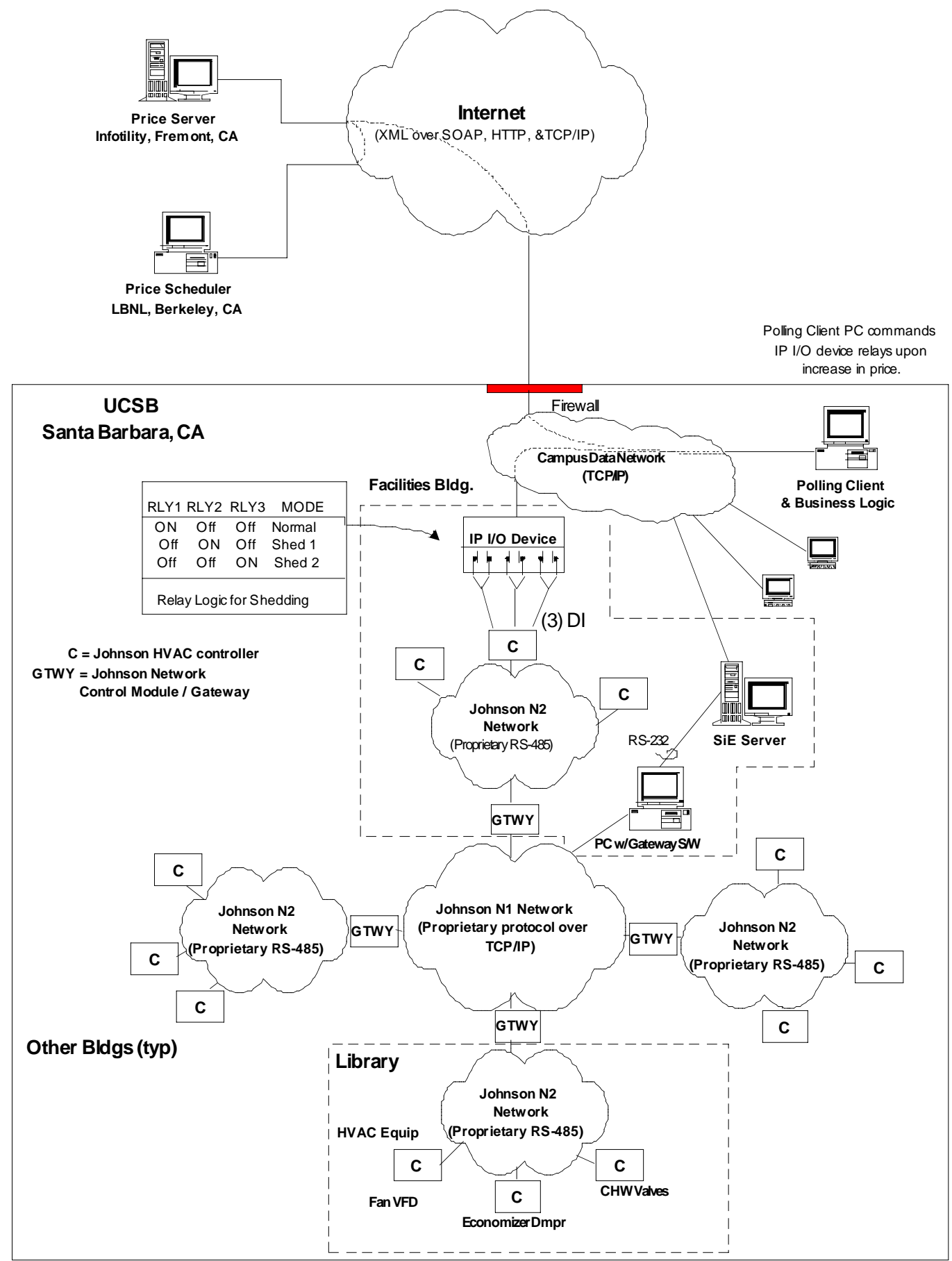

Figure 3-16. UCSB - System Architecture 


\section{3-2 Site Measurement and Evaluation Techniques}

This section describes the site measurement and evaluation techniques used to measure the electric demand shed. Measurement techniques were developed to evaluate each 15minute increment of the three-hour electric shed event. Where possible, we also developed non-energy measurements focusing on temperature or other indoor air environmental factors (see Section 4). The shed events were followed by interviews with facility operators to inquire about any loss of service or problem that may have resulted from the shed strategy (See Section 4 and Appendix V).

Numerous sources of data were used to measure and evaluate the load shedding. Table 37 summarizes the measurements at each site, showing the type of system, types of data collected, number of data points used from the system, frequency of data measurement, and whether it had Web-based data access. Detailed point lists are included in Appendix VI. All five sites had some form of an EIS. Multiple sources of data including EIS, EMCS, and submeter were used in four of the five sites. At Albertsons, only one EIS was used for data collection and analysis. At the four sites with multiple sources of data, significant re-configuration of the EMCS and EIS trending points was required.

Table 3-9. Measurement Summary

\begin{tabular}{|c|c|c|c|c|c|}
\hline Site & Type & Data type & $\begin{array}{c}\text { \# of points } \\
\text { used }\end{array}$ & $\begin{array}{l}\text { Data } \\
\text { freq. }\end{array}$ & $\begin{array}{l}\text { Web-based } \\
\text { access }\end{array}$ \\
\hline $\begin{array}{l}\text { Albert } \\
\text { sons }\end{array}$ & EIS & $\begin{array}{l}\text { WBP, Overhead lighting, } \\
\text { Anti-sweat heaters, OAT }\end{array}$ & 4 & 15 min. & Yes \\
\hline \multirow{4}{*}{$B$ of $A$} & EIS & $\begin{array}{l}\text { WBP, Fan (status, temp, VFD, } \\
\text { air flow, static pressure) }\end{array}$ & 35 & $5 \mathrm{~min}$. & Yes \\
\hline & EIS & WBP, OAT & 2 & $15 \mathrm{~min}$. & Yes \\
\hline & EMCS & $\begin{array}{l}\text { Chiller power, Chilled water energy } \\
\text { Control status }\end{array}$ & 21 & $15 \min$. & $\mathrm{No}^{*}$ \\
\hline & Submeter & $\begin{array}{l}\text { Meter installed: Fan power, } \\
\text { Individual building power }\end{array}$ & 8 & $1 \mathrm{~min}$. & No \\
\hline \multirow{3}{*}{ GSA } & EIS & WBP, OAT & 6 & 15 min. & Yes \\
\hline & EMCS & $\begin{array}{l}\text { WBP, Chiller power/energy } \\
\text { Fan (status, temp, VFD, air flow, } \\
\text { static pressure) } \\
\text { Cooling tower, Pump (status,VFD) } \\
\text { Zone temp }\end{array}$ & 100 & $\begin{array}{l}5-15 \\
\min .\end{array}$ & No \\
\hline & Submeter & $\begin{array}{l}\text { Meter installed: } \\
\text { Fan power, Pump power }\end{array}$ & 6 & $1 \mathrm{~min}$. & No \\
\hline \multirow{3}{*}{ Roche } & EIS/EMCS & $\begin{array}{l}\text { Newly trended EMCS points: } \\
\text { Fan status, Zone temp, } \mathrm{CO}_{2}\end{array}$ & 19 & $5 \mathrm{~min}$. & Yes \\
\hline & EIS & Individual building power & 3 & $15 \mathrm{~min}$. & No \\
\hline & Submeter & Spot measurement: Fan power & & & No \\
\hline \multirow[t]{2}{*}{ UCSB } & EIS & $\begin{array}{l}\text { WBP, Chilled water energy, OAT } \\
\text { Newly trended EMCS points: } \\
\text { Fan power, VFD, static pressure } \\
\text { Economizer position, Zone temp } \\
\text { Cooling and heating valve position }\end{array}$ & 150 & $\begin{array}{l}5-15 \\
\min .\end{array}$ & Yes \\
\hline & EMCS & Trends through EIS & & & No \\
\hline
\end{tabular}


WBP - whole building power; OAT - outside air temperature; VFD - variable frequency drive;

$\mathrm{CO}_{2}-$ carbon dioxide * Web access was not available to LBNL because of firewall security. 
Demand savings were derived by subtracting the actual metered electric consumption from the baseline consumption. The baseline consumption is an estimate of how much electricity would have been used without the demand shedding. We developed several methods to estimate baseline electricity consumption. Each building was evaluated using two methods: 1) a whole building, or top-down, and 2) a component level, or bottom-up method.

\section{Whole-Building Level Method}

The whole building, or premise-level, top-down, analysis method consists of developing a whole-building electrical power baseline load shape. Whole building power is estimated using a weather regression model that assumes whole building power is linearly correlated with outside air temperature (OAT). The OAT data is from local weather stations. Input data are 15-minute interval whole building demand and hourly OAT. This method was derived from previous work at LBNL (see KEMA-Xenergy, 2003). In the previous work, this method was tested with the GSA data, and statistically proven to be a better estimate than the conventional method of simple averages ${ }^{9}$ when the WBP has a linear correlation with OAT. The model is computed as;

$$
L_{15 \min }=a_{15 \min }+b_{15 \min } T_{h}
$$

where $L_{15 \min }$ is the predicted 15 -minute interval electric demand from the previous noncontrolled working days. The number of previous days used in the model was selected to make the best use of available metered data. Depending on frequency of available weather data, $T_{h}$ is the hourly or 15-minute interval OAT. $a_{15 \min }$ and $b_{15 \min }$ are estimated parameters generated from a linear regression of the input data. Separate regression equations are developed for each 15-minute interval, resulting in 96 regressions for the entire day (24 hours/day, with four 15-minute periods per hour). An example of the OAT linear regression model is given in Appendix II.

To develop the baseline electric loads for the demand sheds we selected 18 "non-shed" days. These 18 baseline days were non-weekend, Monday through Thursday workdays. Fridays were eliminated in the development of the baseline because the UCSB Library has a different operating schedule on Fridays. The selection of the baseline days was based on availability of whole-building data at each site. The submetering for Building B at Bank of America was installed on October $15^{\text {th }}$, which was the first day of the 18-day baseline period. The November $11^{\text {th }}$ Veterans Day holiday was not included in the baseline.

Figure 3-7 shows an example of the whole-building level baseline time-series chart on the second test day (GSA). The chart shows whole building power for the shed (the lower curve) and the whole-building baseline power predicted if the shed had not occurred. The vertical line at each baseline power data point is the standard error of the

\footnotetext{
${ }^{9}$ California Independent System Operator (Cal ISO)'s Demand Response Program used "previous 10 business days baseline", which is a simple average of previous days.
} 
regression estimate. The vertical lines at 1:15 pm, 2:15 pm, 3:15 pm, and 4:15 pm identifies time intervals during which price signals were different.

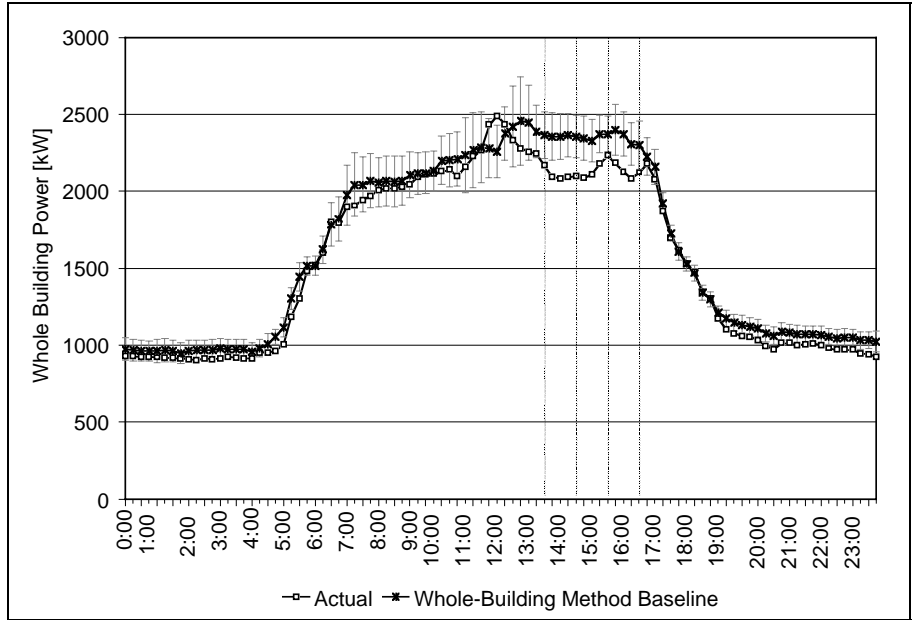

Figure 3-17. Example of Whole-Building Method Baseline

\section{Component Level Method}

The component level, or bottom-up evaluation method develops the WBP savings by combining the demand savings estimates for each component. The basic procedure is to: 1) develop a baseline for each controllable component, 2) estimate the demand savings for the component by subtracting actual demand from the baseline, and 3) sum all the controllable load demand savings. The component level estimation method requires analysis of either direct power measurement or operational data for each controllable component. The component level method is more accurate than the whole-building method if the controllable load and non-controllable load can be accurately measured. We developed several methods to develop the component level baseline load shape. These methods are described below.

\section{Equipment Schedule Method}

The controllable component "normal-day" operational load shapes can be used to define a baseline if the equipment schedule is well defined and consistent. This method can be applied to components with simple operational modes. This method is very accurate if the operation is simple enough to extrapolate electricity demand. A weather-sensitive component load can not be evaluated using this method. Examples of components that can be evaluated using this method include constant volume fans operating with a fixed schedule and non-dimmable lighting.

\section{Outside Air Temperature Regression Model Method}

If the component's electricity demand is weather sensitive, a simple average of the previous days' demand may provide a lower baseline demand if the controlled day's temperature is higher than that of the previous days. In this case, the estimate can be adjusted for OAT. This method forecasts the component demand by a regression model similar to the whole-building level method. The 15-minute component electric demand 
plus hourly OAT data are used. Examples of components evaluated with this method include chillers and fans with VFDs.

\section{Prior Time Load Method}

If the results of the equipment schedule or outside air temperature regression model methods do not accurately fit the actual demand data, the forecast demand may be estimated from the data at 15-minute to a few hours immediately preceding the test. This is a rule-of-thumb method. This method may be applied only when the equipment operation is stable without the shed strategies, and the daily load shape is similar over the previous days. Depending on the demand profile, the calculation method can be selected from either using previous operation hours or previous 15-minute demand, or drawing a line from the pre-shed demand and the post-shed demand. This method should be used when the shed period is short, and there is no shed strategy applied in advance (such as pre-cooling).

Table 3-8 summarizes the component-level savings estimation methods used for different components at each site. There are various reasons why different evaluation methods were used for the same type of equipment at different sites, due to load shapes and available data at each site.

Table 3-10. Component Level Methods for Each Component

\begin{tabular}{|c|c|c|c|c|}
\hline Site & Component & $\begin{array}{c}\text { Equipment } \\
\text { Schedule }\end{array}$ & $\begin{array}{c}\text { OAT } \\
\text { Regression }\end{array}$ & $\begin{array}{c}\text { Prior Time } \\
\text { Load }\end{array}$ \\
\hline \multirow{2}{*}{ Albertsons } & Sales lightings & $\checkmark$ & & \\
\hline & Anti-sweat heater & & & $\checkmark$ \\
\hline B of $A$ & Fan system & & & $\checkmark$ \\
\hline \multirow{4}{*}{$\begin{array}{l}\text { GSA } \\
\text { Oakland }\end{array}$} & Fans & & $\checkmark$ & \\
\hline & Chillers & & $\checkmark$ & \\
\hline & Pumps & & $\checkmark$ & \\
\hline & Cooling towers & & $\checkmark$ & \\
\hline Roche & Fans & $\checkmark$ & & \\
\hline \multirow{2}{*}{ UCSB } & Fans & & $\checkmark$ & $\checkmark$ \\
\hline & Chillers & & $\checkmark$ & \\
\hline
\end{tabular}

Figure 3-8 shows a component level method baseline time-series chart from the Roche site. The chart shows the fan energy, total building energy and component savings. 


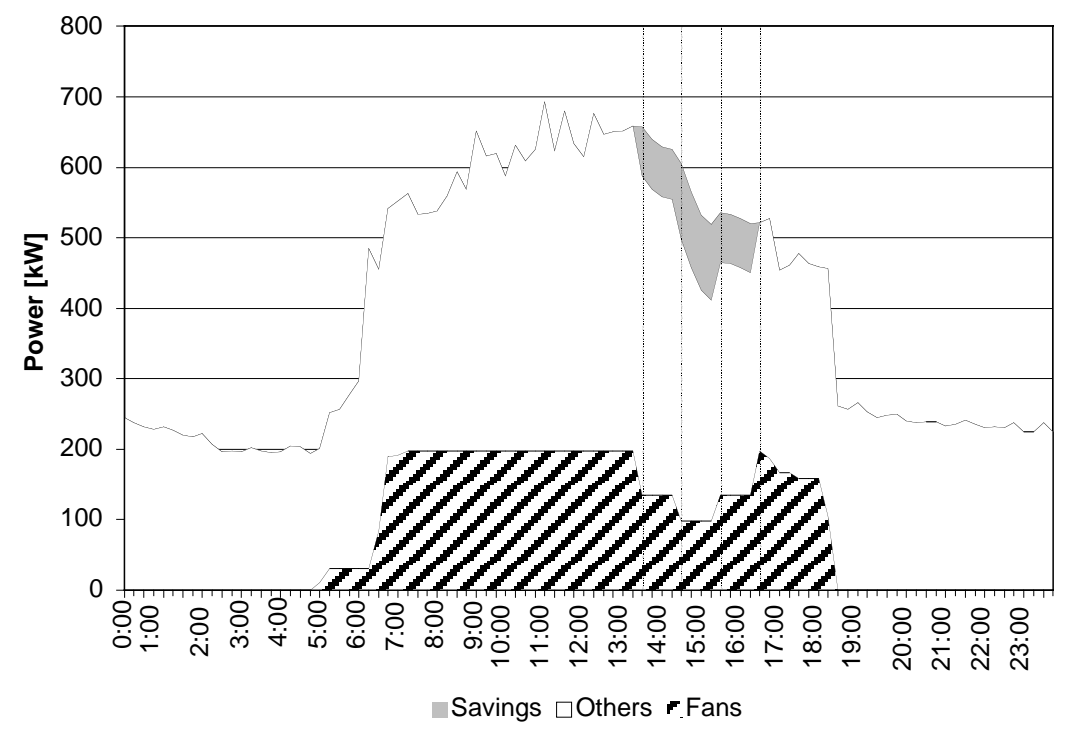

Figure 3-18. Example of Component Method Baseline - Roche 


\section{Results}

This section discusses the results of the Auto-DR tests, including: discussions regarding the receipt and confirmation of the XML price signal; discussion of the demand shedding measurements; and non-energy measurements.

\section{4-1 Requesting and Confirming Receipt of Signal}

Infotility developed a user interface for LBNL to verify communications between the polling clients at each site and the price server. Table 4-1 shows the communications recorded during the period when the price changed from $\$ 0.30 / \mathrm{kWh}$ to $\$ 0.75 / \mathrm{kWh}$ during the November $19^{\text {th }}$ Auto-DR test. From this interface, we verified that participants were polling of the correct price channel (identified as Price_LBNL1 channel in Table 4-1). We also could view the polling frequency of each participant and determine if they . interpreted the signal correctly ("Price sent by server" and "Price returned by user").

Table 4-11. Record of Prices Being Requested and Receipt Being Confirmed

\begin{tabular}{|c|c|c|c|c|c|c|c|c|}
\hline $\begin{array}{l}\text { ChannelID } \\
\underline{\underline{\text { [asc] }}}\end{array}$ & $\begin{array}{l}\text { Channel } \\
\text { Description }\end{array}$ & UserID & UserName & $\begin{array}{l}\text { When } \\
\text { requested by } \\
\text { user }\end{array}$ & Timestamp & $\begin{array}{l}\text { Price sent } \\
\text { by server }\end{array}$ & $\frac{\text { Price }}{\text { returned by }}$ & $\begin{array}{l}\frac{\text { When }}{\text { returned by }} \\
\text { user }\end{array}$ \\
\hline 1233 & Price_LBNL1 & 389 & gsa,cpu1_ & $\begin{array}{l}11 / 19 / 2003 \\
1: 45: 22 \text { PM }\end{array}$ & $\begin{array}{l}\text { 11/19/2003 } \\
\text { 2:00:00 PM }\end{array}$ & 0.3 & 0.3 & $\begin{array}{l}11 / 19 / 2003 \\
1: 59: 37 \mathrm{PM}\end{array}$ \\
\hline 1233 & Price_LBNL1 & 402 & boa,cpu1_ & $\begin{array}{l}11 / 19 / 2003 \\
1: 45: 30 \mathrm{PM}\end{array}$ & $\begin{array}{l}\text { 11/19/2003 } \\
\text { 2:00:00 PM }\end{array}$ & 0.3 & 0.3 & $\begin{array}{l}11 / 19 / 2003 \\
1: 46: 31 \mathrm{PM}\end{array}$ \\
\hline 1233 & Price_LBNL1 & 385 & ucsb,cpu1_ & $\begin{array}{l}11 / 19 / 2003 \\
1: 45: 35 \mathrm{PM}\end{array}$ & $\begin{array}{l}\text { 11/19/2003 } \\
\text { 2:00:00 PM }\end{array}$ & 0.3 & 0.3 & $\begin{array}{l}11 / 19 / 2003 \\
1: 46: 36 \text { PM }\end{array}$ \\
\hline 1233 & Price_LBNL1 & 392 & roche,cpu1_ & $\begin{array}{l}\text { 11/19/2003 } \\
1: 45: 44 \text { PM }\end{array}$ & $\begin{array}{l}\text { 11/19/2003 } \\
\text { 2:00:00 PM }\end{array}$ & 0.3 & 0.3 & $\begin{array}{l}11 / 19 / 2003 \\
1: 46: 44 \text { PM }\end{array}$ \\
\hline 1233 & Price_LBNL1 & 397 & albertsons,cpu1_ & $\begin{array}{l}11 / 19 / 2003 \\
1: 47: 05 \text { PM }\end{array}$ & $\begin{array}{l}\text { 11/19/2003 } \\
\text { 2:00:00 PM }\end{array}$ & 0.3 & 0.3 & \begin{tabular}{|l|} 
11/19/2003 \\
1:50:13 PM
\end{tabular} \\
\hline 1233 & Price_LBNL1 & 389 & gsa,cpu1_ & $\begin{array}{l}\text { 11/19/2003 } \\
2: 00: 22 \mathrm{PM}\end{array}$ & $\begin{array}{l}\text { 11/19/2003 } \\
\text { 2:15:00 PM }\end{array}$ & 0.75 & 0.75 & $\begin{array}{l}\text { 11/19/2003 } \\
2: 14: 37 \mathrm{PM}\end{array}$ \\
\hline 1233 & Price_LBNL1 & 402 & boa,cpu1_ & $\begin{array}{l}\text { 11/19/2003 } \\
\text { 2:00:38 PM }\end{array}$ & $\begin{array}{l}\text { 11/19/2003 } \\
\text { 2:15:00 PM }\end{array}$ & 0.75 & 0.75 & $\begin{array}{l}\text { 11/19/2003 } \\
\text { 2:01:40 PM }\end{array}$ \\
\hline 1233 & Price_LBNL1 & 392 & roche,cpu1_ & $\begin{array}{l}11 / 19 / 2003 \\
2: 00: 44 \mathrm{PM}\end{array}$ & $\begin{array}{l}\text { 11/19/2003 } \\
\text { 2:15:00 PM }\end{array}$ & 0.75 & 0.75 & $\begin{array}{l}11 / 19 / 2003 \\
2: 01: 44 \mathrm{PM}\end{array}$ \\
\hline 1233 & Price_LBNL1 & 385 & ucsb,cpu1_ & $\begin{array}{l}\text { 11/19/2003 } \\
\text { 2:00:50 PM }\end{array}$ & $\begin{array}{l}\text { 11/19/2003 } \\
\text { 2:15:00 PM }\end{array}$ & 0.75 & 0.75 & $\begin{array}{l}\text { 11/19/2003 } \\
\text { 2:01:50 PM }\end{array}$ \\
\hline 1233 & Price_LBNL1 & 397 & albertsons,cpu1_ & $\begin{array}{l}\text { 11/19/2003 } \\
\text { 2:02:05 PM }\end{array}$ & $\begin{array}{l}\text { 11/19/2003 } \\
\text { 2:15:00 PM }\end{array}$ & 0.75 & 0.75 & $\begin{array}{l}\text { 11/19/2003 } \\
\text { 2:05:14 PM }\end{array}$ \\
\hline
\end{tabular}

\section{4-2 Demand Shed Savings Estimates}

This section reviews the results of the second Auto-DR tests, which were more successful than the first-day test results. The first-day results are described briefly. For each site, both whole-building and component level savings estimates are discussed. For GSA, Roche, and UCSB, additional indoor environmental conditions were monitored and are discussed. 


\section{Shed Strategies and Results of Response}

Table 4-5 summarizes the shed strategies used at each site. Though some sites failed to implement some of the strategies for the first test, all the sites eventually implemented all the strategies for the second test. Albertsons disabled their anti-sweat heater shed strategy for the first test due to an electrical wiring issue. BofA didn't respond to the signal for the first test. GSA didn't poll the price signal for the first test. Roche succeeded for the both tests. The UCSB fan VFD limit strategy failed for the first test, though the cooling valve shed was successful. On the second test day, all the sites achieved all the shed strategies as planned. (See Appendix VII for more details regarding the first test).

Table 4-12. Shed Strategies and Results of Response

\begin{tabular}{|c|c|c|c|c|c|c|c|c|c|c|c|}
\hline & & \multicolumn{2}{|c|}{ Albertsons } & \multicolumn{2}{|c|}{$B$ of $A$} & \multicolumn{2}{|c|}{ GSA } & \multicolumn{2}{|c|}{ Roche } & \multicolumn{2}{|c|}{ UCSB } \\
\hline & & 1st & 2nd & $1 \mathrm{st}$ & 2nd & $1 \mathrm{st}$ & 2nd & $1 s t$ & 2nd & $1 \mathrm{st}$ & 2nd \\
\hline \multirow{4}{*}{ HVAC } & $\begin{array}{l}\text { Global zone set- } \\
\text { point increase }\end{array}$ & & & & & $d$ & 0 & & & & \\
\hline & $\begin{array}{l}\text { Direct control } \\
\text { of fans }\end{array}$ & & & & & & & 0 & 0 & $d$ & 0 \\
\hline & $\begin{array}{l}\text { Reset duct static } \\
\text { pressure }\end{array}$ & & & $d$ & 0 & & & & & O & 0 \\
\hline & $\begin{array}{l}\text { Reset cooling and } \\
\text { heating valves }\end{array}$ & & & & & & & & & 0 & 0 \\
\hline Lighting & $\begin{array}{l}\text { Reduce ambient } \\
\text { lighting }\end{array}$ & 0 & 0 & & & & & & & & \\
\hline Other & $\begin{array}{l}\text { Reduce Anti- } \\
\text { sweat Heaters }\end{array}$ & $d$ & 0 & & & & & & & & \\
\hline
\end{tabular}

\section{Saving Analysis by Site}

During the Auto-DR tests, whole building and controllable component demand were measured at all five sites. The demand savings were calculated by the savings verification methods defined in Section 3-2. This section describes procedures to develop component level baselines corresponding to each site's shed strategies. Since each building has unique shed strategies and data trending, the component level saving calculation methods are customized for each site and each component. The non-energy results are also discussed for sites where comfort measurements are available (zone temperature and/or carbon dioxide $\left(\mathrm{CO}_{2}\right)$ concentration).

\section{Albertsons}

\section{Saving Verification Methods}

Albertsons' shed strategies controlled non-HVAC components, overhead lights and antisweat door heaters. Since these components are not weather-sensitive, the componentlevel baseline method was straightforward. Fifteen-minute interval electric demands of 
WBP and the two controlled components were collected by the site's eLutions System. Baseline method for each controllable component is described below.

\section{Overhead Lights}

The equipment schedule method is used to calculate the shed values for this component. The overhead lights are 100\% on during store hours, from 6:00 am until midnight. The electric demand of the overhead lights during the store hours was constant over the two week period (average $42.7 \mathrm{~kW}$, minimum $41.8 \mathrm{~kW}$, and maximum $43.5 \mathrm{~kW}$ ). The average demand of $42.7 \mathrm{~kW}$ is used as baseline for the shedding period.

\section{Anti-sweat Door Heaters}

The anti-sweat door heaters were not well modeled with an OAT regression model. The previous days' anti-sweat door heater loads were flat during the afternoon period. An assumption could be made that these loads may also remain flat during the test period if the shed didn't occur. Thus, the baseline was estimated from the previous 15-minute load (before the first higher price signal).

\section{Saving Results}

Figure 4-1 shows, for the second day test, the whole-building method baseline and the calculated test period whole building power. Albertsons achieved maximum demand savings of $10 \%$ at $2: 45 \mathrm{pm}$ to $3: 00 \mathrm{pm}$ during the $\$ 0.75 / \mathrm{kWh}$ period.

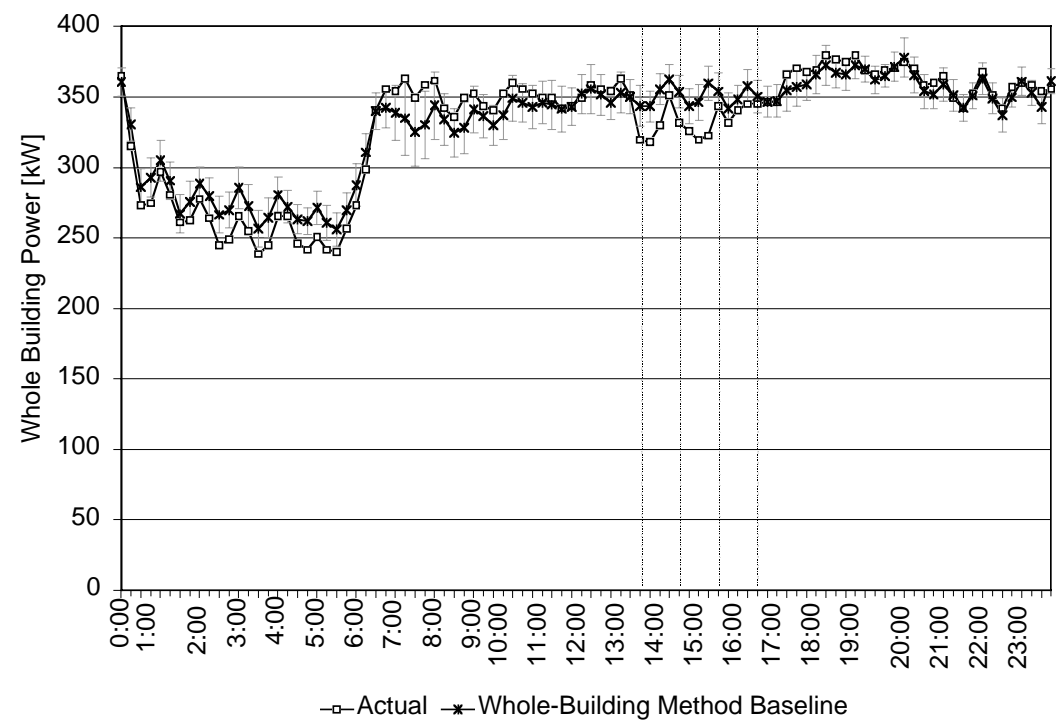

Figure 4-19. Albertsons - Whole Building Power and Baseline (Whole-Building Method)

At 1:00 pm, upon the reception of $\$ 0.30 / \mathrm{kWh}$ signal, the overhead lights were shed by the strategy. The whole building power dropped by $31 \mathrm{~kW}$. The whole building power spiked up by $21 \mathrm{~kW}$ at 1:45 pm (see Figure 4-1). The sub-meters of the controllable components measured no increase of power consumption, so this increase was due to other, non-controlled, components at the site. The whole building power dropped again by $19 \mathrm{~kW}$ at 2:00 pm, due to the anti-sweat heaters shutdown strategy activated by 
$\$ 0.75 / \mathrm{kWh}$ signal. The demand rose by $21 \mathrm{~kW}$ at 3:00 pm due to turning on the antisweat heaters at the end of $\$ 0.75 / \mathrm{kWh}$ signal. The end of the overhead lights shed couldn't be identified in the whole building power because of confounding factors in the non-controllable loads.

Figure 4-2 shows the actual demand and savings by the component method. The demand saving result of this method was slightly higher than that of the whole-building method. This is because of the demand increase in non-controllable component as mentioned earlier.

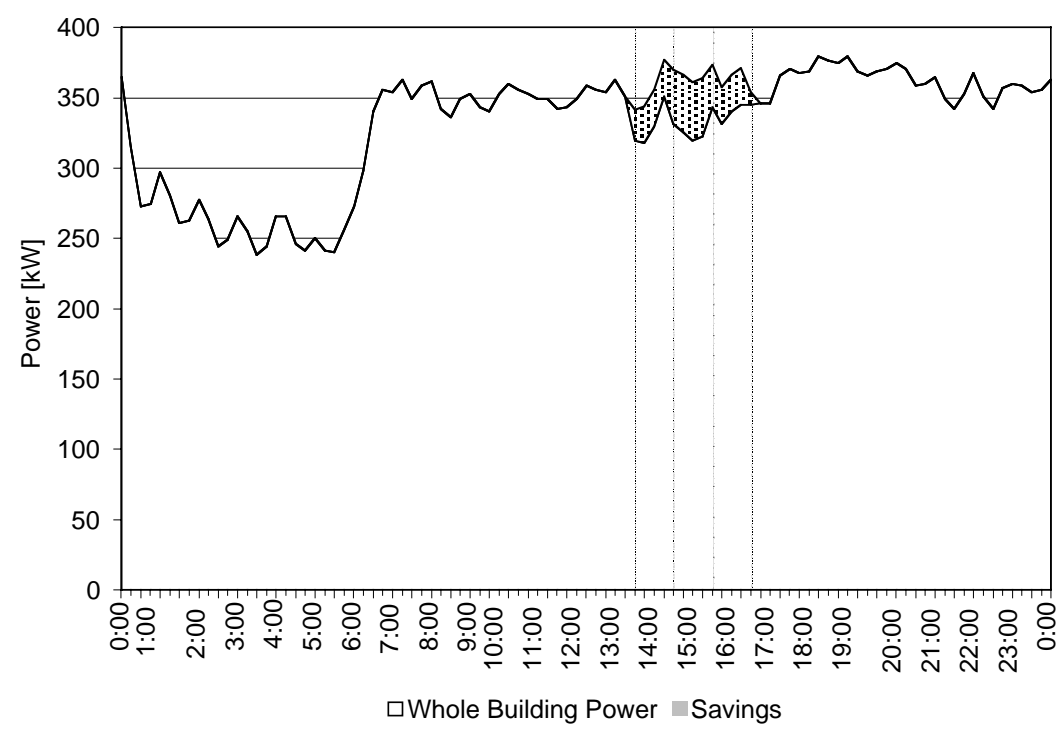

Figure 4-20. Albertsons - Whole Building Power and Savings (Component Method)

Figure 4-3 shows electric demand of the overhead lights and the anti-sweat door heaters. Both components clearly show the demand curtailment. The overhead light demand dropped by $27 \mathrm{~kW}(62 \%)$ at 1:00 pm (15 minutes before the $\$ 0.30 / \mathrm{kWh}$ time period started) and came back at 4:00 pm (15 minutes before the $\$ 0.30 / \mathrm{kWh}$ time period ended). The anti-sweat heaters demand dropped $15 \mathrm{~kW}(15 \%)$ at 2:00 pm and came back at 3:00 pm. A maximum of $42 \mathrm{~kW}$ (65\% of total controllable load) was shed from 2:00 pm to 3:00 pm. Both components reacted immediately after receiving the 15-minute ahead price signal. 


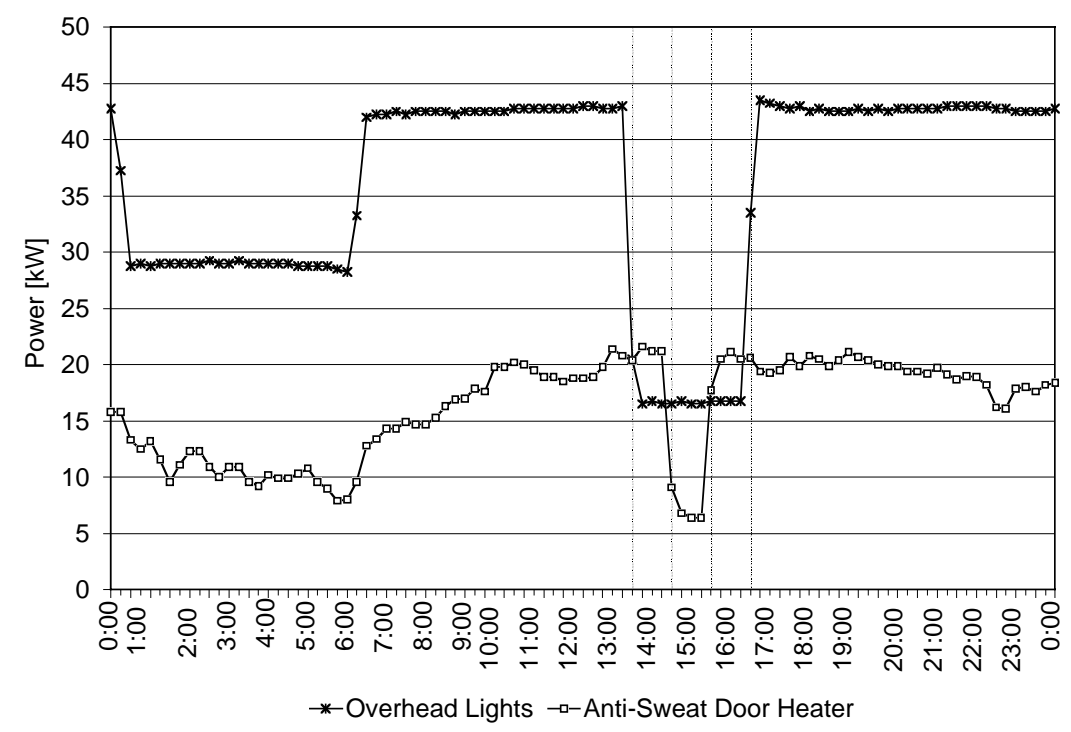

Figure 4-21. Albertsons - Overhead Lights and Anti-Sweat Door Heaters

\section{B of A}

\section{Saving Verification Methods}

As originally planned, Building B was analyzed for demand savings during the Auto-DR test. The site's EIS (WebGen) was designed to conduct sheds for the entire facility, which included three large office buildings. Duct static pressure reset was used to shed the supply and return fan demand during the test. The supply fans and the return fans of Building B were considered to be the controllable components. Looking at the measured data, the fan power was not well modeled with an OAT regression model. Therefore, the prior time load method was used for this saving analysis. The previous 15-minute demand was used as its baseline during the test period.

\section{Saving Results}

Figure 4-4 shows Building B's whole building power and whole-building method baseline. The demand shed realized was small, and no demand saving could be identified against the baseline, though there were several demand drops during the test ranging from $8 \mathrm{~kW}$ to $29 \mathrm{~kW}$. Because the WebGen system rotated the shed strategy among the three buildings to minimize the indoor comfort impacts, each building's electric demand had several short-time demand sheds during the three-hour test period. 


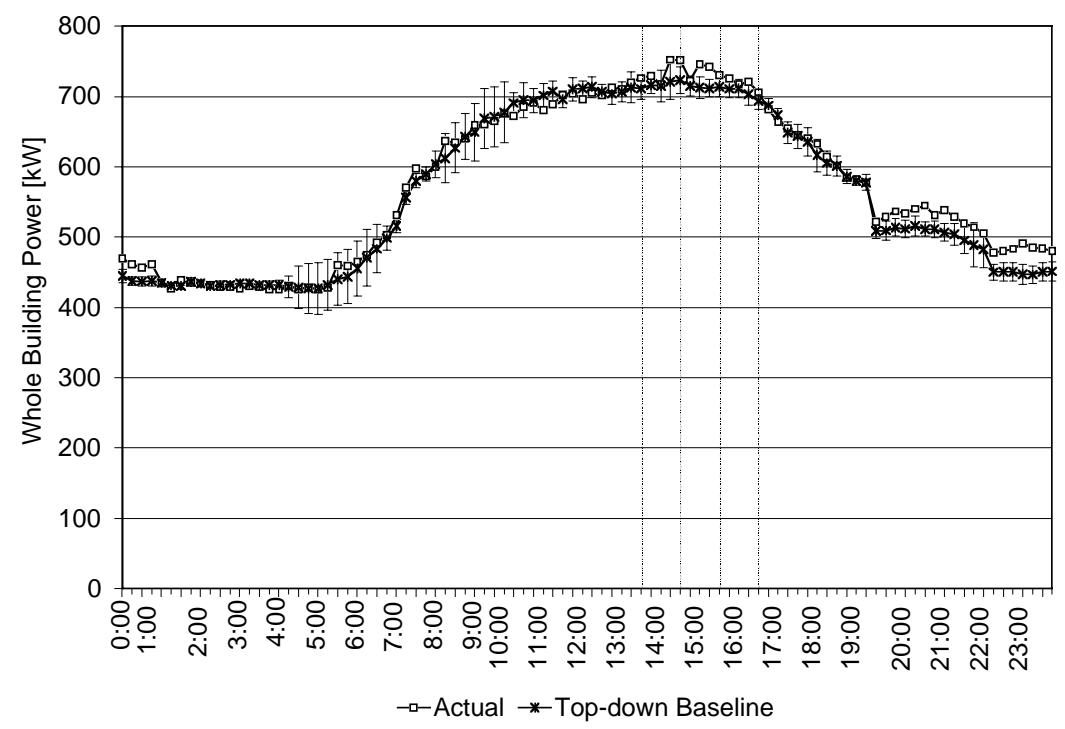

Figure 4-22. B of A - Whole Building Power and Baseline (Whole-Building Method)

Figure 4-5 shows the motor control center $(\boldsymbol{M C C})$ electric demand, which includes all of Building B's supply and return fans and motors. The fan power was reduced three times during the three-hour test period. The demand shed ranged from $7 \mathrm{~kW}$ to $11 \mathrm{~kW}$.

A WebGen analyst independently calculated the demand savings using WebGen's methodologies. The detail of the analysis is included in Appendix VII.

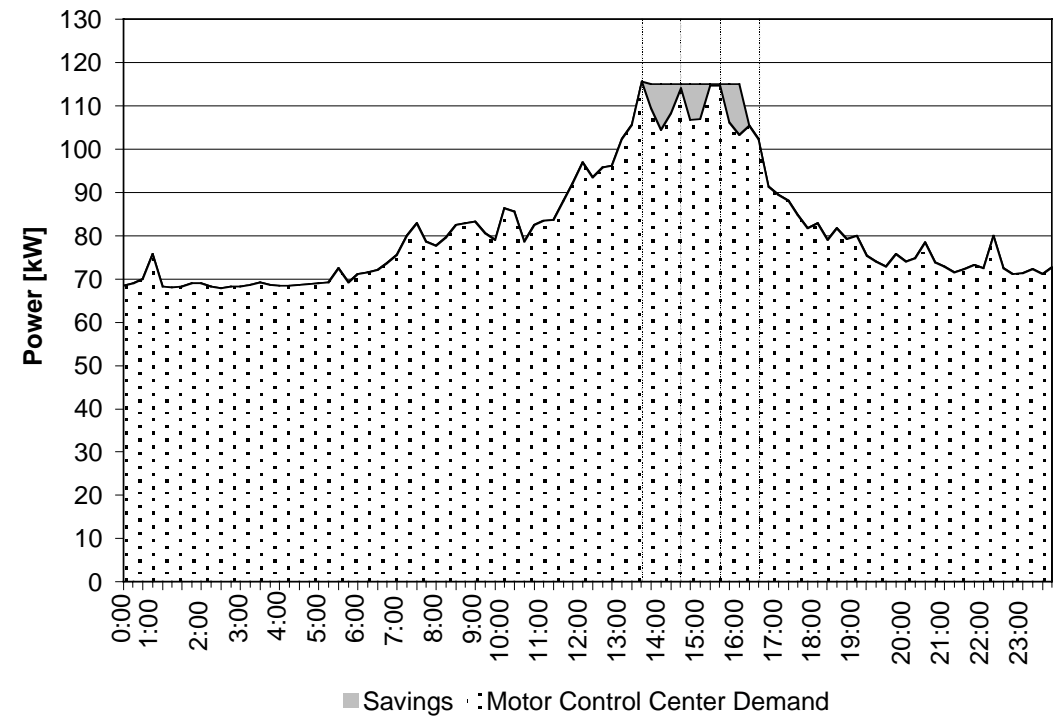

Figure 4-23. B of A - Motor Control Center

Figure 4-6 shows fan data from the WebGen Web-interface. The time-series graph shows static pressure, VFD speed, and supply air temperature. The static pressure was normally kept at 2.2 inches water column ("W.C.), but lowered to 2.0"W.C. due to the 
static pressure reset strategy. The VFD speed was slightly reduced corresponding to the static pressure reset.

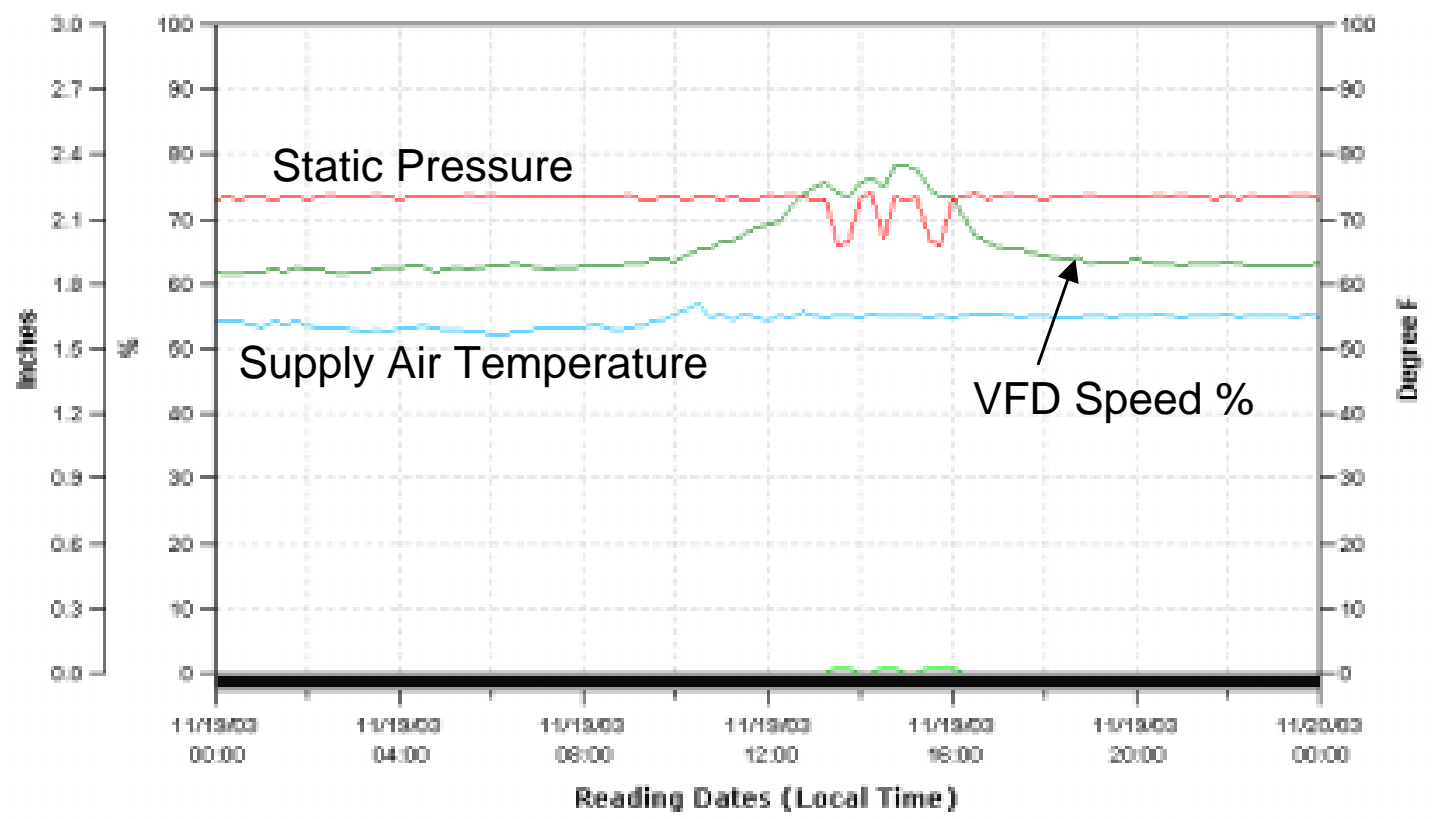

Figure 4-24. B of A - WebGen Chart

GSA

\section{Saving Verification Methods}

GSA's shed strategy implemented global zone temperature setpoint increase in cooling mode, and decrease in heating mode. The global setpoint temperature increase strategy affects most of the HVAC system end-use equipment controls. The HVAC components considered as the controllable components for this saving analysis include the chillers, primary and secondary pumps, cooling towers, and fans.

One issue impacting the analysis of the demand shed is that GSA's recently developed cooling strategy maximizes free cooling by cooling towers (tower free cooling). Because of this strategy, the chiller and the other related HVAC equipments operations provide an irregular load shape. Since this strategy has been implemented in some mornings before and during the 2-week test period, it is difficult to eliminate this irregular operational pattern from baseline input data.

\section{End-use Equipment Demand Extrapolation}

Although many of the GSA electric submeter points for their HVAC equipment were trended by the EMCS, some fan and pump submeter data necessary for the saving analysis were not directly trended by the EMCS. These values had to be extrapolated from other EMCS trend points. For constant volume systems, fan power was estimated based on equipment status and full load power from the mechanical specifications. For 
variable volume systems, fan power was estimated from the VFD speed and scheduled power $^{10}$.

\section{End-use Equipment Demand Baseline}

The OAT regression model method was used to develop a baseline for each controllable component for the saving analysis. Though internal loads mostly dominated the cooling loads in this building, we found that each component power has correlation against OAT. Individual baseline models were developed for the chillers, pumps, cooling towers, and fans respectively. This analysis is based on an assumption that the irregular patterns caused by the tower free cooling strategy would be averaged out by the model.

\section{Saving Results}

Figure 4-7 shows GSA's whole building power and the whole-building method baseline. Due to the tower free cooling operation in the morning, the chillers were off in the early morning, and turned on around 11:30 am. The whole building power peaked at 11:45 am because of the chiller startup. The global temperature setpoint increase (from 72 to 76 ${ }^{\circ} \mathrm{F}$ ) was implemented at 1:00 pm responding to the 15 -minute ahead $\$ 0.30 / \mathrm{kWh}$ price signal. The whole building power dropped by $80 \mathrm{~kW}$ by $1: 15 \mathrm{pm}$, and further dropped by $75 \mathrm{~kW}$ at 1:30 pm. Using the whole-building method, the maximum demand saving was $274 \mathrm{~kW}$ at 2:00 pm, which is $12 \%$ of whole building power. However, because the baseline includes the intermittent impact of the tower free cooling strategy, the baseline can have a wide error range.

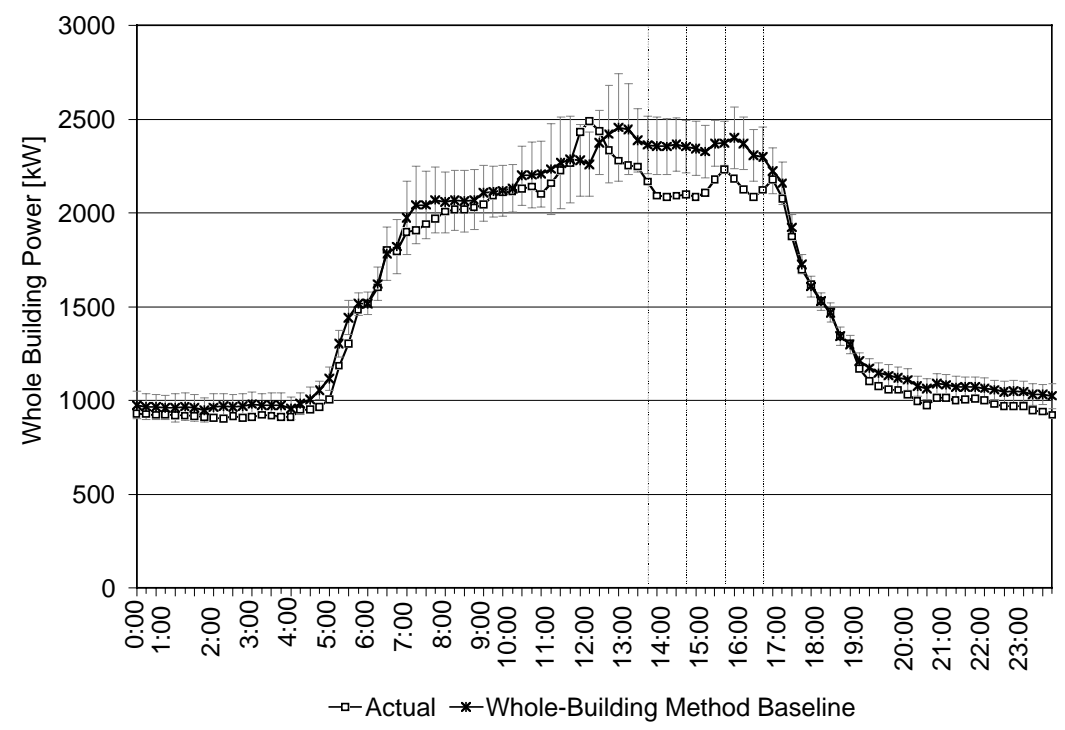

Figure 4-25. GSA - Whole Building Power and Baseline (Whole-Building Method)

Figure 4-8 shows whole building power, HVAC demand and savings by the component method. The OAT regression model was developed for each controllable component to estimate the savings. All of the component savings are summed. (See Appendix VII for

\footnotetext{
${ }^{10}$ The equation used to calculate electric demand is: equipment demand $[\mathrm{kW}]=$ scheduled power $[\mathrm{kW}] \mathrm{x}$ $(\text { VFD Speed }[\%])^{2}$. Some adjustment factors are also applied based on comparisons of the calculated values to the submetered data.
} 
the actual demand and OAT regression model graph of each controllable component). The maximum savings in whole building power by the component method was $240 \mathrm{~kW}$ $(10 \%)$ at $3: 45 \mathrm{pm}$.

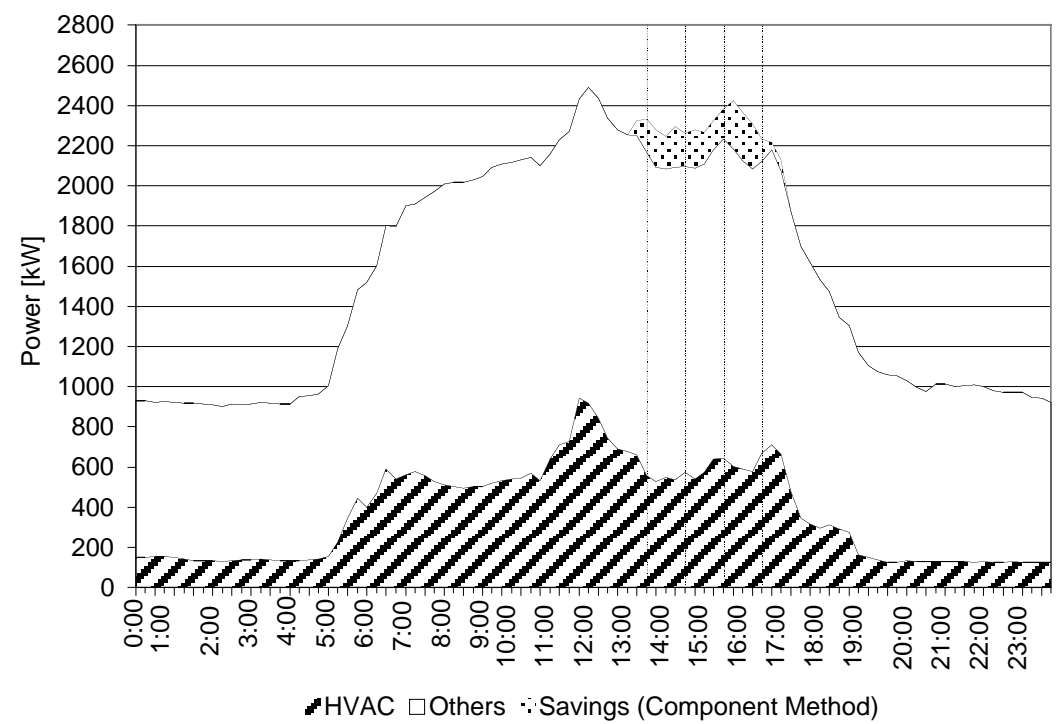

Figure 4-26. GSA - HVAC Demand and Savings (Component Method)

Figure 4-9 shows the electrical demand of each HVAC component. The fan power showed the largest demand savings in the HVAC components. The fan controls responded quickly to the price signal setting. The fan demand dropped by $71 \mathrm{~kW}$ at 1:00 $\mathrm{pm}$ and increased by $72 \mathrm{~kW}$ at 4:00 pm. The maximum demand saving of the fan power was $138 \mathrm{~kW}$ at 3:45 pm.

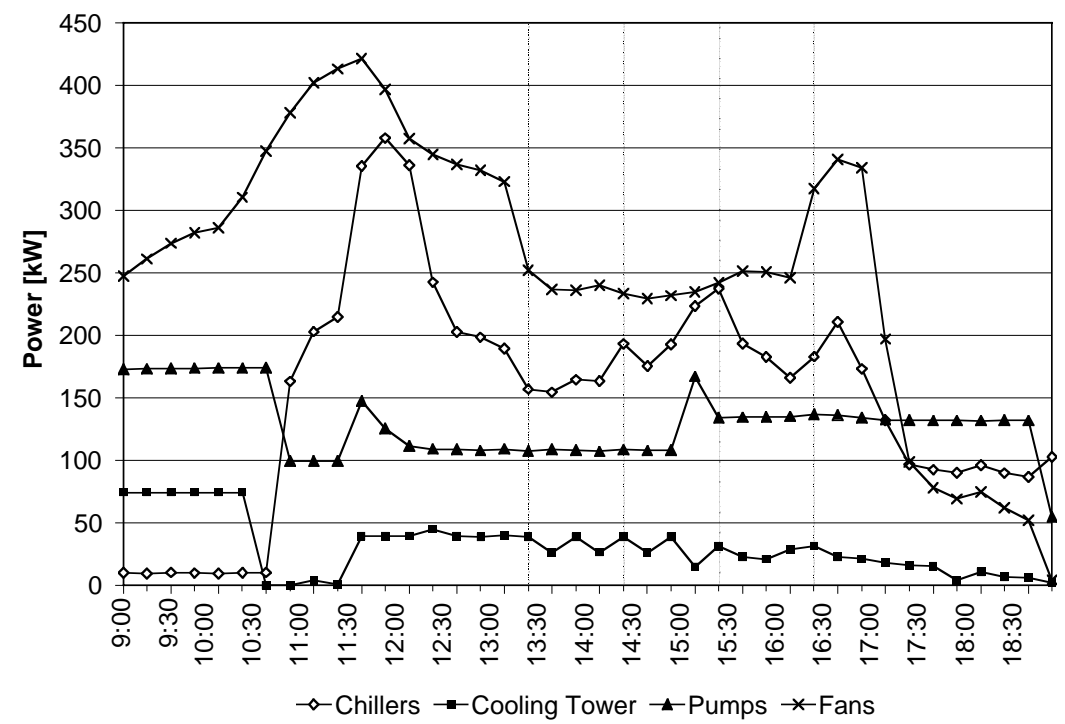

Figure 4-27. GSA - HVAC Component Demand

When the first $\$ 0.30 / \mathrm{kWh}$ signal was received $(1: 00 \mathrm{pm})$, the setpoint increased from 72 to $76^{\circ} \mathrm{F}$. Since the zone temperature setpoints were kept at $72^{\circ} \mathrm{F}$ before the test started, 
the zones were "pre-cooled" compared to the $76^{\circ} \mathrm{F}$ setpoint. The chiller power was reduced by approximately $40 \mathrm{~kW}$ until the zone temperatures rose to $76^{\circ} \mathrm{F}$. The chiller demand crept up little by little, as some zones were reaching $76^{\circ} \mathrm{F}$. By $2: 15 \mathrm{pm}$, the chiller demand increased, probably because many zones reached $76^{\circ} \mathrm{F}$ and required cooling. Right after the chiller demand rise, the $\$ 0.75 / \mathrm{kWh}$ signal kicked in which increased the setpoint to $78^{\circ} \mathrm{F}$. The chiller demand dropped again. However, the zone temperatures soon reached the new setpoint and chiller demand rose again. At 3:00 pm, the chiller demand further increased by the second $\$ 0.30 / \mathrm{kWh}$ signal which decreased the setpoint back to $76^{\circ} \mathrm{F}$. However, the chiller demand suddenly decreased after 3:15 pm. This may have been caused by a factor irrelevant to the shed strategy. The chiller demand increased again at 4:00 pm. when the setpoint decreased to $72^{\circ} \mathrm{F}$ at the end of the test.

The pump electrical demand total contains the condenser water pumps, the primary chilled water pumps and the secondary chilled water pumps (See Appendix II for the pump demands on the test day). The pump demand increased to $167 \mathrm{~kW}$ at 2:45 pm responding to staging-up of the chiller operation, and stayed at $130 \mathrm{~kW}$ until the end of the day's scheduled operation. Because the pumps support the chiller operation, pump demand did not respond to the price signal promptly. The cooling tower demand fluctuated significantly during the test period, and remarkable demand saving could not be determined.

\section{Non-Energy Issues}

Zone temperatures for 39 zones ( 25 zones from 6 floors in North Tower, and 14 zones from 3 floors in South Tower) were trended and analyzed at GSA. VAV damper positions ( 19 zones) were also collected. Figure $4-10$ shows the average of the 39 zone temperatures during the test day, overlaid on the average zone temperature based on the previous 18 days. (See Appendix VII for time-series charts of the zone temperatures and VAV damper positions for each floor). During normal building operation, the average zone temperature was about $72{ }^{\circ} \mathrm{F}$. During the shed, the average zone temperature rose by $1{ }^{\circ} \mathrm{F}\left(72{ }^{\circ} \mathrm{F}\right.$ to $\left.73^{\circ} \mathrm{F}\right)$, although the shed strategy allowed the global temperature setpoint to increase to $76^{\circ} \mathrm{F}$ at $\$ 0.30 / \mathrm{kWh}$, and $78^{\circ} \mathrm{F}$ at $\$ 0.75 / \mathrm{kWh}$. The average zone temperature dropped to below $72{ }^{\circ} \mathrm{F}$ after the shed ended. 


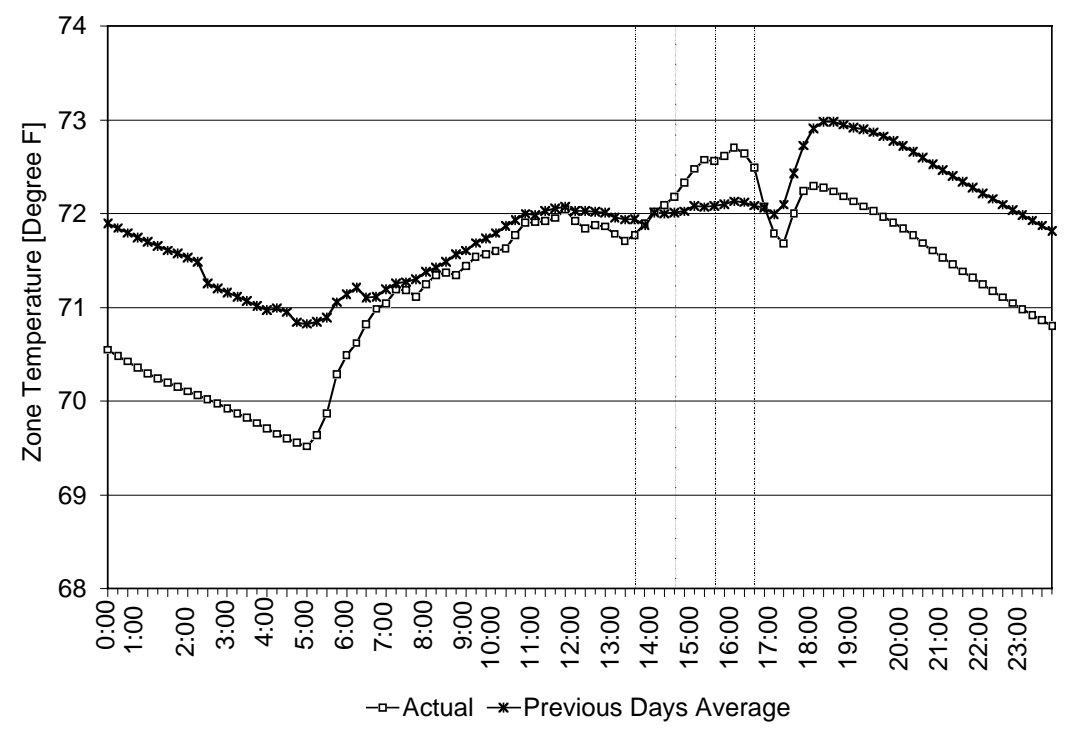

Figure 4-28. GSA - Zone Temperature

Individual zone temperature were also analyzed (see Appendix II). The controls for some zones responded to the global temperature setpoint strategy, with some zone temperatures increasing closer to or above $76^{\circ} \mathrm{F}$ at the $\$ 0.30 / \mathrm{kWh}$ signal, and $78^{\circ} \mathrm{F}$ at the $\$ 0.75 / \mathrm{kWh}$ signal. However, most of the zone temperatures were not affected at all. Most VAV boxes (17 of 19 collected) didn't change their damper positions during the test period. This may be because 1) the damper positions were less than $20 \%$ for most of VAV boxes which may be the minimum damper position allowed by the system in order to maintain minimum ventilation rates; and 2) the temperature difference might be within the control's deadband.

Although most of the monitored zones didn't respond to the setpoint increase strategy, some zones indicated temperature changes and the HVAC component electric data indicated demand savings due to the strategy. Since zone temperature and VAV damper position data was collected for a small subset of the zones, it is hard to determine what happened in the other zones.

\section{Roche}

\section{Saving Verification Methods}

Three Roche buildings, including A2 (office, auditorium), SS (office), and FS (cafeteria) participated in the Auto-DR test. Some of the fans in each building were turned off based on the price signal. Since all the fans are constant volume, the fan electric demand was extrapolated from the spot measurement data and normal operation schedule. Since the building's cooling loads are supplied from a cooling plant that serves multiple buildings, only the fan power is counted as a controllable load. We did not evaluate whether there was any reduction in the cooling loads supplied to the buildings from the central plant. 


\section{Saving Results}

Figure 4-11 shows the whole building power and the whole-building method baseline applied to all three buildings. The power measurements for each building and the wholebuilding baseline models are shown in Appendix VII. Building FS (cafeteria) electric demand had several spikes in the morning because of food preparation. Building FS demand declined rapidly after 1:00 pm because occupants left after lunchtime. Building SS changed its equipment operation on October $30^{\text {th }}$, and reduced approximately $50 \mathrm{~kW}$ of its demand. The whole-building method may project higher demand savings than expected, because the whole-building method baseline was affected by the demand profile change of Building SS.

The whole-building analysis (including all the three buildings) indicates that Roche achieved maximum $164 \mathrm{~kW}$ (28\%) of demand savings from 2:45 pm to 3:00 pm. The levels of demand shed due to the different price signals can be clearly seen in the demand profile. The whole building power dropped by $71 \mathrm{~kW}$ at $1: 00 \mathrm{pm}$ at the first $\$ 0.30 / \mathrm{kWh}$ signal due to the implementation of the fan shed strategy. The demand dropped further by $57 \mathrm{~kW}$ at $2: 00 \mathrm{pm}$ at the $\$ 0.75 / \mathrm{kWh}$ signal.

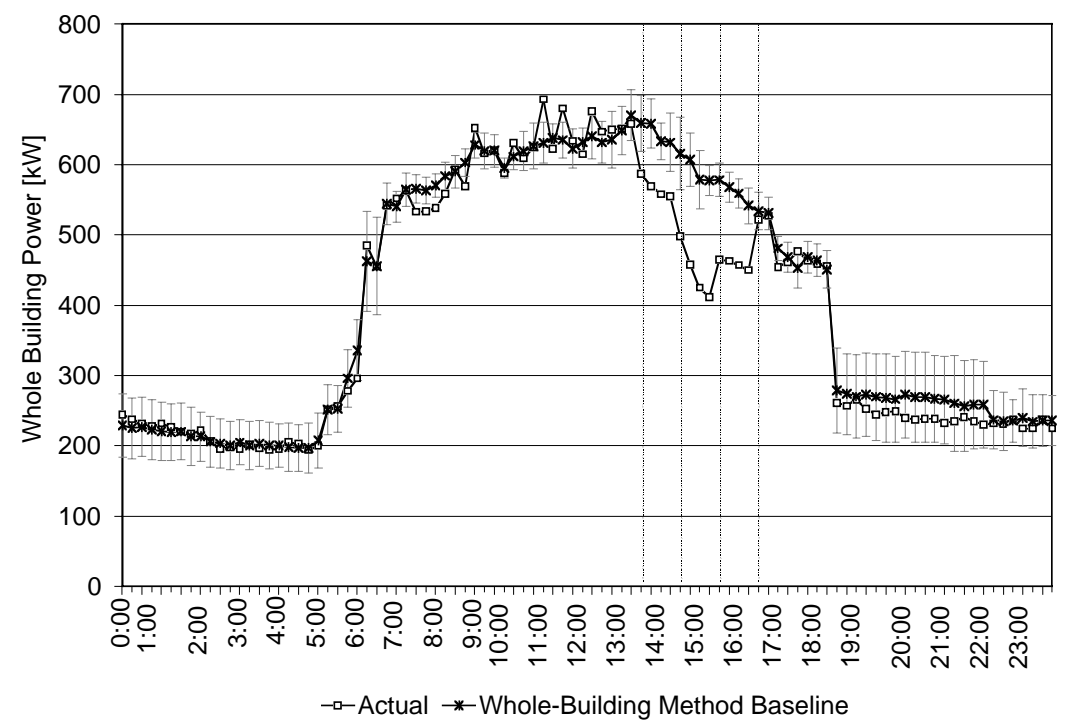

Figure 4-29. Roche - Combined Whole Building Power and Baseline (Whole-Building Method applied to all Three Buildings)

Figure 4-12 shows whole building power, fan power and savings by the component method. The whole building power was reduced by $70 \mathrm{~kW}$ due to the air handler shedding at Building A2 activated at the $\$ 0.30 / \mathrm{kWh}$ signal. It was further reduced by 37 $\mathrm{kW}$ by Building SS and FS fan shedding at the $\$ 0.75 / \mathrm{kWh}$ signal. The maximum shed of all three buildings was $107 \mathrm{~kW}$. This site collected 5-minute interval data for the fan status. The status data verified that all the selected air handlers followed the price signal within five minutes after receiving the new price signal $(1: 00 \mathrm{pm}, 2: 00 \mathrm{pm}, 3: 00 \mathrm{pm}$, and 4:00 pm). 


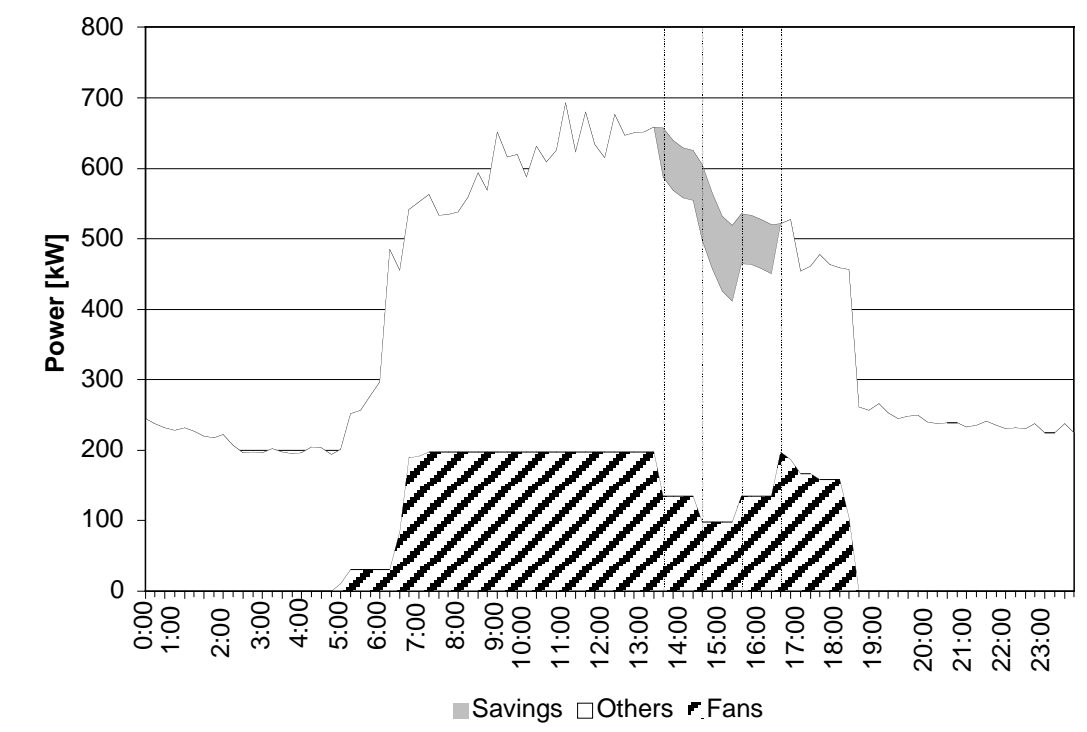

Figure 4-30. Roche - Whole Building, Fan Power and Savings (Component Method)

\section{Non-Energy Results}

Zone temperature and $\mathrm{CO}_{2}$ concentration data was collected for Building A2. Figure 413 shows Building A2's average zone temperature on the test day (November $19^{\mathrm{th}}$ ) and a reference day (November $18^{\text {th }}$ ). The zone temperature was around $74^{\circ} \mathrm{F}$ after 11:00 am, and persisted until the end of the day's occupied period. There were no temperature or airflow complaints.

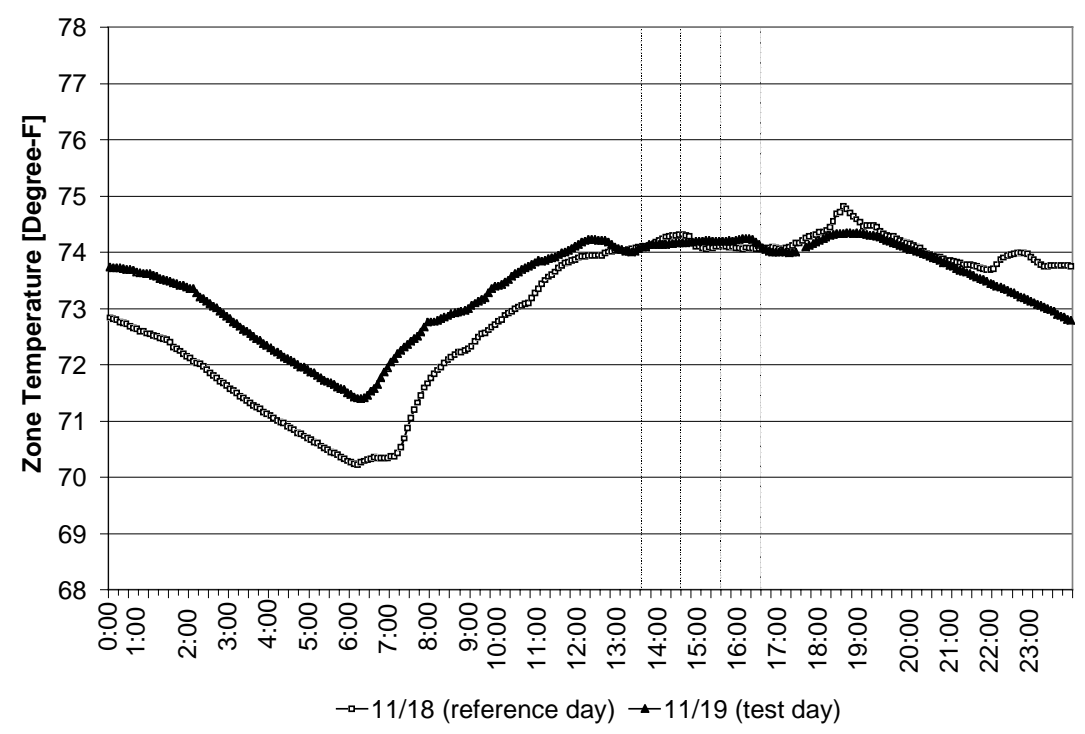

Figure 4-31. Roche - Building A2, Zone Temperature

Figure 4-14 shows Building A2's $\mathrm{CO}_{2}$ concentrations on the test day (November $19^{\text {th }}$ ) and a reference day (November $18^{\text {th }}$ ). The Building's $\mathrm{CO}_{2}$ level was quite different between the test day and the reference day, possible due to changes in the number of 
occupants. Based on the data available, it is difficult to correlate the $\mathrm{CO}_{2}$ changes during the shed period to the fan shed strategy. However, the $\mathrm{CO}_{2}$ concentration immediately decreased at the end of the test $(4: 00 \mathrm{pm})$. This was caused by turning back on the air handlers, which were off during the DR test. During the test period, the $\mathrm{CO}_{2}$ concentration was lower than $500 \mathrm{ppm}^{11}$, and there were no indoor air quality complaints.

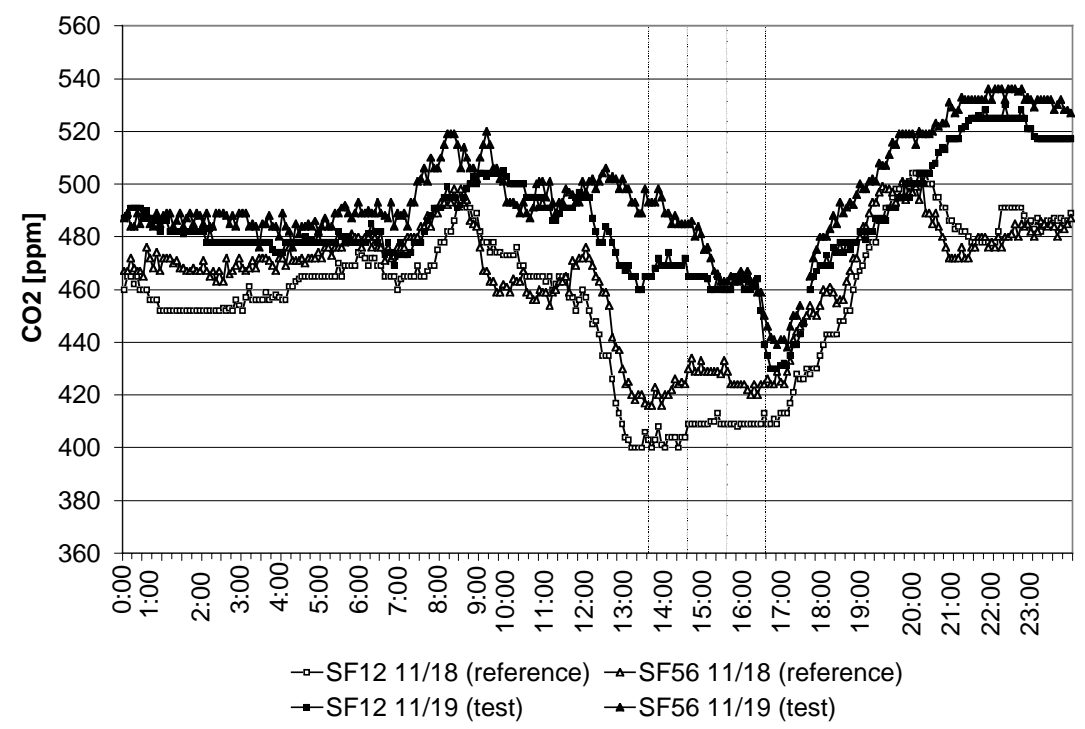

Figure 4-32. Roche - Building $\mathrm{A} 2, \mathrm{CO}_{2}$ Concentration

\section{UCSB}

\section{Saving Verification Methods}

UCSB implemented the Auto-DR test at their Main Library, three inter-connected buildings (Library II, III and IV). Since the energy manager occasionally implemented some energy saving strategies at the buildings, the days he manually implemented energy saving strategies are eliminated from the baseline input data. Those manually controlled days are October $15^{\text {th }}, 16^{\text {th }}$ and $23^{\text {rd }}$. All the data used in the saving analysis were collected by the EIS. Prior to the test period, only whole building power and cooling power data were trended. For the component level evaluation, new data points were added to the EIS trend derived from the EMCS (See Table 3-7). Several zone temperatures were added to the data trend to evaluate the zone conditions. The supply fan and cooling power are considered as the controllable components.

\section{Supply Fans}

The VFD percent limit shed strategies were applied to Library II Supply Fan \#1 through \#5 and Library III Supply Fan \#2. According to the average previous-day profile, the Library II supply fan demands were flat during afternoon. Though the OAT regression

11 Comfort criteria, with respect to human bioeffluents (odor) are likely to be satisfied if the ventilation results in indoor $\mathrm{CO}_{2}$ concentrations less than 700 ppm above the outdoor air concentration (ASHRAE 622001). 
model method was tried for these fans, the actual fan load was higher than the maximum range of standard error of the model. Assuming the afternoon fan load was flat, the baseline demands for the Library II supply fans were estimated from the 15-minute demand prior to the shed. The Library III Supply Fan \#2 demand was not constant and it didn't respond to the shed strategy, so the OAT regression model was used to estimate the Library III Supply Fan \#2 demand.

\section{Cooling Power}

Though the Library has a central chiller plant, it is separated from the whole building power meter because the chiller plant supplies chilled water to a campus chilled water loop. The cooling energy [Btu/h] supplied to the Library is converted to electric demand $[\mathrm{kW}]^{12}$. Baseline cooling power was estimated by OAT regression model.

\section{Saving Results}

Figure 4-15 shows the whole building power of the Library and the whole-building method baseline. Based on the whole-building method analysis, the Library achieved a maximum $109 \mathrm{~kW}(14 \%)$ demand savings at 2:15 pm and 2:45 pm, during the $\$ 0.75 / \mathrm{kWh}$ period. The whole building power dropped by $48 \mathrm{~kW}$ at 1:00 pm as a result of the supply fan VFD shed strategy. The whole building power further dropped by $86 \mathrm{~kW}$ at 2:00 pm due to the deeper shed of supply fan VFD, static pressure limit, and cooling valve shutdown. The whole building power spiked at 3:00 pm. This spike was caused by the release of the cooling valve.

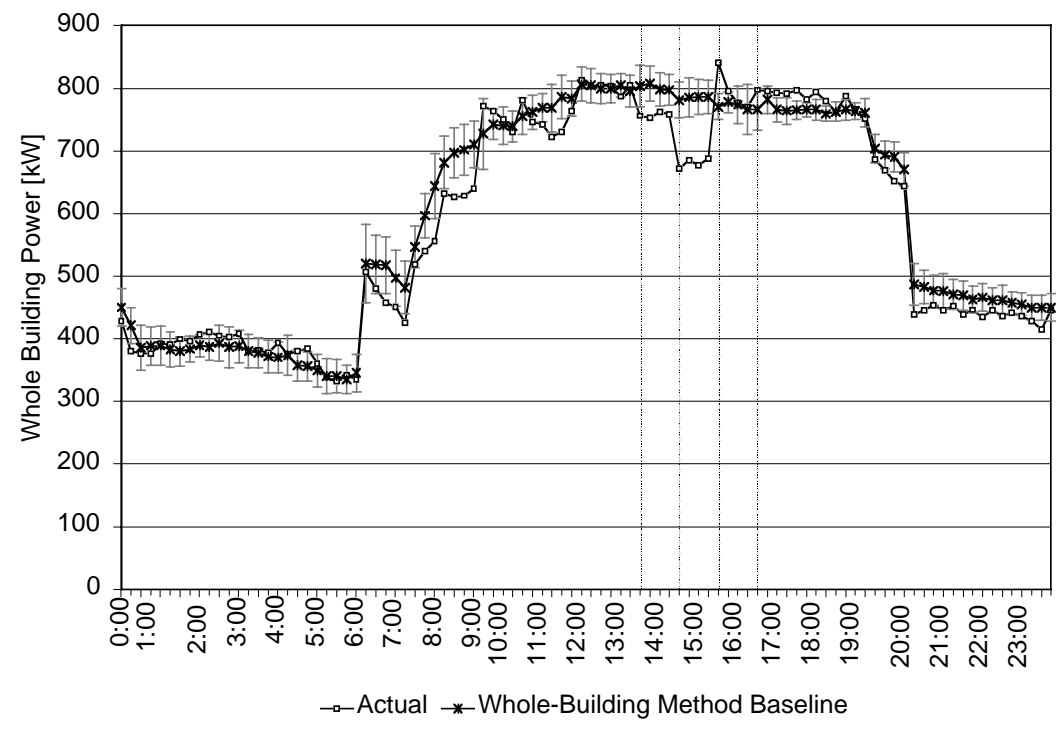

Figure 4-33. UCSB - Whole Building Power and Baseline (Whole-Building Method)

Table 4-7 shows the breakdown of whole building power and estimated savings by the component method. The maximum whole building power savings was $240 \mathrm{~kW}(10 \%)$ at

\footnotetext{
${ }^{12}$ Library cooling power $[k W]=$ central plant power $[k W] x$ Library cooling energy use $[$ Btu/h] / total cooling energy produced [Btu/h]
} 
3:45 pm, calculated using the component method. (See Appendix VII for OAT regression model baselines for each component)

Table 4-13. UCSB -Estimated Savings by Component (Component Method)

\begin{tabular}{|c|c|c|c|c|c|c|c|c|c|c|c|c|c|c|c|c|c|}
\hline \multirow{2}{*}{\begin{tabular}{|l|} 
Time \\
Price signal \\
\end{tabular}} & $\begin{array}{r}\text { from } \\
\text { to }\end{array}$ & \begin{tabular}{|l|}
$12: 45$ \\
$13: 00$ \\
\end{tabular} & \begin{tabular}{|l|}
$13: 00$ \\
$13: 15$ \\
\end{tabular} & \begin{tabular}{|l|}
$13: 15$ \\
$13: 30$ \\
\end{tabular} & \begin{tabular}{|l|}
$13: 30$ \\
$13: 45$ \\
\end{tabular} & \begin{tabular}{|l|}
$13: 45$ \\
$14: 00$ \\
\end{tabular} & \begin{tabular}{l|l}
$14: 00$ \\
$14: 15$ \\
\end{tabular} & \begin{tabular}{|l|}
$14: 15$ \\
$14: 30$ \\
\end{tabular} & \begin{tabular}{|l|}
$14: 30$ \\
$14: 45$ \\
\end{tabular} & \begin{tabular}{|l|}
$14: 45$ \\
$15: 00$ \\
\end{tabular} & $\begin{array}{l}15: 00 \\
15: 15 \\
\end{array}$ & $\begin{array}{l}15: 15 \\
15: 30 \\
\end{array}$ & \begin{tabular}{|l|}
$15: 30$ \\
$15: 45$ \\
\end{tabular} & \begin{tabular}{|l|}
$15: 45$ \\
$16: 00$ \\
\end{tabular} & $\begin{array}{l}16: 00 \\
16: 15 \\
\end{array}$ & \begin{tabular}{|l|}
$16: 15$ \\
$16: 30$ \\
\end{tabular} & $\begin{array}{l}16: 30 \\
16: 45 \\
\end{array}$ \\
\hline & $\$ / \mathrm{kW}$ & \multicolumn{2}{|c|}{0.10} & \multicolumn{4}{|c|}{0.30} & \multicolumn{4}{|c|}{0.75} & \multicolumn{4}{|c|}{0.30} & \multicolumn{2}{|c|}{0.10} \\
\hline \multirow[t]{3}{*}{ Actual } & WBP & 804 & 756 & 753 & 763 & 758 & 672 & 685 & 677 & 688 & 841 & 795 & 777 & 770 & 798 & 796 & 793 \\
\hline & Supply Fans & 120 & 94 & 94 & 101 & 98 & 77 & 76 & 79 & 81 & 98 & 97 & 95 & 94 & 116 & 116 & 115 \\
\hline & Cooling Power & 122 & 108 & 100 & 103 & 86 & 40 & 21 & 27 & 25 & 147 & 118 & 103 & 99 & 114 & 109 & 111 \\
\hline \multirow[t]{3}{*}{ Baseline } & WBP & 802 & 794 & 803 & 802 & 813 & 789 & 814 & 800 & 807 & 822 & 797 & 791 & 788 & 778 & 793 & 776 \\
\hline & Supply & 120 & 119 & 118 & 124 & 122 & 121 & 120 & 122 & 125 & 122 & 121 & 119 & 118 & 116 & 116 & 115 \\
\hline & Cooling Power & 120 & 121 & 127 & 119 & 117 & 113 & 106 & 106 & 100 & 104 & 95 & 94 & 92 & 94 & 106 & 94 \\
\hline \multirow[t]{3}{*}{ Shed kW } & WBP & -2 & 37 & 51 & 40 & 55 & 117 & 129 & 123 & 119 & -19 & 1 & 15 & 17 & $\overline{-19}$ & -3 & -17 \\
\hline & Supply Fans & 0 & 24 & 24 & 24 & 24 & 44 & 44 & 44 & 44 & 24 & 24 & 24 & 25 & 0 & 0 & \\
\hline & Cooling Power & -2 & 13 & 27 & 16 & 31 & 73 & 85 & 80 & 75 & -43 & -23 & -9 & -7 & -20 & -3 & -17 \\
\hline \multirow[t]{3}{*}{ Shed \% } & WBP & $0 \%$ & $5 \%$ & $6 \%$ & $5 \%$ & $7 \%$ & $15 \%$ & $16 \%$ & $15 \%$ & $15 \%$ & $-2 \%$ & $0 \%$ & $2 \%$ & $2 \%$ & $-2 \%$ & $0 \%$ & $-2 \%$ \\
\hline & Supply Fans & $0 \%$ & $20 \%$ & $21 \%$ & $19 \%$ & $20 \%$ & $36 \%$ & $37 \%$ & $36 \%$ & $35 \%$ & $20 \%$ & $20 \%$ & $20 \%$ & $21 \%$ & $0 \%$ & $0 \%$ & $0 \%$ \\
\hline & Cooling Power & $-2 \%$ & $11 \%$ & $21 \%$ & $13 \%$ & $26 \%$ & $65 \%$ & $80 \%$ & $75 \%$ & $75 \%$ & $-42 \%$ & $-24 \%$ & $-10 \%$ & $-8 \%$ & $-21 \%$ & $-3 \%$ & $-18 \%$ \\
\hline
\end{tabular}

Figure 4-16 shows the whole building power, the cooling power and savings by the component method. The cooling demand was affected directly by the cooling valve shutdown strategy ( $\$ 0.75 / \mathrm{kWh}$ signal), and indirectly by the fan VFD reduction strategy ( $\$ 0.30 / \mathrm{kWh}$ and $\$ 0.75 / \mathrm{kWh}$ signal). The cooling power dropped by $20 \mathrm{~kW}$ at the first $\$ 0.30 / \mathrm{kWh}$ signal. The cooling power further dropped by $80 \mathrm{~kW}$ at the $\$ 0.75 / \mathrm{kWh}$ signal. At the end of the $\$ 0.75 / \mathrm{kWh}$ signal, the cooling power increased by $120 \mathrm{~kW}$, more than $40 \mathrm{~kW}$ over the baseline. This was caused by the release of the cooling valve. Because the cooling valve control responded immediately after reception of 15-minute ahead price signal, the cooling power increased at 3:00 pm, while the price changed from $\$ 0.75 / \mathrm{kWh}$ to $\$ 0.30 / \mathrm{kWh}$ at $3: 15 \mathrm{pm}$. This immediate response may result in higher energy costs if a demand spike occurs while the price is still high. Also, the demand spike caused by the release of shed strategies may result in higher demand charges.

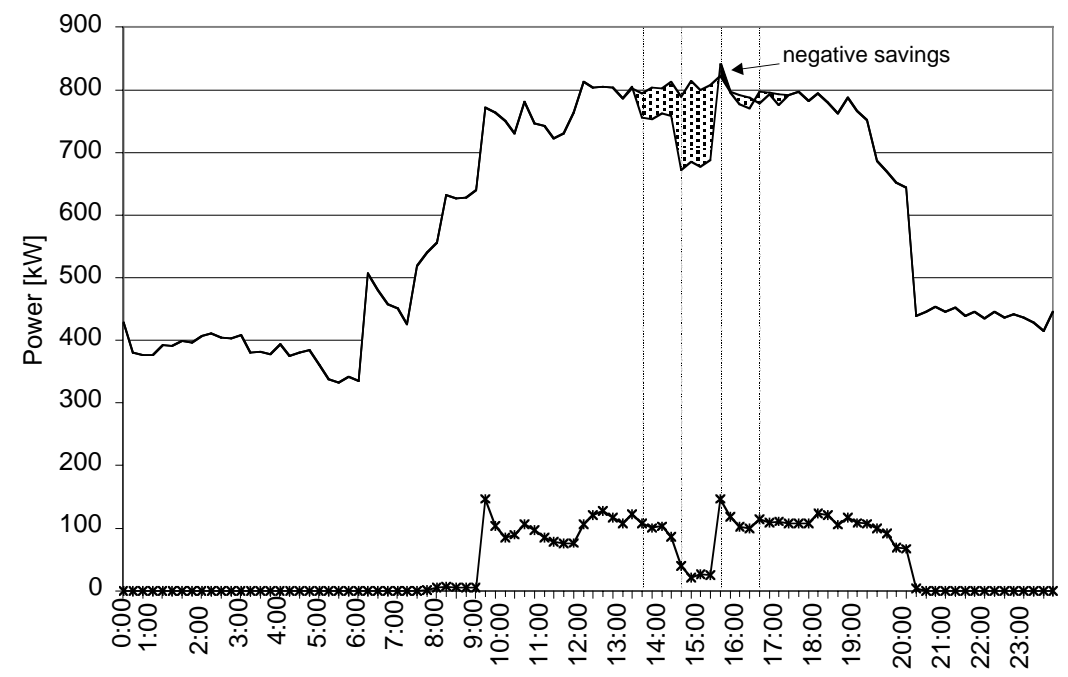

$\square$ WBP $\square$ Savings *-Cooling Power

Figure 4-34. UCSB - Whole Building Power, Cooling Energy and Savings (Component Method) 
Figure 4-17 shows the total fan power and baseline. The total fan demand dropped by 24 $\mathrm{kW}$ at 1:00 pm upon the receipt of the $\$ 0.30 / \mathrm{kWh}$ signal which initiated the VFD percentage limit strategy. The total fan demand achieved $43 \mathrm{~kW}$ saving during the $\$ 0.75 / \mathrm{kWh}$ period.

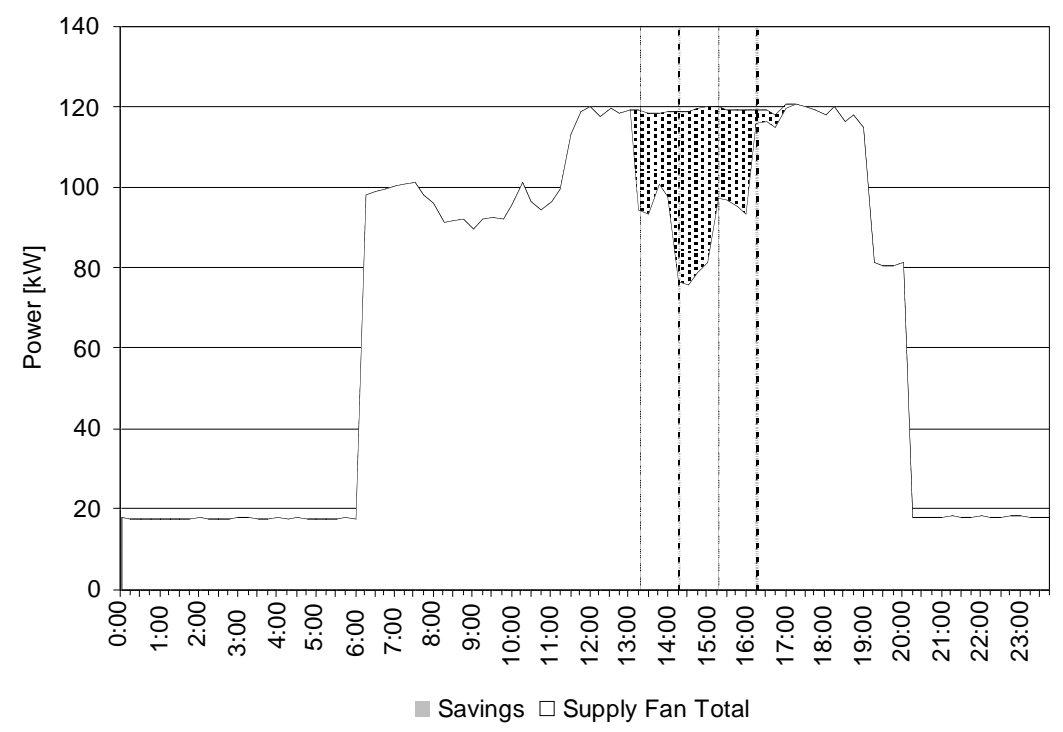

Figure 4-35. UCSB - Fan Power and Savings

\section{Non-Energy Issues}

Twenty-one zone temperatures were trended at the Library. Figure 4-18 shows the Library's average zone temperature on the test day and the reference day (average of previous 15 days). Zone temperatures of each building are given in Appendix VII. Although zone temperature increases were anticipated due to the shed strategy, average zone temperature changes were within $1^{\circ} \mathrm{F}$ during the test. The building's has a large thermal mass, due to the building mass and books, so the zone temperature change might be moderated due to the thermal mass effect. There were no occupant complaints during the test. 


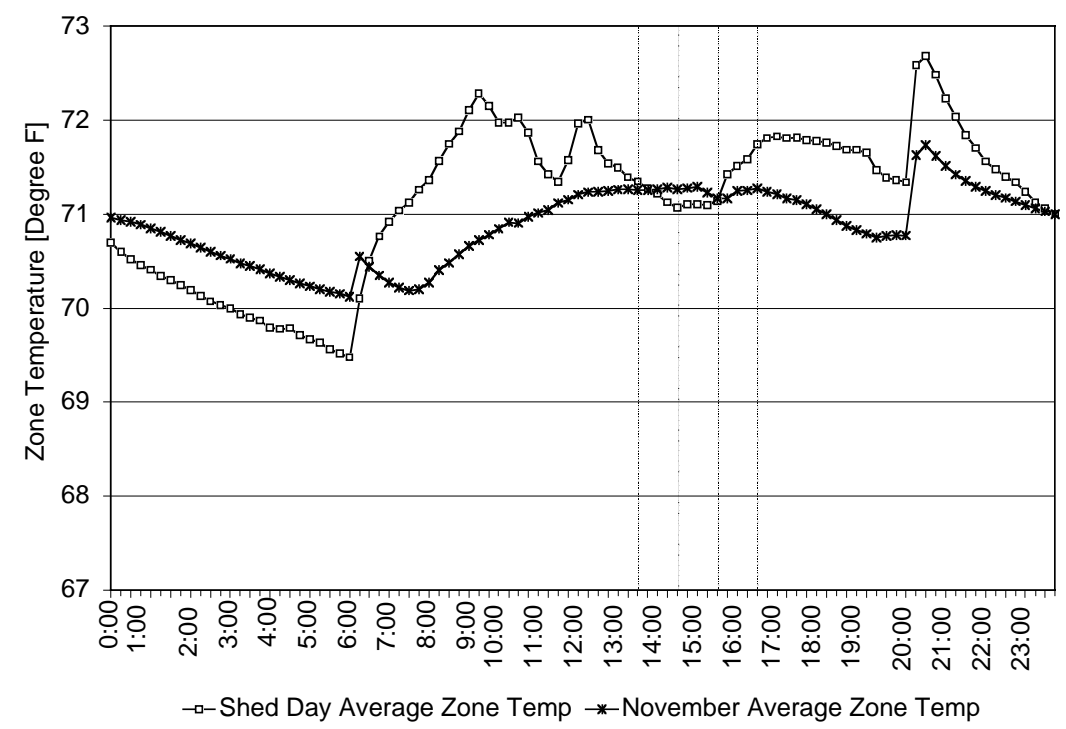

Figure 4-36. UCSB - Average Zone Temperature

\section{Summary of Results}

This section summarizes the results of the second test (November $19^{\text {th }}$ ). The demand savings are presented as shed $\mathrm{kW}$, shed $\%$, and shed $\mathrm{kW}$ per square feet $\left(\mathrm{kW} / \mathrm{ft}^{2}\right)$. Shed $\mathrm{kW}$ is calculated by subtracting the actual whole building power from its baseline demand. Shed percentage is defined as the percentage of savings in whole building power. Shed $\mathrm{kW} / \mathrm{ft}^{2}$ is the demand shed intensity, defined as the shed $\mathrm{kW}$ normalized by the building conditioned floor area.

Table 4-7 shows the demand savings for each 15 -minute period which were derived using the whole-building method. Table 4-8 shows the results summary for all sites by the component method. The aggregated shed demand calculated using the component method was similar to the results calculated using the whole-building method.

Based on the whole-building method, the maximum aggregated shed demand of all the five sites was $519 \mathrm{~kW}$. These savings were $11 \%$ of the whole building power and 0.30 $\mathrm{W} / \mathrm{ft}^{2}$. The maximum saving was achieved during the $2: 15 \mathrm{pm}$ to $2: 30 \mathrm{pm}$ interval, shortly after the $\$ 0.75 / \mathrm{kWh}$ price signal was received. Comparing the sites to each other, GSA achieved the largest shed $\mathrm{kW}(274 \mathrm{~kW})$, Roche achieved the largest shed \% (29\%), and Albertsons achieved the largest shed $\mathrm{kW} / \mathrm{ft} 2\left(0.75 \mathrm{~kW} / \mathrm{ft}^{2}\right)$ among the five sites. 
Table 4-14. Summary of Estimated Savings of All Site (Whole-Building Method)

\begin{tabular}{|c|c|c|c|c|c|c|c|c|c|c|c|c|c|c|c|c|c|}
\hline \multirow{3}{*}{\begin{tabular}{|l|} 
Time \\
Price signal \\
Albertsons
\end{tabular}} & $\begin{array}{r}\text { from } \\
\text { to }\end{array}$ & $\begin{array}{l}12: 45 \\
13: 00\end{array}$ & $\begin{array}{l}13: 00 \\
13: 15\end{array}$ & $\begin{array}{l}3: 15 \\
3: 30\end{array}$ & \begin{tabular}{|l|}
$13: 30$ \\
$13: 45$ \\
\end{tabular} & $\begin{array}{l}13: 45 \\
14: 00\end{array}$ & $\begin{array}{l}14: 00 \\
14: 15 \\
\end{array}$ & \begin{tabular}{|l|l|}
$14: 15$ \\
$14: 30$
\end{tabular} & $\begin{array}{l}14: 30 \\
14: 45\end{array}$ & $\begin{array}{l}14: 45 \\
15: 00\end{array}$ & $\begin{array}{l}15: 00 \\
15: 15\end{array}$ & $\begin{array}{l}15: 15 \\
15: 30\end{array}$ & \begin{tabular}{|l|l|}
$15: 30$ \\
$15: 45$ \\
\end{tabular} & & $\begin{array}{l}16: 00 \\
16: 15\end{array}$ & \begin{tabular}{|l|}
$16: 15$ \\
$16: 30$
\end{tabular} & $\begin{array}{l}16: 30 \\
16: 45\end{array}$ \\
\hline & $\$ / \mathrm{kW}$ & \multicolumn{2}{|c|}{0.10} & \multicolumn{4}{|c|}{0.30} & \multicolumn{4}{|c|}{0.75} & \multicolumn{4}{|c|}{0.30} & \multicolumn{2}{|c|}{0.10} \\
\hline & WBP kW & 351 & 320 & 318 & 330 & 351 & 332 & 326 & 320 & 323 & 344 & 332 & 341 & 345 & 345 & 347 & 347 \\
\hline & Shed kW & -1 & 24 & 25 & 25 & 12 & 22 & 18 & 27 & 37 & 10 & 10 & 8 & 13 & & 0 & \\
\hline & eed $\%$ & $0 \%$ & $7 \%$ & $7 \%$ & $7 \%$ & $3 \%$ & $6 \%$ & $5 \%$ & $8 \%$ & $10 \%$ & $3 \%$ & $3 \%$ & $2 \%$ & $4 \%$ & $2 \%$ & $0 \%$ & \\
\hline & Shed & -0.02 & 0.48 & 0.51 & 0.51 & 0.23 & 0.44 & 0.36 & 0.54 & 0.75 & 0.21 & 0.20 & 0.16 & 0.26 & 0.11 & 0.00 & 0.01 \\
\hline \multirow[t]{4}{*}{$B$ of $A$} & WBP kW & 720 & 726 & 729 & 717 & 752 & 751 & 723 & 746 & 742 & 731 & 726 & 718 & 721 & 706 & 682 & 664 \\
\hline & She & -7 & -15 & -13 & -2 & -31 & -28 & -8 & -33 & -31 & -17 & -15 & -6 & -18 & 13 & & 10 \\
\hline & WBP shed \% & $-1 \%$ & $-2 \%$ & $-2 \%$ & $0 \%$ & $-4 \%$ & $-4 \%$ & $-1 \%$ & $-5 \%$ & $-4 \%$ & $-2 \%$ & $-2 \%$ & $-1 \%$ & $-3 \%$ & $-2 \%$ & $1 \%$ & $1 \%$ \\
\hline & Shed W/spft & -0.03 & -0.07 & -0.06 & -0.01 & -0.15 & -0.13 & -0.04 & -0.16 & -0.14 & -0.08 & -0.07 & -0.03 & -0.08 & -0.06 & 0.03 & 0.05 \\
\hline \multirow[t]{4}{*}{ GSA } & WBP kW & 2248 & 2168 & 2093 & 2085 & 2091 & 2098 & 2086 & 2109 & 2178 & 2232 & 2184 & 2126 & 2085 & 2123 & 2178 & 2077 \\
\hline & Shed kW & 141 & 195 & 264 & 271 & 274 & 257 & 259 & 219 & 195 & 141 & 217 & 246 & 223 & 176 & 48 & 83 \\
\hline & WBP shed \% & $6 \%$ & $8 \%$ & $11 \%$ & $12 \%$ & $12 \%$ & $11 \%$ & $11 \%$ & $9 \%$ & $8 \%$ & $6 \%$ & $9 \%$ & $10 \%$ & $10 \%$ & $8 \%$ & $2 \%$ & $4 \%$ \\
\hline & Shed W/spft & 0.14 & 0.20 & 0.27 & 0.28 & 0.28 & 0.26 & 0.26 & 0.22 & 0.20 & 0.14 & 0.22 & 0.25 & 0.23 & 0.18 & 0.05 & 0.08 \\
\hline \multirow[t]{4}{*}{ Roche } & WBP kW & 658 & 587 & 569 & 558 & 555 & & 458 & 425 & 412 & 465 & 463 & 457 & 450 & 522 & 527 & 454 \\
\hline & Shed kW & 12 & 72 & 89 & 75 & 77 & 118 & 150 & 154 & 165 & 113 & 105 & 102 & 92 & 12 & 4 & 27 \\
\hline & WBP shed \% & $2 \%$ & $11 \%$ & $14 \%$ & $12 \%$ & $12 \%$ & $19 \%$ & $25 \%$ & $27 \%$ & $29 \%$ & $20 \%$ & $19 \%$ & $18 \%$ & $17 \%$ & $2 \%$ & $1 \%$ & $6 \%$ \\
\hline & Shed W/spft & 0.06 & 0.38 & 0.47 & 0.39 & 0.40 & 0.61 & 0.78 & 0.80 & 0.86 & 0.59 & 0.55 & 0.53 & 0.48 & 0.06 & 0.02 & 0.14 \\
\hline \multirow[t]{4}{*}{$\overline{U C S B}$} & WBP kW & 804 & 756 & 753 & 763 & 758 & & & 677 & 688 & 841 & 795 & 777 & 770 & 798 & 796 & 793 \\
\hline & Shed kW & -9 & 47 & 55 & 35 & 40 & 109 & 100 & 109 & 98 & -70 & -17 & -3 & -4 & -32 & -13 & -27 \\
\hline & WBP shed \% & $-1 \%$ & $6 \%$ & $7 \%$ & $4 \%$ & $5 \%$ & $14 \%$ & $13 \%$ & $14 \%$ & $12 \%$ & $-9 \%$ & $-2 \%$ & $0 \%$ & $-1 \%$ & $-4 \%$ & $-2 \%$ & $-4 \%$ \\
\hline & Shed W/spft & -0.03 & 0.16 & 0.19 & .12 & 0.14 & 0.38 & 0.35 & 0.38 & 0.34 & -0.24 & -0.06 & -0.01 & 0.02 & -0.11 & -0.05 & -0.09 \\
\hline \multirow[t]{4}{*}{ Total } & $\mathrm{P} \mathrm{kW}$ & & 4557 & 4462 & 4452 & 4507 & 4351 & 427 & 4276 & 4342 & 4612 & 4499 & 4418 & 4371 & 4494 & 528 & 4334 \\
\hline & ed kW & 136 & 323 & 421 & 405 & 371 & 477 & 519 & 476 & 465 & 178 & 301 & 346 & 305 & 149 & 44 & 94 \\
\hline & WBP shed \% & $3 \%$ & $7 \%$ & $9 \%$ & $8 \%$ & $8 \%$ & $10 \%$ & $11 \%$ & $10 \%$ & $10 \%$ & $4 \%$ & $6 \%$ & $7 \%$ & $7 \%$ & $3 \%$ & $1 \%$ & $2 \%$ \\
\hline & Shed W/spft & 0.08 & 0.19 & 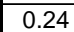 & 0.24 & 0.22 & 0.28 & 0.30 & 0.28 & 0.27 & 0.10 & 0.17 & 0.20 & 0.18 & 0.09 & 0.03 & 0.05 \\
\hline
\end{tabular}

WBP: $\quad$ whole building power

Shed: $\quad$ demand saving (= baseline demand - actual demand)

WBP shed \%: demand saving ratio in whole building power

Shed $\mathrm{W} / \mathrm{ft}^{2}$ : $\quad$ demand saving per square feet

Table 4-15. Summary of Estimated Savings of All Site (Component Method)

\begin{tabular}{|c|c|c|c|c|c|c|c|c|c|c|c|c|c|c|c|c|c|}
\hline \multirow{3}{*}{\begin{tabular}{|l|} 
Time \\
Price signal \\
Albertsons
\end{tabular}} & $\begin{array}{r}\text { from } \\
\text { to }\end{array}$ & $\begin{array}{l}12: 45 \\
13: 00 \\
\end{array}$ & \begin{tabular}{|l|l}
$13: 00$ \\
$13: 15$ \\
\end{tabular} & \begin{tabular}{|l|l|}
$13: 15$ \\
$13: 30$ \\
\end{tabular} & \begin{tabular}{|l|}
$13: 30$ \\
$13: 45$ \\
\end{tabular} & \begin{tabular}{|l|}
$13: 45$ \\
$14: 00$ \\
\end{tabular} & \begin{tabular}{|l|}
$14: 00$ \\
$14: 15$ \\
\end{tabular} & \begin{tabular}{|l}
$14: 15$ \\
$14: 30$
\end{tabular} & \begin{tabular}{|l|}
$14: 30$ \\
$14: 45$ \\
\end{tabular} & $\begin{array}{l}14: 45 \\
15: 00 \\
\end{array}$ & \begin{tabular}{|l|}
$15: 00$ \\
$15: 15$ \\
\end{tabular} & \begin{tabular}{|l|}
$15: 15$ \\
$15: 30$
\end{tabular} & \begin{tabular}{|l|}
$15: 30$ \\
$15: 45$ \\
\end{tabular} & \begin{tabular}{|l|}
$15: 45$ \\
$16: 00$ \\
\end{tabular} & \begin{tabular}{l|}
$16: 00$ \\
$16: 15$ \\
\end{tabular} & \begin{tabular}{|l|}
$16: 15$ \\
$16: 30$
\end{tabular} & \begin{tabular}{|l|}
$16: 30$ \\
$16: 45$ \\
\end{tabular} \\
\hline & $\$ / \mathrm{kW}$ & \multicolumn{2}{|c|}{0.10} & \multicolumn{4}{|c|}{0.30} & \multicolumn{4}{|c|}{0.75} & \multicolumn{4}{|c|}{0.30} & \multicolumn{2}{|c|}{0.10} \\
\hline & Shed kW & 0 & 22 & 26 & 26 & 26 & 39 & 41 & 41 & 41 & 30 & 26 & 26 & 26 & & -1 & 1 \\
\hline & WBP shed \% & $0 \%$ & $6 \%$ & $8 \%$ & $7 \%$ & $7 \%$ & $10 \%$ & $11 \%$ & $11 \%$ & $11 \%$ & $8 \%$ & $7 \%$ & $7 \%$ & $7 \%$ & $3 \%$ & $0 \%$ & $0 \%$ \\
\hline & Shed W/spft & 0.00 & 0.44 & 0.52 & 0.52 & 0.52 & 0.78 & 0.82 & 0.83 & 0.83 & 0.60 & 0.52 & 0.52 & 0.52 & 0.18 & -0.02 & -0.01 \\
\hline \multirow[t]{3}{*}{$B$ of $A$} & Shed kW & 0 & 0 & 6 & 11 & 7 & 1 & 8 & 8 & 0 & 0 & 9 & 12 & 0 & 0 & 0 & 0 \\
\hline & WBPs & $0 \%$ & $0 \%$ & $1 \%$ & $1 \%$ & $1 \%$ & $0 \%$ & $1 \%$ & $1 \%$ & $0 \%$ & $0 \%$ & $1 \%$ & $2 \%$ & $0 \%$ & $0 \%$ & $0 \%$ & $0 \%$ \\
\hline & Shed W/spft & 0.00 & 0.00 & 0.03 & 0.05 & 0.03 & 0.00 & 0.04 & 0.04 & 0.00 & 0.00 & 0.04 & 0.06 & 0.00 & 0.00 & 0.00 & 0.00 \\
\hline \multirow[t]{3}{*}{ GSA } & Shed kW & 75 & 164 & 185 & 161 & 204 & 165 & 192 & 156 & 146 & 149 & 238 & 240 & 223 & 109 & 40 & 54 \\
\hline & WBP shed \% & $3 \%$ & $7 \%$ & $8 \%$ & $7 \%$ & $9 \%$ & $7 \%$ & $8 \%$ & $7 \%$ & $6 \%$ & $6 \%$ & $10 \%$ & $10 \%$ & $10 \%$ & $5 \%$ & $2 \%$ & $3 \%$ \\
\hline & Shed W/spft & 0.08 & 0.17 & 0.19 & 0.16 & 0.21 & 0.17 & 0.20 & 0.16 & 0.15 & 0.15 & 0.24 & 0.25 & 0.23 & 0.11 & 0.04 & 0.05 \\
\hline \multirow[t]{3}{*}{ Roche } & Shed kW & 0 & 70 & 70 & 70 & 70 & 107 & 107 & 107 & 107 & 70 & 70 & 70 & 70 & & 0 & 0 \\
\hline & WBP shed \% & $0 \%$ & $11 \%$ & $11 \%$ & $11 \%$ & $11 \%$ & $18 \%$ & $19 \%$ & $20 \%$ & $21 \%$ & $13 \%$ & $13 \%$ & $13 \%$ & $14 \%$ & $0 \%$ & $0 \%$ & $0 \%$ \\
\hline & Shed W/spft & 0.00 & 0.37 & 0.37 & 0.37 & 0.37 & 0.56 & 0.56 & 0.56 & 0.56 & 0.37 & 0.37 & 0.37 & 0.37 & 0.00 & 0.00 & 0.00 \\
\hline \multirow[t]{3}{*}{ UCSB } & Shed kW & -2 & 37 & 51 & 40 & 55 & 117 & 129 & 123 & 119 & -19 & 1 & 15 & 17 & -19 & -3 & -17 \\
\hline & WBP shed \% & $0 \%$ & $5 \%$ & $6 \%$ & $5 \%$ & $7 \%$ & $15 \%$ & $16 \%$ & $15 \%$ & $15 \%$ & $-2 \%$ & $0 \%$ & $2 \%$ & $2 \%$ & $-2 \%$ & $0 \%$ & $-2 \%$ \\
\hline & Shed W/spft & -0.01 & 0.13 & 0.18 & 0.14 & 0.19 & 0.40 & 0.45 & 0.43 & 0.41 & -0.07 & 0.01 & 0.05 & 0.06 & -0.07 & -0.01 & -0.06 \\
\hline \multirow[t]{3}{*}{ Total } & Shed kW & 73 & 294 & 338 & 307 & 362 & 429 & 477 & 436 & 414 & 231 & 344 & 363 & 337 & 98 & 36 & 36 \\
\hline & WBP shed \% & $2 \%$ & $6 \%$ & $7 \%$ & $6 \%$ & $7 \%$ & $9 \%$ & $10 \%$ & $9 \%$ & $9 \%$ & $5 \%$ & $7 \%$ & $8 \%$ & $7 \%$ & $2 \%$ & $1 \%$ & $1 \%$ \\
\hline & Shed W/spft & 0.04 & 0.17 & 0.20 & 0.18 & 0.21 & 0.25 & 0.28 & 0.25 & 0.24 & 0.13 & 0.20 & 0.21 & 0.20 & 0.06 & 0.02 & 0.02 \\
\hline
\end{tabular}

\section{Impact of Early Implementation and End of Shed Strategies}

As a general trend, all sites except B of A responded approximately 15 minutes earlier than when the price signal took effect (B of A's demand shed was too small to identify the result). For example, Albertsons reduced the demand a few minutes after 1:00 pm while the price signal rose at 1:15 pm. The system also responded 15 minutes earlier when the price signal fell. The price signal is designed to inform 15-minute ahead price 
(for example, the signal received at 1:00 pm tells the future price of 1:15 pm). This was in order to give the systems preparation time to change their operation before the price change. The result indicates these sites could respond to the signal and changed the operation within a few minutes. The issue is that the shed strategies were disabled as soon as the system received the lower price signal, and therefore the demand increased 15 minutes before the high price period ended. This may cause higher electricity charges despite of the demand response effort.

\section{Demand Shed Related to Price Signal}

Figure 4-19 and Figure 4-20 show shed $\mathrm{kW}$ and shed W/ft ${ }^{2}$ of each site averaged by each 1-hour price signal level. The largest shed was at GSA, maintaining over $150 \mathrm{~kW}$ during the entire shed period. The greatest demand shed intensity was at Albertsons, with 0.4 to $0.7 \mathrm{~W} / \mathrm{ft}^{2}$ during the shed period. All but the B of A site shed at least $0.15 \mathrm{~W} / \mathrm{ft}^{2}$.

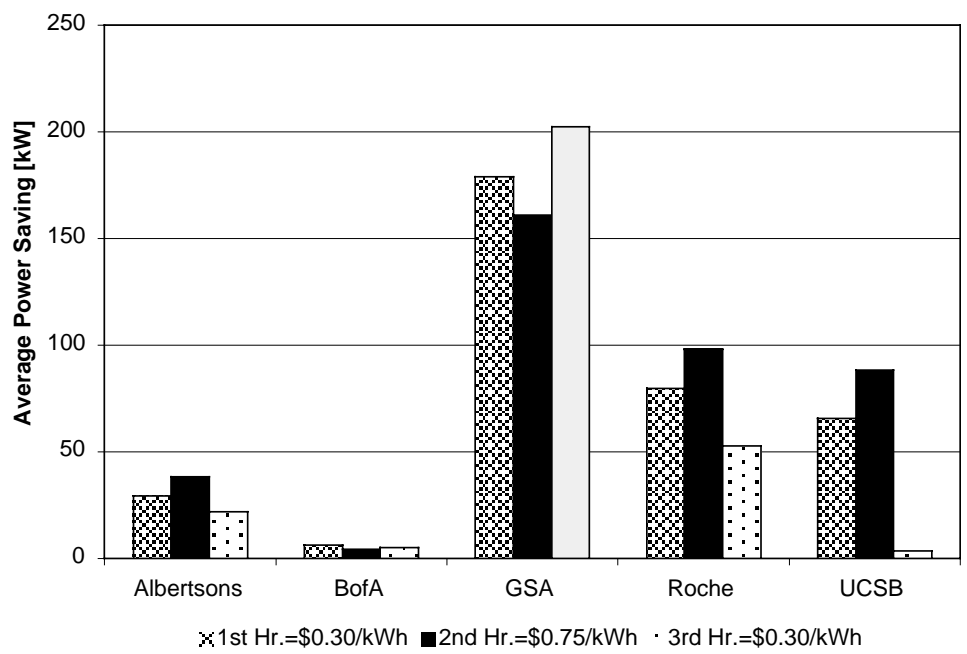

Figure 4-37. Average Shed kW by Price Signal Level (Component Method)

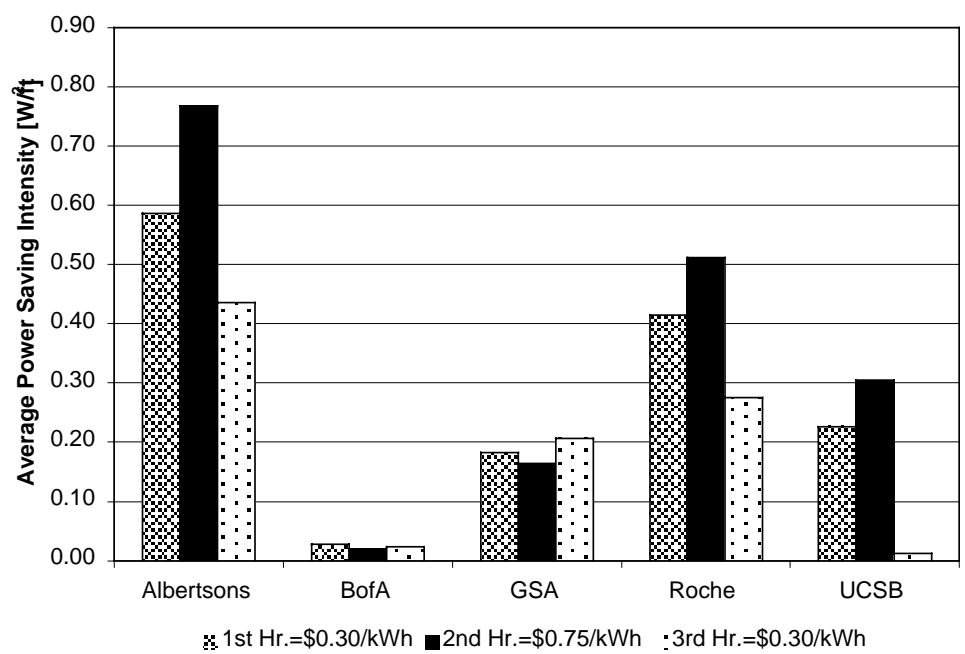

Figure 4-38. Average Shed W/ft ${ }^{2}$ by Price Signal Level (Component Method) 


\section{Whole Building Power and Aggregated Savings}

Figure 4-21 shows the actual whole building power of all five sites and aggregated demand savings, calculated by the component method. The total demand reduction began at 1:00 pm (15 minutes before $\$ 0.30 / \mathrm{kWh}$ period) and were further reduced at 2:00 pm (15 minutes before $\$ 0.75 / \mathrm{kWh})$. The systems returned to the same shed strategies used at the previous $\$ 0.30 / \mathrm{kWh}$ level at 3:00 pm. The systems returned to normal operation at 4:00 pm.

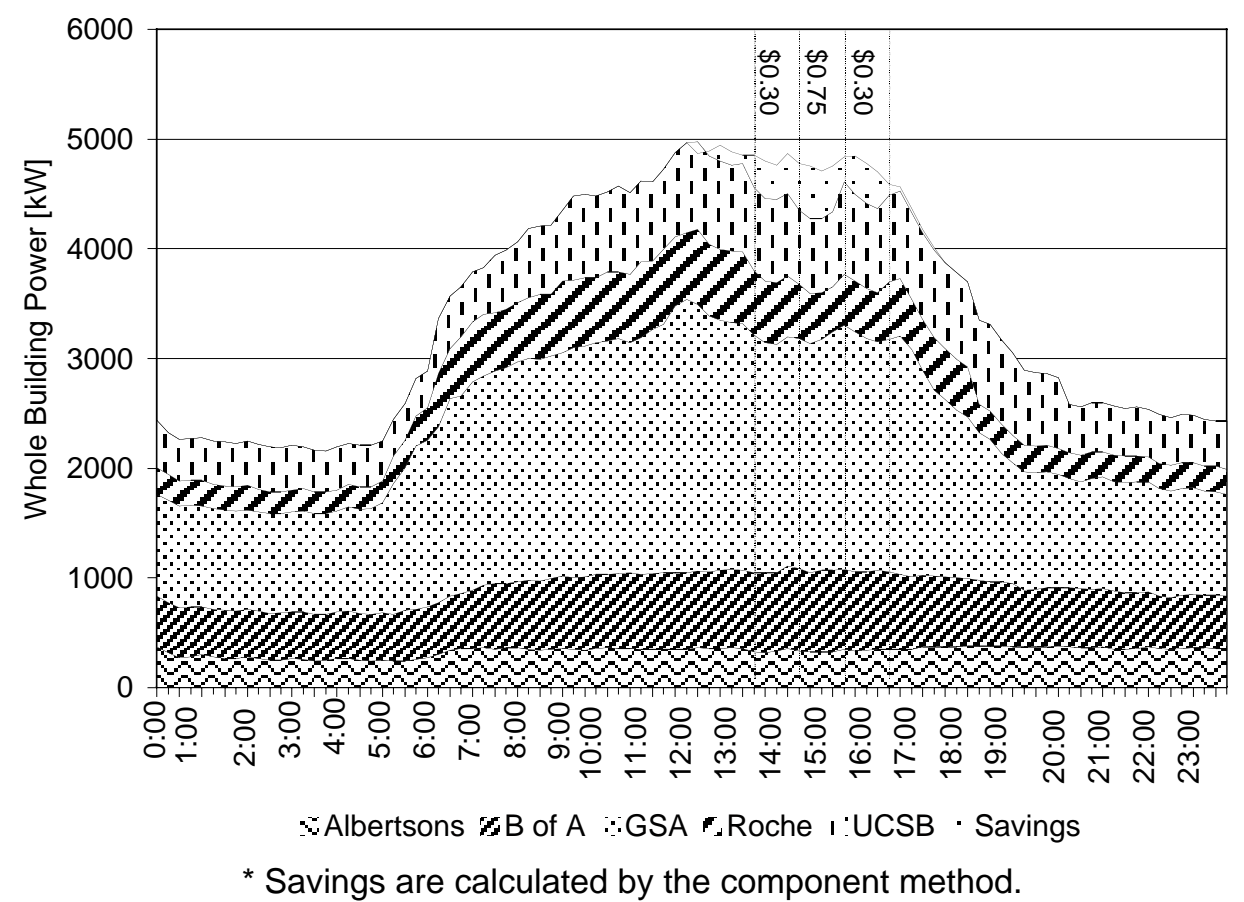

Figure 4-39. Summary of All Sites

Demand saving by the whole-building method and the component method were similar, though the similarities vary by site. Figure 4-22 shows a comparison of the wholebuilding method and the component method calculated shed $\mathrm{kW}$ for each site. Roche's whole-building method savings is higher than that calculated by the component method, because the whole-building baseline was affected by an operational change of one building in the site. The overall results suggest that the weather normalized wholebuilding profiles provide reasonable estimates. Whole building data are much easier to collect and are more readily available than end-use and component data. Previous work has shown that methods that do not account for weather have significant bias for large commercial buildings (KEMA-Xenergy, 2003). We did not compare the weathernormalized whole-building analysis method with the flat 10-day average baseline used in many DR evaluations. 


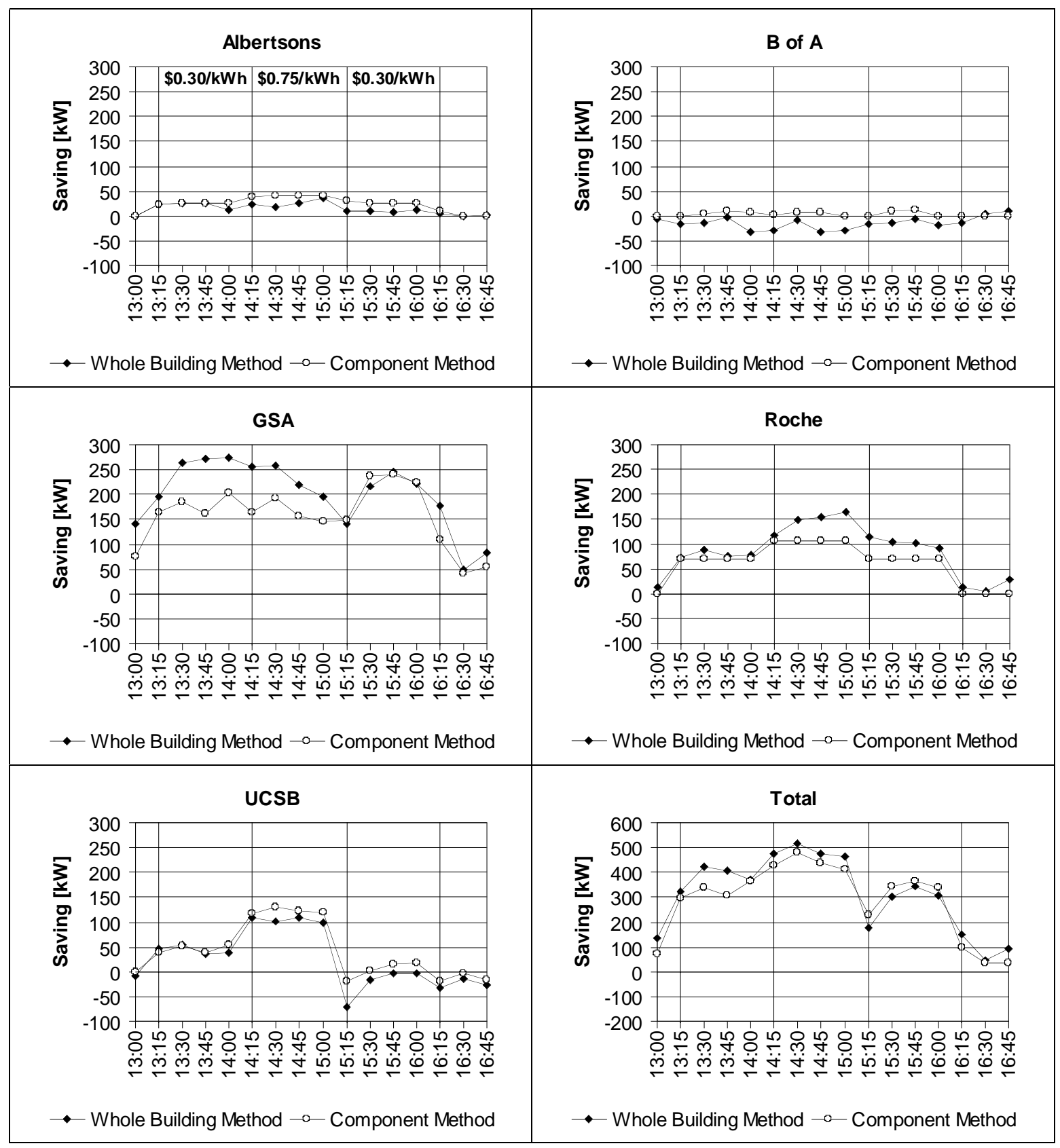

Figure 4-40. Saving Estimates by Whole-Building Method and Component Method 


\section{Discussion: Organizational and Technological Issues}

This section provides a discussion of the key lessons learned from this Auto-DR test. They have been organized into four main topic areas:

- Implementation and organizational challenges in deploying Auto-DR technology

- Technical challenges at each site

- Key lessons learned concerning unique attributes of the systems at each site that further our understanding of the diversity of technology infrastructures among these facilities

- State-of-the-art technology and desirable features for future Auto-DR systems

\section{5-1 Implementation and Organizational Challenges}

There are a variety of implementation and organizational challenges that had to be addressed during the execution of this project. After the research team defined the highlevel objectives of the project, these objectives were conveyed to the key decision makers at each site as part of the recruitment effort. Facility and energy managers who were in the position to understand and approve of a project of this magnitude tended to be spread thin between important management responsibilities. As these managers delegated project responsibilities to their staffs, progress often stalled. The tasks necessary to implement Auto-DR systems at each site cut across several areas of expertise and authority. To implement Auto-DR systems as designed in the November 2003 test, most sites required input from facility managers, HVAC experts, EMCS programmers, Web services programmers and IT managers. Defining, designing, coordinating and implementing the details of each site's system often required substantial effort from the facility staff and the research team.

Web services is a relatively new technology, and most of the development teams had not used it prior to the test. But because of the simplicity of this technology, lack of experience was not a major impediment to success for any of the Auto-DR test development teams. However, even though learning Web services is simple for most programmers in the IT community, development of this technology requires programming skills beyond the ability of most EMCS "programmers". Within most EMCS field organizations, it is uncommon for EMCS programmers to broaden their skills to include IT level programming expertise.

Within each Auto-DR test team, an IT professional programmed the Web services client and business logic, while an EMCS professional programmed or configured the shed logic. While this approach ultimately produced successful results, the added organizational complexity made scoping, development, testing and project management more difficult than if all of the required software changes could be conducted by one person or organization. 


\section{5-2 Overview of Technical Challenges}

There were a number of specific technical challenges in conducting this test. This section describes four key issues: software compatibility, time zones, IT and EMCS failures, and hardware issues.

\section{Software Compatibility}

Platform independence is one of the key attributes of well-designed Web services. However, platform dependencies are not always easy to spot. In the Auto-DR test, there were compatibility issues associated with the server software that was developed within the Microsoft ".Net" environment and two of the clients that were developed in Java or Delphi environments. These problems were not caused by inherent platform incompatibilities per se, but by the indiscriminate use of a text format rather than another format more commonly used to display data. The problems were resolved by modifying the Web services server to use a format that is more commonly used for data.

\section{Time zones}

The developers, users, price server and polling clients were distributed across 3 time zones in continental North America. Thus, a common universal time zone was required for use by all parties. Coordinated Universal Time $(\boldsymbol{U T C})$ was selected as the standard. All parties used UTC in the lowest software levels, but most users preferred to see their local time zone when interacting with the system on a HMI. In the test, programmers often converted UTC to local time to improve the usability of time related data (e.g., 20:01 UTC was converted to 12:01 pm PST). But since this work was done without regard for users in other time zones, some time indication problems occurred with the viewing and logging at various sites. These problems were resolved by adopting a twopronged approach; UTC was used for low level software and PST was used where time data would be viewed or logged.

\section{IT Failures}

In several cases, portions of the IT infrastructure failed for various reasons. At Albertsons, the polling client software stopped polling the price server inexplicitly just prior to the start of the test window. It was restored minutes later after system administrators were alerted by telephone. An upgrade of the UCSB EIS software several weeks before the test left the gateway software out of synch. Since the remote control functionality was not functional in time for the test, a remotely controlled IP Relay was added to provide the control interface to the EMCS. During the first test window, B of A's monitored building was not commanded into shed mode for unknown reasons.

\section{EMCS Failures}

In the first test window, two sites failed to properly enter shed mode due to failures in the EMCSs. At GSA, an I/O controller locked up and failed to initiate a shed mode. At UCSB, the first test was marred by some EMCS points being "fixed" in manual mode, unable to automatically respond to remote commands to enter certain shed strategies. 


\section{Hardware Issues}

During the installation and early testing phase of the project, Albertsons management reported that the chicken cooker lost power during the shed test of the freezer case lights. Further research of the electric trend logs showed an intermittent load profile that was consistent with that of an electric cooker on the same circuit as the freezer case lights. The cooker profile appeared several times per day starting several months prior. Albertsons management verified that the chicken cooker was installed earlier that summer on the date that the mysterious load profile was first observed. Rather than perform costly revisions to high voltage wiring, the problem was resolved by modifying the EMCS software so as to remove the chicken cooker and freezer case lights from the shed strategy.

\section{5-3 Lessons from each Site}

This section identifies lessons learned based on the unique attributes of each participating facility. Issues related to the controls and communications infrastructures and shed strategies are discussed for each system.

\section{Albertsons System Overview}

The Albertsons building telemetry data system is split between two systems. The EMCS (excluding electric monitoring) is accessed via dial-up modem. EIS data are available via any Web browser through the Engage/eLution site. The lack of integrated systems makes energy management and analysis more difficult for facilities staff.

Albertsons' enterprise networks and control networks are tightly integrated. Albertsons' corporate WAN is used communicate between the business logic/polling client and the on-site IP I/O device. This enterprise network is also used for mission critical point of sale data communications within the Albertsons organization. The fact that the energy data are shared and communicated over the mission critical enterprise network indicates a high level of trust and collaboration between Albertsons core business managers and Albertsons energy managers.

\section{Albertsons Shed Strategy Impacts}

The shed strategy was not objectionable to the store managers or patrons. Although the transition between $100 \%$ overhead lighting to $50 \%$ was noticeable, there were no complaints. The reduction of overhead light appeared to make the other light sources in the store, such as case lights, seem more intense. There is no evidence that the freezer doors fogged up during the shed, even though the anti-sweat heaters were switched to night mode. If the transition of overhead lights to $50 \%$ were gradual (e.g., through use of dimmable ballasts) the entire shed would probably not be noticeable. 


\section{Bank of America System Overview}

The enterprise networks and control networks are tightly integrated. B of A's corporate WAN is used to communicate across the country to the on-site gateway. This network is also used for mission critical financial data communications within the B of $\mathrm{A}$ organization. Like Albertsons, the fact that the energy data are shared and communicated over the mission critical enterprise network indicates a high level of trust and collaboration between B of A's core business managers and B of A's energy managers. The use of highly secure and reliable hardware VPN routers and the use of a co-location (co-lo) to host the polling client and business logic computers are indications that system availability and security are high priorities to $\mathrm{B}$ of $\mathrm{A}$.

When the WebGen system takes action to reduce energy at its connected sites, it uses a centralized control paradigm. While demand response systems are inherently centralized (signals to shed loads are generated in a one-to-many relationship), centralized control for day-to-day operation is less common.

In most control system markets (commercial buildings, industrial controls, etc.) there has been a trend for several decades toward distributed or de-centralized control. In distributed control architectures, the control logic is physically moved as close to the sensors and final control elements (relays, valves, etc.) as possible. Distributed control systems have traditionally been less costly, more flexible and more robust than centralized systems. However, in the IT community there has been a movement toward hosted solutions, application service providers (ASPs) and other centralized solutions. Ubiquitous Internet connectivity and advances in IT equipment have made these systems less costly, more flexible and more robust for certain applications.

The WebGen system alternates between centralized and decentralized paradigms on cycles of about twenty minutes. One minute a fan system maintains a setpoint entered by onsite building operators. In the next minute, a neural network algorithm defines the setpoint from over 3000 miles away.

\section{B of A Shed Strategy Impacts}

With regard to the shed strategy employed at this site, there is no evidence that the modest reduction in duct static pressure for durations of twenty minutes caused any negative comfort effects to the occupants during the test. However, as shown in the measured data, the extent of the electric demand shed was also negligible.

If this strategy were expanded to produce significant electric sheds, this method may pose some fundamental drawbacks. In VAV systems, when duct static pressure is reduced below the minimum required by the terminal boxes, airflow is reduced in the zones. But the reduction is not shared evenly between all the boxes. The zones of greatest demand are the ones that are starved for air most immediately and most severely. In the B of A building tested, the potential problem is exacerbated by the lack of sensors. Fan airflow is not measured and only nine "representative" zone temperature sensors are available for 
the entire $211,000 \mathrm{ft}^{2}$ building. There are not enough sensors to estimate the effects that reductions in airflow would have on occupants.

\section{GSA System Overview}

The enterprise and EMCS infrastructures used to enable Auto-DR at this site are linked together in a long series of serial components and communication links. The prototype system was assembled using spare parts at a low cost. With so many links, it is not surprising that there was a communication failure due to an unexplained equipment lockup during the first test. To make the system more robust, a review of the components and architecture should be conducted.

\section{GSA Shed Strategy Impacts}

The second test was quite successful as communications were functional from end-toend. The shed strategy produced an electric shed about as large as the other four sites combined. Because the temperature setpoint reset was at the zone level, comfort for each occupant could be maintained within the revised, relaxed constraints. To implement this strategy, it was necessary to revise the software parameters and some logic in each of the 1,050 VAV terminal box controllers. For most EMCS systems, the labor required to make these revisions would be substantial (one to three weeks). In this building, the process had been somewhat automated by previous system upgrades. This allowed EMCS reprogramming for Auto-DR to be conducted in about three hours.

\section{Roche System Overview}

The Tridium Niagra framework ties together the various EMCS protocols in a seamless fashion. The Web interface provides operators compete monitoring and control capability from anywhere on the campus. It was relatively straightforward to interface the Auto-DR polling client and associated business logic to the system. The most challenging part of the project was setting up an "extra" computer outside of the Roche firewall in the DMZ. This design allowed secure communications between the price server on the public Internet, the polling client on the DMZ computer and the Tridium computer inside the corporate network.

\section{UCSB System Overview}

Remote monitoring and control of the EMCS and EIS data were available over the Internet prior to the Auto-DR test. However, at the time of the test, remote control of the EMCS was not available. The software gateway between the enterprise network and the EMCS network lost remote-control functionality during an "upgrade" of the Silicon Energy server software. To meet the schedule of the Auto-DR test, in lieu of a gateway, an IP I/O controller was added to allow the Auto-DR business logic to initiate remotecontrol functions including shed initiation. 


\section{UCSB Shed Strategy Impacts}

The shed strategy proved to be very effective. The books and other mass in the Library buildings act as a thermal "flywheel" to help keep the space comfortable during the shed periods. In addition, the sheds reduced airflow without shutting off fans completely. The Santa Barbara's coastal climate helped provide a temperate airflow even though the cooling valves were closed to save chiller energy.

\section{5-4 State of the Art Auto-DR Systems}

This section describes the attributes of state-of-art Auto-DR systems based on technologies that were either demonstrated or otherwise available at the time of the November 2003 Auto-DR test. A truly state-of-the-art Auto-DR system would use the "best of the best" components, systems and strategies from end to end. Such a system would be designed from scratch to meet a very specific set of requirements driven by business cases or other factors. The "best" system would meet or exceed the requirements at the lowest installed cost.

\section{Flexible Designs for the Future}

Today's state-of-the-art Auto-DR technology could be applied in many different ways, depending on the scenarios and use cases that they are designed to satisfy. As the scenarios, use cases, and driving forces behind Auto-DR become better defined, systems will be designed and deployed accordingly. Since these design criteria are likely to remain in flux, Auto-DR system flexibility and future-proofing are a very high priorities.

\section{Features}

Customers should have numerous options about how they can participate in Auto-DR programs. For any given motivating force that drives customers to consider Auto-DR (e.g., price), each will have different circumstances under which they will want to participate. Customer flexibility must be built into any state-of-the-art Auto-DR system. They should have the ability to use custom business logic that is applicable to their own operations. Some may choose to allow remote real-time sheds, while others may want some advanced warning via pager or cell phone and the ability to opt out, if desired. Other important features in state-of-the-art Auto-DR systems are two-way communications, real-time monitoring, high security, and high system availability.

\section{Leveraging Trends in Technology}

The lower the installed cost of state-of-the-art Auto-DR systems, the sooner they will find their way into mainstream use. One of the most important ways to keep costs low is to leverage existing trends in technology. The use of existing IT technology in Auto-DR systems wherever possible is an important way to keep costs low. The public Internet and private corporate LAN/WANs are ideal platforms for Auto-DR controls and communications due to their ubiquity, especially in large commercial buildings. In addition, the performance of IT equipment (e.g., routers, firewalls, etc.) continues to improve and equipment prices continue to drop. Auto-DR systems based on standard IT platforms will also tend to be more scalable and secure than special purpose systems developed specifically for Auto-DR. 


\section{Enterprise, EMCS and EIS Integration}

Another way to obtain high system performance and keep the system costs low is through increased integration within the building. Since energy data from EISs are simply another type of measured data, EISs and EMCSs should share the same networks so as to maximize system performance and functionality and minimize cost. In addition to eliminating a redundant EIS network, other aspects of the system are also unified through this approach. Use of an integrated EMCS/EIS database and associated archiving and visualization tools increases user functionality while reducing cost. The ability to change setpoints for HVAC equipment and observe and analyze the effect on electricity use from the same HMI is an important enhancement to both the EMCS and EIS.

State-of-the-art Auto-DR systems should have tight integration between the EMCS/EIS network and the enterprise networks within buildings. This strategy maximizes the performance, distribution and availability of the building data, while minimizing the installation and maintenance costs. In systems in which EMCSs and EISs are highly integrated with enterprise networks and the Internet, managing the flow of information is greatly simplified.

Once remote telemetry to EMCS and EIS is connected via enterprise networks, additional functionalities are enabled. While the need for localized field maintenance remains, geographic limitations on the distribution of building information virtually disappear. Reliable, distributed information about the operation of EMCS/EISs can actually reduce maintenance costs. By remotely determining operating conditions, equipment run-time, and error codes, maintenance workers can be dispatched in a more efficient fashion. In addition, once the integrated enterprise EMCS/EIS infrastructure is in place, fault detection and diagnostic (FDD) programs and other software tools can further reduce ongoing operational costs. Other remote telemetry applications include energy management, energy forecasting, aggregation, access control and regulatory record keeping.

In a state-of-the-art Auto-DR system, remote telemetry to the EMCS/EIS should be provided via password protected Web servers. Various levels of access should be provided for different types of users. Maintenance workers and accountants may only need monitoring access, while facility engineers may require complete control over setpoints and programming logic.

The network architecture of today's state-of-the-art Auto-DR system would tend to be flatter than that for most of the sites in the November 2003 test. A flat architecture is one in which there are a minimum number of layers of control network protocols between the front-end HMI and final control and monitoring elements such as actuators and sensors. The most robust and least costly systems should have no more than one enterprise network protocol and one control network protocol.

\section{Open Standards}

For flexibility and future-proofing as well as the option to choose "best of breed" products, state-of-the-art Auto-DR systems should use open standards wherever possible. Unlike proprietary systems, truly open systems are interoperable. In other words, a 
device from one company (e.g., Cisco) will easily and naturally reside on a network with products from other companies (e.g., Nortel). Most products in enterprise networks are interoperable. They all communicate using the TCP/IP protocol and can be set up and managed using common network management tools.

TCP/IP is clearly the worldwide protocol of choice for LAN/WANs, the Internet and enterprise networks. There are several open standard control network protocols including BACnet (ASHRAE Standard 135-2001) and LonTalk (ANSI/EIA/CEA 709.1). Although the use of the meta-language XML is becoming a standard framework for communicating over enterprise networks and the Internet, XML alone does not define data formats that can be used to convey measured building or energy data. Organizations such as $\boldsymbol{o B I X}$, BACnet XML and OPC XML are developing standards of this type.

\section{Shed Strategies}

State-of-the-art shed strategies should be designed to minimize discomfort, inconvenience, and loss of revenue for the participating sites. Shed strategies should be devised by customers to meet their needs. In general, shed strategies that use fine granularity closed-loop control are less likely to negatively impact building occupants for a given demand shed. Ideally, sheds would vary commensurately with a variable shed signal. Transitions should be fast enough to be effective, but slow enough so that transitions are not noticed by the building occupants.

In addition to HVAC control strategies, lighting and other switch-able loads should be considered for sheds as well. By increasing the Auto-DR controlled load to the point where it approaches the whole building load, each load type (e.g., HVAC, lighting, etc.) would need to shed a smaller amount in order to achieve a given shed target for the whole building. 


\section{Summary and Future Research Topics}

\section{General Findings and Research Issues}

In general, this study has shown that Auto-DR is technically feasible, although considerable effort from the research team was needed to guide the development of this technology at several of the case study sites. This study has obtained detailed information concerning numerous topics regarding DR and Auto-DR. We have made considerable progress in characterizing DR controls and communications systems for large facilities and in evaluating the specific DR shed strategies. We've also made extensive progress in developing a preliminary conceptual design for a communications infrastructure using XML and Web services. The technology presented here offers a glimpse of what may be possible for a large-scale deployment effort. We have also made progress in developing methods to measure and quantify the DR shed. The whole building and component level analysis methods showed good agreement with each other, allowing for flexibility in the level of monitoring required to quantify the shed impacts.

It is important to outline key outstanding issues that should be addressed in future research. One key question is how these strategies and technologies would perform in warm weather that is more typical of DR events. There were no complaints recorded from these DR strategies. However, the buildings were not aggressively "pushed" to shed large amounts of power. Facility managers may require payment for larger interventions and tests. A related issue is how both the communications and control technology infrastructure and the DR strategies can be used in today's DR programs in California.

While the DR strategies used at each site were developed on a custom basis, there is a need to gain more knowledge with how these strategies can be generalized. This knowledge will require market assessment of the capabilities of controls and communications systems, including remote connectivity and linkages between zones and central systems. As mentioned, there may be other strategies to help maximize DR and minimize the loss of services. A broader analysis of DR strategies is needed.

Perhaps the largest outstanding issue not addressed in this study is the costs and benefits of bringing Auto-DR functionality to typical existing buildings and industrial facilities. Evaluating such costs will be highly dependent on a good characterization of communications and control systems in today's facilities. Further work on characterizing the costs for the existing systems is needed to help understand the total costs and benefits of such technology. The benefits include energy savings, and there is a need to keep energy efficiency on the forefront of this area's research efforts to ensure the technology has dual use and is used in a optimal manner. Assessing the benefits of DR or Auto-DR will require a careful analysis of DR programs and developing scenarios that explore how large facilities might participate in these programs. These scenarios should consider realtime pricing, critical peak pricing, demand bidding, and other DR tariffs and programs. The economic perspectives can consider both the end-use customer and the societal costs and benefits of DR and Auto-DR. 


\section{Need for Technical Resources}

As a technology-research oriented project, the research team developed new knowledge about DR that is not widely available. DR is a complex concept. Facility operators need to understand DR economics, controls, communications, energy measurement techniques, and the relation between changes in operation and electric demand. Such understanding may involve numerous people at large facilities. Facility managers need good knowledge of controls, and current levels of outsourcing of control services complicate understanding of control strategies and system capabilities.

Examples of information needed to facilitate DR in general include guides on DR strategies and measurement and analysis tools and techniques to predict and measure DR. These tools should include building operational information and economic analysis tools. DR functional and commissioning tests are needed to guide facility managers through the process of identifying and testing DR strategies. Such techniques should ideally be linked to retro-commissioning guides that link energy savings and control optimization to DR.

One key finding of our research was that the controls and communications systems are complex. Limited information at each site was available to characterize the linkages and technology infrastructure. Facility managers need improved facility documentation as the industry attempts to push today's often broken control systems into advanced applications like DR. If we achieve success in understanding how to specify and describe these controls and communications infrastructures, it may prove useful to require such improved documentation in building codes of the future. Similarly, future work should explore requiring circuiting of non-critical end-uses and control strategies in new buildings to facilitate DR in new buildings through the building code process.

\section{Organizational Findings and Future Directions}

Providing energy is a complex endeavor. Making sure that every customer has secure, reliable and safe power is a tremendous accomplishment of modern civilization. The provision of such power has relied on a paradigm that is changing. Today, good energy services imply not merely providing safe, uninterruptible power, but supporting demand responsiveness. The present energy industry does support these goals, although there is universal acknowledgement among the five Auto-DR participants that these programs are necessary. These Lead Users cannot provide the leadership to develop DR technology on their own, although they are excellent partners for continuing research.

There are few industries and institutions that do not have Lead Users. In the case of Demand Response, the Lead Users may not identify themselves. One reason for this is that Demand Response programs are not mature in California. Facility managers value safety and security of information over demand responsiveness. It will be important for the researchers to target clusters of lead users and educate them about the long term likelihood of demand response programs and guide mid-level managers through their cumbersome bureaucracies towards participation in these programs. 
Future related research will need to evaluate the costs and benefits of new demand response programs and tariffs in California. Such information should be provided to the existing Lead Users to ensure that they become part of the extended demand response research team. They should be encouraged to share some of the financial burden to participate in DR, but their costs should remain low until DR has been economically proven. They are unlikely to have continued interest in this work unless more lucrative Demand Response programs develop. The lack of any mature Demand Response programs is an impediment to the research. Another impediment is that all five Lead Users purchase energy from Direct Access suppliers.

Understanding and facilitating DR requires a long-term perspective, and technical assistance will be needed for large commercial and industrial facilities. One promising avenue to deliver technical services is to partner with knowledgeable vendors and controls suppliers. Facility managers and owners who fully understand their outsourced technologies are rare, but an educated vendor can provide information and facilitate change more easily. Further, an educated vendor can supply the essential information on costs that the midlevel managers require.

\section{Technology Issues}

There are several key issues about future Auto-DR technology.

Scalability - While the Auto-DR infrastructure and associated components performed exceptionally in this test, additional technical issues associated with a full-scale Auto-DR deployment are expected to be significant. Additional research is required to address issues associated with deploying a real system in thousands or tens of thousands of sites. Scalable designs must consider installation and configuration methods, network management, version control and other long-term maintenance issues that have not been considered in the scope of this test.

Security - What are the worst-case scenarios that could occur if an Auto-DR system were breeched by hackers? What security design and process measures should be considered in a deployable Auto-DR system?

Use Cases - What are the likely use cases for which a deployable Auto-DR system should be designed? A system designed primarily for emergency load relief may be substantially different then a system designed to minimize operational costs on an ongoing basis. What are the shed timing requirements of various use cases and what are the timing characteristics of various Auto-DR systems and strategies?

Candidate Sites - What are the characteristics of a facility that make it a good candidate for Auto-DR? What are the characteristics of facility management and engineering teams that will help assure that their sites would succeed in Auto-DR programs? What are the most practical ways to add Auto-DR to existing commercial buildings that lack existing remote telemetry to HVAC systems? 
Intelligent Centralized Orchestration - If thousands of Auto-DR sites across the state were "on-line" how should they be orchestrated? From a centralized operations center, what criteria would determine which sites or groups of sites should be commanded to shed? To what degree could centralized automation act as a "traffic cop" to help the capacity meet the demand in each region? Is it possible to create flexible groupings customized to meet the needs of any given shed event? What would be the characteristics of such a system?

Technology Trends - As building systems including EMCS, EIS, security, fire and telephones are all moving toward inclusion into an enlarged more integrated definition of the "enterprise network", the technologies used in the enterprise are shifting as well. How will these trends affect the future of Auto-DR? How will wireless devices be used in Auto-DR? How will the eventual implantation of the next Internet protocol, IPv6 affect Auto-DR? 


\section{References}

California Energy Commission. 2003. “2003 Integrated Energy Policy Report. Docket \#02-IEP-01". Adopted by the Commission November 12, 2003. California Energy Commission publication number 100-03-019F.

Flynn, P. ( Editor). 2003. “The XML FAQ.” Maintained on behalf of the World Wide Web Consortium's XML Special Interest Group, version 3.01 (publication number 200301-14).

Goldman, C.A., M. Kintner-Meyer, and G. Heffner. 2002. "Do "Enabling Technologies" Affect Customer Performance in Price-Responsive Load Programs?" Lawrence Berkeley National Laboratory, Berkeley, CA. LBNL-50328.

Goldman, C.A., N. Hopper, O. Sezgen, M. Moezzi, R. Bharvirkar, B. Neenan, R. Boisvert, P. Cappers, and D. Pratt. 2004. "Customer Response to Day-ahead Wholesale Market Electricity Prices: Case Study of RTP Program Experience in New York." Prepared for The California Energy Commission. LBNL Report (Forthcoming).

International Performance and Measurement Verification protocol (IPMVP). 2001. "IPMVP Volume I: Concepts and Options for Determining Energy and Water Savings."

KEMA-Xenergy. 2002. "Enhanced Automation: Technical Options Guidebook." California Energy Commission publication number 400-02-005F.

KEMA-Xenergy. 2003. "Protocol Development for Demand Response Calculation Findings and Recommendations: Consultant Report". California Energy Commission publication number 400-02-017F.

Kinney, S., M.A. Piette, L. Gu, and P. Haves. 2001. "Demand Relief and Weather Sensitivity in Large California Commercial Office Buildings." Proceedings of the 2001 International Conference for Enhanced Building Operations. LBNL-48285.

Kintner-Meyer, M., C. Goldman, O. Sezgen, and D. Pratt. 2003. "Dividends with Demand Response." ASHRAE Journal, October.

Koomey, J., and R. Brown. 2002. "The Role of Building Technologies in Reducing and Controlling Peak Electricity Demand.” LBNL-49947. September.

Motegi, N., M.A. Piette, S. Kinney, and K. Herter. 2003a. "Web-based Energy Information Systems for Energy Management and Demand Response in Commercial Buildings.” LBNL Report 52510.

Motegi, N., and M.A. Piette, S. Kinney, and Jim Dewey. 2003b. "Case Studies of Energy Information Systems and Related Technology: Operational Practices, Costs, and 
Benefits." Proceedings of the International Conference of Enhanced Building Operations. LBNL Report 53406.

Neenan, B., D. Pratt, P. Cappers, J. Doane, J. Anderson, R. Boisvert, Goldman, C., O. Sezgen, G. Barbose, R. Bharvirkar, M. Kinter-Meyer, S. Shankle and D. Bates. 2003. "How and Why Customers Respond to Electricity Price Variability: A Study of NYISO and NYSERDA 2002 PRL Program Performance." LBNL-52209.

Nexant. 2002. “AB 29X and SB 5X Program Evaluation. First Quarter Report (January 1 to March 1, 2002)." Submitted to the California Energy Commission. Contract \#40000-070.

Nexant. 2001. “AB 979, AB 29X and SB 5X Peak Load Reduction Programs. December 2001 Annual Report." Submitted to the California Energy Commission and the California State Legislature.

Piette, M.A, S. Kinney, LBNL; M. Levi, D. McBride and S. May. 2002. "GEMnet Status and Accomplishments: GSA's Energy and Maintenance Network." Proceedings of the 2002 ACEEE Summer Study Proceedings. May 2002, LBNL-50733.

Piette, M.A., 1991. Controls to Reduce Electrical Peak Demands in Commercial Buildings. Center for the Analysis and Dissemination of Demonstrated Energy Technologies. CADDET Analyses Series 7, ISBN 90-72647-17-3. August 1991.

Shockman, C., O. Sezgen, N. Motegi, M.A. Piette, and D. Watson. 2004. "Market Transformation Lessons Learned from an Automated Demand Response Test in California." ACEEE Summer Study on Energy Efficiency in Buildings proceedings. August 2004.

Urban, G. L., and E von Hippel. 1988. "Lead User Analyses for the Development of New Industrial Products." Management Science volume 34, number 5 (May):569-82.

Webopedia. 2004. http://www.webopedia.com/TERM/X/XML.html. Accessed March $15,2004$. 


\section{Development and Evaluation of Fully Automated Demand Response In Large Facilities}

\section{APPENDICES}

March 30, 2004

Mary Ann Piette

Osman Sezgen

David S. Watson

Naoya Motegi

Lawrence Berkeley National Laboratory

Christine Shockman

Shockman Consulting

Laurie ten Hope

Program Manager, Energy Systems Integration

Sponsored by the California Energy Commission 

Table of Contents

Appendix I. Acronyms and Terminology ................................................................... I-1

Appendix II. Methodology - Additional Details..........................................................II-1

Appendix II-1. Whole-Building Level Method, Additional Detail.............................II-1

Appendix III. Sample Memorandum of Understanding .......................................... III-1

Appendix III-1. Project Participant Memorandum of Understanding .........................III-1

Appendix III-2. Automated Demand Response Test Questionnaire ……....................III-4

Appendix III-3. Time Schedule for Demand Response Test Participants ...................III-6

Appendix III-4. Price Signal for the Automated Demand Responses Tests ................III-7

Appendix III-5. RT Pricing Web Methods and XML Schema...................................III-10

Appendix IV. Participants List ................................................................................... IV-1

Appendix V. Site Questionnaires (Test 1, Test 2).........................................................V-1

Appendix V-1. Site Questionnaires - Albertsons ....................................................... V-1

Appendix V-2. Site Questionnaires - Bank of America............................................

Appendix V-3. Site Questionnaires - GSA Oakland.............................................. V-5

Appendix V-4. Site Questionnaires - Roche......................................................... V-8

Appendix V-5. Site Questionnaires - UCSB ....................................................... V-9

Appendix VI. Site Data Collection Points List ......................................................... VI-1

Appendix VI-1. Albertsons - Point List.......................................................... VI-1

Appendix VI-2. Bank of America - Point List .................................................... VI-1

Appendix VI-3. GSA Oakland - Point List.......................................................... VI-3

Appendix VI-4. Roche - Point List and Fan Spot Measurement Plan........................ VI-5

Appendix VI-5. UCSB - Shed Strategies and Point List .......................................... VI-9

Appendix VII. Additional Result Tables and Figures .............................................. VII-1

Appendix VII-1. First Day Test Results............................................................... VII-1

Appendix VII-2. B of A - Result Analysis by WebGen ......................................... VII-5

Appendix VII-3. GSA - Additional Figures...................................................... VII-7

Appendix VII-4. Roche - Additional Figures..................................................... VII-17

Appendix VII-5. UCSB - Additional Figures ………………………………...... VII-19

Appendix VIII. Previous DR Participation and Site Contact ................................ VIII-1

Appendix VIII-1.Prior Demand Response Program Participation ............................. VIII-1

Appendix VIII-2.Interaction with Site Contacts ..................................................... VIII-4

Appendix VIII-3.Problems Encountered During Test Period................................... VIII-8 



\section{Appendix I. Acronyms and Terminology}

Abstraction. In this document, the term "abstraction" is used with regard to translations between different communication protocols or I/O point mappings. In abstraction, details of one protocol or data format are added or removed in order to function properly in the other. Through translation and abstraction, low level protocols (such as EMCS protocols) can be changed into a common protocol or language (such as TCP/IP and XML). This process is often bi-directional (See translation, protocol, I/O, point mapping, EMCS, $T C P / I P$ and $X M L)$.

Auto-DR. Automated Demand Response

BACnet. An open data communication protocol for building automation and control networks. A data communication protocol is a set of rules governing the exchange of data over a computer network. The rules take the form of a written specification (in BACnet's case they are also on compact disk) that spells out what is required to conform to the protocol. BACnet was developed under the auspices of ASHRAE. The BACnet standard is also an ANSI standard (http://www.bacnet.org/FAQ/HPAC-3-97.html, BACnet.org 2004).

BACnet MSTP. Short for BACnet Master/Slave Twisted Pair. BACnet MSTP describes the characteristics of the BACnet communication protocol when used in a Master/Slave architecture over twisted pair wiring.

Bandwidth. The amount of data that can be transmitted in a fixed amount of time. For digital devices, the bandwidth is usually expressed in bits per second (bps) or bytes per second. For analog devices, the bandwidth is expressed in cycles per second, or Hertz (Hz) (Webopedia, 2004).

Business Logic. In the Auto-DR tests, business logic determines the EMCS actions to be implemented based on price and business rules.

Client (computer). The client part of a client-server architecture. Typically, a client is an application that runs on a personal computer or workstation and relies on a server to perform the operations requested by the client. For example, an e-mail client is an application that enables you to send and receive e-mail. In the Auto-DR tests the clients at each site polled the server to get current pricing information.

Co-lo. See Co-Location.

Co-Location. A server, usually a Web server, that is located at a dedicated facility designed with resources which include a secured cage or cabinet, regulated power, HVAC sufficient to cool all the electronic equipment, dedicated Internet connection, security and support. These co-location facilities offer their customers a secure place to physically house their hardware and equipment as opposed to locating it in their offices or warehouse where the potential for fire, theft or vandalism is much greater. Most co- 
location facilities offer high-security, including cameras, fire detection and extinguishing devices, multiple connection feeds, filtered power, backup power generators and other features to ensure a level of high-availability which is mandatory for all Web-based, virtual businesses. Co-location sites are being built at various points around the world to provide services to the rapidly expanding Web hosting and e-commerce marketplace. The term co-location is also known as co-lo (Webopedia, 2004).

Control network. A network of controllers, data gathering panels and other devices that measure values from sensors and send commands to actuators. Control networks have been designed and optimized for the requirements of these systems including low installed cost and small communication packet sizes. Historically, many control networks have been based on RS-485 communications using proprietary protocols. Increasingly open protocols are being used including BACnet and LonTalk over RS-485 and Internet Protocols (IP). Control networks are generally separate from enterprise networks.

Data logging. The process by which data from I/O points are logged into a database.

Dewpoint. The temperature at which water vapor is saturated.

Digital outputs (DO). In an I/O controller, digital outputs are used to command equipment on or off. Physically, a digital output consists of an automatically controlled relay contact. Constant volume fans and pumps and lights can be commanded on or off with a digital output (see $I / O$ controller).

DMZ. Short for demilitarized zone, a computer or small subnetwork that sits between a trusted internal network, such as a corporate private LAN, and an untrusted external network, such as the public Internet. Typically, the DMZ contains devices accessible to Internet traffic, such as Web servers. The term comes from military use, meaning a buffer area between two enemies (Webopedia, 2004).

DR. Demand Response.

EIS. Energy Information System.

Embedded devices. Special purpose computers with the following attributes:

1) Targeted functionality with little, if any, flexibility for the user to add different programs or customize the device.

2) User interfaces are usually limited to allow targeted functionality only. May include small LCD screens, LEDs, buttons switches and knobs. QWERTY keyboards and Cathode Ray Tube display screens are generally not included.

3) Memory is usually cost optimized for the targeted functionality. Read only memory (ROM) and flash memory chips are usually used in lieu drives with spinning disks. 
4) Form factor is specially designed for the targeted functionality. Examples of embedded devices include Internet routers, automotive engine computers and cell phones.

5) Often designed for reliable continuous operation with little or no human interaction.

EMCS. Energy Management and Control System.

Enterprise. A business organization. In the computer industry, the term is often used to describe any large organization that utilizes computers. An intranet is an example of an enterprise computing system (Webopedia, 2004).

Ethernet. A local-area network (LAN) architecture developed by Xerox Corporation in cooperation with DEC and Intel in 1976. Ethernet uses a bus or star topology and supports data transfer rates of $10 \mathrm{Mbps}$. The Ethernet specification served as the basis for the IEEE 802.3 standard, which specifies the physical and lower software layers. Ethernet uses the CSMA/CD access method to handle simultaneous demands. It is one of the most widely implemented LAN standards. A newer version of Ethernet, called 100Base-T (or Fast Ethernet), supports data transfer rates of $100 \mathrm{Mbps}$. The newest version, Gigabit Ethernet supports data rates of 1 gigabit (1,000 megabits) per second (Webopedia, 2004)

Firewall. A system designed to prevent unauthorized access to or from a private network. Firewalls can be implemented in either or both hardware and software. Firewalls are frequently used to prevent unauthorized Internet users from accessing private networks connected to the Internet, especially intranets. All messages entering or leaving the intranet pass through the firewall, which examines each message and blocks those that do not meet the specified security criteria. There are several types of firewall techniques:

1) Packet filter: Looks at each packet entering or leaving the network and accepts or rejects it based on user-defined rules. Packet filtering is fairly effective and transparent to users, but it is difficult to configure. In addition, it is susceptible to IP spoofing.

2) Application gateway: Applies security mechanisms to specific applications, such as FTP and Telnet servers. This is very effective, but can impose performance degradations.

3) Circuit-level gateway: Applies security mechanisms when a TCP or UDP connection is established. Once the connection has been made, packets can flow between the hosts without further checking.

4) Proxy server: Intercepts all messages entering and leaving the network. The proxy server effectively hides the true network addresses.

In practice, many firewalls use two or more of these techniques in concert. A firewall is considered a first line of defense in protecting private information. For greater security, data can be encrypted (Webopedia, 2004). 
Fully-Automated Demand Response. Fully-Automated Demand Response is initiated at a building or facility through receipt of an external communications signal - facility staff set up a pre-programmed load shedding strategy which is automatically initiated by the system without the need for human intervention.

Gateway. Gateways used in building telemetry systems provide several functions. First, they connect two otherwise incompatible networks (i.e., networks with different protocols) and allow communications between them. Second, they provide translation and usually abstraction of messages passed between two networks. Third, they often provide other features such as data logging, and control and monitoring of $\mathrm{I} / \mathrm{O}$ points.

Generation. In electronics, computer equipment and software, the term "generation" is used to describe a major upgrade for which previous versions may or may not be compatible.

High Availability. Used to quantify the "uptime" for computer servers and systems. High availability is a requirement for operation of mission critical systems. High availability systems are often described in terms of the number of "nines" of availability (i.e., four $9 \mathrm{~s}$ or $99.99 \%$ means less than one hour of unscheduled downtime per year).

HTTP. Short for HyperText Transfer Protocol, the underlying protocol used by the World Wide Web. HTTP defines how messages are formatted and transmitted, and what actions Web servers and browsers should take in response to various commands. For example, when you enter a URL in your browser, this actually sends an HTTP command to the Web server directing it to fetch and transmit the requested Web page (Webopedia, 2004).

Human Machine Interface (HMI). Nowadays humans interact more with computerbased technology than with hammers and drills. Unlike tools, the visible shape and controls of a computer do not communicate its purpose. The task of an HMI is to make the function of a technology self-evident. Much like a well-designed hammer fits the user's hand and makes a physical task easy; a well-designed HMI must fit the user's mental map of the task he or she wishes to carry out (International Engineering Consortium (www.iec.org), 2004). In control systems, the HMI usually takes the form of a computer display screen with specially designed graphical representations of the mechanical systems or processes including real-time data from sensors.

HVAC. Heating, Ventilation, and Air Conditioning.

I/O. Abbreviation for Input/Output. Commonly used in the controls industry. Refers to inputs (such as sensors) and outputs (such as actuators) (see abstraction, point mapping and translation).

I/O controller. A device that measures input values from sensors and commands outputs such as temperature control valves, usually to maintain a defined setpoint. An I/O controller has the internal intelligence to perform local control. 
I/O module. A device that measures input values from sensors and commands outputs such as temperature control valves based on instructions from a remote controller. An I/O module usually does not have the internal intelligence to perform local control.

Internet. A global network connecting millions of computers. More than 100 countries are linked into exchanges of data, news and opinions. Unlike online services, which are centrally controlled, the Internet is decentralized by design. Each Internet computer, called a host, is independent. Its operators can choose which Internet services to use and which local services to make available to the global Internet community. Remarkably, this anarchy by design works exceedingly well. There are a variety of ways to access the Internet, including through online services, such as America Online, and commercial Internet Service Providers (ISP) (Webopedia, 2004). References to the public Internet, as described here, are spelled "Internet" (capital "I"). The lesser used term, "internet" (lower case "i"), refers to a network of intranets that are usually privately operated (see intranet).

Intranet. A network based on TCP/IP protocols (an internet) belonging to an organization, usually a corporation, accessible only by the organization's members, employees, or others with authorization. An intranet's Web sites look and act just like any other Web sites, but the firewall surrounding an intranet fends off unauthorized access. Like the Internet itself, intranets are used to share information (Webopedia, 2004).

IP I/O device. A device that measures inputs (e.g., electric meter data) and controls outputs (e.g., relays) that can be measured and actuated remotely over a LAN, WAN or Internet using Internet Protocols (IP).

IP relay. A device with a relay or relays that can be actuated remotely over a LAN, WAN or Internet using Internet Protocols (IP).

ISO. Independent System Operator.

IT. Short for Information Technology, and pronounced as separate letters, the broad subject concerned with all aspects of managing and processing information, especially within a large organization or company. Because computers are central to information management, computer departments within companies and universities are often called IT departments. Some companies refer to this department as IS (Information Services) or MIS (Management Information Services) (Webopedia, 2004).

LAN. A computer network that spans a relatively small area. Most LANs are confined to a single building or group of buildings. Most LANs connect workstations and personal computers. Each node (individual computer) in a LAN has its own central processing unit (CPU) with which it executes programs, but it also is able to access data and devices anywhere on the LAN. This means that many users can share devices, such as laser printers, as well as data. Users can also use the LAN to communicate with each other 
such as by sending e-mail. There are many different types of LANs with Ethernets being the most common for PCs (Webopedia, 2004).

Load balancing. Distributing processing and communications activity evenly across a computer network so that no single device is overwhelmed. Load balancing is especially important for networks where it's difficult to predict the number of requests that will be issued to a server. Busy Web sites typically employ two or more Web servers in a load balancing scheme. If one server starts to get swamped, requests are forwarded to another server with more capacity. Load balancing can also refer to the communications channels themselves (Webopedia, 2004).

LonTalk ${ }^{\mathrm{TM}}$. An open communications protocol used in building control systems and other industries. Publicly published under ANSI/ EIA -709.1 - (American National Standards Institute/Electronic Industries Alliance). Since 1999, LonTalk protocol may be implemented on any microprocessor.

LonWorks ${ }^{\mathrm{TM}}$. Control and communication products available from Echelon Corporation and other companies using Echelon products. LonWorks products use LonTalk open protocol for communications (see LonTalk).

Machine-to-Machine (M2M). Machine to Machine (M2M) is a term used to describe the technologies that enable computers, embedded processors, smart sensors, actuators and mobile devices to communicate with one another, take measurements and make decisions - often without human intervention.

Manual Demand Response. Manual demand response involves manually turning off lights or equipment; this can be a labor-intensive approach.

MCC. Motor Control Center.

Modbus. An open standard control network protocol originally developed by Modicon, a simple master/slave protocol over RS-485. Current protocol also supports TCP/IP over an Ethernet. Modbus is a common interface with electrical equipment such as meters and generators. It is not typically used for whole building EMCSs.

Modem. A hardware device that allows computers to communicate with one another over any phone-based network.

NOC. Short for network operations center, the physical space from which a typically large telecommunications network is managed, monitored and supervised. The NOC coordinates network troubles; provides problem management and router configuration services; manages network changes; allocates and manages domain names and IP addresses; monitors routers, switches, hubs and UPS systems that keep the network operating smoothly; and manages the distribution and updating of software and coordinates with affiliated networks. NOCs also provide network accessibility to users 
connecting to the network from outside of the physical office space or campus (Webopedia, 2004).

OAT. Outside air temperature.

oBIX. Open Building Information eXchange is an industry-wide initiative to define open XML- and Web services-based mechanisms for building control systems.

ODBC. Short for Open DataBase Connectivity, a standard database access method developed by Microsoft Corporation. The goal of ODBC is to make it possible to access any data from any application, regardless of which database management system (DBMS) is handling the data. ODBC manages this by inserting a middle layer, called a database driver, between an application and the DBMS. The purpose of this layer is to translate the application's data queries into commands that the DBMS understands. For this to work, both the application and the DBMS must be ODBC-compliant -- that is, the application must be capable of issuing ODBC commands and the DBMS must be capable of responding to them. Since version 2.0, the standard supports SAG SQL (Webopedia, 2004).

Onboard. Refers to electronic components that are mounted on the main printed circuit board as opposed to components that are mounted remotely and connected via wires.

Open protocol. A communications protocol that is used to communicate between devices of any compliant manufacturer or organization. Open protocols are published in a public forum for use by all interested parties (see proprietary protocol).

Point mapping. The process by which I/O points are mapped to another system or protocol (see abstraction, I/O and translation).

Point of sale (POS). Refers to all components in the POS system including bar code scanners, cash registers, printers and all communications equipment that is used to transfer information about the sale of each item back to a central database. At some sites in the Auto-DR tests, the point of sale communications infrastructure was used to command the shed of electric loads based on electricity pricing information.

Poll. A method by which one computer requests information from another.

Polling Client. In the Auto-DR tests, the polling client is the software used to poll the server to get the price signal.

Price Server. In the Auto-DR tests, the price server is the common source for the current price information.

Proprietary protocol. A communications protocol that is used to communicate between devices of one manufacturer or organization while effectively disallowing all other 
devices to exist on the same network. Proprietary protocols are not published in a public forum (see open protocol).

Protocol (data communication). A data communication protocol is a set of rules governing the exchange of data over a computer network.

Public Switched Telephone Network (PSTN). International telephone system based on copper wires carrying analog voice data. This is in contrast to newer telephone networks based on digital technologies (Webopedia 2004).

Pull architecture. In a client-server architecture, the client "pulls" information from the server by polling (see poll).

Pulse. Contact closures that are measured by an I/O device. Pulses are often produced by electric meters and other devices to indicate a given unit of measurement (for example, 1 pulse $=1 \mathrm{kWh}$ ).

Real-time. In real-time control and monitoring systems, data is measured, displayed and controlled at a rate fast enough that the system latencies are negligible compared with the process at hand. Acceptable latency can vary substantially based on the type of process (for example, from 1 millisecond to several minutes).

Semi-Automated Response. Semi-Automated Response involves the use of building energy management control systems for load shedding, where a pre-programmed load shedding strategy is initiated by facilities staff.

Sensor. a device that responds to a physical stimulus (heat, light, sound, pressure, magnetism, or a particular motion) and transmits a resulting impulse that can be used for measurement or operating a controller (Merriam-Webster Online Dictionary, 2004).

Server (computer). Servers are often dedicated, meaning that they perform no other tasks besides their server tasks. On multiprocessing operating systems, however, a single computer can execute several programs at once. A server in this case could refer to the program that is managing resources rather than the entire computer. In the 2003 AutoDR tests, pricing information was "served" from a Web services server hosted by Infotility, Inc.

Setpoint. The target value that an I/O controller attempts to maintain. Setpoint values (for example, temperature or pressure) are maintained through adjustments of the final control elements (temperature control valves, dampers, etc.).

SIMM. Acronym for single in-line memory module, a small circuit board that can hold a group of memory chips. Typically, SIMMs hold up to eight (on Macintoshes) or nine (on PCs) RAM chips. On PCs, the ninth chip is often used for parity error checking. Unlike memory chips, SIMMs are measured in bytes rather than bits. SIMMs are easier to install than individual memory chips. The bus from a SIMM to the actual memory chips is 32 
bits wide. A newer technology, called dual in-line memory module (DIMM), provides a 64-bit bus. For modern Pentium microprocessors that have a 64-bit bus, you must use either DIMMs or pairs of SIMMs (Webopedia, 2004)

SOAP. Short for Simple Object Access Protocol, a lightweight XML-based messaging protocol used to encode the information in Web service request and response messages before sending them over a network. SOAP messages are independent of any operating system or protocol and may be transported using a variety of Internet protocols, including SMTP, MIME, and HTTP (Webopedia, 2004).

Systems Integrator. An individual or company that specializes in building complete computer systems by putting together components from different vendors. Unlike software developers, systems integrators typically do not produce any original code. Instead they enable a company to use off-the-shelf hardware and software packages to meet the company's computing needs (Webopedia, 2004).

TCP/IP (Transmission Control Protocol / Internet Protocol). Internet Protocol that specifies the format of packets and the addressing scheme. Most networks combine IP with a higher-level protocol called Transmission Control Protocol (TCP), which establishes a virtual connection between a destination and a source.

Telemetry. A communications process that enables monitoring and/or control of remote or inaccessible sensors and actuators. Telemetry often uses radio frequency signals or Internet technologies for communications.

Time stamp. A digital message that indicates the time in which a given computer transaction occurred. The time stamp message is usually associated with and stored with the original transaction record. Time stamps are also to note when data is logged or stored.

Translation. The process by which I/O points are translated to another system or protocol. Translation changes messages in one protocol to the same messages in another (see abstraction, I/O and point mapping).

UTC. "Coordinated Universal Time" (abbreviated UTC), is the basis for the worldwide system of civil time. This time scale is kept by time laboratories around the world, including the U.S. Naval Observatory, and is determined using highly precise atomic clocks (U.S. Naval Observatory 2004 http://aa.usno.navy.mil/faq/docs/UT.html). UTC differs from Greenwich Mean Time (GMT) in that GMT is based on the rotation of the Earth which is substantially less accurate than atomic clocks. When GMT differs from UTC by more than 0.9 seconds, UTC is re-calibrated by adding a "leap second" so that it is closer to the (less precise and uncontrollable) rotation of the Earth.

VPN. Short for virtual private network, a network that is constructed by using public wires to connect nodes. For example, there are a number of systems that enable you to create networks using the Internet as the medium for transporting data. These systems use 
encryption and other security mechanisms to ensure that only authorized users can access the network and that the data cannot be intercepted (Webopedia, 2004).

WAN (Wide Area Network). A computer network that spans a relatively large geographical area. Typically, a WAN consists of two or more local-area networks (LANs). The largest WAN in existence is the Internet, which is open to the public. Private and corporate WANs use dedicated leased lines or other means of assuring that the network is only available to authorized users of the organization (Webopedia, 2004).

WBP. Whole building power.

Web Services. The infrastructure of the Auto-DR System is based on a set of technologies known as Web Services. Web Services have emerged as an important new type of application used in creating distributed computing solutions across the Internet. Properly designed Web services are completely independent of computer platform (Microsoft, Linux, Unix, Mac, etc.). Web pages are accessed by people to view information on the Internet. Web services are used by computers to share information on the Internet. Since human intervention is not required, this technology is sometimes referred to as "Machine-to-Machine" or "M2M". XML is often used to enable Web services. M2M is a superset of technologies that includes some XML/Web services based systems (see XML, Machine to Machine).

XML (Extensible Markup Language). XML is a "meta-language", a language for describing other languages that allows the design of customized markup languages for different types of documents on the Web (Flynn, 2003). It allows designers to create their own customized tags, enabling the definition, transmission, validation, and interpretation of data between applications and between organizations (Webopedia, 2004). 


\section{Appendix II. Methodology - Additional Details}

\section{Appendix II-1. Whole-Building Level Method, Additional Detail}

Outside air temperature (OAT) regression models were developed for each fifteen minute time interval (See Figure III-2 for some of the regression models generated for the GSA data). A total of 96 models were developed for one test day. For example, the whole building power and outside air temperature (OAT) at 1:15 p.m. on the previous days' are used as input data to develop a regression model for 1:15 p.m. on the test day. To estimate the shed-day baseline power $[\mathrm{kW}]$ at 1:15 pm, OAT trend data at 1:15 pm of the shed-day is put into the regression model equation. The whole building power baseline load shape is developed by doing this for each 15-minute interval.

Indication from the regression models are;

- Right-up slope indicates that whole building power (WBP) increases as OAT rises.

- Steeper slope indicates stronger correlation between WBP and OAT.

- Flat slope indicates WBP is not significantly affected by OAT.

- The width of the data range in WBP indicates the degree of errors. Wider width ranges indicates larger errors.

The lines generated using the regression models for the time intervals during operating hours (9:00, 12:00 and 15:00) have steep slopes, indicating that WBP has a strong correlation with OAT. On the other hand, the models at night have flat slopes. Nighttime operation is constant and not significantly affected by OAT. 


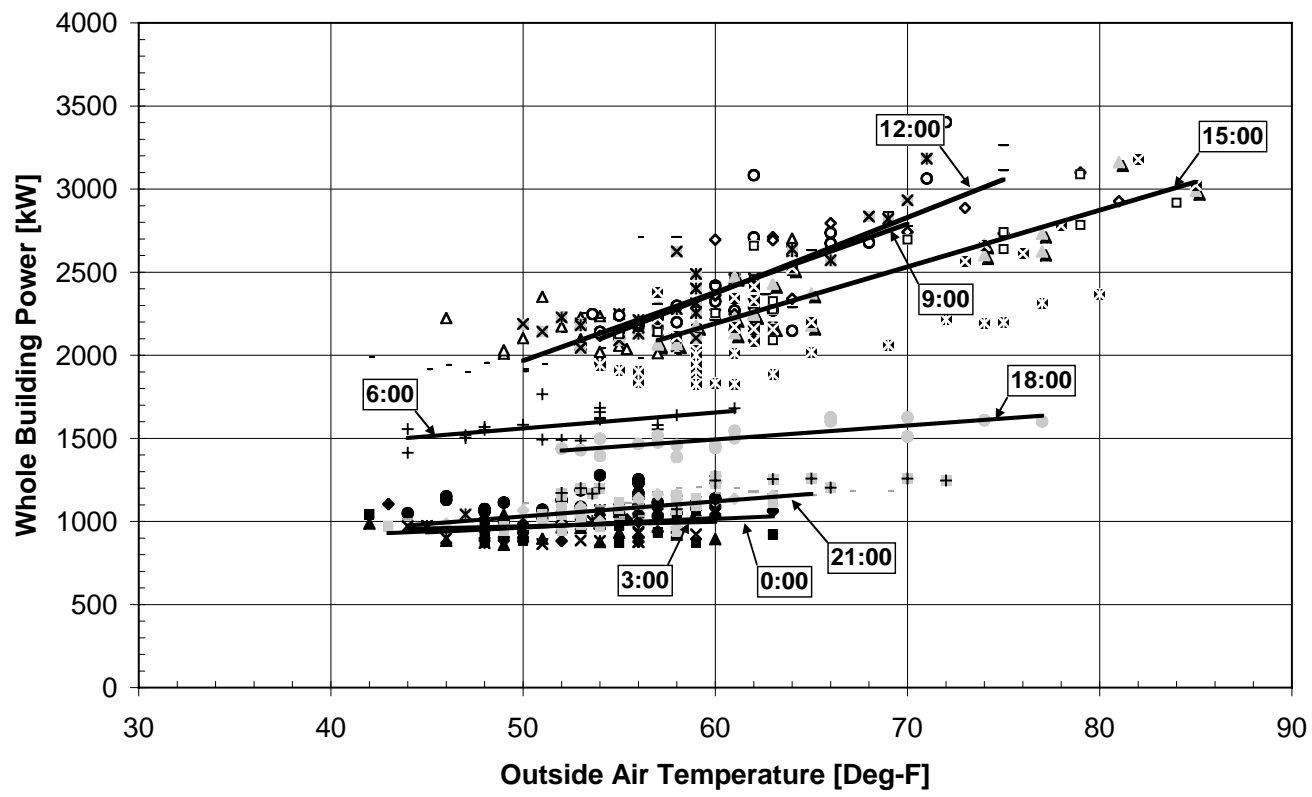

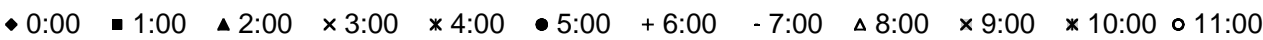

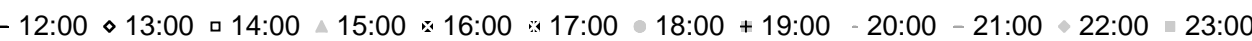

Figure II-1. OAT Linear Regression Model for A Sample of the 96 Fifteen Minute Time Intervals (GSA) 


\title{
Appendix III. Sample Memorandum of Understanding
}

\section{Appendix III-1. Project Participant Memorandum of Understanding}

\author{
Project Participant Memorandum of Understanding \\ Between \\ Environmental Energy Technologies Division, \\ Lawrence Berkeley National Laboratory (LBNL) \\ and \\ Demand Responsive Technology Demonstration
}

August 27, 2003

\section{Introduction}

LBNL has developed a research project for the California Energy Commission to test automated Demand Response (DR) technologies in buildings. This Memorandum of Understanding (MOU) provides a brief description of the objectives of the DR project, what LBNL plans to undertake and what is expected of the Participant.

\section{Project Objectives}

The objectives of this research project are:

- to improve understanding of the status of automated demand responsive building systems, particularly the levels of automation in best practices

- to quantify demand-savings potential of automated demand responsive systems

- to identify technology gaps and priorities to improve future systems

- to understand key features of the market for DR systems and decision making perspectives about the adoption of DR technology

- to develop and test an automated signal to initiate demand response events

\section{Collection of Information on DR System}

LBNL has selected site name (city, state) to participate in the DR Demonstration project because of the State-Of-the-Art building control technology at the site. LBNL will collect and compile the following type of information to include but is not limited to:

- Site characteristics (size, type, location, HVAC systems, etc.)

- DR-Systems: software, firmware, and hardware, etc., installed at the site.

- Monitoring, control, and reporting attributes of the system

- Level of automation, human expertise and experience required

- DR-System and Energy Management capabilities and strategies used: How is the DR-system used to optimize energy performance, shed, or shift demand? 
- Document first costs for technologies that facilitates the automated demandresponse, including capital and installation costs

- Estimate operating costs, including maintenance and support costs: How does the DR enabling technology change operating costs?

- Determine peak demand and energy savings: How does the DR technology increase flexibility of the facility and therefore increase savings in energy expenditures?

The "Automated Demand Response Test Site Questionnaire" dated August 5, 2003 is to be completed and returned to Dave Watson (dswatson@lbl.gov) by August 15, 2003. Owner/operators will coordinate this effort with their Energy Information System suppliers. Dave Watson is available at 5104865562.

The Participant agrees to provide the above information to LBNL. Much of the information will be included in a public report from LBNL to the CEC. Upon Participant's advance request, LBNL will provide a copy of the report to Participant prior to making such report public.

\section{Demand Response Test}

All participants are responsible for reviewing and meeting the attached "Time Schedule for Demand Response Test Participants" dated August 5, 2003.

During late summer 2003 LBNL will send the Participant an XML signal via the internet that contains information to represent electricity prices.

The Participant will work with their controls and DR system vendor and in house staff to modify their system to be able receive or retrieve the XML signal, send back an acknowledgement, and initiate an automated shed. The tests will take place during a 2 week period in late September or October 2003. The automated response will not be requested during more than two working days. These days may be non-consecutive. Within a test day, response will not be requested for more than 3 hours. The Participant will be able to override the test if need be. However, LBNL would like to verify that the shed was fully automated with no operator intervention.

Further definition of the price signal is provided in two documents "Price Signal for the Automated Demand Responses Tests" (dated June 26, 2003) and "RT Pricing Web Methods and XML Schema" (dated August 4, 2003, revised from the July 2, 2003 RT Pricing Web Methods and XML Schema document), which are attached. The baseline price for no action will be 10 cents/kWh. The first level of price increase will be to 30 cents/kWh. The second level will be 75 cents/kWh. Triggers for the automated shed should be based on those prices.

LBNL plans to compile HVAC, control, communications, energy, and other building time series data during the test to evaluate the shed. The development of this information to evaluate the success of the automated shedding will require additional collaboration 
between LBNL, the building owner/operator, and the EIS provider. Time for this collaboration effort should be anticipated. LBNL plans to report on the results of the shed in a report to the CEC. Results from the Participant will be compared with results from other sites as well.

\section{Terms of Agreement}

LBNL is in no way responsible for any issues that arise at the building facility as a result of the tests. LBNL understands that due to circumstances that cannot be predicted, the Participant may not be able to complete their participation in the project. LBNL would like to be informed of such a decision at least one month in advance of the test.

This memorandum is intended to memorialize the understanding of LBNL and Participant in the research of automated DR in buildings. The parties agree that this memorandum is not intended to be legally binding and that if the parties desire to create specific, legally-binding obligations, such binding obligations shall only arise under a separate written agreement signed by duly authorized representatives of both parties.

Signed:

Mary Ann Piette

Staff Scientist, and Deputy Group Leader

Lawrence Berkeley National Laboratory

Signature:

Date:

Contact Name

$\underline{\text { Title }}$

Organization

Signature:

Date:

Attachments (Reference Documents)

- Automated Demand Response Test Site Questionnaire - Updated August 5, 2003

- Time Schedule for Demand Response Test Participants - Updated August 5, 2003

- Price Signal for the Automated Demand Responses Tests - Dated June 26, 2003

- $\quad$ RT Pricing Web Methods and XML Schema - Updated August 4, 2003 


\section{Appendix III-2. Automated Demand Response Test Questionnaire Automated Demand Response Test-Site Questionnaire Updated August 5, 2003}

1) What is the vendor \& system type of the EMCS? (e.g. Johnson MetaSys)

2) What protocols are supported by the EMCS? (e.g. N2, BACnet, LON, Proprietary)

3) What is the gateway vendor that connects EMCS to the remote access network? (e.g. same vendor as EMCS, Silicon Energy, Envenergy etc.)

4) Can the gateway export meter-data using http (port 80) and can it format them in XML?

5) Can the gateway exports the data to a third party server?

6) How does the gateway communicate to the equipment or automation system? What is the protocol used to communicate (BACNET, LON. MODBUS)?

7) Who was the installing contractor for the DR system? (e.g. the EMCS vendor, the gateway vendor, other)

8) Describe the remote access network: (e.g. Corporate LAN/WAN, T1, DSL, Cable modem, POTS/PSTN modem (dial-up), pager network, other). How does the gateway communicate back to the host or central server (TCP/IP, proprietary)?

9) In addition to Demand Response capability, does the system provide:

- Real-time Remote monitoring of EMCS?

- Real-time monitoring of revenue meters and other sub-meters?

- Remote control of EMCS (e.g. adjust setpoints)?

- Remote alarm annunciation (text messaging, pagers, email)?

- Trended data viewing \& downloading? How is data being logged?

10) What is the location of the device that will receive the XML signal (on-site, off site)?

11) What device will be receiving the XML signal (gateway, central server, other device)?

12) To receive the XML signal, the device must have a public IP address. If the device is behind a firewall, what TCP/IP ports are accessible?

13) If the system features remote web access, what is the location of the web server (onsite, off-site)? 
14) Describe the amount of labor necessary to revise the system to interface with the defined standard XML signal (do not include logic changes to EMCS for load shedding).

15) On the LAN where the gateway is installed, which ports are open (in, out) on the firewall?

16) On the LAN where the device receiving price signal is installed, which ports are open (in, out) on the firewall?

17) Describe the system cost including hardware, software, licensing and initial installation fees, annual or monthly fees, and service or maintenance fees. 


\section{Appendix III-3. Time Schedule for Demand Response Test Participants}

\begin{tabular}{|l|c|l|}
\hline \multicolumn{3}{|c|}{ Time Schedule for Demand Response Test Participants } \\
(Dated - August 5, 2003)
\end{tabular}




\title{
Appendix III-4. Price Signal for the Automated Demand Responses Tests
}

\author{
MEMO: Price Signal for the Automated Demand Response Tests
}

\section{FROM: Osman Sezgen and Mary Ann Piette Lawrence Berkeley National Laboratory \\ TO: $\quad$ Participating Facilities}

\section{DATE: June 26, 2003}

This memo provides an overview of the price signals for the automated Demand Response (DR) test.

The automated DR tests will be initiated by an electricity price signal sent from Infotility using "push" architecture. [Push architecture will be used if the technology used by the EMS can support it or can integrate it. If not, "pull" architecture will be used--the EMS queries a server that returns the real time price.] The profile of the price-signal will be determined and controlled by the Lawrence Berkeley National Laboratory as further described below.

We request that each site acknowledges the receipt of the price data as further described below. Following receipt of the signal we request that your system automatically initiates your load shedding response when the price signal moves to a level above standard timeof-use pricing. We will discuss this with you.

The signal itself will be simple and the profile of the electric prices will be designed to cause minimal inconvenience to the facilities that are participating in the DR test.

\section{Description of the Price-Signal (Hardware/Software Point of View)}

The price signal will be broadcasted every 15 minutes (approximately on the hour, and every fifteen minutes after the hour). The price broadcasted will be in effect in about 15 minutes. (The facility will have at least 15 minutes of advance notice before the price changes.) Every signal will have two time stamps: (1) the time that it is broadcasted, and (2) the time that it will be in effect. The time that the signal is broadcasted may be slightly earlier than the 15-minute notice period but the times of effectiveness of prices will be on discrete times (on the hour and every fifteen minutes after the hour). The time that it will be in effect is a 15 min ending time.

The communications systems at the facility will need to be programmed to accept the electric price signals from Infotility and send an acknowledgement signal back. This acknowledgement signal should include the full information about the original signal including the time stamps.

The facility will need to provide an IP address to Infotility. This address is going to be used as the target for the price information. To be able to receive price information pushed by Infotility, there are 2 options: 
1. "Push" architecture: The EMS of the facility will have to integrate a "connector" that communicates with the Infotility servers in real time ("Push" architecture).

2. "Pull" architecture: actually, the "pull" architecture includes both "push" and "pull" technologies: an application from Infotility would be installed on a computer on the LAN of the facility. Infotility's servers would push real time pricing to this application. The EMS of the facility would pull the real time prices from this application.

\section{Description of the Price-Signal (Operations Point of View)}

Although the signal is designed to change every fifteen minutes, the actual signal will be constrained to make it less onerous to the participating facilities. The objectives are:

- Give some predictability on the frequency side (frequency of price changes)

- Give some predictability on the price side (level of prices)

- Give some predictability on the duration of the high prices

The shape of the signal will be such that:

- When the price level changes, the level will stay the same for at least one full hour.

- There will be three levels of price: normal (10 cents/kWh), medium (30cents/kWh) and high ( 75 cents/kWh), the facility operator can preprogram response actions for each level.

- The duration of price changes to higher than normal will not exceed 3 hours (thus shorter than CPP) and prices will move above normal only once during one day (once prices move, the facility can be sure that it will be back to normal within 3 hours and will not move again for the day).

- Signals may change prices between the hours $12 \mathrm{pm}$ to $7 \mathrm{pm}$ (weekdays). The latest signal that may change prices will be for $6 \mathrm{pm}$ and will change the price effective between $6 \mathrm{pm}$ to $7 \mathrm{pm}$.

- The tests will take place within 2 weeks and we will not change prices during more than two working days within this period. Thus, we want all of the communication systems to be ready for the test at the same time. The test is intended to take place at all 6 sites in real time.

For example, as shown in Figure 1 if we would like to change the price to a medium level at $12 \mathrm{pm}$, we will send a signal by 11:45 am. Once we set the price for $12 \mathrm{pm}$, we will not change it until $1 \mathrm{pm}$. If we want to change the price at $1 \mathrm{pm}$ (to high or normal), we will send another signal by $12: 45 \mathrm{pm}$. If we change the price to medium at 12pm and high at $1 \mathrm{pm}$, we can only hold the prices above normal until $3 \mathrm{pm}$. If we set the price to medium at $12 \mathrm{pm}$ and set it back to normal at $1 \mathrm{pm}$, we will not change the price level again until possibly the next weekday. 
Finally, the facility will be able to opt out within the 15-minute notice period just before a higher price comes into effect. (During the time period between when the signal is sent and when the price is in effect, the facility can inform us and opt out of the test.)

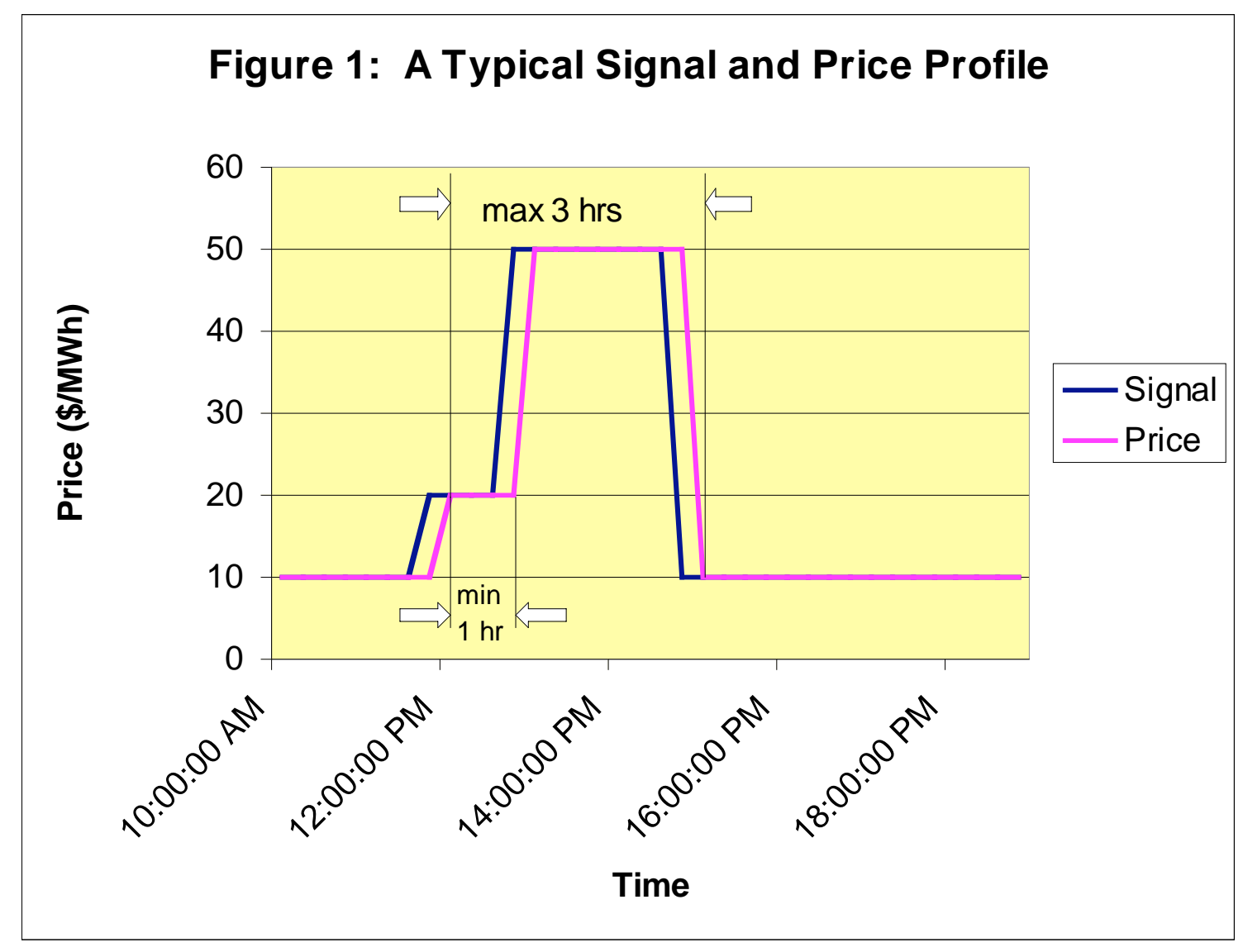




\section{Appendix III-5. RT Pricing Web Methods and XML Schema RT Pricing: Web Methods and XML Schema}

Author: Nicolas Kardas (Infotility) with additions from Mary Ann Piette (LBNL) Date: August $4^{\text {th }}, 2003$

\section{Introduction:}

Infotility will deliver an electricity price stream to the facilities in the automated demand response research project developed by Lawrence Berkeley National Laboratory. The client applications connected to the LAN at the user's site can receive the electricity price stream through a variety of methods. The client application may direct the price stream directly to an Energy Management Control System (EMCS), an electric meter, an Energy Information System (EIS) gateway device or any a software application. These options are shown in Figure 1. Based on previous discussions with the 6 sites, we believe that most sites will received the information through and EIS or related EMCS application.

Note: a client application may not be needed: the EMCS/EIS can directly call the web services.

Figure 1. Real-time Pricing Delivery Service Example

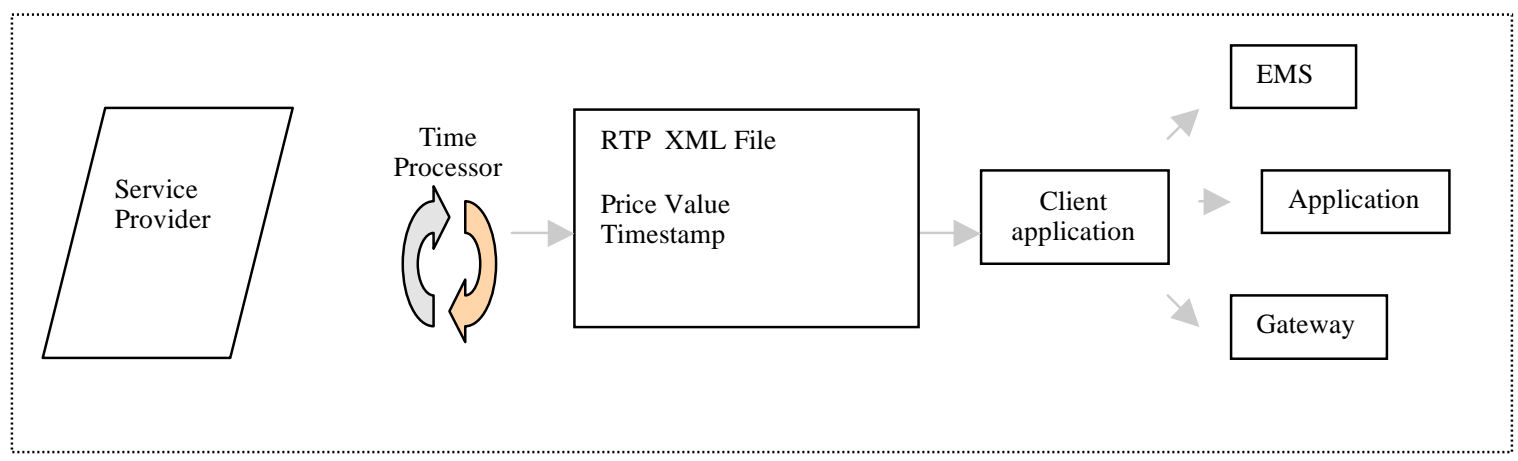

In order to get access to the prices, there are 2 options:

1. Option 1: The End-users regularly call a Web service that returns the prices. This would be done automatically by the client application or the EMCS/EIS. The prices are formatted using the XML RT Pricing schema (see below).

2. Option 2: The client application or the EMCS integrates a component that automatically receives the new prices when they are available on the server. This requires more integration work than option 1 .

Option 1 is certainly the easiest implementation since it relies on Web services to pass the prices to the client applications. EMS/EIS may already have connectors available to call Web services methods.

The project team and the participants have considered both options and have decided to use only the option 1 for this project. 


\section{The server will check that the price are successfully received by the participants EIS}

In order to give to the project team visibility regarding which participants get the price, when they get it and if they get it right, the servers logs each web service transaction between the server and the participant EIS and checks that the prices are received correctly by the participants EIS.

To be able to perform this check, when a participant EIS calls a web service to get a new price, it has to pass, as a parameter, the value of the last price it received from the server and the timestamp of this price. The server will log this data and will check that the price received by the participant EIS is the same as the one that was returned by the server.

If a participant EIS has not called the web service for more than $\mathrm{x}$ minutes or if the price was not successfully received by the participant, an Email alert will be sent to the participant and the project team.

\section{Web methods to call to get Real Time Prices}

They are 2 steps to get a real time price from Infotility's servers:

- Step 1: Login to the system

- Step 2: Call a Web method to get the real time prices

3.1. Web method to login

The signature of the Web method is the following:

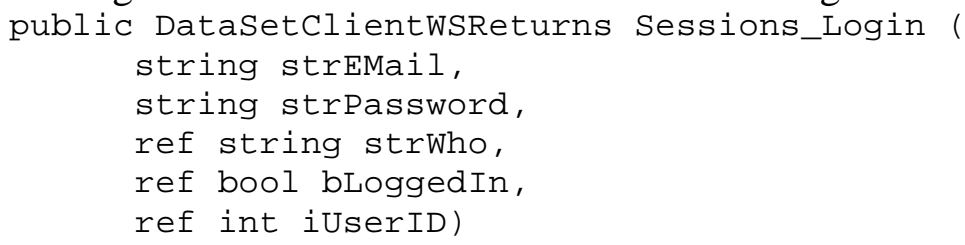

This method must be called only once when the application starts. The Email and Password are passed to uniquely identify the user in the system. The method returns 3 parameters:

- strWho: it is a unique key that identifies the session that has been created for the user in the system. This parameter is returned by the server. This parameter has to be passed in each web method call to get the price data.

- bLoggedIn: it is equal to true if the login has been successful on the server

- iUserID: the userID of the user in the system.

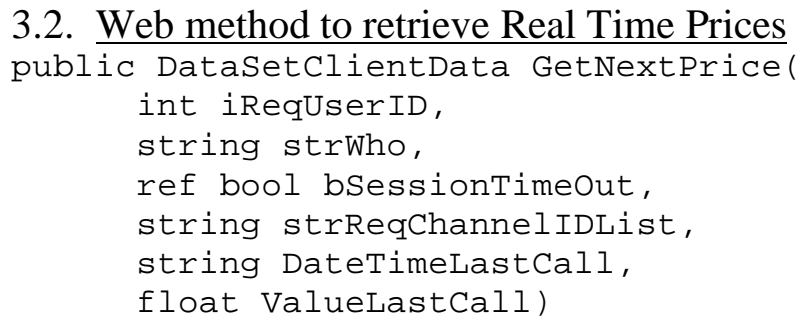

To call this web method, the client application must pass the following parameters:

- $\quad$ iReqUserID: set it to the userID parameter returned by the Web method Sessions_Login 
- strWho: set it to the strWho parameter returned by the Web method Sessions_Login

- strReqChannelIDList: Identifier of the price channel to retrieve (you will get this identifier from Infotility)

- DateTimelastcall: The timestamp of the price that was returned by this web method the last time it was called. It corresponds to the Date_Time field of the DataSetClientData.xsd dataset returned by the server. The format of this parameter is a string: "mm/dd/yyyy hh:mm:ss"

- ValueLastcall: The value of the price that was returned by the web service method the last time this web method was called.

This Web method returns a parameter (boolean): bsessionTimeout. This will be always false because the session duration will not be limited for the users of this project.

The Web method returns the price data in a dataset based on the RTPricing XML schema described below.

\subsection{Other web methods}

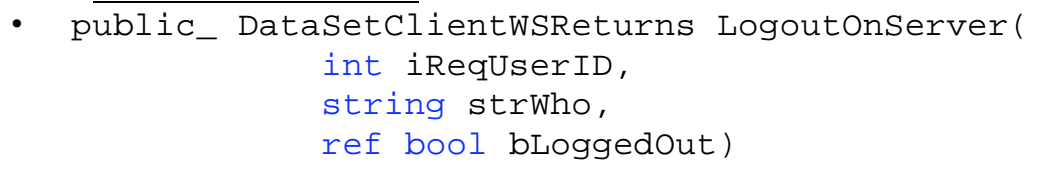

This method is used to logout. It is not required to use it because when the user logs in, the existing sessions are automatically cleared.

- DateTime GetSystemclock ()

This method the current UTC time on the server

\section{RT Pricing XML Schema}

The RTPricing XML schema is defined by the following schema (DataSetClientData.xsd):

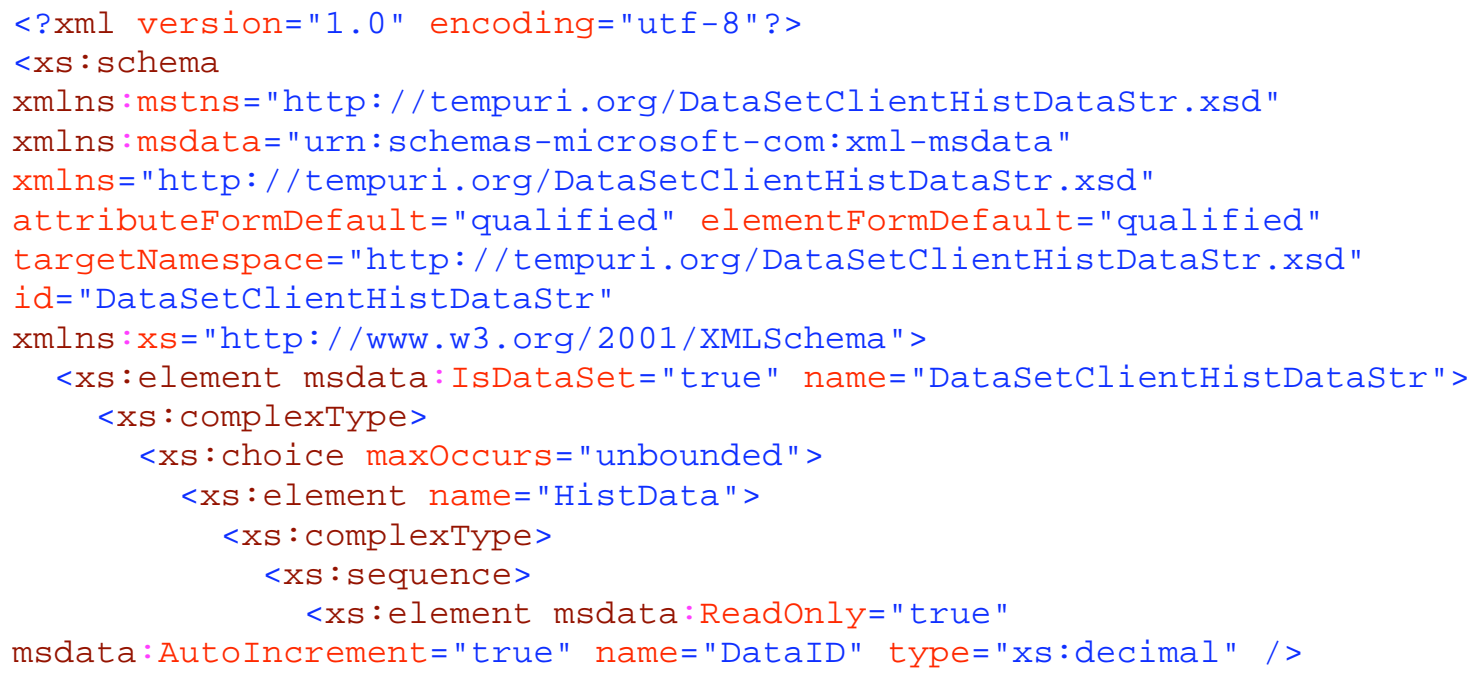




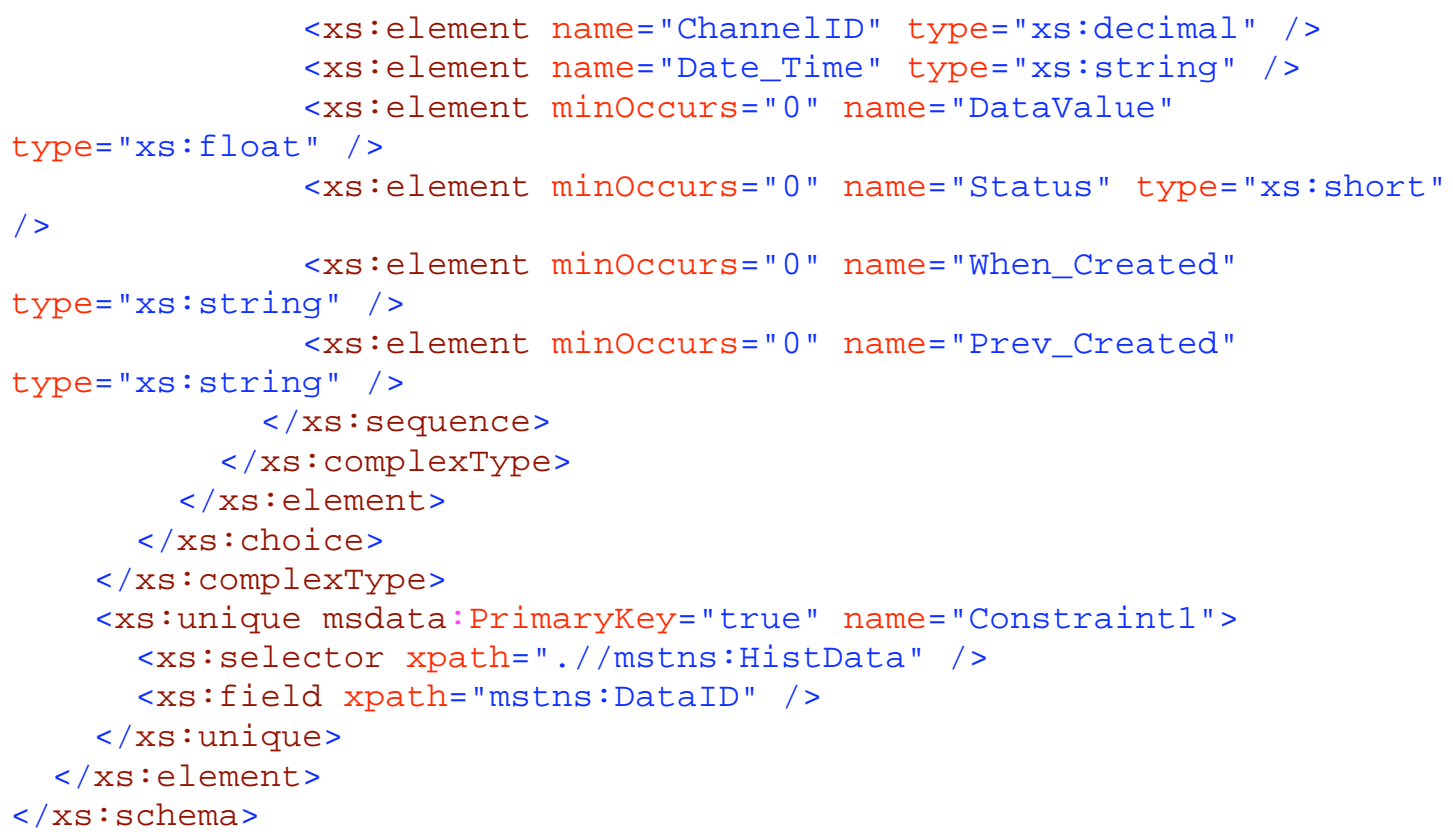

There are 6 elements:

- ChannelID: the ChannelID of the price

- Date_Time: it is the timestamp of the price. The format of this element is string: "mm/dd/yyyy hh:mm:ss"

- DataValue: it is a float number that contains the price value

- Status: It is a number that describes the DataValue. It is set to 1 for the prices of this project.

- When_Created: it is the date and time when this price value was received on the Infotility servers. The format of this element is string: "mm/dd/yyyy hh:mm:ss"

- Prev_Created: Not used for these prices

Bandwidth Requirements: Depending on the frequency of the RTP price delivery, the bandwidth requirements may vary; however, the data transfer rates are expected to remain low.

\section{Server URL}

The URL of the server wsdl is:

http://webservice01.infotility.com/lblwebsrv072803/servicelbl.asmx?wsdl

Note: Infotility is currently implementing https on this server. Https will be activated before 9/1/03. You will get an Email when it is ready. The new URL will be: https://webservice01.infotility.com/lblwebsrv072803/servicelbl.asmx?wsdl 



\section{Appendix IV. Participants List}

\section{Albertsons \\ Glenn Barrett \\ Scott Moore \\ Mike Water \\ Ed Lepacek \\ Paul Sheppard \\ Tom Pappas \\ John Kuhl \\ Jagdish Dudhat \\ Dean Cromwell \\ Cory Timms}

\section{Bank of America \\ Don Rudy \\ John Schinter \\ Steven Press \\ William Young \\ Kent Anderson \\ Dirk Mahling \\ Bob Landry \\ Glenn Miller}

GSA Oakland

Edgar Gray

John Goins

Bud Chisam

Stephen May

Mark Levi

\section{Roche}

Jerry Meek

Jeff Stamp

Ken Mason
Senior Manager / DSM, Albertsons

Albertsons

Local Store Manager, Albertsons

Senior Energy Coordinator, Albertsons

eLutions / Engage

eLutions / Engage

eLutions / Engage

eLutions / Engage

Aztec / Albertsons

West Valley Electric (sub of Aztec/Albertsons)

Senior Project Manager, Jones Lang LaSalle

Jones Lang LaSalle

Jones Lang LaSalle

Assistant Chief Engineer, Jones Lang LaSalle

Senior Facility Manager, Jones Lang LaSalle

Chief Technology Officer, Webgen Systems

Senior Project Manager, Webgen Systems

Account Manager, Webgen Systems

Property Manager, U.S. General Services Administration

Property Manager, U.S. General Services Administration

U.S. General Services Administration

U.S. General Services Administration

Building Management Specialist, U.S. General Services

Administration

Roche Palo Alto

Facility Manager, Roche Palo Alto

Yamas Controls, Inc / Tridium

\section{University of California, Santa Barbara}

Jim Dewey

Energy Manager, University of California, Santa Barbara

Dale Fong

Chief Technology Officer, Itron (Silicon Energy)

\section{United States Postal Services}

Ray Levinson

John Samuelson

Bill Golove
Pacific Area Environmental Compliance Manager, United States Postal Services

Building Manager, United States Postal Services

Staff Research Associate, Lawrence Berkeley National Laboratory 
Bruce Dickinson Business Development Manager, CMS Viron Energy Services / Chevron Energy Services

Infotility

Joseph Desmond

Nicolas Kardas
President and CEO, Infotility, Inc.

Sr. Director of Product Development, Infotility, Inc. 


\section{Appendix V. Site Questionnaires (Test 1, Test 2)}

\section{Appendix V-1. Site Questionnaires - Albertsons}

\section{Questions for after test 1}

Site: Albertson's store 7049

Questioner: David Watson

Questionee: Jagdish Dudhat (Engage Networks)

Date: $11 / 13 / 03$

All of these questions should be asked to on-site staff.

Questions with a SA - should also be asked of the system administrator or vendor

\section{Awareness - (SA)}

a. Were you aware that the price changed during the test on Wednesday? (SA) No

b. How did you know?

Did you have any automated alert function or other notification built into your system?

There is no automatic paging/notification system built in. He said that auto notification could be added as a feature, if desired.

i. How else did you find out?

1. observing a computer

2. observing the HVAC in zones

3. occupant feedback

4. other?

\section{Physical Response - (SA)}

Did the response strategy work as planned? Not applicable because he was not aware as described above.

i. If so, how do you know?

ii. If not, any sense why not?

\section{Implications of Test-}

a. Were there any operational issues as a results of the test the compromised the building services? Not applicable.

b. Was there any occupant feedback from modified operations?

i. Were tenants of customers aware?

ii. Any complaints or comfort issues?

\section{Any other comments?}




\section{Questions for after test 2}

Site: Albertson's store 7049

Questioner: David Watson

Questionee: Jagdish Dudhat (Engage Networks)

Date: $11 / 21 / 03$

All of these questions should be asked to on-site staff.

Questions with a SA - should also be asked of the system administrator or vendor

1. Awareness - (SA)

a. Were you aware that the price changed during the test on Wednesday? (SA) No

b. How did you know?

i. Did you have any automated alert function or other notification built into your system? There is no automatic paging/notification system built in. He said that auto notification could be added as a feature, if desired.

ii. How else did you find out?

1. observing a computer

2. observing the HVAC in zones

3. occupant feedback

4. other?

\section{Physical Response - (SA)}

Did the response strategy work as planned? Not applicable because he was not aware as described above.

iii. If so, how do you know?

iv. If not, any sense why not?

\section{Implications of Test-}

c. Were there any operational issues as a results of the test the compromised the building services? Not applicable.

d. Was there any occupant feedback from modified operations?

i. Were tenants of customers aware?

ii. Any complaints or comfort issues?

\section{Any other comments?}




\section{Appendix V-2. Site Questionnaires - Bank of America}

\section{Questions for After Test 1}

Questioner: David Watson

Jon Kream (WebGen)

Date: $11 / 18 / 2003$

All of these questions should be asked to on-site staff.

Questions with a SA - should also be asked of the system administrator or vendor

1. Awareness - (SA)

a. Were you aware that the price changed during the test on Wednesday? (SA) Yes.

b. How did you know?

i. Did you have any automated alert function or other notification built into your system? Yes.

ii. How else did you find out?

1. observing a computer

2. observing the HVAC in zones

3.

4. occupant feedback

5. other?

\section{Physical Response - (SA)}

a. Did the response strategy work as planned? No.

$i$. If so, how do you know?

ii. If not, any sense why not?

Buildings $A$ and $C$ responded but $B$ did not respond. Initially they thought that their signal did not go to Trane. However, after examining Trane trends (provided by $L B N L$ ), they realized that the signal went to Trane but Trane did not respond. They ran several tests on the $17^{\text {th }}$ and $18^{\text {th }}$ but were not able to pinpoint a problem. They think that it was an intermittent problem.

\section{Implications of Test}

a. Were there any operational issues as a results of the test the compromised the building services? No.

b. Was there any occupant feedback from modified operations?

i. Were tenants of customers aware? No.

ii. Any complaints or comfort issues? No.

\section{Any other comments?}

The response is such that the three buildings $A, B$, and $C$ work together to minimize the burden on each building. (This was not the plan LBNL, WebGen and BofA came up with-LBNL was expecting response only in $B$. 


\section{Questions for after test 2}

Questioner: David Watson

Questionee: Dirk Mahling (WebGen)

Date: $12 / 04 / 2003$

All of these questions should be asked to on-site staff.

Questions with a SA - should also be asked of the system administrator or vendor

\section{Awareness - (SA)}

a. Were you aware that the price changed during the test on Wednesday? (SA) Yes

b. How did you know? Manual observation. Change of price was observed through visual observation of a browser.

i. How else did you find out?

1. observing a computer

2. observing the HVAC in zones

3. occupant feedback

4. other?

\section{Physical Response - (SA)}

a. Did the response strategy work as planned? The shed strategy was to rotate reduction in duct static pressure setpoint and fan systems. This worked as planned on this test.

i. If so, how do you know?

ii. If not, any sense why not?

\section{Implications of Test}

a. Were there any operational issues as a results of the test the compromised the building services?

b. Was there any occupant feedback from modified operations?

i. Were tenants of customers aware? No.

ii. Any complaints or comfort issues? No.

\section{Any other comments?}

Tasks completed to implement Auto DR:

1) Write Web Services client. Client was written using Microsoft C++ Visual studio.

2) The price from the client was linked to the existing price input to the neural network agent. Note: this price input is normally linked to ISO streaming prices which are FTP based.

- Together, these tasks took about 2-3 days.

- Scalability: Since the price input to the neural network is a standard feature at all sites, it is simple to link additional sites to the web services client based price.

- Suggestions for improvement: Create a variable price with resolution of \$0.01 or greater. 


\section{Appendix V-3. Site Questionnaires - GSA Oakland}

\section{Questions for after test 1}

Questioner: David Watson

Questionee: Stephen May

Date: $11 / 13 / 2003$

All of these questions should be asked to on-site staff.

Questions with a SA - should also be asked of the system administrator or vendor

1. Awareness - (SA)

a. Were you aware that the price changed during the test on Wednesday? (SA) Yes

b. How did you know?

i. Did you have any automated alert function or other notification built into your system? Yes. Upon any change of price, an email is sent to: S. May, M. Levi, Susan C, Bill Goodner.

ii. How else did you find out?

1. observing a computer

2. observing the HVAC in zones

3. occupant feedback

4. other?

\section{Physical Response - (SA)}

a. Did the response strategy work as planned? No

i. If so, how do you know?

ii. If not, any sense why not?

The last device in the system "upstream" of the EMCS failed. The device that failed was an Alerton VLC controller. It received a command to energize either one or two relays, but the command was never acted upon. The Johnson EMCS is supposed to read the relays and initiate the shed accordingly.

Stephen May alerted Bill Goodner within 15 minutes of the change of price signal and asked him to visually inspect the state of the interface relays. When he said that they were not operating as expected S. May tried forcing the software to control the relays remotely to no avail. They declared the test a failure and disabled the relays. Next, they started to conduct internal testing and evaluation of the system. They are now conducting stress tests of the system. Even though there have been no changes to the system, it appears to be functional at this time. They plan to continue testing and then put the system back in automatic mode by Monday 11/17/03 8:00 AM PDT in preparation for the next LBNL price signal test.

Note: it is admirable that the participants did not physically force the system into a shed mode. The prompt timing of their attention to the system would have made it impossible for us to distinguish between automatic and manual initiation of the shed. 


\section{Implications of Test}

a. Were there any operational issues as a results of the test the compromised the building services? Not applicable.

b. Was there any occupant feedback from modified operations?

i. Were tenants of customers aware?

ii. Any complaints or comfort issues?

\section{Any other comments?}

\section{Questions for after test 2}

Questioner: David Watson

Questionee: Stephen May

Date: $11 / 21 / 2003$

All of these questions should be asked to on-site staff.

Questions with a SA - should also be asked of the system administrator or vendor

1. Awareness - (SA)

a. Were you aware that the price changed during the test on Wednesday? (SA) Yes

b. How did you know?

i. Did you have any automated alert function or other notification built into your system? Yes. Upon any change of price, an email is sent to: S. May, M. Levi, Susan C, Bill Goodner.

ii. How else did you find out?

1. observing a computer

2. observing the HVAC in zones

3. occupant feedback

4. other?

\section{Physical Response - (SA)}

a. Did the response strategy work as planned? The system was verified to have worked correctly all the way up to the interface relays to the Johnson system.

i. If so, how do you know?

Steven May saw over their network that the relays had been commanded. In addition, the status of the relays were visually verified by Building Engineer, Bill Goodner. Verification of the actions that occurred in the Johnson system and the HVAC system were not verified by S. May or B. Goodner.

ii. If not, any sense why not?

\section{Implications of Test}

a. Were there any operational issues as a results of the test the compromised the building services? Steven May could not provide this information. 
b. Was there any occupant feedback from modified operations?

i. Were tenants of customers aware?

ii. Any complaints or comfort issues?

\section{Any other comments?}




\section{Appendix V-4. Site Questionnaires - Roche}

\section{Questions after the test}

Questioner: Mary Ann Piette

Questionee: Jerry Meek (Roche)

Date: Test 1: 11/13/2003, Test 2: 11/24/2003

All of these questions should be asked to on-site staff.

Questions with a SA - should also be asked of the system administrator or vendor

\section{Awareness - (SA)}

a. Were you aware that the price changed during the test on Wednesday?

(SA) Test 1 - Yes

Test 2 - No

b. How did you know?

i. Did you have any automated alert function or other notification built into your system?

Test 1 - Yes. There is an alarm pt and we watched it.

Test 2 - Yes. Alarm on - but no one watched it.

ii. How else did you find out?

1. observing a computer

Test 1 -Yes. There is an alarm pt and we watched it.

2. observing the HVAC in zones

3. occupant feedback

4. other?

\section{Physical Response - (SA)}

a. Did the response strategy work as planned?

i. If so, how do you know?

Test 1 - Yes. There is an alarm pt and we watched it

Test 2 - Yes, but did not know until email congrats came on Friday, Nov 20.

ii. If not, any sense why not?

\section{Implications of Test-}

a. Were there any operational issues as a results of the test the compromised the building services? NO both tests

b. Was there any occupant feedback from modified operations?

i. Were tenants of customers aware?

ii. Any complaints or comfort issues?

NO both tests

\section{Any other comments?}

Jeff agrees that this could be an automated response to a utility tariff. $\mathrm{HE}$ WANTS TO SEE DRAFT PRESS RELEASE TO CLEAR IT. 


\section{Appendix V-5. Site Questionnaires - UCSB}

\section{Questions for after test 2}

Questioner: David Watson

Questionee: Dale Fong (Itron)

Date: $12 / 02 / 2003$

All of these questions should be asked to on-site staff.

Questions with a SA - should also be asked of the system administrator or vendor

\section{Awareness - (SA)}

a. Were you aware that the price changed during the test on Wednesday? (SA) Yes

b. How did you know? Manual observation. A real-time price "window" was left open on Dales' computer screen during the 2 week test period.

Did you have any automated alert function or other notification built into your system? There is no automatic paging/notification system built in. He said that auto notification could be added as a feature, if desired.

i. How else did you find out?

1. observing a computer

2. observing the HVAC in zones

3. occupant feedback

4. other?

\section{Physical Response - (SA)}

a. Did the response strategy work as planned? Although he did not have direct observation, Dale's understanding was that the shed strategies worked as planned..

i. If so, how do you know?

ii. If not, any sense why not?

\section{Implications of Test-}

a. Were there any operational issues as a results of the test the compromised the building services? No direct observation Not applicable.

b. Was there any occupant feedback from modified operations?

i. Were tenants of customers aware?

ii. Any complaints or comfort issues?

\section{Any other comments?}

Tasks completed to implement Auto DR:

1) Write Web Services client. Client was written using Borland DELPHI. Task took about 2 days.

2) Itron Universal Calculation Engine. This is where the control logic is implemented. Task took about 3 hours. 
3) On site work to the Johnson control system was performed by Jim Dewey. This work is described in the interview notes with Jim Dewey.

Scalablity: Dale said that for a large scale project (i.e. 10,000 + sites) that The Web Services server should "push" the pricing. Otherwise, as in this project, having the sites "pull" the price info is best. The Itron client (hosted locally at UCSB) pulled the Infotility server once per minute.

From the server side, many more sites could be brought on-line very quickly. From the control system side, several days per site would be necessary to add additional buildings or campuses.

Suggestions for improvement: Web Services server should be more stable custom clients are written. Web Services server could be more platform independent. It used Microsoft constructs in the server which made it more difficult to write clients using other development tools. Dale used Borland DELPHI. He spent several extra hours to overcome the Microsoft specific aspects of the server. He said that it would be even more difficult with a Linux or Java based development tool. 


\section{Appendix VI. Site Data Collection Points List}

\section{Appendix VI-1. Albertsons - Point List}

Table VI-1. Albertsons - eLutions Point List

\begin{tabular}{|c|c|c|c|}
\hline Category & Point Name & Unit & Interval \\
\hline Current Weather Data & NOAA-CA - Oakland CA & Deg-F & 60-minute \\
\hline Main Electric Meter & Main Electric Meter & $\mathrm{kW}$ & 15-minute \\
\hline \multirow[t]{2}{*}{ Start/Stop Loads } & Case Lights Start/Stop R-1 & Start/Stop & 15-minute \\
\hline & Case Lights Start/Stop R-2 & Start/Stop & 15-minute \\
\hline \multirow[t]{4}{*}{ Sub Metered Loads } & Anti-Sweat Door Heaters & $\mathrm{kW}$ & 15-minute \\
\hline & Case Lights 1 & $\mathrm{~kW}$ & 15-minute \\
\hline & Case Lights 2 & $\mathrm{~kW}$ & 15-minute \\
\hline & $\begin{array}{l}\text { Sales Lighting \& Misc. } \\
\text { (Overhead Lights) }\end{array}$ & $\mathrm{kW}$ & 15-minute \\
\hline XML Device & XML LBNL Device & $\$ / \mathrm{kWh}$ & 15-minute \\
\hline
\end{tabular}

\section{Appendix VI-2. Bank of America - Point List}

Table VI-2. BofA - WebGen Point List

\begin{tabular}{|c|l|c|c|}
\hline Fans & \multicolumn{1}{|c|}{ Point Name } & Unit & Interval \\
\hline \hline \multirow{5}{*}{ SF-1,2 } & BMS Static Pressure Setpoint & IWC & 15 -minute \\
\cline { 2 - 4 } & BMS Supply Air Setpoint & Deg-F & 15 -minute \\
\cline { 2 - 4 } & Building Static Pressure & IWC & 15 -minute \\
\cline { 2 - 4 } & Economizer Position & $\%$ & 15 -minute \\
\cline { 2 - 4 } & Flow SCHW & GPM & 15 -minute \\
\cline { 2 - 4 } & Return Capacity in CFM & CFM & 15 -minute \\
\cline { 2 - 4 } & Return Fan Status & On/Off & 15 -minute \\
\cline { 2 - 4 } & Space Temp & Deg-F & 15 -minute \\
\cline { 2 - 4 } & Speed Reset Override & $\%$ & 15 -minute \\
\cline { 2 - 4 } & Speed SF VFD & $\%$ & 15 -minute \\
\cline { 2 - 4 } & Static Pressure & IWC & 15 -minute \\
\cline { 2 - 4 } & Supply Air Temp Override & Deg-F & 15 -minute \\
\cline { 2 - 4 } & Supply Air Temp Read & Deg-F & 15 -minute \\
\cline { 2 - 4 } & Supply Air Temp Reset & Deg-F & 15 -minute \\
\cline { 2 - 4 } & Supply Capacity in CFM & CFM & 15 -minute \\
\cline { 2 - 4 } & Supply Fan Status & On/Off & 15 -minute \\
\cline { 2 - 4 } & Temp Avg Zone 2 & Deg-F & 15 -minute \\
\cline { 2 - 4 } & Temp Avg Zone 3 & Deg-F & 15 -minute \\
\cline { 2 - 4 } & Temp Avg Zone 4 & Deg-F & 15 -minute \\
\cline { 2 - 4 } & Temp Avg Zone 5 & Deg-F & 15 -minute \\
\cline { 2 - 4 } & Temp Avg Zone 6 & Deg-F & 15 -minute \\
\cline { 2 - 4 } & Temp Avg Zone 7 & Deg-F & 15 -minute \\
\cline { 2 - 4 } & Temp Avg Zone 8 & Deg-F & 15 -minute \\
\cline { 2 - 4 } & Temp MA & Deg-F & 15 -minute \\
\cline { 2 - 4 } & Temp OA & Deg-F & 15 -minute \\
\cline { 2 - 4 } & Temp RA & Deg-F & 15 -minute \\
\hline
\end{tabular}




\begin{tabular}{|c|c|c|c|}
\hline & WebGen Economizer Position Control Enable & $\%$ & 15-minute \\
\hline & WebGen Economizer Position Control & $\%$ & 15-minute \\
\hline & WebGen Static Pressure Reset Enable & IWC & 15-minute \\
\hline & WebGen Static Pressure Reset & IWC & 15-minute \\
\hline \multirow[t]{13}{*}{ SF-3,4 } & Building Static Pressure & IWC & 15-minute \\
\hline & Economizer Position & $\%$ & 15-minute \\
\hline & Flow SCHW & GPM & 15-minute \\
\hline & Return Capacity in CFM & CFM & 15-minute \\
\hline & Return Fan Status & On/Off & 15-minute \\
\hline & Speed RF VFD & $\%$ & 15-minute \\
\hline & Speed SF VFD & $\%$ & 15-minute \\
\hline & Static Pressure & IWC & 15-minute \\
\hline & Supply Air Temp Read & Deg-F & 15-minute \\
\hline & Supply Capacity in CFM & CFM & 15-minute \\
\hline & Supply Fan Status & On/Off & 15-minute \\
\hline & Temp MA & Deg-F & 15-minute \\
\hline & Temp RA & Deg-F & 15-minute \\
\hline
\end{tabular}

Table VI-3. BofA - Trane System Point List

\begin{tabular}{|l|l|c|c|}
\hline Category & \multicolumn{1}{|c|}{ Point Name } & Unit & Interval \\
\hline \hline \multirow{4}{*}{$\begin{array}{l}\text { Cooling } \\
\text { Energy }\end{array}$} & Building A CHW Supply & Tons & 15 -minute \\
\cline { 2 - 4 } & Building B CHW Supply & Tons & 15 -minute \\
\cline { 2 - 4 } & Building C CHW Supply & Tons & 15 -minute \\
\cline { 2 - 4 } & Building D CHW Supply & Tons & 15 -minute \\
\cline { 2 - 4 } & Building D Chiller Total & Wons & 15 -minute \\
\hline \multirow{5}{*}{$\begin{array}{l}\text { WebGen } \\
\text { Control }\end{array}$} & $\begin{array}{l}\text { Bldg A - Duct Static Pressure } \\
\text { Setpoint Reset Control }\end{array}$ & WebGen/Trane & 15 -minute \\
\cline { 2 - 4 } & Bldg A - Temp Setpoint Reset Control & Locked/Unlocked & 15 -minute \\
\cline { 2 - 4 } & Bldg A - VFD Lock Control & WebGen/Trane & 15 -minute \\
\cline { 2 - 4 } & $\begin{array}{l}\text { Bldg B - Duct Static Pressure } \\
\text { Setpoint Reset Control }\end{array}$ & WebGen/Trane & 15 -minute \\
\cline { 2 - 4 } & Bldg B - Temp Setpoint Reset Control & Locked/Unlocked & 15 -minute \\
\cline { 2 - 4 } & Bldg B - VFD Lock Control & WebGen/Trane & 15-minute \\
\cline { 2 - 4 } & $\begin{array}{l}\text { Bldg C - Duct Static Pressure } \\
\text { Setpoint Reset Control }\end{array}$ & WebGen/Trane & 15 -minute \\
\cline { 2 - 4 } & Bldg C - Temp Setpoint Reset Control & Locked/Unlocked & 15 -minute \\
\cline { 2 - 4 } & Bldg C - VFD Lock Control & & \\
\hline
\end{tabular}

Table VI-4. B of A - Submeter Point List

\begin{tabular}{|l|l|c|c|}
\hline File Name & \multicolumn{1}{|c|}{ Data Category } & Unit & Interval \\
\hline \hline \multirow{5}{*}{ EMCC } & MCC Phase A amps & Amps & 1-minute \\
\cline { 2 - 4 } & MCC Phase B amps & Amps & 1-minute \\
\cline { 2 - 4 } & MCC Phase C amps & Amps & 1-minute \\
\cline { 2 - 4 } & MCC Average amps & Amps & 1-minute \\
\cline { 2 - 4 } & MCC kva & $\mathrm{kWh}$ & 1-minute \\
\cline { 2 - 4 } & MCC Total kW & $\mathrm{kW}$ & 1-minute \\
\cline { 2 - 4 } & MCC Phase A kW & $\mathrm{kW}$ & 1-minute \\
\cline { 2 - 4 } & MCC Phase B kW & $\mathrm{kW}$ & 1-minute \\
\cline { 2 - 4 } & MCC Phase C kW & $\mathrm{kW}$ & 1-minute \\
\hline
\end{tabular}




\begin{tabular}{|l|l|c|c|}
\hline \multirow{5}{*}{} & MCC Average kW & $\mathrm{kW}$ & 1-minute \\
\cline { 2 - 4 } & MCC kWh & $\mathrm{kWh}$ & 1-minute \\
\cline { 2 - 4 } & MCC kW Max reached & $\mathrm{kW}$ & 1-minute \\
\cline { 2 - 4 } & MCC kW Min reached & $\mathrm{kW}$ & 1-minute \\
\cline { 2 - 4 } & MCC Power Factor & $\mathrm{PF}$ & 1-minute \\
\cline { 2 - 4 } & MCC Phase A power factor & $\mathrm{PF}$ & 1-minute \\
\cline { 2 - 4 } & MCC Phase B power factor & $\mathrm{PF}$ & 1-minute \\
\cline { 2 - 4 } & MCC Phase C power factor & $\mathrm{PF}$ & 1-minute \\
\cline { 2 - 4 } & MCC Phase A to B Phase volts & VAC & 1-minute \\
\cline { 2 - 4 } & MCC Phase A to C Phase volts & VAC & 1-minute \\
\cline { 2 - 4 } & MCC Phase C to B Phase volts & VAC & 1-minute \\
\cline { 2 - 4 } & MCC Phase line to line volts & VAC & 1-minute \\
\hline Amps EMCC & Exhaust Fan 3 VFD\&Motor Amps & Amps & 1-minute \\
\cline { 2 - 4 } & Exhaust Fan 4 VFD\&Motor Amps & Amps & 1-minute \\
\cline { 2 - 4 } & Supply Fan 3 VFD\&Motor Amps & Amps & 1-minute \\
\cline { 2 - 4 } & Supply Fan 4 VFD\&Motor Amps & Amps & 1-minute \\
\cline { 2 - 4 } & Exhaust Fan 1 VFD\&Motor Amps & Amps & 1-minute \\
\cline { 2 - 4 } & Exhaust Fan 2 VFD\&Motor Amps & Amps & 1-minute \\
\cline { 2 - 4 } & Supply Fan 1 VFD\&Motor Amps & Amps & 1-minute \\
\cline { 2 - 4 } & Supply Fan 2 VFD\&Motor Amps & Amps & 1-minute \\
\hline MCC Amps & MCC Amps Value & Amps & 1-minute \\
\hline \hline B2PWR & Building B - Power & 1-minute \\
\hline
\end{tabular}

Appendix VI-3. GSA Oakland - Point List

Table VI-5. GSA Oakland - InterAct Point List

\begin{tabular}{|c|l|c|c|}
\hline Meter \# & \multicolumn{1}{|c|}{ Description } & Unit & Interval \\
\hline \hline Meter-1 & North tower lighting & $\mathrm{kWh}$ & 15 -minute \\
\hline Meter-2 & South tower basement lighting & $\mathrm{kWh}$ & 15 -minute \\
\hline Meter-3 & North tower mechanical room, cooling tower & $\mathrm{kWh}$ & 15 -minute \\
\hline Meter-4 & Central plant & $\mathrm{kWh}$ & 15 -minute \\
\hline Meter-5 & South tower mechanical room, auditorium & $\mathrm{kWh}$ & 15 -minute \\
\hline
\end{tabular}

Table VI-6. GSA Oakland - EMCS Point List

Data points used for or relevant to the saving analysis

\begin{tabular}{|l|l|l|l|}
\hline \multicolumn{1}{|c|}{ Location/Equipment } & \multicolumn{1}{|c|}{ Point Description } & \multicolumn{1}{c|}{ Unit } & \multicolumn{1}{c|}{ Interval } \\
\hline \hline Zone Temperature & & & \\
\hline North Tower & & & \\
\hline 17th floor (4 zones) & zone temperature (monitored) & Deg-F & $5 \mathrm{~min}$ \\
\hline 16th floor (5 zones) & zone temperature (monitored) & Deg-F & $5 \mathrm{~min}$ \\
\cline { 2 - 4 } & zone temperature setpoint & Deg-F & $5 \mathrm{~min}$ \\
\cline { 2 - 4 } & VAV box damper position & $\%$ & $5 \mathrm{~min}$ \\
\cline { 2 - 4 } & airflow (monitored) & CFM & $5 \mathrm{~min}$ \\
\cline { 2 - 4 } & airflow setpoint & CFM & $5 \mathrm{~min}$ \\
\hline 12th floor (4 zones) & zone temperature (monitored) & Deg-F & $5 \mathrm{~min}$ \\
\hline 6th floor (4 zones) & zone temperature (monitored) & Deg-F & $5 \mathrm{~min}$ \\
\hline 4th floor (4 zones) & zone temperature (monitored) & Deg-F & $5 \mathrm{~min}$ \\
\cline { 2 - 5 } & zone temperature setpoint & Deg-F & $5 \mathrm{~min}$ \\
\hline
\end{tabular}




\begin{tabular}{|c|c|c|c|}
\hline & VAV box damper position & $\%$ & $5 \mathrm{~min}$ \\
\hline & airflow (monitored) & CFM & $5 \mathrm{~min}$ \\
\hline & airflow setpoint & CFM & $5 \mathrm{~min}$ \\
\hline 2nd floor (4 zones) & zone temperature (monitored) & Deg-F & $5 \mathrm{~min}$ \\
\hline \multicolumn{4}{|l|}{ South Tower } \\
\hline \multirow[t]{5}{*}{ 16th floor (5 zones) } & zone temperature (monitored) & Deg-F & $5 \mathrm{~min}$ \\
\hline & zone temperature setpoint & Deg-F & $5 \mathrm{~min}$ \\
\hline & VAV box damper position & $\%$ & $5 \mathrm{~min}$ \\
\hline & airflow (monitored) & CFM & $5 \mathrm{~min}$ \\
\hline & airflow setpoint & CFM & $5 \mathrm{~min}$ \\
\hline \multirow[t]{5}{*}{ 4th floor (4 zones) } & zone temperature (monitored) & Deg-F & $5 \mathrm{~min}$ \\
\hline & zone temperature setpoint & Deg-F & $5 \mathrm{~min}$ \\
\hline & VAV box damper position & $\%$ & $5 \mathrm{~min}$ \\
\hline & airflow (monitored) & CFM & $5 \mathrm{~min}$ \\
\hline & airflow setpoint & CFM & $5 \mathrm{~min}$ \\
\hline 2nd floor (4 zones) & zone temperature (monitored) & Deg-F & $5 \mathrm{~min}$ \\
\hline \multicolumn{4}{|l|}{ Cooling Plant } \\
\hline \multirow{8}{*}{$\begin{array}{l}\text { Chiller CH-1 5 } \\
\text { (5 units) }\end{array}$} & chiller power & $\mathrm{kW}$ & $5 \mathrm{~min}$ \\
\hline & chiller status & on/off & $5 \mathrm{~min}$ \\
\hline & CHWS temperature (monitored) & Deg-F & $5 \sim 15 \mathrm{~min}$ \\
\hline & CHWS temperature setpoint & Deg-F & $5 \sim 15 \mathrm{~min}$ \\
\hline & CHWR temperature & Deg-F & $5 \sim 15 \mathrm{~min}$ \\
\hline & CHW flow & GPM & $5 \sim 15 \mathrm{~min}$ \\
\hline & CHW cooling energy & Btu/h & $5 \sim 15 \mathrm{~min}$ \\
\hline & Chiller load & & \\
\hline \multirow{3}{*}{$\begin{array}{l}\text { CHW Pump CHP-1 5 } \\
\text { (5 units) }\end{array}$} & Primary pump status & on/off & $5 \mathrm{~min}$ \\
\hline & Secondary pump status & on/off & $5 \mathrm{~min}$ \\
\hline & Secondary pump VFD speed & $\%$ & $5 \mathrm{~min}$ \\
\hline $\begin{array}{l}\text { CW Pump CP-1 5 } \\
\text { (5 units) }\end{array}$ & CW pump status & on/off & $5 \mathrm{~min}$ \\
\hline \multirow[t]{2}{*}{ Cooling tower (4 units) } & fan status & on/off & $5 \mathrm{~min}$ \\
\hline & Fan VFD (CT-1D only) & $\%$ & $5 \mathrm{~min}$ \\
\hline \multicolumn{4}{|l|}{ Fan System } \\
\hline \multicolumn{4}{|l|}{ North tower } \\
\hline \multirow{9}{*}{$\begin{array}{l}5 \text { th floor } \\
(2 \text { cold deck fans, } \\
1 \text { hot deck fans, } \\
2 \text { return fans })\end{array}$} & Fan status & on/off & $5 \mathrm{~min}$ \\
\hline & Fan VFD & $\%$ & $5 \mathrm{~min}$ \\
\hline & Cooling valve position & $\%$ & $5 \mathrm{~min}$ \\
\hline & Heating valve position & $\%$ & $5 \mathrm{~min}$ \\
\hline & Differential pressure & IWC & $5 \mathrm{~min}$ \\
\hline & Supply air temperature & Deg-F & $5 \mathrm{~min}$ \\
\hline & Mixed air temperature & Deg-F & $5 \mathrm{~min}$ \\
\hline & Return air temperature & Deg-F & $5 \mathrm{~min}$ \\
\hline & Static pressure & Deg-F & $5 \mathrm{~min}$ \\
\hline \multirow{5}{*}{$\begin{array}{l}\text { Penthouse } \\
(2 \text { cold deck fans, } \\
2 \text { hot deck fans, } \\
2 \text { return fans) }\end{array}$} & Fan status & on/off & $5 \mathrm{~min}$ \\
\hline & Fan VFD & $\%$ & $5 \mathrm{~min}$ \\
\hline & Cooling valve position & $\%$ & $5 \mathrm{~min}$ \\
\hline & Heating valve position & $\%$ & $5 \mathrm{~min}$ \\
\hline & Differential pressure & IWC & $5 \mathrm{~min}$ \\
\hline
\end{tabular}




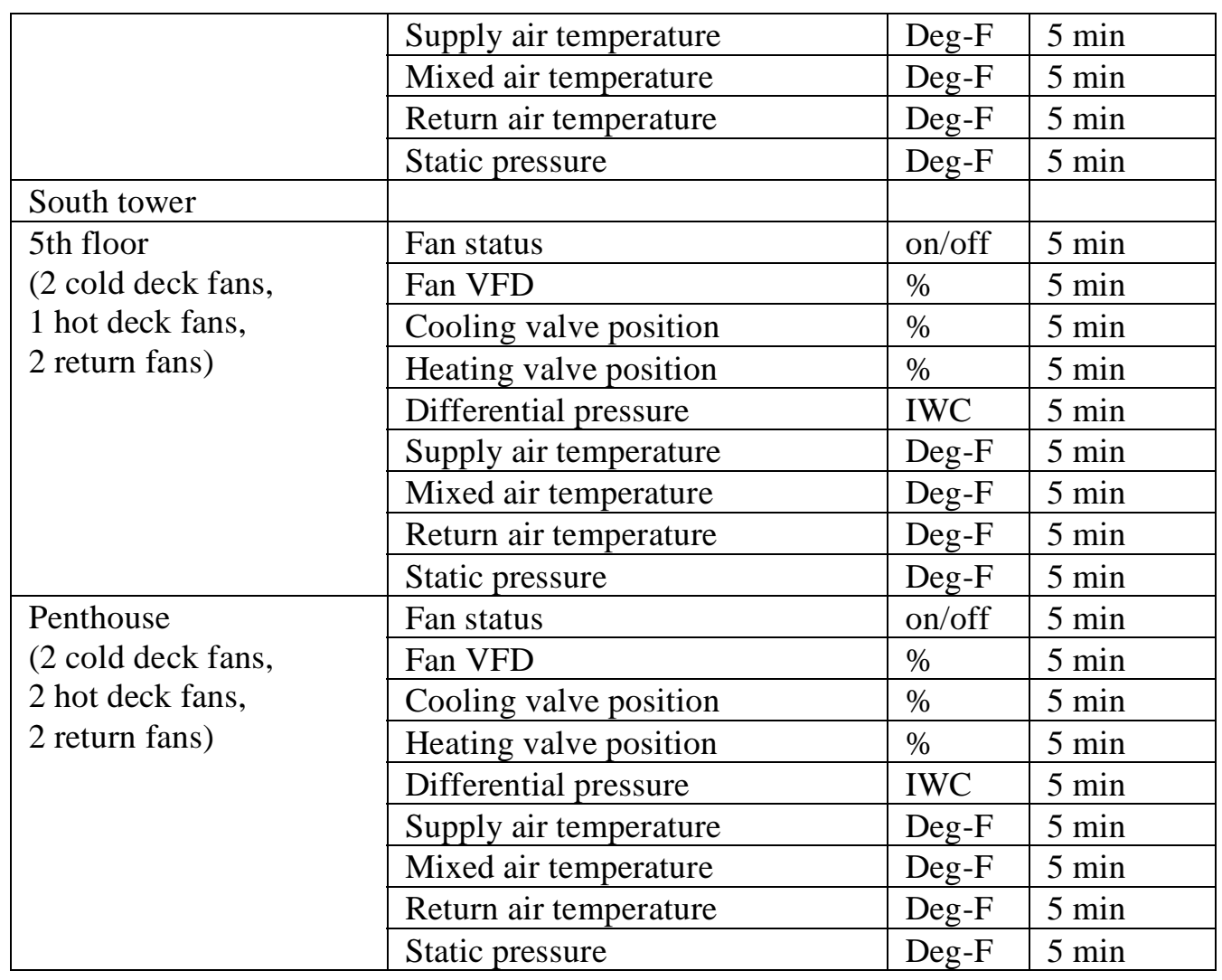

Table VI-7. GSA Oakland - Submeter Point List

\begin{tabular}{|l|l|c|}
\hline \multicolumn{1}{|c|}{ Equipment } & \multicolumn{1}{|c|}{ Description } & Interval \\
\hline CHP-NB-6 & Secondary chilled water pump with VFD & 1-minute \\
\hline CHP-NB-8 & Secondary chilled water pump with VFD & 1-minute \\
\hline SF-SP-1 & South tower penthouse cold deck fan & 1-minute \\
\hline SF-SP-2 & South tower penthouse cold deck fan & 1-minute \\
\hline SF-S5-1 & South tower 5th floor cold deck fan & 1-minute \\
\hline SF-S5-2 & South tower 5th floor cold deck fan & 1-minute \\
\hline
\end{tabular}

Trend data are 3 phases and average of kW, Amps, Power Factor and Volt.

\section{Appendix VI-4. Roche - Point List and Fan Spot Measurement Plan}

Table VI-8. Roche - PML PegaSys Point List

\begin{tabular}{|l|c|c|}
\hline \multicolumn{1}{|c|}{ Point Name } & Unit & Interval \\
\hline Building A2 - Whole Building Power & $\mathrm{kW}$ & 15 -minute \\
\hline Building SS - Whole Building Power & $\mathrm{kW}$ & 15 -minute \\
\hline Building FS - Whole Building Power & $\mathrm{kW}$ & 15 -minute \\
\hline
\end{tabular}


Table VI-9. Roche - Real-Time Energy Tracker Point List

\begin{tabular}{|c|c|c|c|}
\hline Category & Point Name & Unit & Interval \\
\hline \multirow[t]{7}{*}{ Logical Status } & A2 Fan-2 Status & Start/Stop & 5-minute \\
\hline & A2 Fan-4 Status & Start/Stop & 5-minute \\
\hline & A2 Fan-6 Status & Start/Stop & 5-minute \\
\hline & FS Supply Fan-1 Status & Start/Stop & 5-minute \\
\hline & FS Supply Fan-2 Status & Start/Stop & 5-minute \\
\hline & FS Return Fan-1 Status & Start/Stop & 5-minute \\
\hline & SS AH-04 Status & Start/Stop & 5-minute \\
\hline \multirow[t]{3}{*}{ Price Signal } & Equipment Status 10 Cents Plan & $\$ / \mathrm{kWh}$ & 5-minute \\
\hline & Equipment Status 30 Cents Plan & $\$ / \mathrm{kWh}$ & 5-minute \\
\hline & Equipment Status 75 Cents Plan & $\$ / \mathrm{kWh}$ & 5-minute \\
\hline \multirow[t]{6}{*}{ Temperature } & A2 SF-1,2 OSA Temperature & Deg-F & 5-minute \\
\hline & A2 SF-3,4 OSA Temperature & Deg-F & 5-minute \\
\hline & A2 SF-5,6 OSA Temperature & Deg-F & 5-minute \\
\hline & A2 SF-1,2 Zone Temperature & Deg-F & 5-minute \\
\hline & A2 SF-3,4 Zone Temperature & Deg-F & 5-minute \\
\hline & A2 SF-5,6 Zone Temperature & Deg-F & 5-minute \\
\hline \multirow[t]{3}{*}{$\mathrm{CO}_{2}$} & A2 SF-1,2 $\mathrm{CO}_{2}$ Concentration & $\mathrm{ppm}$ & 5-minute \\
\hline & $\mathrm{A} 2 \mathrm{SF}-3,4 \mathrm{CO}_{2}$ Concentration & ppm & 5-minute \\
\hline & $\mathrm{A} 2 \mathrm{SF}-5,6 \mathrm{CO}_{2}$ Concentration & ppm & 5-minute \\
\hline
\end{tabular}

\section{Roche - Fan M\&V Spot Measurement Plan (by Kris Kinnev)}

PML - Roche is going to generate a service call with PML to have the database and time stamp reviewed and brought into current. We need to follow up on that so we can make sure that any current data is captured before the rework. More important is to determine the exact offset for the data files. There was no schedule determined on when this would be done.

Building schedules:

SS - Site services - Start 7 a.m. to $6 \mathrm{pm}$

FS - Food Services - Starts 4:30 a.m. to 5 pm

A2 - Office building - Starts 6 a.m. to 6 pm

\section{SS Building}

SS AH4

Thursday 09/30/03 13:38 hours - measurement taken at MCC.

Supply Fans have power factor correction capacitors. Exhaust fans did not. 5 to $7.5 \mathrm{~kW}, 480$ to 490 volts, power factor (PF) 0.51, Around $30 \mathrm{HP}$ motor.

SS EF

482 to 491 volts, 1380 to 1480 watts - Measurement take at disconnect at fan. Around 15 HP motor. 


\section{FS Building}

Measurements taken on 10/15/03 at 11:00 a.m. - both fans running.

Supply Fans have power factor correction capacitors. Exhaust did not.

\section{FSSF01 Fan 4S-1}

Voltage $510 \mathrm{~V}, 12.3$ to $12.5 \mathrm{~kW}, \mathrm{PF}=.87$, no vfd control, Around $25 \mathrm{HP}$.

FSSF02 Fan 4S-2

Voltage 500 to $507 \mathrm{~V}, 12.8 \mathrm{~kW}, \mathrm{PF}=.78$, no vfd control, Around $25 \mathrm{HP}$.

Common exhaust Fan FE-1

Volts $=500,5.4 \mathrm{~kW}, \mathrm{PF}=0.58$

This fan is not interlocked with the two supply fans. Independently controlled.

FSSF01 fan in solo without FSSF02

$500 \mathrm{~V}, 8$ to $9.1 \mathrm{~kW}$, Exhaust was almost the same.

\section{A2 Building}

Taken on 10/15/03 from 1 to $2 \mathrm{pm}$. Each section was done within 10 to 15 minutes.

\section{Section 1 of three}

Identical fan sections installed over three wings.

Supply and return are interlocked. Section 3 only had one exhaust fan.

Supply fans had power factor correction and exhaust did not. No vfds for control.

\begin{tabular}{|l|c|c|c|}
\hline & $\mathrm{kW}$ & $\mathrm{PF}$ & Volts \\
\hline Fan 3S-3 (A2SF03) & $24.2-25.1 \mathrm{~kW}$ & 0.66 & $490-496 \mathrm{~V}$ \\
\hline Fan 3S-4 (A2SF04) & $27.8-28.95 \mathrm{~kW}$ & 0.67 & $490 \mathrm{~V}$ \\
\hline Fan 3E-3 (A2RA03) & $6.5 \mathrm{~kW}$ & 0.78 & $490 \mathrm{~V}$ \\
\hline Fan 3E-4 (A2RA04) & $4.88-4.92 \mathrm{~kW}$ & 0.55 & $493-500 \mathrm{~V}$ \\
\hline Fan 3S-3 (A2SF03) ran in solo & $29.88 \mathrm{~kW}$ & 0.83 & $495 \mathrm{~V}$ \\
\hline Fan 3E-3 (A2SF04) ran in solo & $7 \mathrm{~kW}$ & 0.84 & $495 \mathrm{~V}$ \\
\hline
\end{tabular}

\section{Section 2 of three}

Identical fan sections installed over three wings. Supply and return are interlocked. Section 3 only had one exhaust fan. Supply fans had power factor correction and exhaust did not. No VFDs for control.

\begin{tabular}{|l|c|c|c|}
\hline & $\mathrm{kW}$ & $\mathrm{PF}$ & Volts \\
\hline Fan 3S-1 (A2SF01) & $18.4 \mathrm{~kW}$ & 0.77 & $495 \mathrm{~V}$ \\
\hline Fan 3S-2 (A2SF02) & $21.8 \mathrm{~kW}$ & 0.81 & $495 \mathrm{~V}$ \\
\hline Fan 3E-1 (A2RA01) & $4 \mathrm{~kW}$ & 0.50 & $491 \mathrm{~V}$ \\
\hline Fan 3E-2 (A2RA02) & $4.36 \mathrm{~kW}$ & 0.50 & $495 \mathrm{~V}$ \\
\hline Fan 3S-1 (A2SF01) ran in solo & $20.9 \mathrm{~kW}$ & 0.79 & $495 \mathrm{~V}$ \\
\hline Fan 3E-1 (A2RA01) ran in solo & $4.4 \mathrm{~kW}$ & 0.51 & $499 \mathrm{~V}$ \\
\hline
\end{tabular}


Section 3 of three

Identical fan sections installed over three wings. Supply and return at not interlocked. Supply fans had power factor correction and exhaust did not. No VFDs for control.

\begin{tabular}{|l|c|c|c|}
\hline & $\mathrm{kW}$ & PF & Volts \\
\hline Fan 3S-5 (A2SF05) & $21 \mathrm{~kW}$ & 0.79 & $494 \mathrm{~V}$ \\
\hline Fan 3S-6 (A2SF06) & $19.2 \mathrm{~kW}$ & 0.83 & $497 \mathrm{~V}$ \\
\hline Fan 3E-5 (A2RA05) & $5.2 \mathrm{~kW}$ & 0.56 & $497 \mathrm{~V}$ \\
\hline Fan 3S-5 (A2SF05) ran in solo & $20.6 \mathrm{~kW}$ & 0.79 & $494 \mathrm{~V}$ \\
\hline Fan 3E-5 (A2RA05) ran in solo & $5.1 \mathrm{~kW}$ & 0.56 & $497 \mathrm{~V}$ \\
\hline
\end{tabular}




\section{Appendix VI-5. UCSB - Shed Strategies and Point List}

Table VI-10. UCSB - Shed Strategies Summary

\begin{tabular}{|c|c|c|c|c|c|c|}
\hline \multirow[b]{3}{*}{ System } & \multirow[b]{3}{*}{ Supply to } & \multirow[b]{3}{*}{ Point } & \multirow[b]{3}{*}{ DRP description } & \multicolumn{3}{|c|}{ DRP reduction } \\
\hline & & & & Level-1 & Level-2 & Level-3 \\
\hline & & & & Command & Command & Command \\
\hline $525-2 S 1$ & Library II S/E & $\begin{array}{l}\text { SF-CMD } \\
\text { C-VLV-C } \\
\text { H-VLV-C } \\
\text { E-DPR-C }\end{array}$ & $\begin{array}{l}\text { Supply Fan VFD Command } \\
\text { Cooling Valve Position } \\
\text { Heating Valve Position } \\
\text { Economizer \% OSA }\end{array}$ & $70 \%$ & $100 \%$ & $\begin{array}{r}60 \% \\
0 \% \\
0 \% \\
100 \%\end{array}$ \\
\hline $525-2 S 2$ & Library II N/E & $\begin{array}{l}\text { SF-CMD } \\
\text { C-VLV-C } \\
\text { H-VLV-C } \\
\text { E-DPR-C }\end{array}$ & $\begin{array}{l}\text { Supply Fan VFD Command } \\
\text { Cooling Valve Position } \\
\text { Heating Valve Position } \\
\text { Economizer \% OSA }\end{array}$ & $70 \%$ & $60 \%$ & $\begin{array}{r}60 \% \\
0 \% \\
0 \% \\
100 \%\end{array}$ \\
\hline $525-2 S 3$ & Library II N/Center & $\begin{array}{l}\text { SF-CMD } \\
\text { C-VLV-C } \\
\text { H-VLV-C } \\
\text { E-DPR-C } \\
\end{array}$ & $\begin{array}{l}\text { Supply Fan VFD Command } \\
\text { Cooling Valve Position } \\
\text { Heating Valve Position } \\
\text { Economizer \% OSA } \\
\end{array}$ & $100 \%$ & $60 \%$ & $\begin{array}{r}60 \% \\
0 \% \\
0 \% \\
100 \% \\
\end{array}$ \\
\hline $525-2 S 4$ & Library II S/Center & $\begin{array}{l}\text { SF-CMD } \\
\text { C-VLV-C } \\
\text { H-VLV-C } \\
\text { E-DPR-C }\end{array}$ & $\begin{array}{l}\text { Supply Fan VFD Command } \\
\text { Cooling Valve Position } \\
\text { Heating Valve Position } \\
\text { Economizer \% OSA }\end{array}$ & $70 \%$ & $100 \%$ & $\begin{array}{r}60 \% \\
0 \% \\
0 \% \\
100 \%\end{array}$ \\
\hline $525-2 S 5$ & Library II W/LS & $\begin{array}{l}\text { SF-CMD } \\
\text { C-VLV-C } \\
\text { H-VLV-C } \\
\text { E-DPR-C } \\
\end{array}$ & $\begin{array}{l}\text { Supply Fan VFD Command } \\
\text { Cooling Valve Position } \\
\text { Heating Valve Position } \\
\text { Economizer \% OSA } \\
\end{array}$ & $70 \%$ & $\begin{array}{r}60 \% \\
100 \% \\
\end{array}$ & $\begin{array}{r}60 \% \\
0 \% \\
0 \% \\
100 \% \\
\end{array}$ \\
\hline 525-3S1 & Library III 4-7F West & $\begin{array}{l}\text { SF-CMD } \\
\text { C-VLV-C } \\
\text { H-VLV-C } \\
\text { E-DPR-C }\end{array}$ & $\begin{array}{l}\text { Supply Fan VFD Command } \\
\text { Cooling Valve Position } \\
\text { Heating Valve Position } \\
\text { Economizer \% OSA }\end{array}$ & & & $\begin{array}{r}0 \% \\
0 \% \\
100 \% \\
\end{array}$ \\
\hline $525-3 S 2$ & Library III 1-3F West & $\begin{array}{l}\text { SF-CMD } \\
\text { C-VLV-C } \\
\text { H-VLV-C } \\
\text { E-DPR-C }\end{array}$ & $\begin{array}{l}\text { Supply Fan VFD Command } \\
\text { Cooling Valve Position } \\
\text { Heating Valve Position } \\
\text { Economizer \% OSA }\end{array}$ & $\begin{array}{r}70 \% \\
100 \% \\
\end{array}$ & $\begin{array}{r}60 \% \\
100 \% \\
\end{array}$ & $\begin{array}{r}60 \% \\
0 \% \\
0 \% \\
100 \% \\
\end{array}$ \\
\hline $525-3 S 3$ & $8 \mathrm{~F}$ & $\begin{array}{l}\text { SF-CMD } \\
\text { C-VLV-C } \\
\text { H-VLV-C } \\
\text { E-DPR-C } \\
\end{array}$ & $\begin{array}{l}\text { Supply Fan VFD Command } \\
\text { Cooling Valve Position } \\
\text { Heating Valve Position } \\
\text { Economizer \% OSA } \\
\end{array}$ & & & \\
\hline 525-3S4 & Library III 4-7F East & $\begin{array}{l}\text { SF-CMD } \\
\text { C-VLV-C } \\
\text { H-VLV-C } \\
\text { E-DPR-C } \\
\end{array}$ & $\begin{array}{l}\text { Supply Fan VFD Command } \\
\text { Cooling Valve Position } \\
\text { Heating Valve Position } \\
\text { Economizer \% OSA } \\
\end{array}$ & & & $\begin{array}{r}0 \% \\
0 \% \\
100 \% \\
\end{array}$ \\
\hline 525-3S5 & Library III 1-3F East & $\begin{array}{l}\text { SF-CMD } \\
\text { C-VLV-C } \\
\text { H-VLV-C } \\
\text { E-DPR-C }\end{array}$ & $\begin{array}{l}\text { Supply Fan VFD Command } \\
\text { Cooling Valve Position } \\
\text { Heating Valve Position } \\
\text { Economizer \% OSA }\end{array}$ & & & \\
\hline $525-4 S 1$ & $\begin{array}{l}\text { Library IV West } \\
\text { (large open space) }\end{array}$ & \begin{tabular}{|l|} 
SP-SET \\
C-VLV-C \\
DPR-C \\
\end{tabular} & $\begin{array}{l}\text { Static Set (.6 IWC) 525-2S1C } \\
\text { Cooling Valve Position } \\
\text { Economizer \% OSA }\end{array}$ & & $\begin{array}{r}0.4 \\
100 \% \\
\end{array}$ & $\begin{array}{r}0.4 \\
0 \% \\
100 \% \\
\end{array}$ \\
\hline $525-4 S 2$ & $\begin{array}{l}\text { Library IV East } \\
\text { (large open space) }\end{array}$ & $\begin{array}{l}\text { SP-SET } \\
\text { C-VLV-C } \\
\text { DPR-C } \\
\end{array}$ & $\begin{array}{l}\text { Static Set (.6 IWC) 525-2S2C } \\
\text { Cooling Valve Position } \\
\text { Economizer \% OSA }\end{array}$ & & $\begin{array}{r}0.4 \\
100 \% \\
\end{array}$ & $\begin{array}{r}0.4 \\
0 \% \\
100 \% \\
\end{array}$ \\
\hline $525-4 S 3$ & Library IV W-peri & $\begin{array}{l}\text { C-VLV-C } \\
\text { H-VLV-C }\end{array}$ & $\begin{array}{l}\text { Cooling Valve Position } \\
\text { Heating Valve Position }\end{array}$ & & & $\begin{array}{l}0 \% \\
0 \%\end{array}$ \\
\hline $525-4 S 4$ & Library IV S-peri & $\begin{array}{l}\text { SF-CMD } \\
\mathrm{H}-\mathrm{VLV}-\mathrm{C} \\
\end{array}$ & $\begin{array}{l}\text { Cooling Valve Position } \\
\text { Heating Valve Position }\end{array}$ & & & $\begin{array}{l}0 \% \\
0 \% \\
\end{array}$ \\
\hline $525-4 S 5$ & Library IV E-peri & $\begin{array}{l}\text { SF-CMD } \\
\text { H-VLV-C }\end{array}$ & $\begin{array}{l}\text { Cooling Valve Position } \\
\text { Heating Valve Position }\end{array}$ & & & $\begin{array}{l}0 \% \\
0 \%\end{array}$ \\
\hline
\end{tabular}


Table VI-11. UCSB - EEM Suite Point Summary

\begin{tabular}{|c|c|c|c|c|c|c|c|c|c|c|c|c|c|c|c|c|c|c|c|c|}
\hline \multicolumn{21}{|l|}{ Fan Trend } \\
\hline \multicolumn{2}{|c|}{ Interval } & \multicolumn{16}{|c|}{ 15-minute } & \multicolumn{3}{|c|}{ 5-minute } \\
\hline \multicolumn{2}{|c|}{ Point Category } & 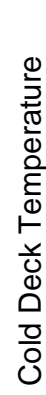 & 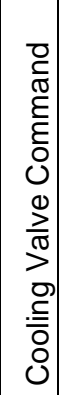 & 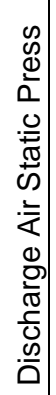 & 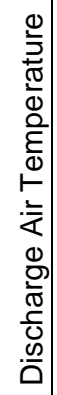 & 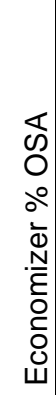 & 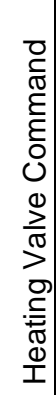 & 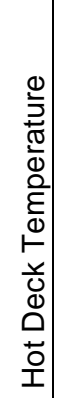 & 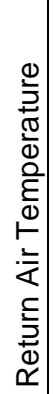 & 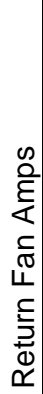 & 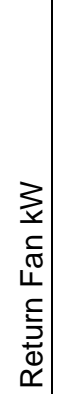 & 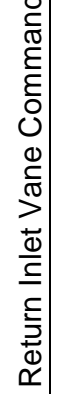 & 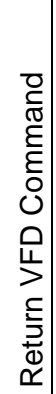 & $\begin{array}{l}0 \\
0 \\
\frac{0}{2} \\
\frac{1}{\sigma} \\
\leftarrow \\
\frac{7}{0} \\
0 \\
0\end{array}$ & 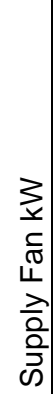 & 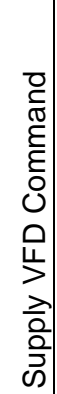 & 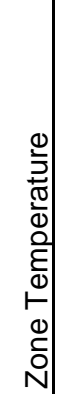 & 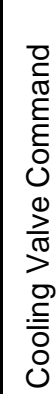 & 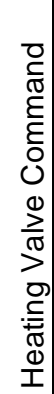 & 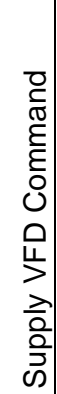 \\
\hline \multirow{5}{*}{ Library II } & S-1 Fan & $\overline{11}$ & 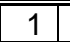 & & & 1 & 1 & 1 & $\overline{11}$ & 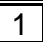 & & & & & $\overline{11}$ & 1 & & $\overline{11}$ & $\overline{11}$ & 1 \\
\hline & S-2 Fan & 1 & 1 & & & 1 & 1 & 1 & & 1 & & & & & 1 & 1 & 1 & 1 & 1 & 1 \\
\hline & S-3 Fan & 1 & 1 & & & 1 & 1 & 1 & & 1 & & & & & 1 & 1 & 1 & 1 & 1 & 1 \\
\hline & S-4 Fan & 1 & 1 & & & 1 & 1 & 1 & & 1 & & & & & 1 & 1 & 2 & & & 1 \\
\hline & S-5 Fan & 1 & 1 & & & 1 & 1 & 1 & & 1 & & & & & 1 & 1 & 2 & & & 1 \\
\hline \multirow{5}{*}{ Library III } & S-1 Fan & $\overline{1}$ & $\overline{1}$ & & & $\overline{11}$ & $\overline{1}$ & $\overline{11}$ & & & & & & & & . & 2 & 1 & & \\
\hline & S-2 Fan & 1 & 1 & & & 1 & 1 & 1 & 1 & 1 & & & & & & 1 & 2 & 1 & 1 & 1 \\
\hline & S-3 Fan & 1 & 1 & & & 1 & 1 & 1 & & & 1 & & 1 & & 1 & 1 & 2 & & & \\
\hline & S-4 Fan & 1 & 1 & & & 1 & 1 & 1 & & 1 & & & & 1 & & & 1 & & & \\
\hline & S-5 Fan & 1 & 1 & & & 1 & 1 & 1 & & & 1 & & 1 & & 1 & 1 & 2 & & & \\
\hline \multirow{5}{*}{ Library IV } & 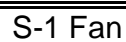 & & 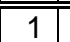 & 1 & 1 & 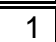 & & & & $\overline{11}$ & & 1 & & & 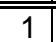 & 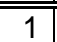 & 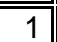 & 1 & & \\
\hline & S-2 Fan & & 1 & 1 & 1 & 1 & & & & 1 & & 1 & & & 1 & 1 & 2 & 1 & & \\
\hline & S-3 Fan & & 1 & & 1 & & 1 & & & & & & & 1 & & & 1 & 1 & & \\
\hline & S-4 Fan & & 1 & & 1 & & 1 & & & & & & & 1 & & & 1 & 1 & & \\
\hline & S-5 Fan & & 1 & & 1 & & 1 & & & & & & & 1 & & & 1 & 1 & & \\
\hline \multicolumn{2}{|c|}{ Total } & 10 & 15 & 2 & 5 & 12 & 13 & 10 & 2 & 9 & 2 & 2 & 2 & 4 & 9 & 10 & 21 & 10 & 4 & 6 \\
\hline
\end{tabular}

\begin{tabular}{|l||l|l|}
\hline Utility Trend & Library II Electrical Demand & $15-$-minute \\
\hline Library II & Library 3 Gas Meter & $15-$ minute \\
\cline { 2 - 4 } & Library III Electrical Demand 1 FI & $15-$ minute \\
\cline { 2 - 4 } & Library III Electrical Demand 8 FI & $15-$ minute \\
\hline Library IV & Library IV Electrical Demand & $15-$ minute \\
\hline \multirow{2}{*}{ Library Main } & Library Chilled Water Use kW & $15-$ minute \\
\cline { 2 - 4 } & Library Main Total & 15 -minute \\
\hline
\end{tabular}

\begin{tabular}{|l||l|l|}
\hline \multirow{5}{*}{ Chiller Trend } & Chilled Water Use & $15-$ minute \\
\cline { 2 - 3 } & Chiller Plant kW & $15-$ minute \\
\cline { 2 - 3 } & Chiller Tons & $15-$ minute \\
\cline { 2 - 3 } & Chilled Water Return Temp & $15-$ minute \\
\cline { 2 - 3 } & Chilled Water Supply Temp & $15-$ minute \\
\cline { 2 - 3 } & Chiller 2 Electrical Demand & $15-$ minute \\
\cline { 2 - 3 } & Chiller 2 Run Status & $15-$ minute \\
\cline { 2 - 3 } & Chiller Full Load Amps & $15-$-minute \\
\cline { 2 - 3 } & Condenser Water Return Temp & $15-$-minute \\
\cline { 2 - 3 } & Condenser Water Supply Temp & $15-$ minute \\
\hline
\end{tabular}





\section{Appendix VII. Additional Result Tables and Figures}

\section{Appendix VII-1. First Day Test Results}

We analyzed the first day test by the whole-building method. Table VIII-1 shows the demand savings for each fifteen minute time period derived using the whole-building method. Since GSA participation failed for the first day test, the other four sites are described here. The maximum aggregated savings was $260 \mathrm{~kW}$. These savings were $11 \%$ of total $2191 \mathrm{~kW}$ demand and $0.30 \mathrm{~W} / \mathrm{ft}^{2}$. Roche achieved the largest demand saving percentage among the four sites (23\%), followed by Albertsons (maximum $16 \%$ of demand saving) and UCSB (maximum $11 \%$ of demand saving). Demand saving was not identified for Bank of America for this test.

Three sites out of four failed some of their shed strategies. Roche achieved all the shed strategies planned. Albertsons failed one of two strategies. UCSB failed one in three strategies. Based on the equipment trend data, Bank of America failed one of one strategy.

Table VII-12. Summary of Estimated Savings of 1st Day (Whole-Building Method)

\begin{tabular}{|c|c|c|c|c|c|c|c|c|c|c|c|c|c|c|c|c|c|}
\hline Time & from & $\begin{array}{l}13: 00 \\
13 \cdot 15\end{array}$ & $\begin{array}{l}13: 15 \\
13 \cdot 3\end{array}$ & $\begin{array}{l}3: 30 \\
3.45\end{array}$ & & $14: 00$ & $\begin{array}{l}14: 15 \\
14 \cdot 3 ?\end{array}$ & $14: 30$ & $\begin{array}{l}14: 45 \\
15 \cdot 0\end{array}$ & $\begin{array}{l}15: 00 \\
15 \cdot 15\end{array}$ & \begin{tabular}{|l|}
$15: 15$ \\
$15 \cdot 30$
\end{tabular} & $\begin{array}{l}15: 30 \\
15 \cdot 45\end{array}$ & $\begin{array}{l}15: 45 \\
16.00\end{array}$ & $\begin{array}{l}16: 00 \\
16 \cdot 15\end{array}$ & \begin{tabular}{|l|}
$16: 15$ \\
$16 \cdot 3$
\end{tabular} & $\begin{array}{l}16: 30 \\
16.45\end{array}$ & 16:45 \\
\hline Price signal & $\$ / \mathrm{kW}$ & \multicolumn{2}{|c|}{0.10} & \multicolumn{5}{|c|}{0.30} & \multicolumn{7}{|c|}{0.75} & \\
\hline \multirow[t]{4}{*}{ Albertsons } & $\overline{\mathrm{BP} k W}$ & 311 & 294 & 305 & 311 & 300 & 302 & 299 & 309 & 303 & 288 & 315 & 327 & 305 & 312 & 314 & 327 \\
\hline & Shed & 37 & 53 & 54 & 52 & 54 & 42 & 48 & 51 & 51 & 54 & 33 & 36 & 50 & & 38 & \\
\hline & ned \% & $11 \%$ & $15 \%$ & $15 \%$ & $14 \%$ & $15 \%$ & $12 \%$ & $14 \%$ & $14 \%$ & $14 \%$ & $16 \%$ & $10 \%$ & $10 \%$ & $14 \%$ & $11 \%$ & $11 \%$ & $9 \%$ \\
\hline & /spft & 0.73 & 1.05 & 1.09 & 1.04 & 1.08 & 0.84 & 0.96 & 1.02 & 1.02 & 1.07 & 0.67 & 0.72 & 1.01 & .79 & .77 & .67 \\
\hline \multirow{4}{*}{$\begin{array}{l}\text { Bank of } \\
\text { America }\end{array}$} & N & 735 & 744 & 734 & 733 & 735 & 731 & 74 & 724 & 730 & 760 & 729 & 720 & 01 & 93 & 80 & 63 \\
\hline & & -13 & -16 & -8 & -2 & -4 & -8 & -19 & -5 & -10 & -42 & -11 & -12 & -1 & & 1 & \\
\hline & ed $\%$ & $-2 \%$ & $-2 \%$ & $-1 \%$ & $0 \%$ & $-1 \%$ & $-1 \%$ & $-3 \%$ & $-1 \%$ & $-1 \%$ & $-6 \%$ & $-2 \%$ & $-2 \%$ & $0 \%$ & $0 \%$ & $0 \%$ & $0 \%$ \\
\hline & /spft & -0.06 & -0.08 & -0.04 & -0.01 & -0.02 & -0.04 & -0.09 & -0.03 & -0.05 & -0.20 & -0.05 & -0.05 & \begin{tabular}{|l|}
-0.01 \\
\end{tabular} & 0.00 & 0.01 & 0.00 \\
\hline \multirow[t]{4}{*}{ Roche } & $\mathrm{W}$ & 702 & 662 & 568 & 639 & 551 & 585 & 464 & 455 & 452 & 447 & 439 & 431 & 424 & 577 & 464 & $\overline{455}$ \\
\hline & $N$ & -31 & 9 & 79 & 1 & 73 & 27 & 123 & 31 & 131 & 9 & 130 & 123 & 124 & & 26 & 1 \\
\hline & ed \% & $-5 \%$ & $1 \%$ & $12 \%$ & $\%$ & $12 \%$ & $4 \%$ & $21 \%$ & $22 \%$ & $22 \%$ & $2 \%$ & $23 \%$ & $22 \%$ & $23 \%$ & $-6 \%$ & $5 \%$ & $4 \%$ \\
\hline & Shed W/spft & -0.16 & 0.05 & 0.41 & 0.01 & 0.38 & 0.14 & 0.64 & 0.68 & 0.68 & 0.67 & 0.68 & 0.64 & 0.64 & -0.18 & 0.14 & 0.10 \\
\hline \multirow[t]{4}{*}{ UCSB } & WBP kW & 779 & 797 & 794 & 801 & 786 & 798 & 807 & 703 & 706 & 706 & 697 & 712 & 716 & 866 & 775 & $\overline{744}$ \\
\hline & & 22 & 3 & 2 & -7 & 3 & -6 & -19 & 83 & 66 & 77 & 84 & 60 & 61 & 72 & 4 & \\
\hline & ed \% & $3 \%$ & $0 \%$ & $0 \%$ & $-1 \%$ & $0 \%$ & $-1 \%$ & $-2 \%$ & $\%$ & $9 \%$ & $10 \%$ & $11 \%$ & $8 \%$ & $8 \%$ & $f$ & $1 \%$ & $4 \%$ \\
\hline & Shed W/spft & 0.08 & 0.01 & 0.01 & -0.02 & 0.01 & -0.02 & -0.06 & 0.29 & 0.23 & 0.26 & 0.29 & 0.21 & 0.21 & -0.25 & 0.01 & 0.12 \\
\hline \multirow[t]{4}{*}{ Total } & WBP kW & 2527 & 2497 & 2400 & 2483 & 2371 & 2416 & 2311 & 2191 & 2191 & 2201 & 2180 & 2191 & 2145 & 2448 & 2232 & $\overline{2193}$ \\
\hline & & 15 & 48 & 127 & 44 & 126 & 56 & 133 & 260 & 238 & 217 & 236 & 207 & 234 & -6 & 71 & 87 \\
\hline & ed $\%$ & $1 \%$ & $2 \%$ & $5 \%$ & $2 \%$ & $5 \%$ & $2 \%$ & $5 \%$ & $11 \%$ & $10 \%$ & $9 \%$ & $10 \%$ & $9 \%$ & $10 \%$ & -39 & $3 \%$ & $4 \%$ \\
\hline & Shed W/spft & 0.02 & 0.06 & 0.17 & 0.06 & 0.17 & 0.08 & 0.18 & 0.35 & 0.32 & 0.29 & 0.32 & 0.28 & 0.31 & -0.09 & 0.10 & 0.12 \\
\hline
\end{tabular}




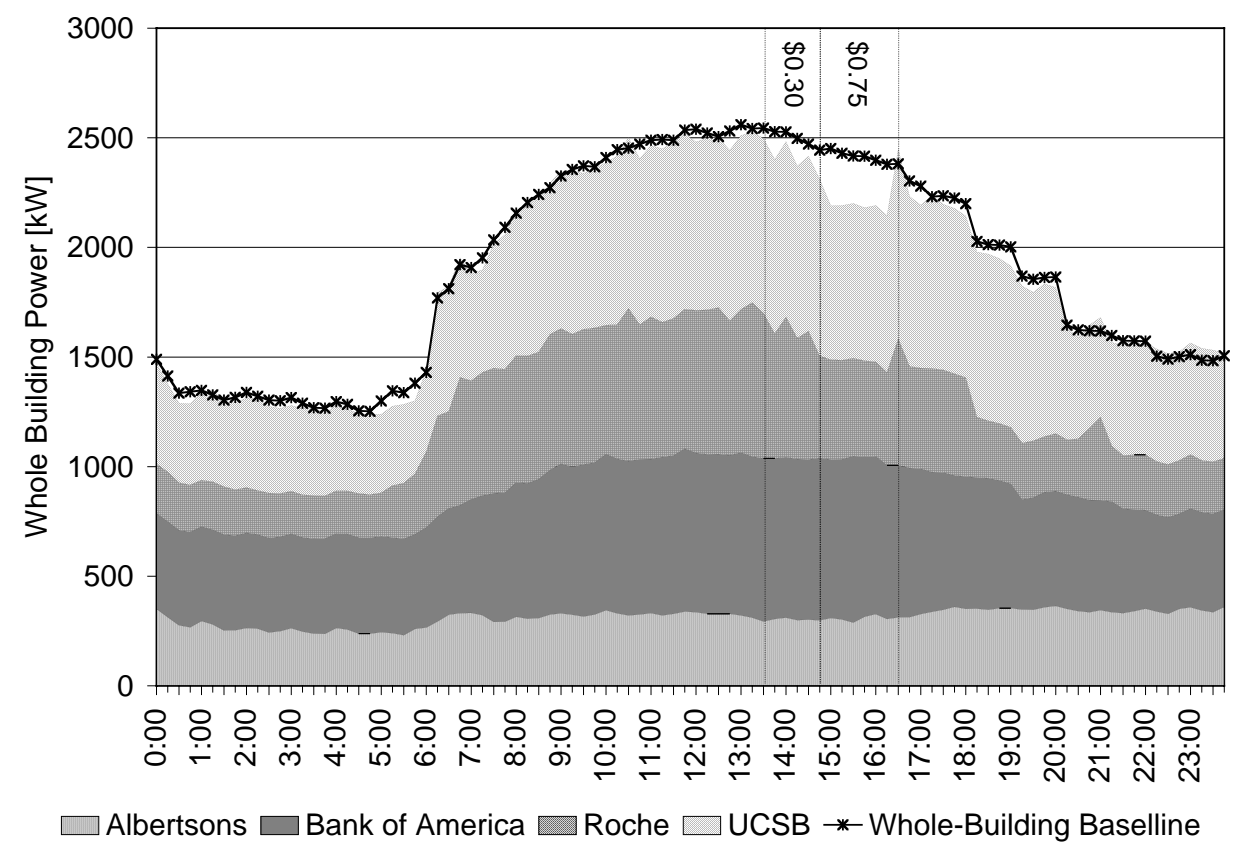

Figure VII-2. Summary of All Sites (1st Day)

Albertsons implemented only their overhead light shed for the first day test. Although the whole-building method indicates maximum $54 \mathrm{~kW}$ of savings, the component level analysis shows $26 \mathrm{~kW}$ of savings in the overhead light. The EIS was programmed to disable the shed (stop polling the signal) at 3:30 pm due to the client's request. The shed was disabled at 3:30 pm, and the overhead lights came back on. However, the shed activated again at 4:00 pm, and lasted until the end of the high price signal (the cause hasn't been identified). The disable program was removed for the second day test.

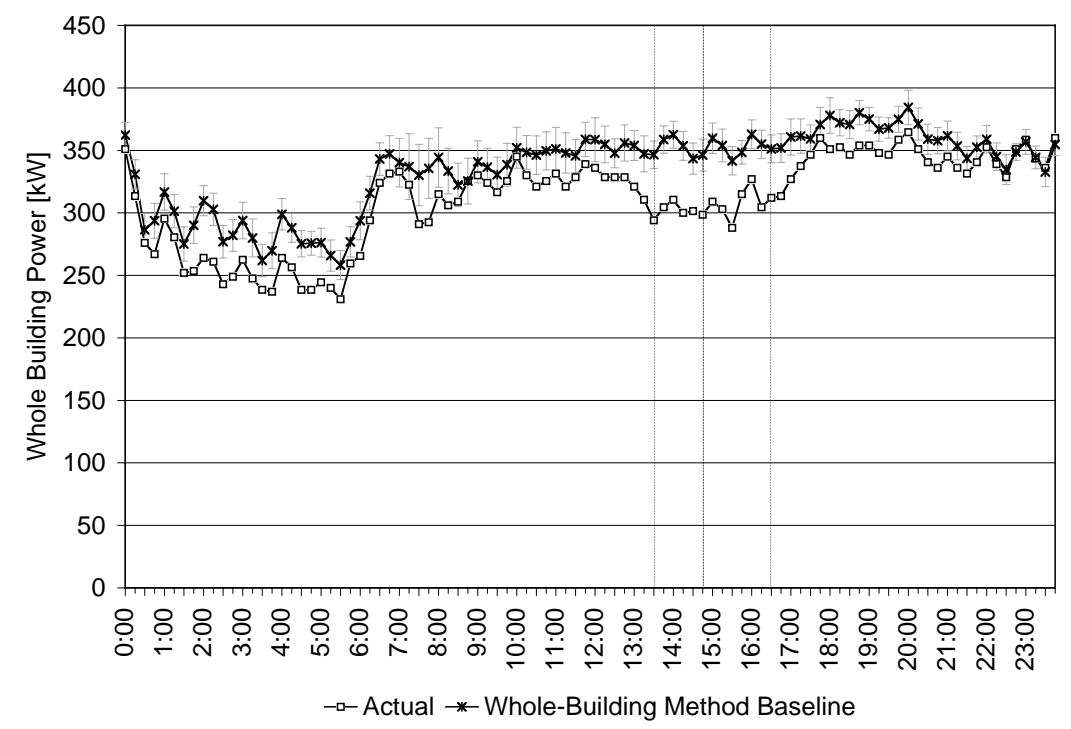

Figure VII-3. Albertsons - Whole Building Power and Baseline (1st Day) 
Bank of America didn't show any noticeable saving. WebGen trend didn't show any changes in the static pressure either.

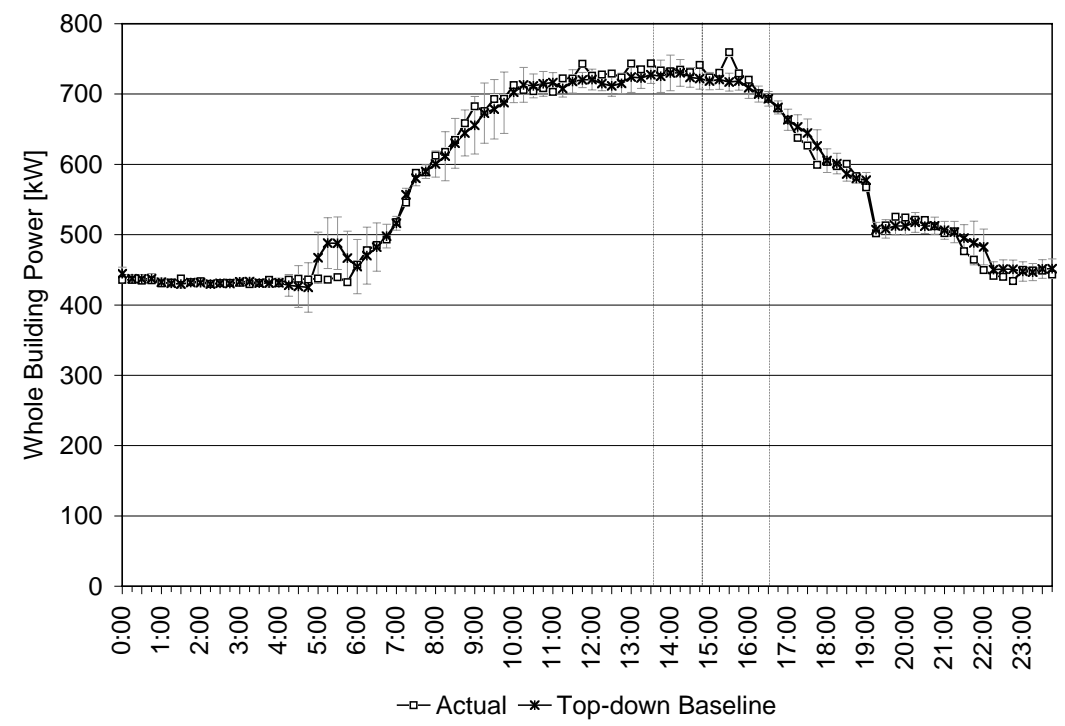

Figure VII-4. B of A - Whole Building Power and Baseline (1st Day)

Roche achieved a complete set of the shed strategies. Though the savings during the $\$ 0.30 / \mathrm{kWh}$ period is not clear because of the cafeteria's inconsistent load, the Building A2 individual building power trend clearly showed $85 \mathrm{~kW}$ of demand saving. During the $\$ 0.75 / \mathrm{kWh}$ period, Roche achieved approximately $130 \mathrm{~kW}$ of demand saving. The electricity demand spiked at the end of shed period, probably because of release of the equipment operations.

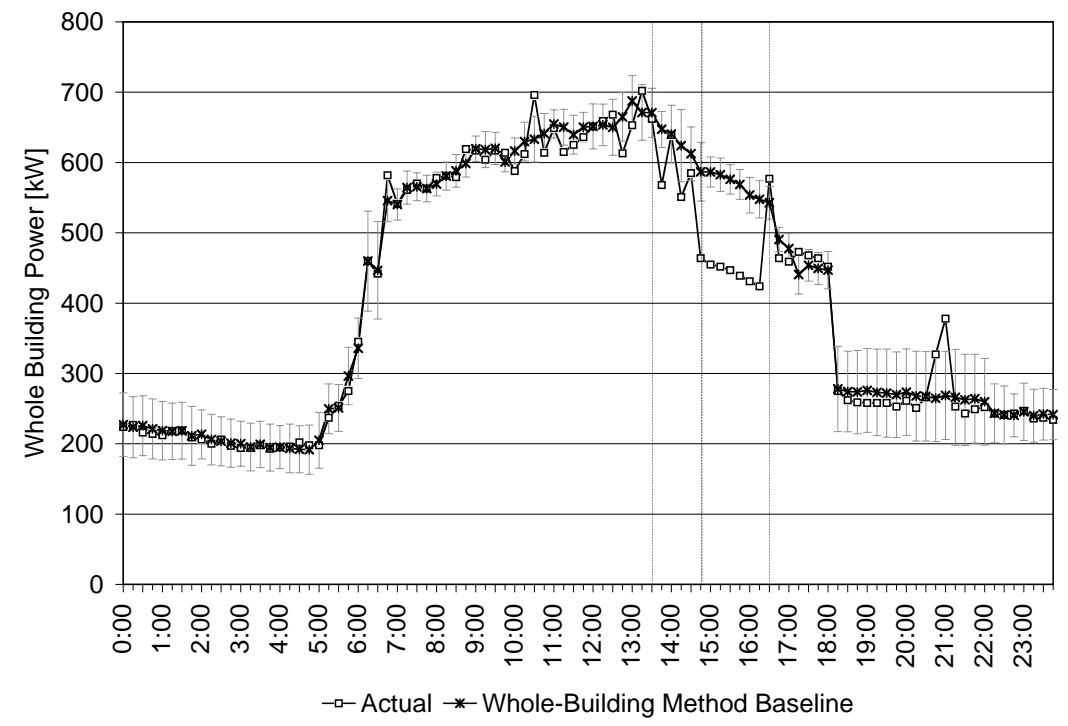

Figure VII-5. Roche - Whole Building Power and Baseline (1st Day) 
UCSB failed to operate the fan VFD limit strategy. Therefore, no fan VFD-related savings could be identified during the $\$ 0.30 / \mathrm{kWh}$ period. UCSB succeeded with the cooling valve shed, and the whole-building method indicates a maximum savings of 84 $\mathrm{kW}$. Because of release of the cooling valve, the cooling power spiked after the shed period.

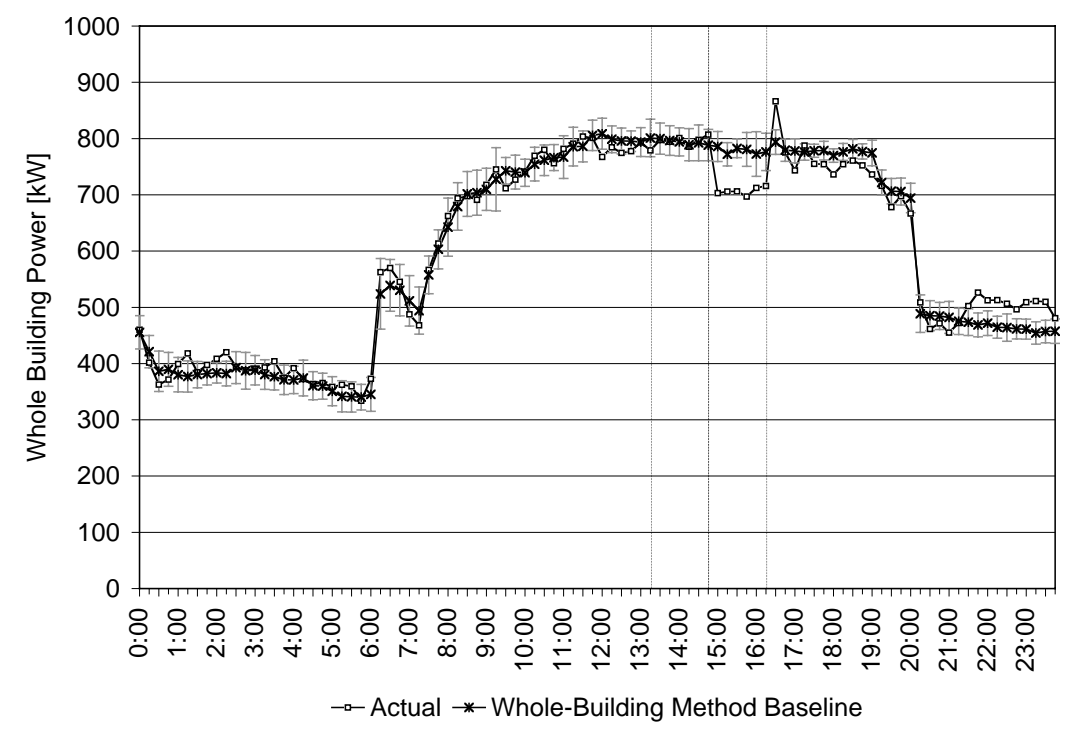

Figure VII-6. UCSB - Whole Building Power and Baseline (1st Day) 


\section{Appendix VII-2. B of A - Result Analysis by WebGen}

WebGen's analysts independently calculated the demand savings at the B of A site using their own method. According to their calculations, Building B achieved maximum savings of $26.4 \mathrm{~kW}$, and the maximum demand saving for the whole site (including the four buildings) was $199 \mathrm{~kW}$ (23\% of the whole building power). The average demand savings for the whole site during the shed period was $8.3 \%$. The WebGen's agents rotated the static pressure reset strategy across the three buildings to minimize the shed impact. The shed rotation can be clearly seen in Table VIII-3.

Table VII-13. B of A - Duct Static Pressure Change

\begin{tabular}{|c|c|c|c|c|}
\hline Time & Bldg A & Bldg B & Bldg C & Min SP \\
\hline $12: 45$ & 2.21 & 2.20 & 2.18 & 2.18 \\
\hline $13: 00$ & 1.95 & 2.19 & 2.19 & 1.95 \\
\hline $13: 15$ & 2.29 & 2.19 & 2.23 & 2.19 \\
\hline $13: 30$ & 2.17 & 1.98 & 2.25 & 1.98 \\
\hline $13: 45$ & 1.65 & 1.99 & 2.23 & 1.65 \\
\hline $14: 00$ & 1.88 & 2.20 & 2.22 & 1.88 \\
\hline $14: 15$ & 2.15 & 2.22 & 2.20 & 2.15 \\
\hline $14: 30$ & 2.25 & 2.01 & 2.17 & 2.01 \\
\hline $14: 45$ & 2.03 & 2.20 & 2.16 & 2.03 \\
\hline $15: 00$ & 1.85 & 2.19 & 2.15 & 1.85 \\
\hline $15: 15$ & 2.24 & 2.20 & 1.74 & 1.74 \\
\hline $15: 30$ & 2.37 & 2.00 & 2.19 & 2.00 \\
\hline $15: 45$ & 2.41 & 1.98 & 2.25 & 1.98 \\
\hline $16: 00$ & 1.99 & 2.19 & 2.25 & 1.99 \\
\hline $16: 15$ & 2.09 & 2.20 & 2.08 & 2.08 \\
\hline $16: 30$ & 2.37 & 2.22 & 2.24 & 2.22 \\
\hline $16: 45$ & 2.41 & 2.19 & 2.20 & 2.19 \\
\hline $17: 45$ & 2.41 & 2.20 & 2.19 & 2.19 \\
\hline $18: 45$ & 2.38 & 2.21 & 2.21 & 2.21 \\
\hline
\end{tabular}

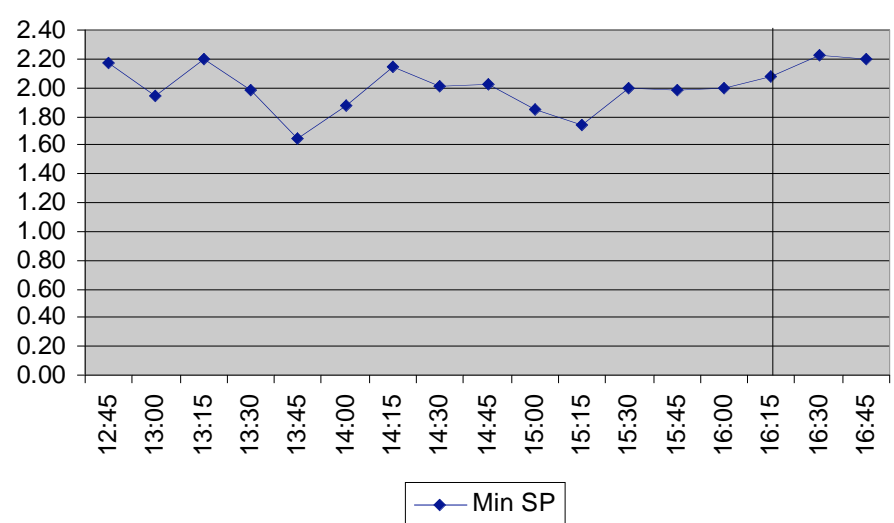

Figure VII-7. B of A - Duct Static Pressure 
Table VII-14. B of A - Whole Building Power Saving Estimates by WebGen

\begin{tabular}{|c|c|c|c|c|c|}
\hline \multirow{2}{*}{ Time } & \multicolumn{3}{|c|}{$\Delta \mathrm{kW}$} & \multirow{2}{*}{ Sum $\Delta \mathrm{kW}$} & \multirow{2}{*}{$\%$ of Mtr } \\
\hline & Bldg A & Bldg B & BIdg C & & \\
\hline $12: 45$ & 0.00 & 0.19 & 2.99 & 3.18 & $0.7 \%$ \\
\hline $13: 00$ & 46.77 & 1.69 & 1.97 & 50.44 & $9.8 \%$ \\
\hline $13: 15$ & 0.00 & 1.04 & 0.00 & 1.04 & $0.2 \%$ \\
\hline $13: 30$ & 6.22 & 26.40 & 0.00 & 32.62 & $6.3 \%$ \\
\hline $13: 45$ & 94.09 & 25.18 & 0.00 & 119.27 & $23.1 \%$ \\
\hline $14: 00$ & 57.82 & 0.22 & 0.00 & 58.04 & $11.3 \%$ \\
\hline $14: 15$ & 10.53 & 0.00 & 0.69 & 11.22 & $2.2 \%$ \\
\hline $14: 30$ & 0.00 & 23.26 & 4.10 & 27.36 & $5.3 \%$ \\
\hline $14: 45$ & 27.38 & 0.00 & 5.43 & 32.81 & $7.6 \%$ \\
\hline $15: 00$ & 44.68 & 0.85 & 5.60 & 51.13 & $14.0 \%$ \\
\hline $15: 15$ & 0.00 & 0.38 & 49.22 & 49.60 & $11.9 \%$ \\
\hline $15: 30$ & 0.00 & 19.30 & 0.66 & 19.97 & $4.9 \%$ \\
\hline $15: 45$ & 0.00 & 18.60 & 0.00 & 18.60 & $5.0 \%$ \\
\hline $16: 00$ & 20.30 & 0.88 & 0.00 & 21.19 & $7.9 \%$ \\
\hline $16: 15$ & 10.82 & 0.05 & 9.12 & 20.00 & $7.4 \%$ \\
\hline $16: 30$ & 0.00 & 0.00 & 0.00 & 0.00 & $0.0 \%$ \\
\hline $16: 45$ & 0.00 & 0.36 & 0.00 & 0.36 & $0.1 \%$ \\
\hline $17: 45$ & 0.00 & 0.00 & 0.66 & 0.66 & $0.3 \%$ \\
\hline $18: 45$ & 0.00 & 0.00 & 0.00 & 0.00 & $0.0 \%$ \\
\hline & & & Average & 36.66 & $8.3 \%$ \\
\hline
\end{tabular}

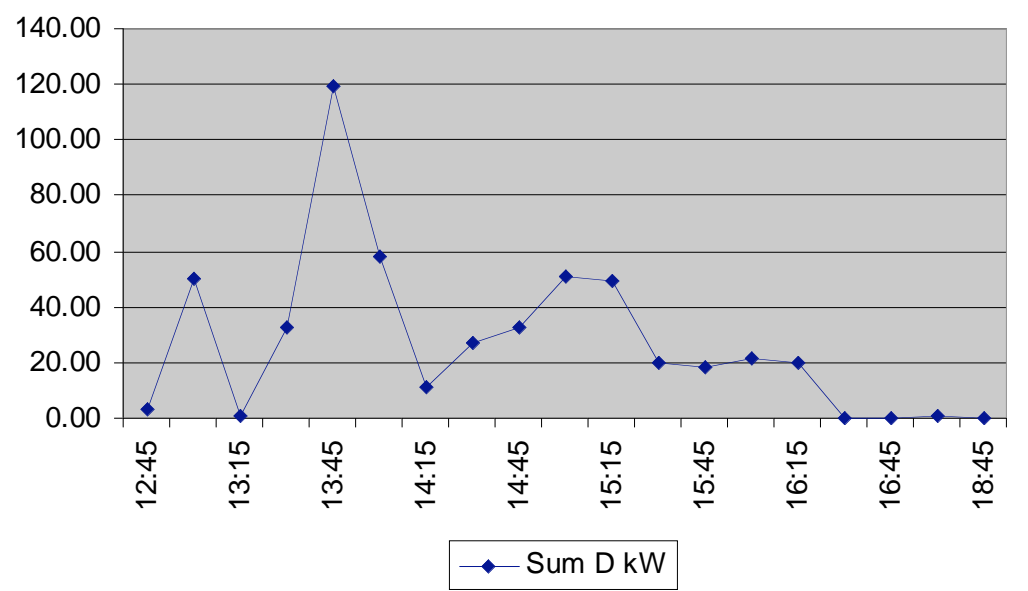

Figure VII-8. B of A - Whole Building Power Saving Estimates by WebGen 


\section{Appendix VII-3. GSA - Additional Figures}

\section{Individual Component Power and Baseline}

Baseline demand for the controllable components were calculated by using the OAT regression method. Because of the "tower free cooling" strategy, the chillers and the related equipment operation show irregular daily load shapes. Chiller demand increased during the first $\$ 0.30 / \mathrm{kWh}$ period, and dropped down at the beginning of the $\$ 0.75 / \mathrm{kWh}$ period (Figure VIII-8).

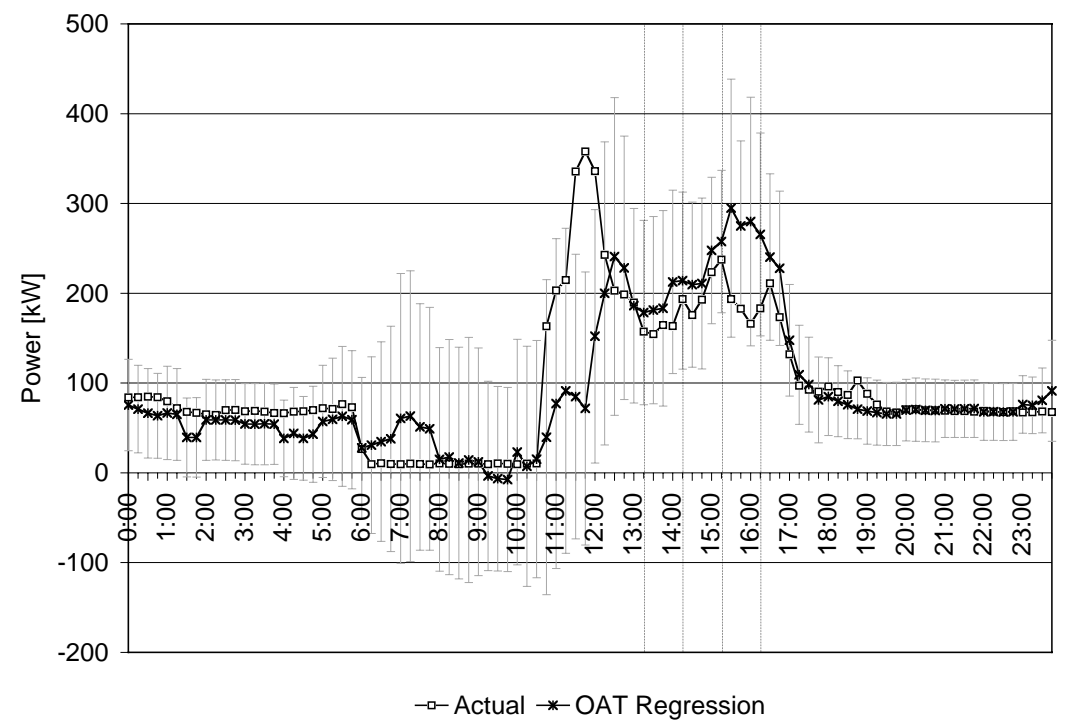

Figure VII-9. GSA - Chiller Power and OAT Regression Model

Fan power shows the demand reduction clearly during the shed. The OAT regression model seems higher than the actual demand (Figure VIII-9).

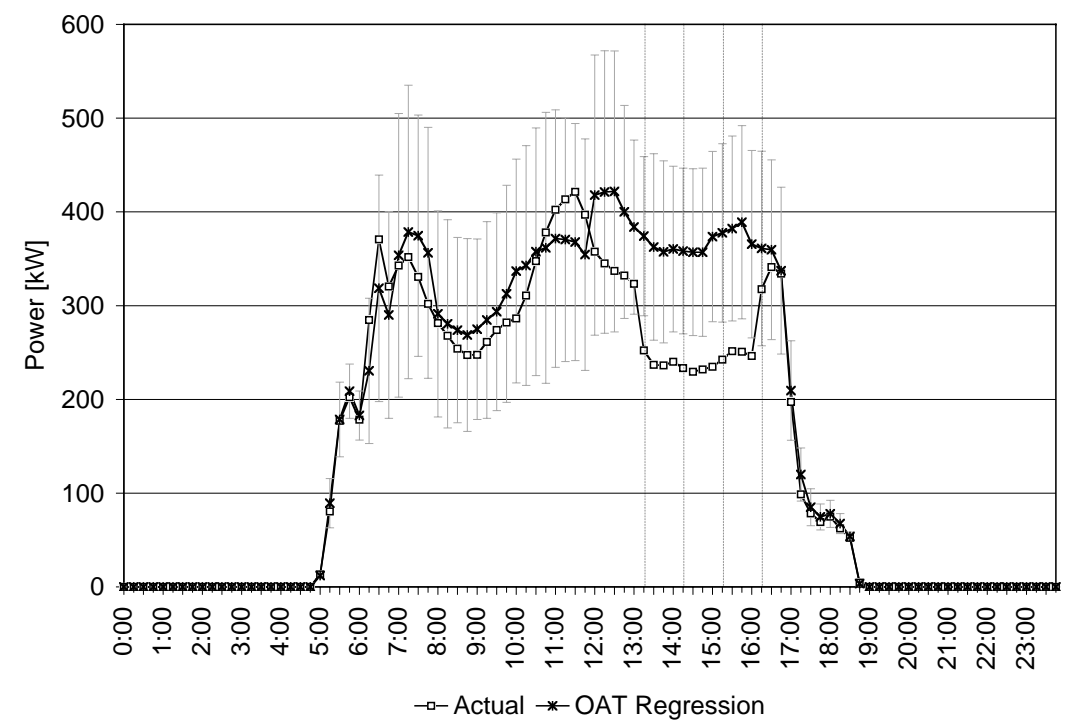

Figure VII-10. GSA - Fan Power and OAT Regression Model 
The pump power showed a demand spike at the end of the $\$ 0.75 / \mathrm{kWh}$ period, due to the start-up of the chillers (Figure VIII-10).

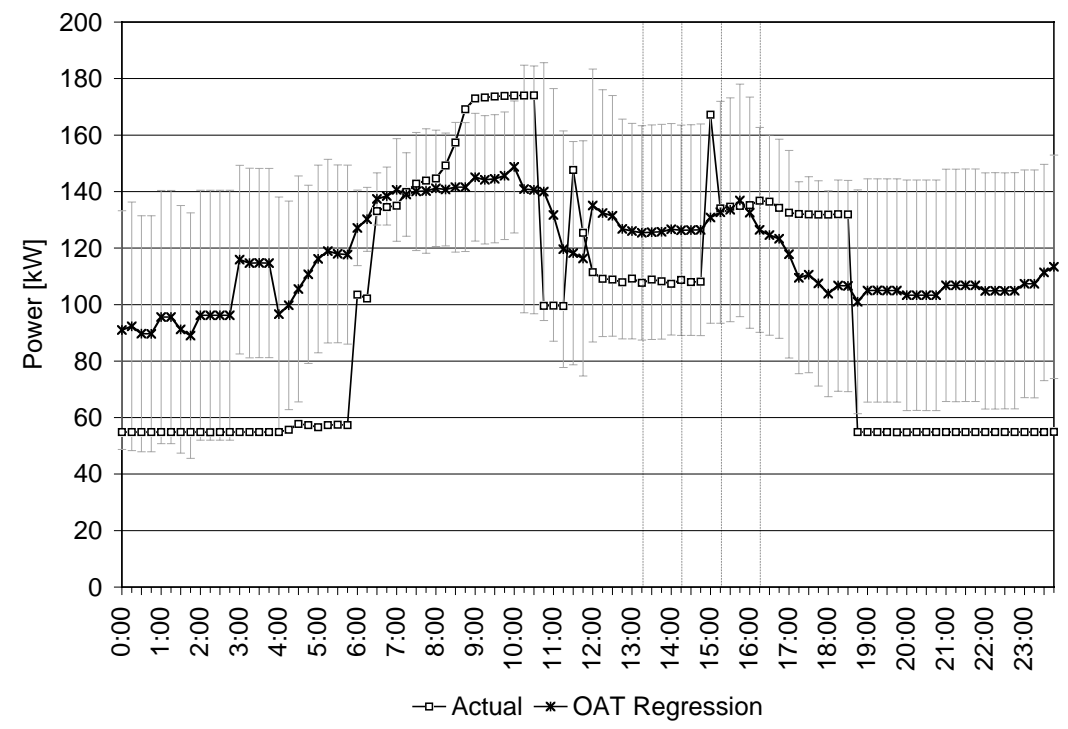

Figure VII-11. GSA - Pump Power and OAT Regression Model

No obvious demand savings could be identified for the cooling tower (Figure VIII-11).

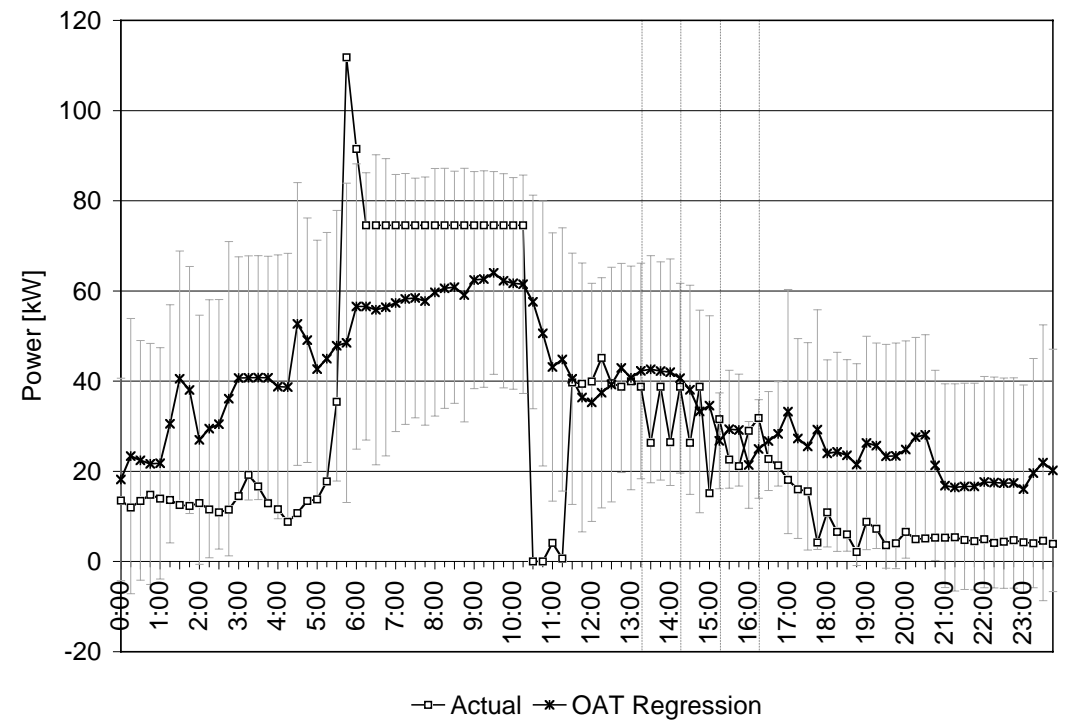

Figure VII-12. GSA - Cooling Tower Power and OAT Regression Model 
The pump power spiked at 3:00 pm due to the chiller staging-up (Figure VIII-12).

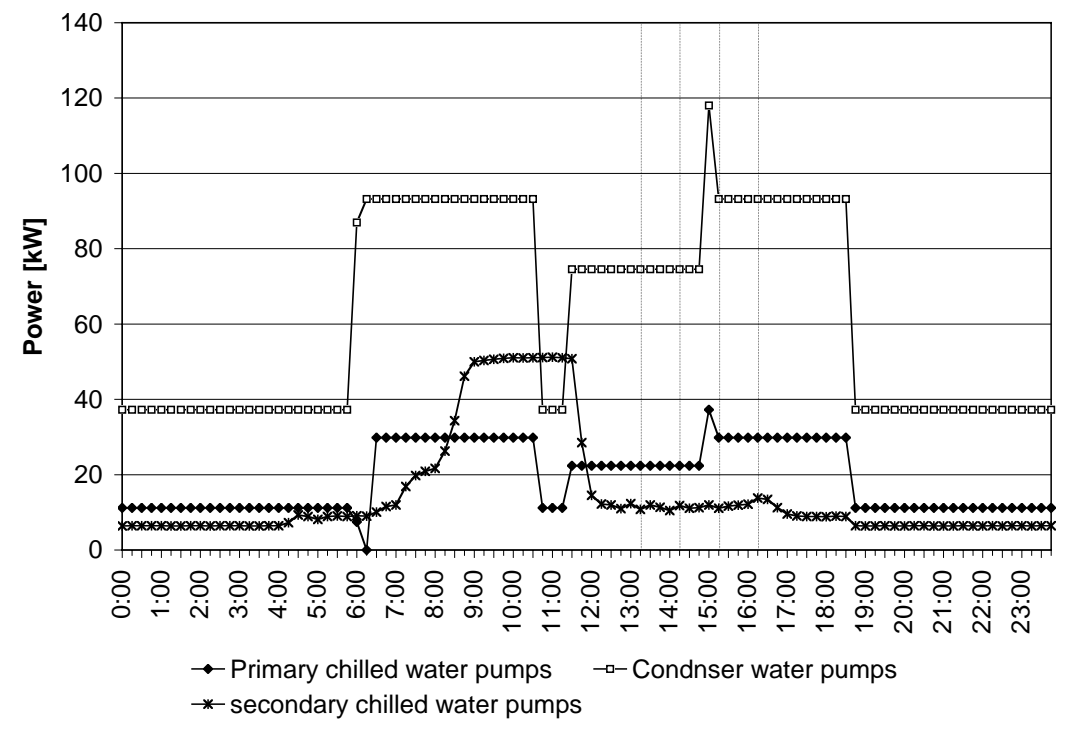

Figure VII-13. GSA - Pump Power

\section{$\underline{\text { Zone Temperatures by Floor }}$}

Zone temperatures were collected on nine floors. Each floor has four to five zones (see point list at Appendix IV). The zone temperature trends for each floor are presented in the following charts (Figures V111-13 through VIII-21). As a general trend, the west facing zone's temperatures rose in the afternoon regardless of the setpoint increase. The North 2nd floor zone temperature didn't show any obvious changes. Zone 2B1 was in heating mode during the test (Figure VIII-13). 


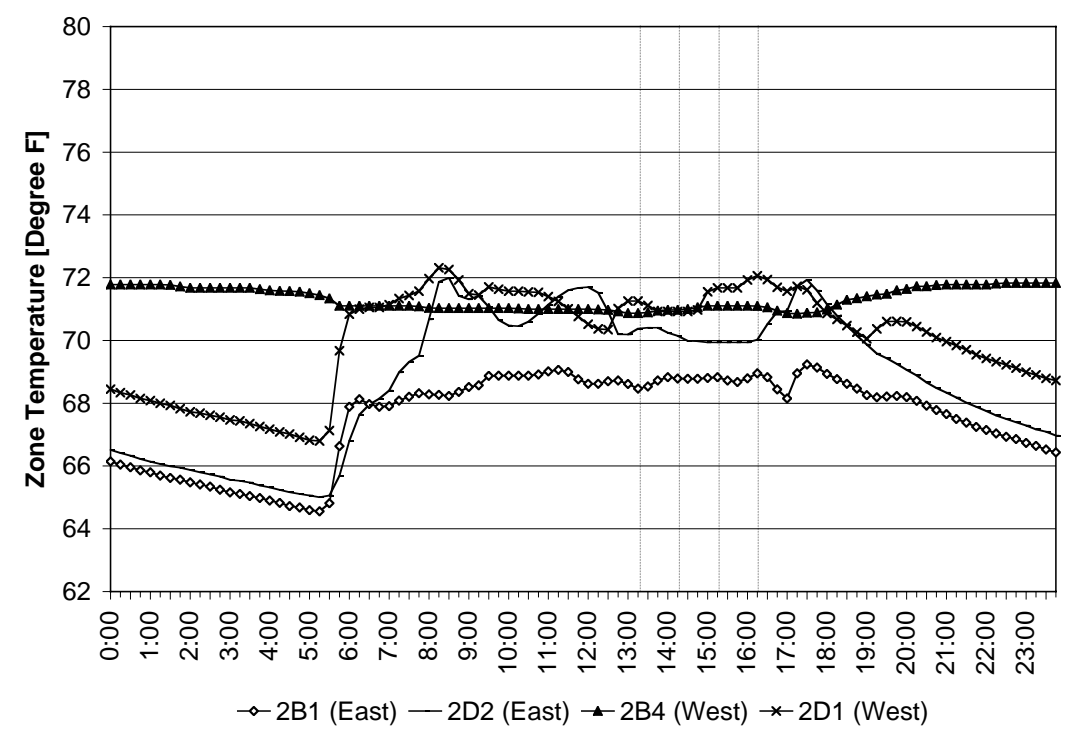

Figure VII-14. GSA - North Tower 2nd Floor Zone Temperature

For the North $4^{\text {th }}$ floor (Figure VIII-14), the zone temperature for the west-facing zone (4D3) increased during the test, possibly because of solar gain. No significant temperature changes were found in the rest of the North $4^{\text {th }}$ floor zones.

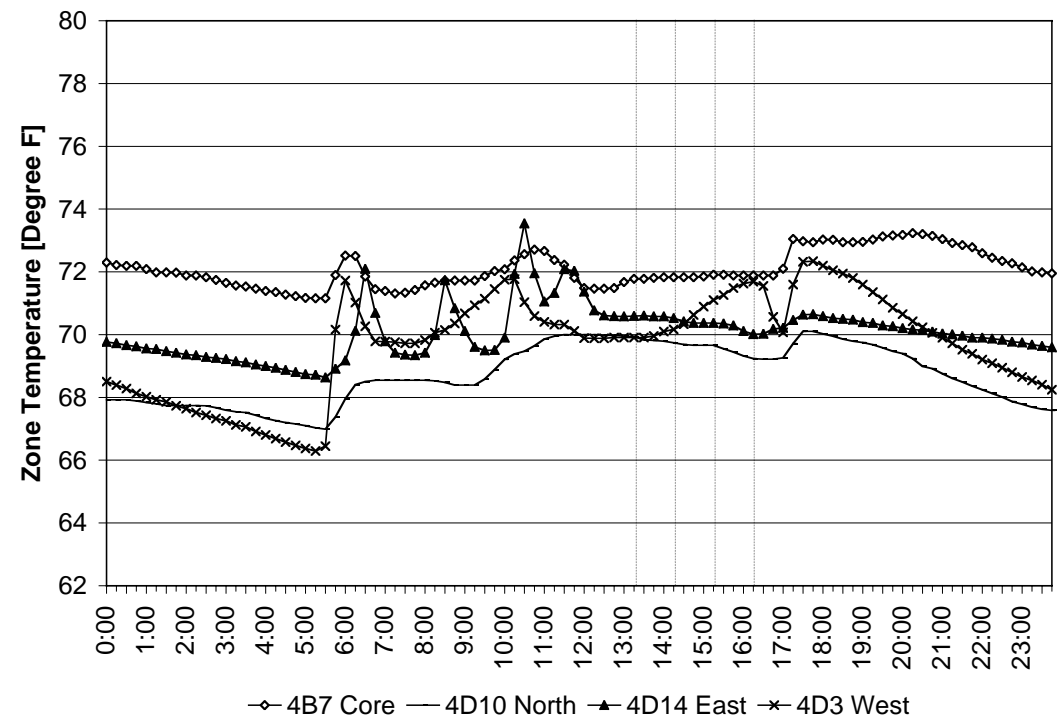

Figure VII-15. GSA - North Tower 4th Floor Zone Temperature

For the North 6th floor (Figure VIII-15), the 6D1 zone temperature dropped during the shed. However, the range of temperatures observed were not around the heating setting point. We are not sure whether the zone was in heating mode or cooling mode. 


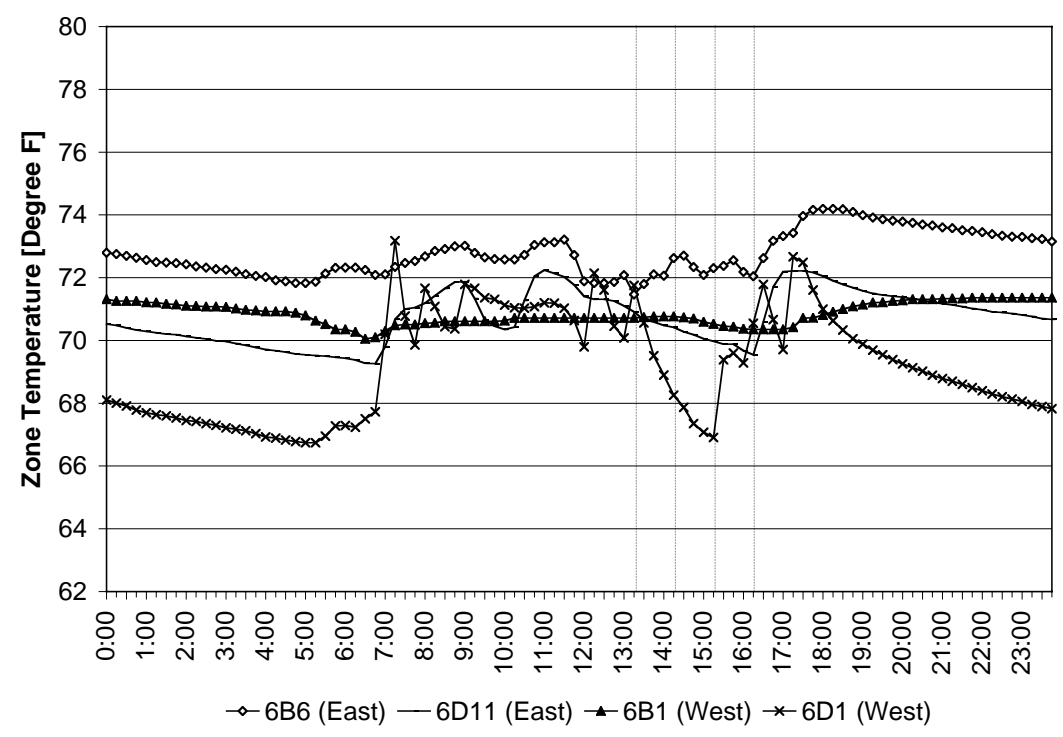

Figure VII-16. GSA - North Tower 6th Floor Zone Temperature 
Most of zone temperatures on North 12th floor increased during the shed (Figure VIII16). However, they changed gradually and didn't show clear setpoint staging based on the price signal.

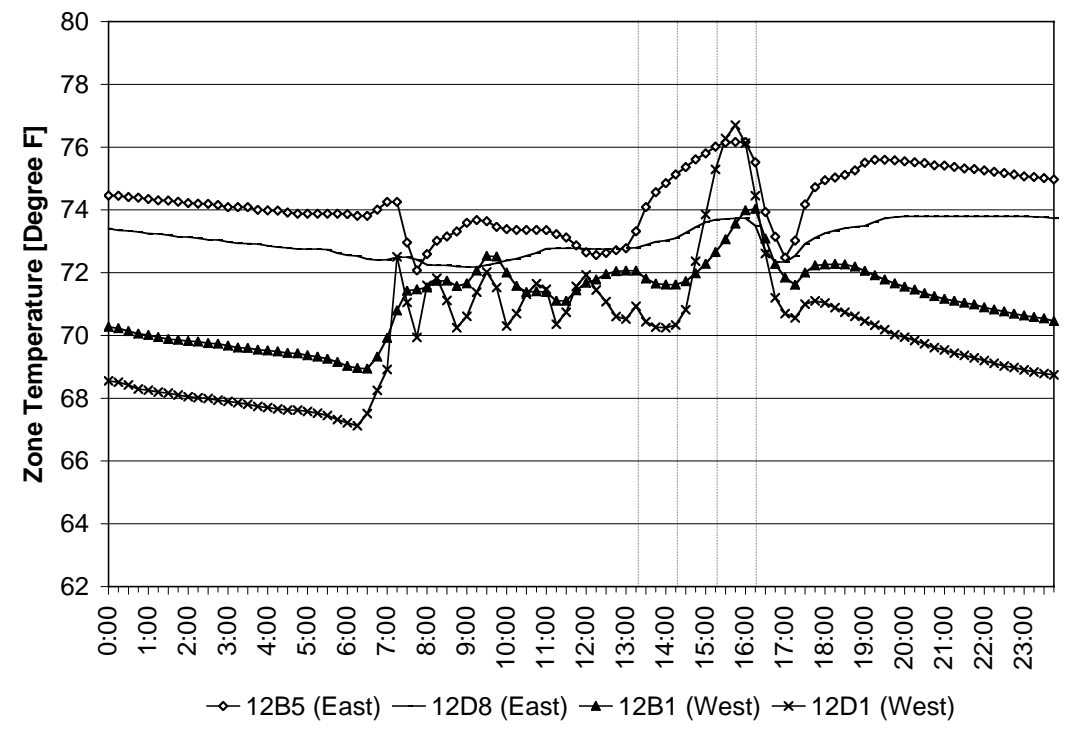

Figure VII-17. GSA - North Tower 12th Floor Zone Temperature

Zone temperatures on the North 16th floor increased during the shed, but didn't reach the assigned setpoint (Figure VIII-17).

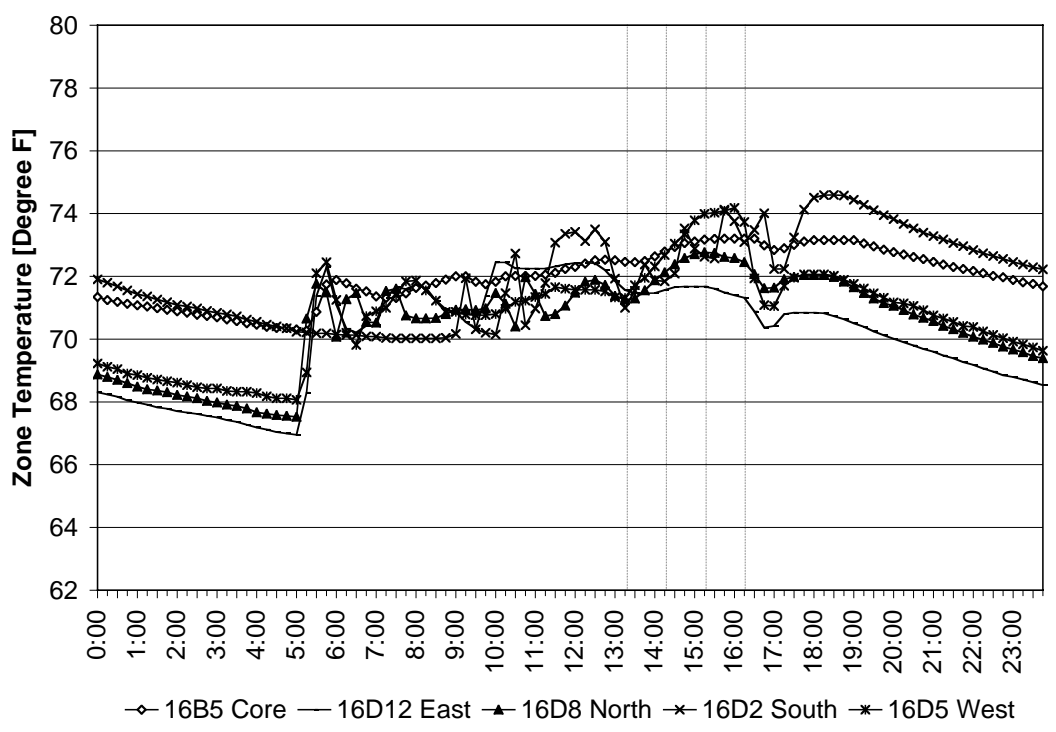

Figure VII-18. GSA - North Tower 16th Floor Zone Temperature 
Zone 17D1's temperature on North 17th floor clearly changed based on the setpoint changes (Figure VIII-18). The rest of the zone temperatures changed gradually. Some of the zone temperatures may have increased because of solar gain.

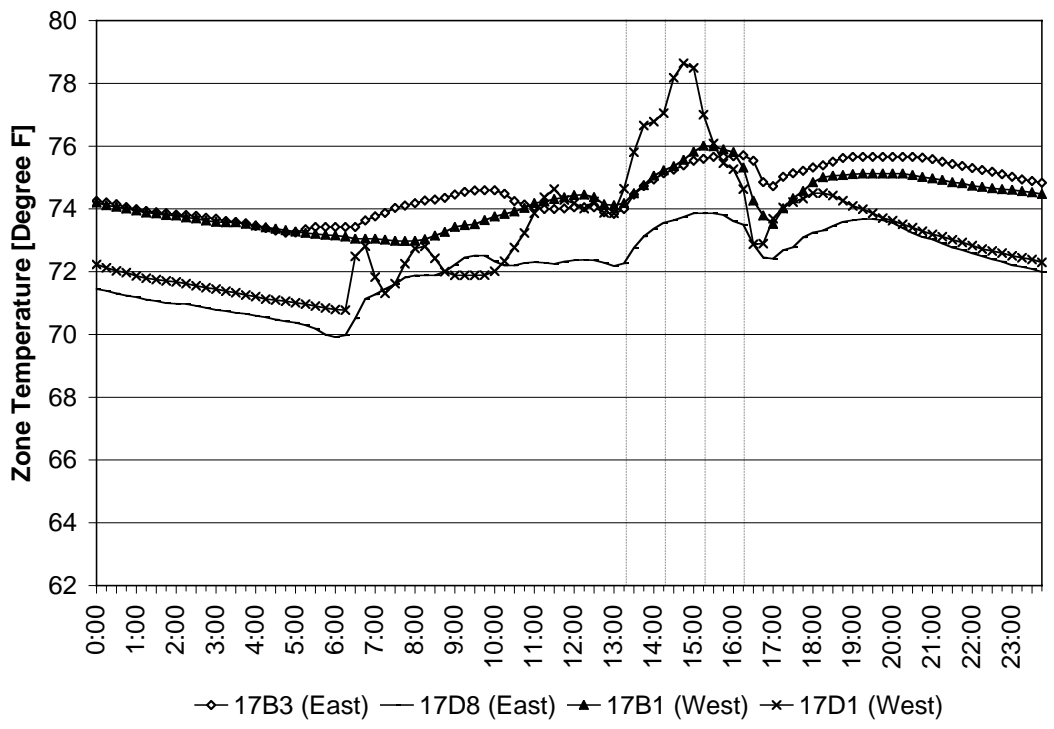

Figure VII-19. GSA - North Tower 17th Floor Zone Temperature

Zone 2D1's temperature on the South 2nd floor increased during the shed (Figure VIII19).

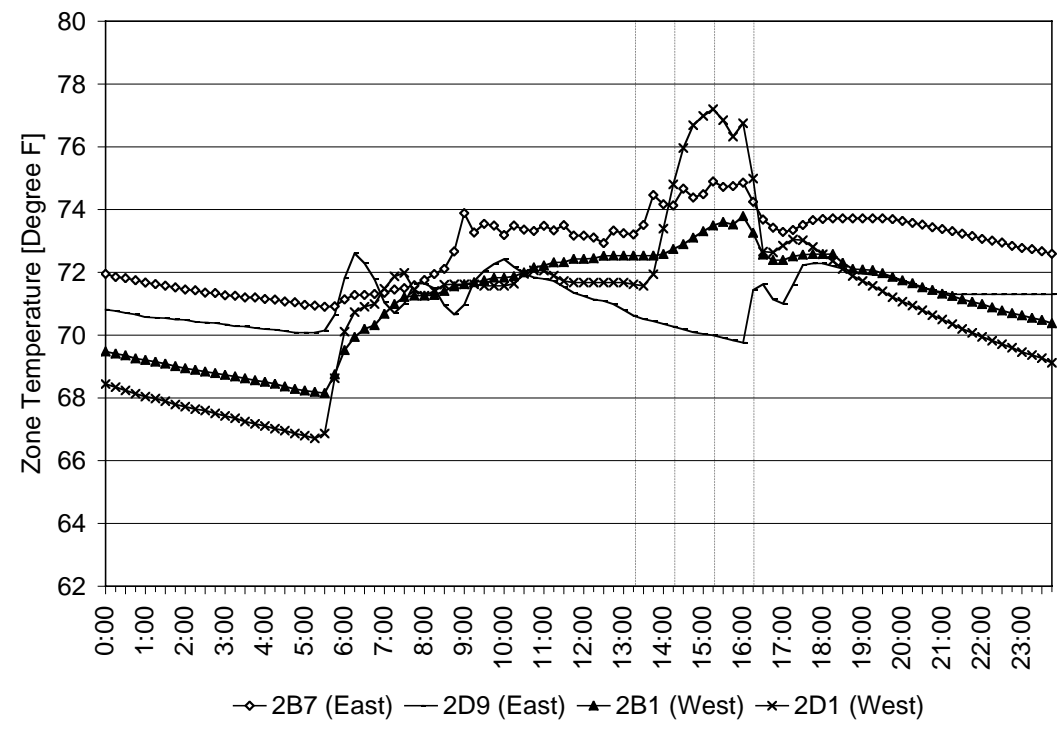

Figure VII-20. $\quad$ GSA - South Tower 2nd Floor Zone Temperature 
Zone temperatures on the South 4th floor did not change based on the price signal (Figure VIII-20). The temperature of the west-facing zone (4D6) increased, possibly due to solar gain.

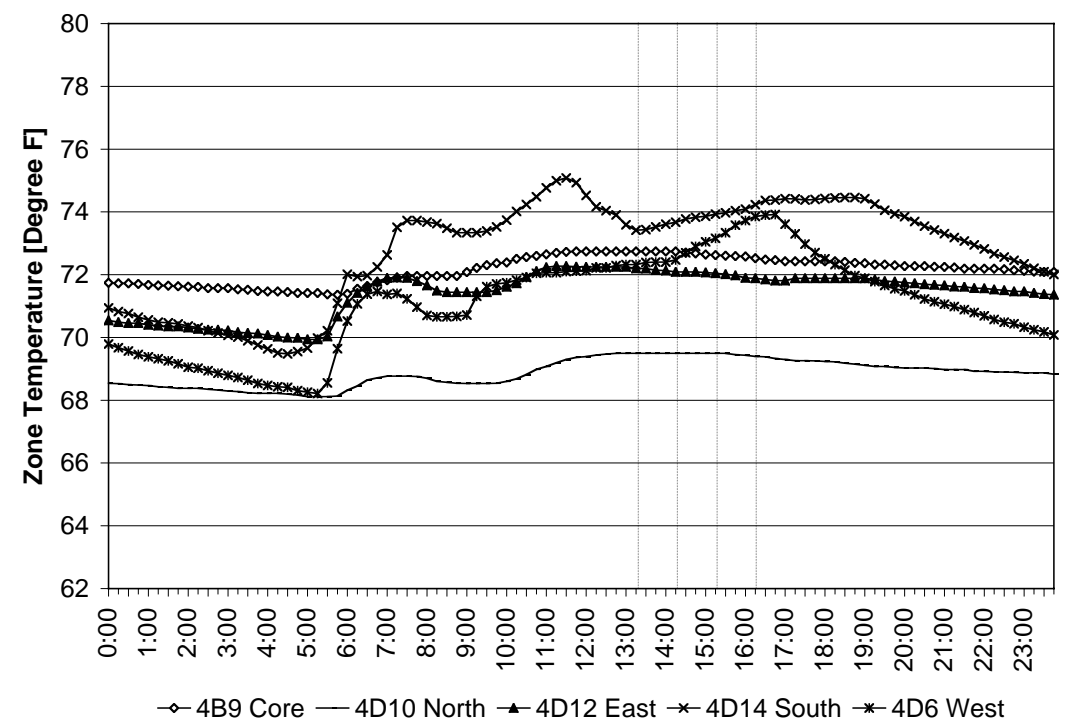

Figure VII-21. GSA - South Tower 4th Floor Zone Temperature

The south-facing zone temperatures on South 16th floor (16D11) clearly changed based on the price signal (Figure VIII-21).

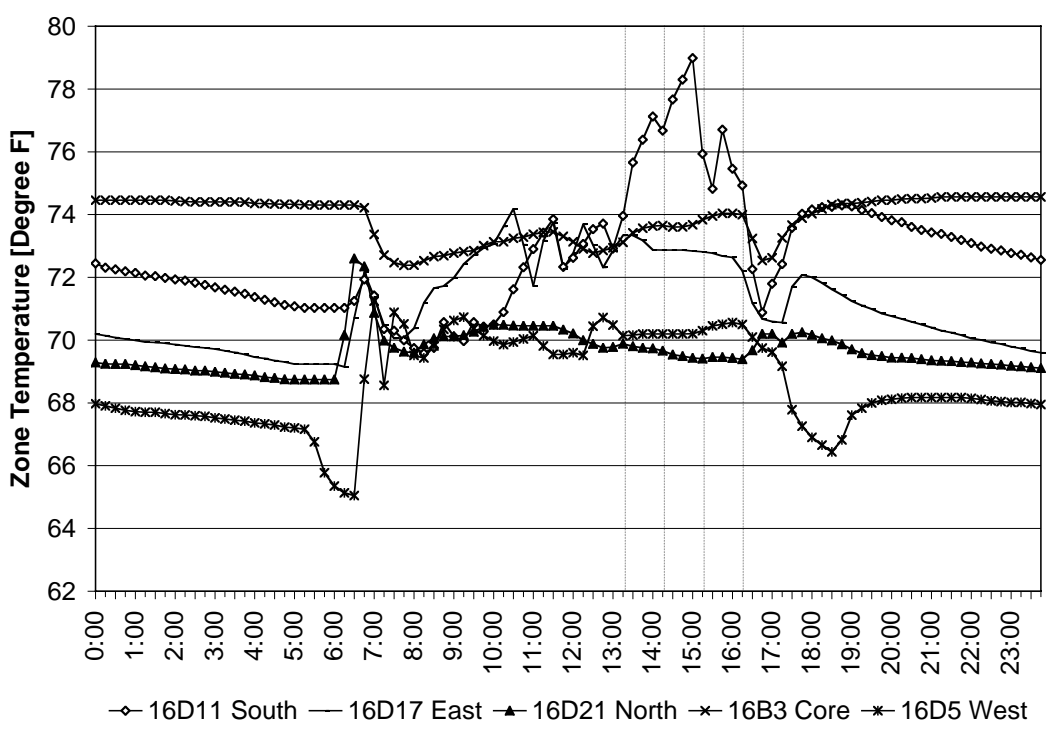

Figure VII-22. GSA - South Tower 16th Floor Zone Temperature 


\section{VAV Damper Positions by Floor}

The VAV damper positions on the North 4th floor were not affected by the price signal, possibly due to the minimum damper position setting (Figure VIII-22).

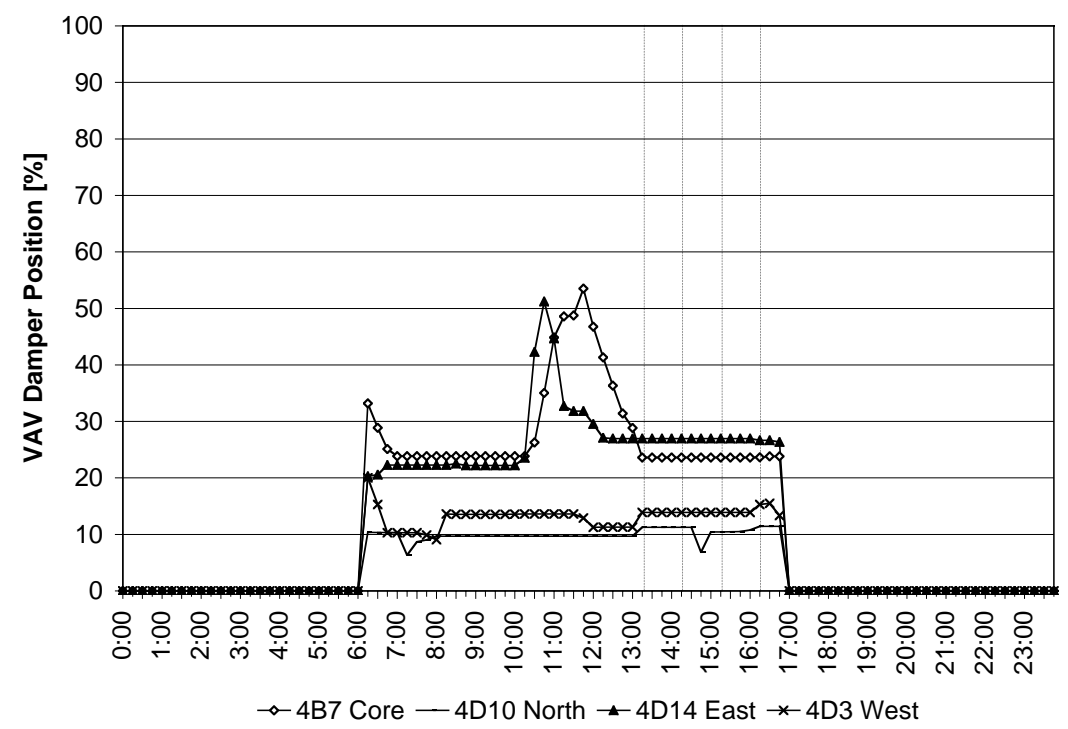

Figure VII-23. GSA - North 4th Floor VAV Damper Position

The VAV damper positions on the North 16th floor seemed not to be affected by the price signal (Figure VIII-23). The PI parameter (proportional integral) setting of the south-side zone (16D8) may need to be calibrated.

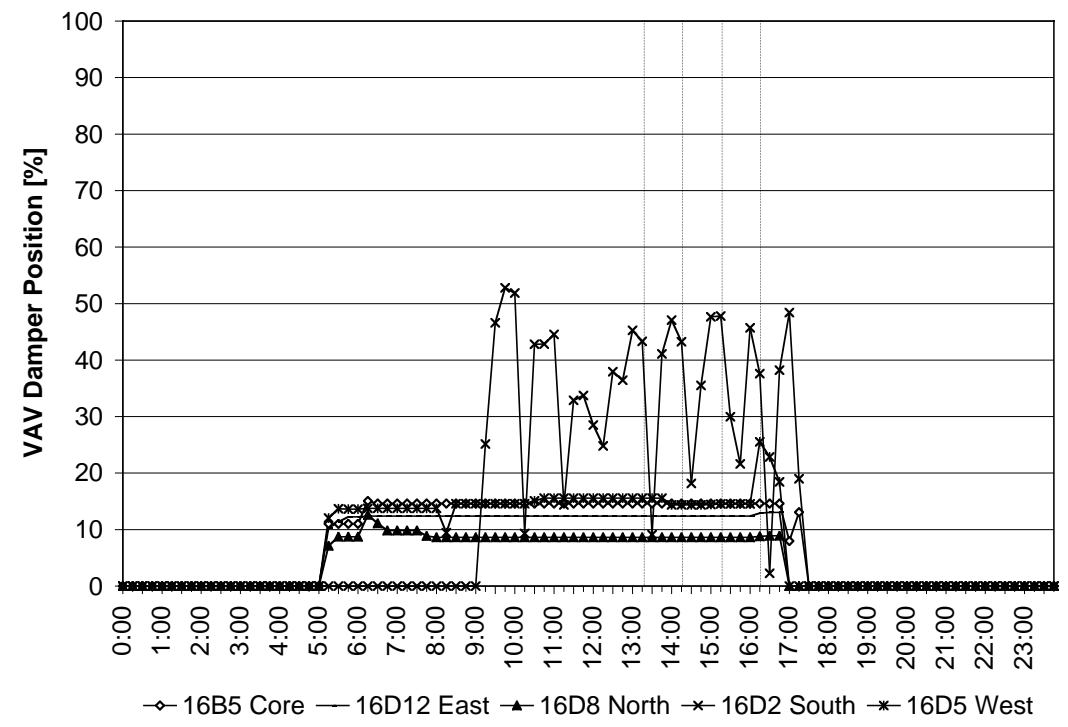

Figure VII-24. GSA - North 16th Floor VAV Damper Position 
The VAV damper positions on the South 4th floor were not affected by the price signal (Figure VIII-24).

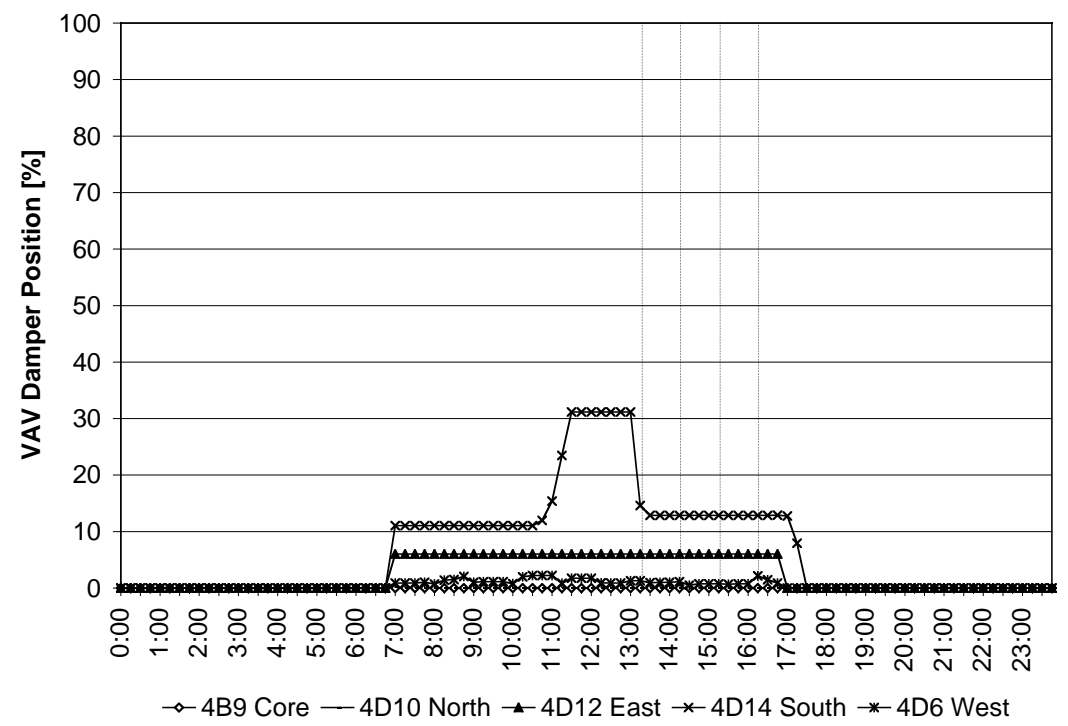

Figure VII-25. GSA - South 4th Floor VAV Damper Position

The south-side zone (16D11) VAV damper on South 16th floor changed its position to maintain the zone temperature setpoint which was changed based on the price signal. The core zone (16B3) damper position seems reduced due to the zone setpoint increase.

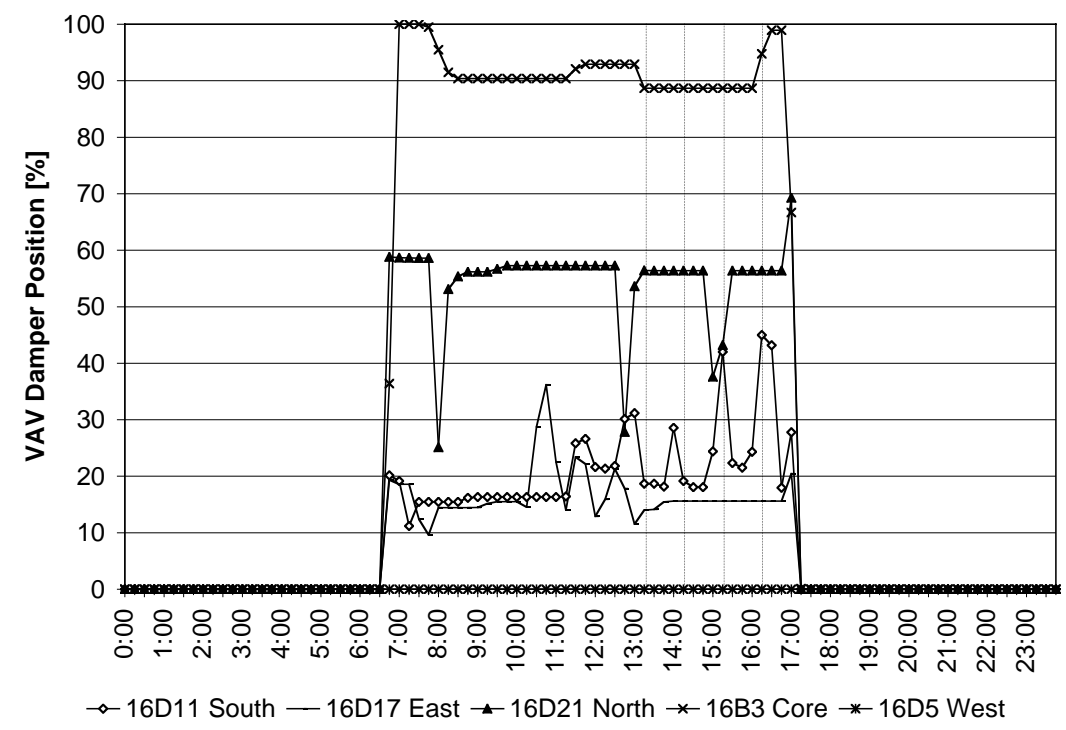

Figure VII-26. GSA - South 16th Floor VAV Damper Position 


\section{Appendix VII-4. Roche - Additional Figures}

\section{Individual Building Power and Baseline}

OAT regression model baselines were developed for each building at the Roche site. Building A2 fan power savings could be clearly identified in the whole building power. The standard error range shows the building might have had irregular nighttime operations over the previous days.

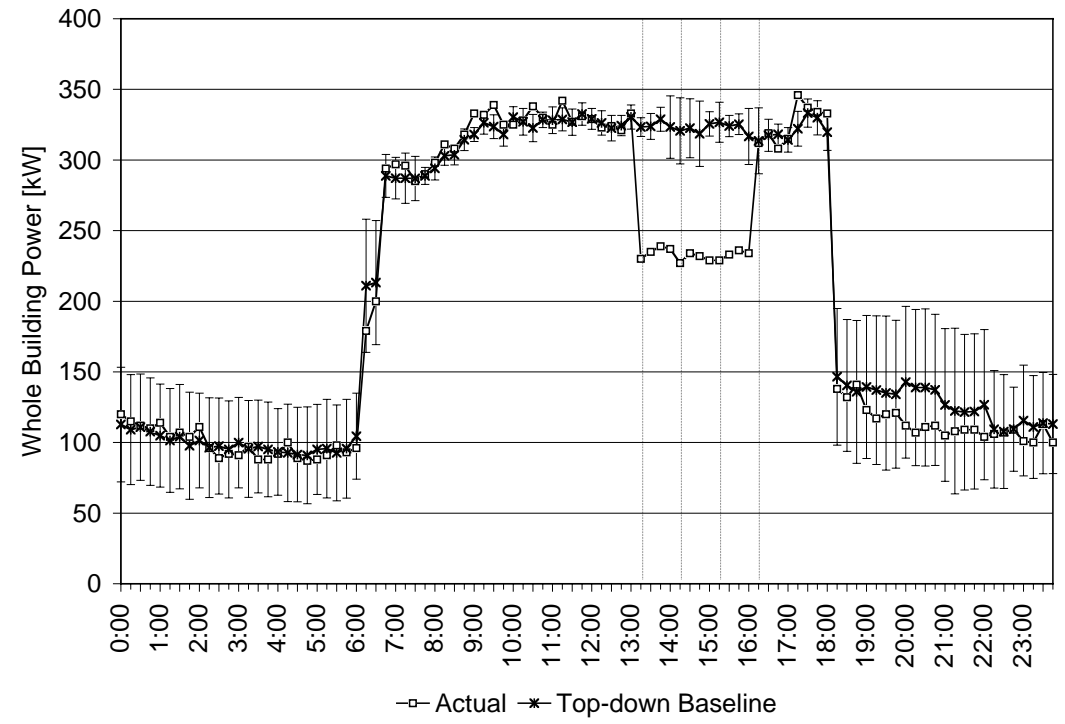

Figure VII-27. Roche - Building A2, Whole Building Power and Baseline

The Building SS operational pattern was changed on October $30^{\text {th }}$, and the demand reduced approximately $50 \mathrm{~kW}$ during the afternoon (Figure VIII-27). The regression model shows higher demand than the actual demand seen on the test day because the model is affected by the demand prior to October $30^{\text {th }}$. 


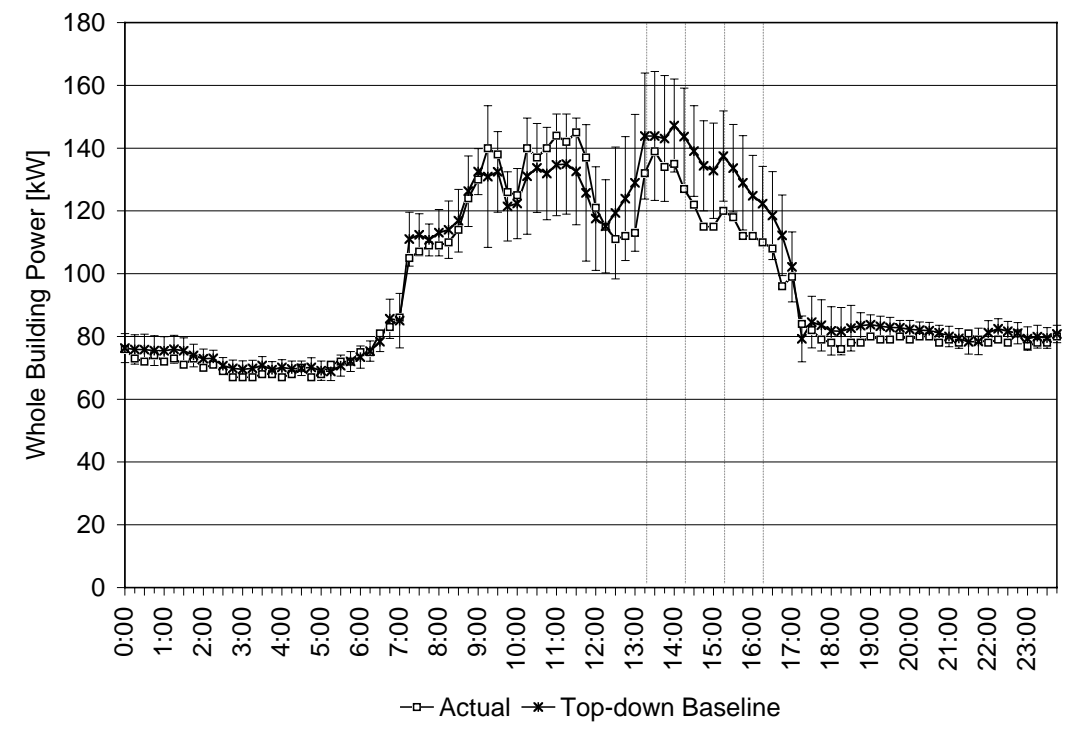

Figure VII-28. Roche - Building SS, Whole Building Power and Baseline

Building FS' power demand fluctuated because of electric cooking appliance use (Figure VIII-27). The spiking demand was averaged in the baseline model. Clear demand savings were obtained during the $\$ 0.75 / \mathrm{kWh}$ period.

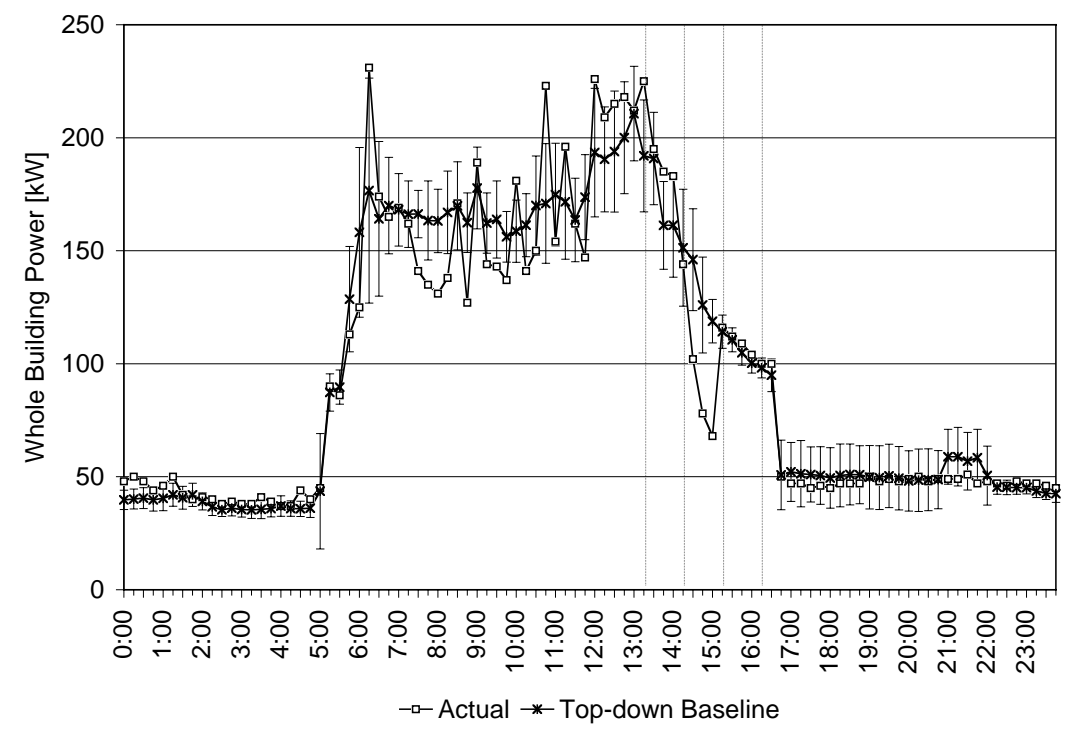

Figure VII-29. Roche - Building FS, Whole Building Power and Baseline 


\section{Appendix VII-5. UCSB - Additional Figures}

\section{Components Power and Baseline}

Cooling power dropped significantly at the beginning of the $\$ 0.75 / \mathrm{kWh}$ period because of the cooling valve close strategy (Figure VIII-29). However, the cooling power demand spiked up at the end of the shed period and was greater than the baseline demand for that time period.

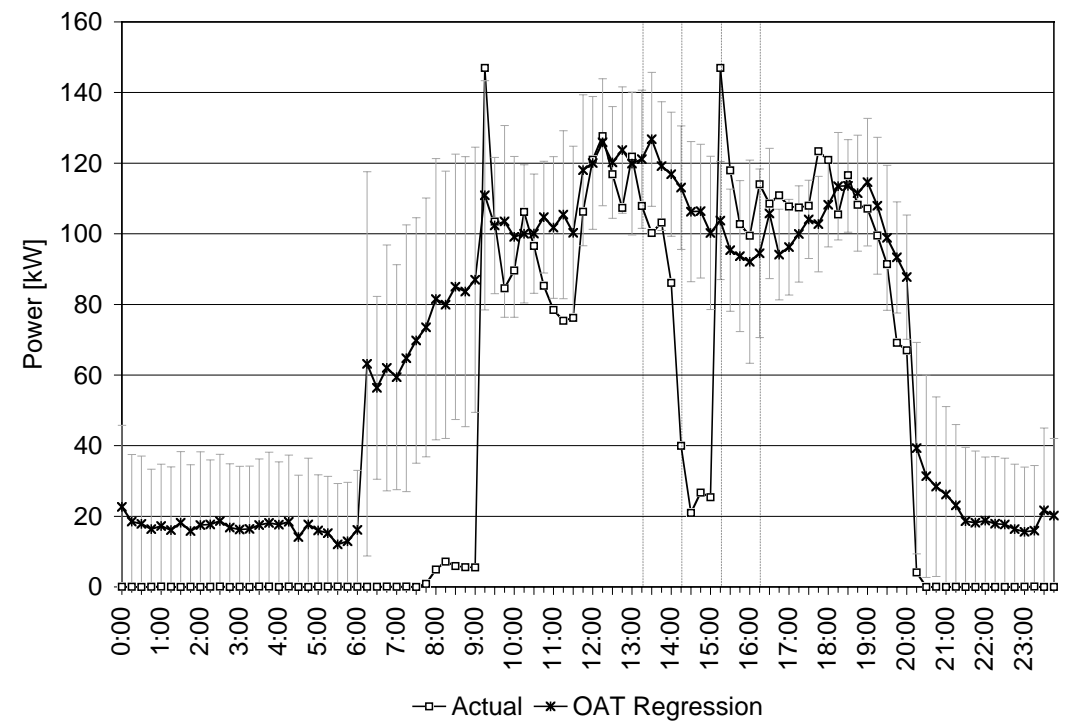

Figure VII-30. UCSB - Cooling Power and OAT Regression Baseline

Although an OAT regression model was developed for the fan power, the predicted demand from the model was much lower than the actual demand around the shed period (Figure VIII-30). An alternative baseline was developed for use in determining the fan power demand savings. 


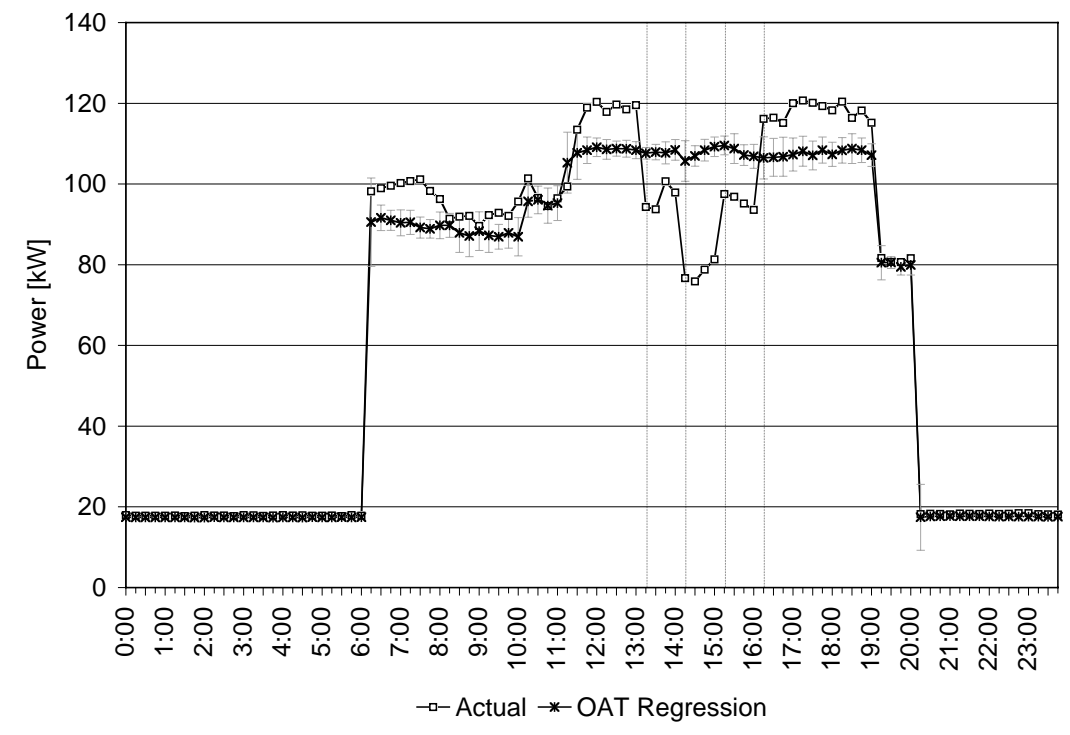

Figure VII-31. UCSB - Fan Power and OAT Regression Baseline

All of the supply fans have VFD but operated at constant volume except for the Library III - Supply Fan \#2, which was operated in variable load. Because of this, the OAT regression model was applied only to this fan. Although this fan was assigned to limit the VFD\% based on the price signal, it didn't change the operation. The fan power shows that the demand spiked twice during the shed period (Figure VIII-31).

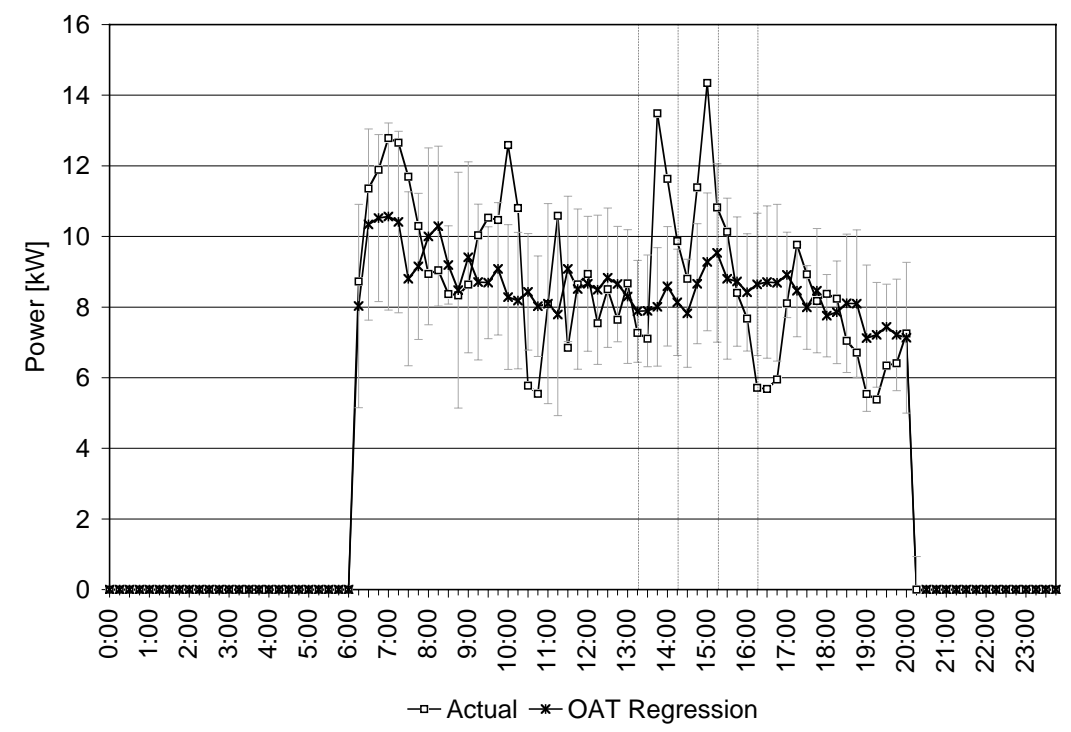

Figure VII-32. UCSB - 3S2-SF Demand and OAT Regression Baseline 


\section{$\underline{\text { Zone Temperatures }}$}

Most zones in the library didn't show noticeable temperature changes during the shed (Figure VIII-32 through VIII-34). Several zone temperatures at Library II increased two to three degrees $\mathrm{F}$ when the cooling valve was closed.

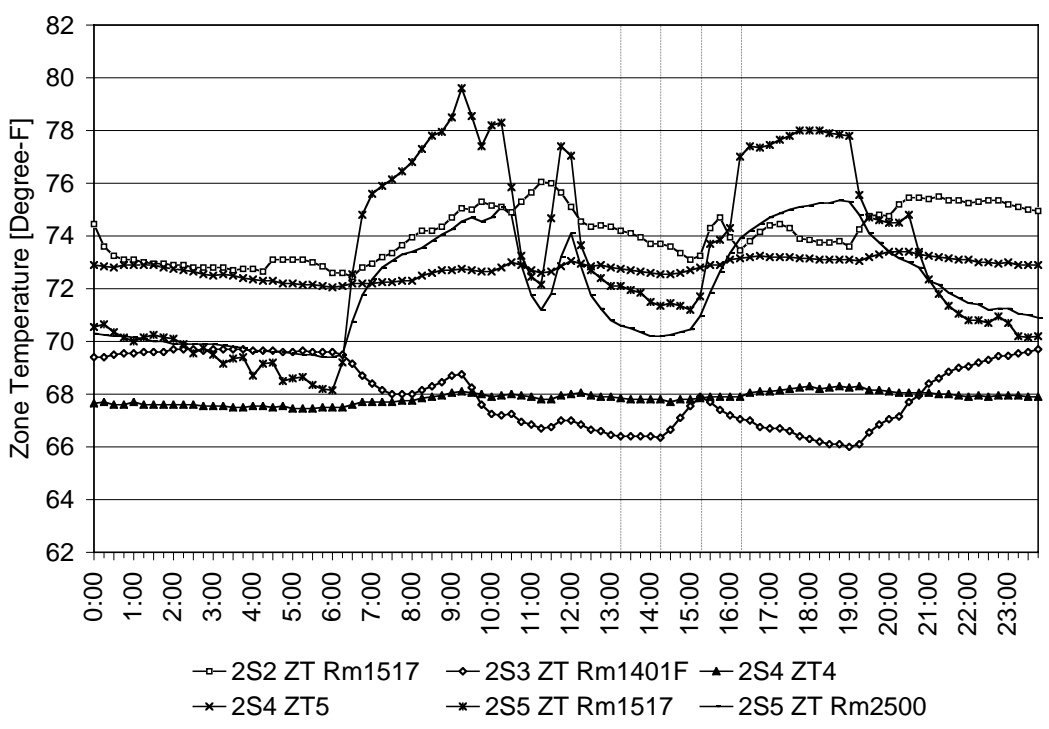

Figure VII-33. UCSB - Library II, Zone Temperatures

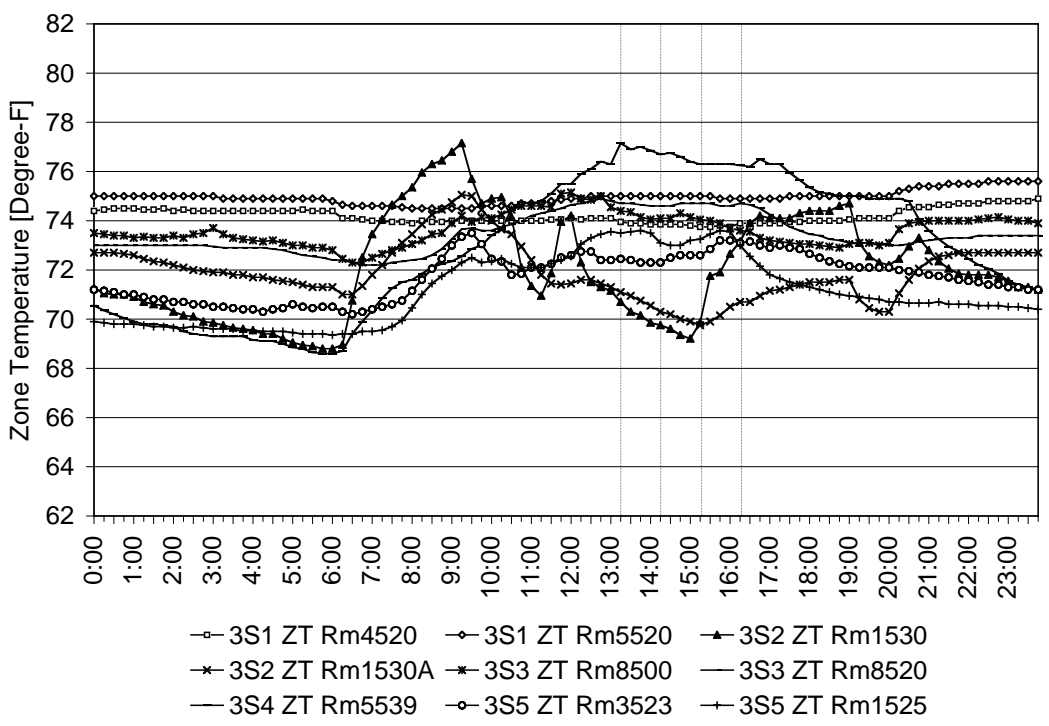

Figure VII-34. UCSB - Library III, Zone Temperatures 


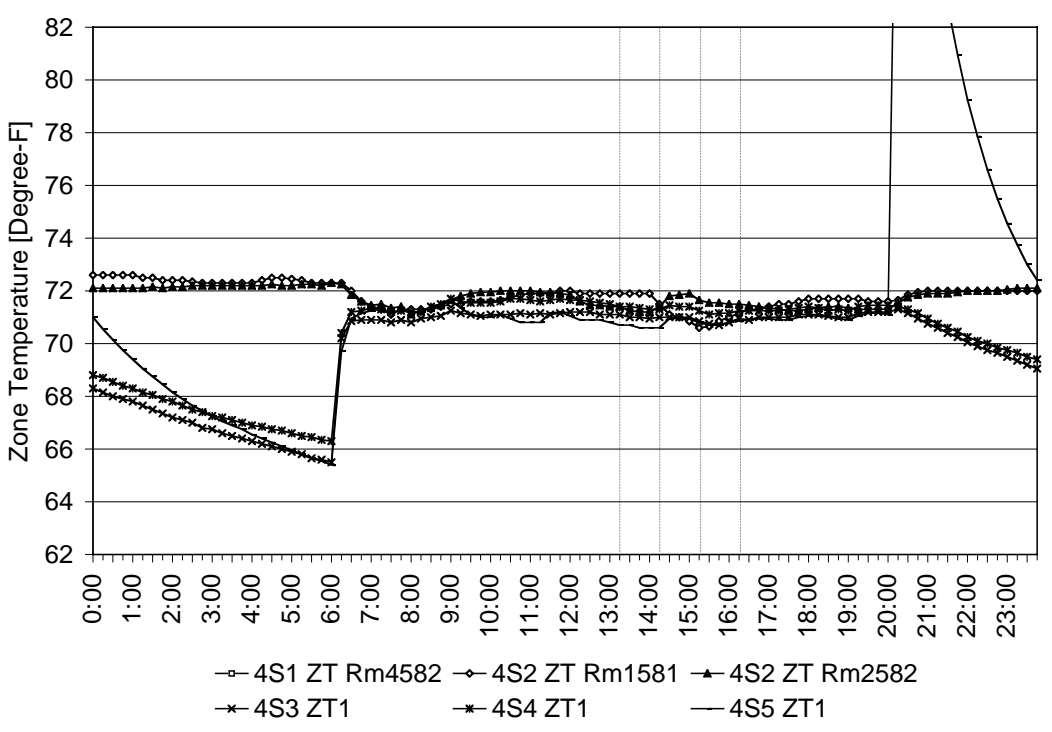

Figure VII-35. UCSB - Library IV, Zone Temperatures 


\title{
Appendix VIII. Previous DR Participation and Site Contact
}

\author{
Appendix VIII-1.Prior Demand Response Program Participation
}

One of the selection criteria for participation in the LBNL tests was that each site must have demonstrated a DR capability through some DR program. Table VII-1 summarizes the programs participated, size of the curtailment capability—estimated and demonstrated.

Table VIII-15. Demonstrated Demand Response Capability of the Test Participants

\begin{tabular}{|c|c|c|c|c|c|}
\hline \multicolumn{6}{|c|}{ Sites for final test } \\
\hline & Facility Type & $\begin{array}{l}\text { Estimated Curtailment } \\
\text { Capability (1) }\end{array}$ & $\begin{array}{l}\text { Demonstrated Curtailment } \\
\text { Capability (1) }\end{array}$ & $\begin{array}{l}\text { Pilot Test Date } \\
\text { (1) }\end{array}$ & Participation into DR Programs \\
\hline Albertsons & Supermarket & $6.8 \mathrm{MW}$ for 264 sites & $5.4 \mathrm{MW}$ for 264 sites & 10/29/2001 & $\begin{array}{l}\text { CEC Peak Load Reduction Program---Demand } \\
\text { Responsive Building Systems Element } \\
\text { (SB5X): Subelement } 2\end{array}$ \\
\hline BofA & $\begin{array}{l}\text { Low-rise Office } \\
\text { Building }\end{array}$ & 2.8MW for 78 sites(2) & $\begin{array}{c}2.2 \mathrm{MW} \text { for } 78 \text { sites, } 2.4 \mathrm{MW} \text { for } \\
81 \text { sites ( } 3 \text { sites added in } 2003 \text { ) } \\
\text { (3) }\end{array}$ & & $\begin{array}{l}\text { CEC Peak Load Reduction Program---Demand } \\
\text { Responsive Building Systems Element } \\
\text { (SB5X): Subelement } 3 \text {. WebGen was the } \\
\text { contractor for the award. }\end{array}$ \\
\hline $\begin{array}{l}\text { GSA-Oakland } \\
\text { Federal } \\
\text { Building } \\
\end{array}$ & Office & $800 \mathrm{~kW}$ & $\begin{array}{c}800 \mathrm{~kW} \text { (shed coordinated with } \\
\text { SF Federal building, total of } 1.2 \\
\text { MW from } 2 \mathrm{M} \text { sqft) }\end{array}$ & & $\begin{array}{l}\text { Performed during the CAISO DRP Event on } \\
\text { July } 3,2001 \text {. }\end{array}$ \\
\hline UCSB & $\begin{array}{l}\text { University } \\
\text { Campus } \\
\text { Building } \\
\end{array}$ & $1 \mathrm{MW}$ & $1.1 \mathrm{MW}$ & $7 / 28 / 2001$ & $\begin{array}{l}\text { CEC Peak Load Reduction Program---Demand } \\
\text { Responsive Building Systems Element } \\
\text { (SB5X): Subelement 2. Performed during the } \\
\text { CAISO DRP Event on July 3, } 2001 \text {. }\end{array}$ \\
\hline \begin{tabular}{l|} 
Roche \\
Pharmaceutica \\
Is
\end{tabular} & $\begin{array}{l}\text { Manufacturing } \\
\text { Facility } \\
\end{array}$ & 1.2 MW for 21 sites & $2.5 \mathrm{MW}$ for 21 sites & 9/28/2001 & $\begin{array}{l}\text { CEC Peak Load Reduction Program--Demand } \\
\text { Responsive Building Systems Element } \\
\text { (SB5X): Subelement 2. Also participated in the } \\
\text { "Cash for Kilowatts" Program. ( Offered } \\
\text { 1.5MW within 20-minutes and delivered } \\
\text { 2MW.) }\end{array}$ \\
\hline \multicolumn{6}{|l|}{$\begin{array}{l}\text { Sites } \\
\text { considered but } \\
\text { not in final test }\end{array}$} \\
\hline USPS & $\begin{array}{l}\text { Mail Distribution } \\
\text { Center }\end{array}$ & 4.76 MW for 24 sites & $\begin{array}{l}\text { The test during Summer } 2002 \\
\text { was not successful. }\end{array}$ & May-02 & $\begin{array}{l}\text { CEC Peak Load Reduction Program---Demand } \\
\text { Responsive Building Systems Element } \\
\text { (SB5X): Subelement } 2\end{array}$ \\
\hline
\end{tabular}

\section{Albertsons}

Albertsons participated in the Peak Load Reductions Program-Demand Responsive Buildings System Element as a Grantee. Albertsons participated to this program with 264 of their supermarket facilities in California. They estimated a DR capability of 6.8 MW and they were able to demonstrate load reductions of 5.4 MW at these facilities during the pilot test on October 29, 2001. The response mainly comes from reductions in anti-sweat heater energy and case lighting.

\section{Bank of America}

Bank of America participated in the Peak Load Reductions Program-Demand Responsive Buildings System Element with WebGen as the Small Commercial Contractor. Bank of America participated to this program with 81 of their facilities in California. They estimated a DR capability as $2.8 \mathrm{MW}$ (for the initial 78 facilities-3 were added to the set in 2003) and they were able to demonstrate load reductions of 2.2 MW for the 78 original facilities (2.4 MW for the 81 facilities). The response mainly came from HVAC measures. 


\section{Oakland Federal Building (GSA) and 450 Golden Gate, SF (GSA)}

The Oakland Federal Building (Ronald Dellums Federal Building) participated in the California Independent System Operator's (ISO) 2001 Summer Demand Reduction Program (DRP) together with the Philip Burton Federal Building in San Francisco (450 Golden Gate, SF). The demand reduction target for these two buildings was 1.2 MW. Only one curtailment (July 3, 2001) was called during the program duration (June 1 to September 30). According to ISO, $780 \mathrm{~kW}$ (65\% of the target) was shed. However, the baseline calculation used by the ISO does not take into account the effect of temperature variation on energy use in commercial buildings. LBNL estimated that if a baseline model that accounted for temperature was used, the calculated shed would have been $120 \%$ of the target.

\section{University of California, Santa Barbara}

UCSB participated in the California Independent System Operator's (ISO) 2001 Summer Demand Reduction Program (DRP). Infotility was the load aggregator facilitating the participation. July $3^{\text {rd }}$ was the only DRP event that summer. The results of the Demand Relief Program (DRP) implementation for July $3^{\text {rd }}, 2001$ from 2:00 p.m. to 6:00 p.m. show that the demand reduction was $60 \%$ of the target of $1 \mathrm{MW}$. The ISO calculation for reduction is based on the previous 10 normal, non-curtailed weekdays. This baseline calculation was revised because it is not adjusted for weather conditions. July $3^{\text {rd }}$ was an abnormally hot day compared to the previous weeks. The average cooling degree-days per day for the ten-day baseline was 3.7 CCD versus 6.2 CCD for July $3^{\text {rd }}$. Using the temperature-adjusted baseline, the demand reduction is calculated to be $1.1 \mathrm{MW}$.

UCSB also participated in the Peak Load Reductions Program-Demand Responsive Buildings System Element as a Grantee. They estimated a DR capability of 1MW and they were able to demonstrate load reductions of 1.1 MW at these facilities during the pilot test on July $28^{\text {th }}, 2001$.

\section{Roche Pharmaceuticals}

Infotility has been working with the site in a PIER research project to test communications capabilities of the Infotility XML electricity price server. Roche participated in the CEC "Cash for Killowatts" program. They offered a reduction of $1.5 \mathrm{MW}$ within the 20 -minute window that is required by the program. They were able to curtail more than $2 \mathrm{MW}$ within 20-minutes of request. This qualified them for a significant grant from CEC. In addition to this they received multiple rebates from local energy provider (City of Palo Alto) for demand limiting. Three buildings at the site were chosen for the demonstration. The building chosen are A2 (administration, auditorium $101,078 \mathrm{ft}^{2}$ ), SS (facility management office $67,862 \mathrm{ft}^{2}$ ), and FS (cafeteria $23,159 \mathrm{ft}^{2}$ ). The central plant located in the campus provides cooling energy to the buildings. Laboratory buildings were avoided for the demonstration because their airflow and indoor air quality are more carefully controlled. The buildings chosen are non-critical areas where are expected to have relatively fewer complaints.

Roche participated in the Peak Load Reductions Program-Demand Responsive Buildings System Element as a Grantee. Roche participated to this program with 21 of their facilities in California. They estimated a DR capability of 1.2 MW and they were 
able to demonstrate load reductions of $2.5 \mathrm{MW}$ at these facilities during the pilot test on September $28^{\text {th }}, 2001$.

\section{United States Postal Service (USPS)}

USPS participated in the Peak Load Reductions Program-Demand Responsive Buildings System Element as a Grantee. USPS participated to this program with 24 of their facilities in California-mostly distribution centers. They estimated a DR capability of 4.76 MW. The pilot tests of May 2002 were not successful primarily because the test days were cool and there was minimal cooling demand. The main DR response in these facilities was planned reduction of chiller power mainly by reducing the service level at the facility. 


\section{Appendix VIII-2. Interaction with Site Contacts}

\section{Albertsons}

LBNL first contacted Albertsons Supermarkets in February, 2003 in an effort to recruit them to participate in the DR test. Our negotiations took many months. LBNL's communications with them was delayed by the time required to finalize the XML signal specifications and associated software requirement. Another reason for the delay is the complicated corporate structure. We tried to contact Invensys to secure the technical development for the communications. We later found out that Elutions was fully acquired by Engagenet and we needed to work with them. Our main contact at Engagenet resigned during our lengthy discussions with them leaving us where we started. During our effort to secure a site we worked with Albertsons, Engagenet, Elutions, and the Albertsons local management.

During September and October activities were in two areas:

(1) We worked on securing a specific site, convincing the store management that the tests would not be too disruptive and working with the store management to develop a two level response strategy. The building selected for the test is 3000 East $9^{\text {th }}$ St. in Oakland. The first site that was nominated for the test was not a good site because the whole-building electric meter was not connected to the EIS. We also needed to work with Albertsons in making hardware modifications at the site to facilitate the communication between EIS and the controls.

(2) We worked with Elutions/Engagenet for the connectivity and communication between Infotility and the EIS. For this, we needed to procure services from Infotility too.

\section{Bank of America}

LBNL has been in communication with WebGen for more than one and a half years. WebGen staff visited LBNL while LBNL was conducting a review of Energy Information Systems and we have been in several meetings where they demonstrated their products to us. Through WebGen, we contacted Jones Lang LaSalle (JLL) who is the property manager for many of the Bank of America (B of A) buildings in California. We had a meeting with JLL in early March 2003 where we identified possible buildings. Bank of America was very motivated to participate in our test.

Bank of America has 78 buildings that have DR capability facilitated by WebGen. Most of the buildings are branch offices. There are four buildings that are not branch offices and they vary in size form $100,000 \mathrm{ft}^{2}$ to $500,000 \mathrm{ft}^{2}$. As a result of our meetings with B of A, we identified the 500,000- $\mathrm{ft}^{2}$ building in San Francisco as our primary candidate. This San Francisco building is equipped with a Honeywell Energy Management Control System (EMCS). As mentioned above, the EIS on top of this system is WebGen. The San Francisco office was Webgen's first experience connecting to a Honeywell system. We were initially concerned that this may pose a problem because when the building was recruited for the project, the gateway connectivity had not been completed. During 
August, 2003 we were informed that the connectivity was completed and the testing could proceed.

In early September, 2003, JLL and Webgen informed us that the San Francisco office building was being sold. Webgen and JLL selected the B of A's Concord Technology Center (CTC) for the study. The CTC consists of 4 large buildings with about 1 million $\mathrm{ft}^{2}$ of space. Webgen links to a Trane Trace EMCS, and the linkage has been completed. We visited the site on September 10. During this meeting one of the four buildings (Building B) was selected as the site that would be monitored. The response strategy, the monitoring and verification methodology, and other issues related to communications were discussed.

During the following weeks, we were in contact with the building operators. We worked with them during the installation of our short term metering equipment. The building operators also provided us with data from the Trane system to complement data from Webgen and our own short term metering.

During late September and October WebGen completed software upgrade to accept the XML signals from Infotility, submetering was installed to verify our ability to measure the shed strategy, the list of Trane data points to be monitored were identified and LBNL obtained Webgen data files to ensure we can analyze the test results.

Bank of America, JLL, and WebGen chose a site in Concord which has four buildings (A, B, C and D) used for office and data center. Building D is a data center, and has the central cooling plant which supplies chilled water to all the four buildings. The other buildings are offices. Building B was chosen for the test, because it had no known problem such as over heating or solar gain problems.

\section{Oakland Federal Building (GSA) and 450 Golden Gate, SF (GSA)}

LBNL has been working with this building for several years. One project consisted of an energy assessment using EMCS trend logs, which resulted in a paper at the National Conference on Building Commissioning. A second study was conducted to assist in the development of the DR strategies. LBNL is also partnering with GSA on the development of GEMnet: GSA Energy and Maintenance Network. LBNL's involvement in this site build's on the multi-year relationship and collaboration with GSA and the onsite staff.

\section{University of California, Santa Barbara}

UCSB was the subject of an LBNL study on the cost effectiveness of Energy Information Systems (Motegi et al, 2003). This study involved interviewing Jim Dewey, the energy manager, on how he uses the EIS. Multi-year energy data were collected and specific examples of building operating problems identified with the EIS. 


\section{Roche Pharmaceuticals}

\section{United States Postal Service (USPS)}

LBNL has been working with USPS through funding from Federal Energy Management Program (FEMP) for almost a decade. The Electricity Markets and Policy Group at LBNL have been leading this activity to help USPS strategize energy procurement and energy-efficiency investments.

Before the DR tests during 2002, we were invited to a training session facilitated by Viron for USPC facility managers and we had a chance to meet several of the key people in this organization. At this meeting, we had a chance to introduce our project both to Viron and USPS.

In order to convince USPS to participate in our test, we offered them to provide an estimate of their potential savings under the new proposed CPP tariff. USPS provided us with real time interval data for all of their facilities. Because only PG\&E provided prices on their proposed CPP tariff, we analyzed the potential savings for the West Sacramento Distribution Center that is supplied by PG\&E. Unfortunately our analysis did not show significant savings - especially with the delayed start day of the CPP tariff. Table VI-2 indicates that if the tariff had been available, it would be beneficial for the facility to participate. Even without any action, their gain would be around $\$ 6000$. With a response of about $5 \%$, they could have increased this benefit to about $\$ 9000$. However, these profits would diminish and serious losses would take place with a delay of the program to June.

Table VIII-16. The benefits of participating in CPP tariff for a USPS distribution center in West Sacramento using load profiles from 2002(\$)

\begin{tabular}{|l|c|c|c|c|c|c|c|c|}
\cline { 5 - 9 } \multicolumn{1}{l|}{} & May & June & July & Aug & Sept & Oct & \multicolumn{2}{c|}{ Total } \\
\cline { 5 - 10 } & & & & & & & June to Oct & May to Oct \\
\hline $\begin{array}{l}\text { No Action } \\
\text { days duariment on CPP } \\
\text { hours }\end{array}$ & 18153 & 16794 & -14676 & -32926 & 924 & 17445 & -12440 & 5714 \\
\hline
\end{tabular}

Note: CPP days were selected as the 12 days with the highest California ISO system load in 2002

In a further attempt to secure their participation, we contacted Viron to explore the possibility of synchronizing our tests with the tests USPS/Viron needed to perform during the summer as part of the Peak Load Reduction Program. At that point, Viron was very motivated to incorporate the software developed by Infotility in their system to receive XML price signals. We provided Viron with the preliminary specification of the XML signal and their technical group began working on this issue. Viron was going to help us pick the most suitable site out to the 24 .

Because of LBNL's long relation with USPS, we have extensive information on each site. As mentioned, we also were given access to interval near-real-time data on all of their facilities that are of interest to us. LBNL made great progress in discussions with USPS and Viron. A postal distribution center in San Jose was selected for the test 
because the on-site staff was supportive and interested in DR. Numerous discussions were made with USPS and the MOU was signed.

During late summer, Chevron Energy Services purchased Viron. We continued numerous discussions with Bruce Dickenson, our contact at Viron. However, we were unable to proceed with the project because Chevron was upgrading the Information Technology infrastructure. They are unable to make the additional programming upgrades to the Utility Vision system at a time when the infrastructure was changing. Although USPS and Viron dropped out of the test, they are still interested in participating in any future projects. 


\section{Appendix VIII-3.Problems Encountered During Test Period}

Several problems were encountered during the two-week test period, specifically before the initialization of the test on November $12^{\text {th }}$ as well as before the November $19^{\text {th }}$ test. During the two three-hour high price periods, signaling went smoothly except for a few minor problems. Table 4-2 summarizes the kinds of problems encountered.

Table VIII-17. Problems Encountered During The 2-Week Test Period

\begin{tabular}{|l|c|c|c|c|c|}
\hline & Albertsons & B of A & GSA & Roche & UCSB \\
\hline Tuned into wrong channel & $\checkmark$ & $\checkmark$ & & & \\
\hline Not confirming & $\checkmark$ & & $\checkmark$ & & \\
\hline Signaled unsuccessful data transfer & & & & & $\checkmark$ \\
\hline Time stamp discrepancies & & & $\checkmark$ & & \\
\hline $\begin{array}{l}\text { Programmed delay in } \\
\text { confirmation signal }\end{array}$ & & & $\checkmark$ & & \\
\hline $\begin{array}{l}\text { Action premature } \\
\text { (before price in effect) }\end{array}$ & $\checkmark$ & $\checkmark$ & $\checkmark$ & $\checkmark$ & $\checkmark$ \\
\hline $\begin{array}{l}\text { Signal confirmed > 2 minutes } \\
\text { from time published }\end{array}$ & & & $\checkmark$ & & \\
\hline
\end{tabular}

All of these problems are start-up problems and could be eliminated with proper commissioning. We do not anticipate that these will cause long term issues and/or bottlenecks. The rest of this section provides a more detailed accounting of these problems. One issue to note is that all of these problems were resolved before the second Auto-DR test on November $19^{\text {th }}, 2003$.

\section{Albertsons}

Polling wrong channel._A private test channel was set-up for each site for use prior to the official test window. During the official test window, the sites switched over from their private test channel to the official LBNL "production" channel. Albertsons polled their private test channel in addition to the production channel until November $12^{\text {th }}$ at 12:45 pm. LBNL observed this discrepancy and the problem was corrected minutes before the first Auto-DR test.

Not Confirming. During the two-week test period, 110 signals were not confirmed. The signal was not confirmed once at $10: 30 \mathrm{pm}$ on November $13^{\text {th }}$. Confirmation stopped totally between November $17^{\text {th }}$ at 4:15 p.m. and November $19^{\text {th }}$ at 9:00 am. See Table 43 for part of this time period's communication log. Corrections were made just before the second Auto-DR test. 
Table VIII-18. Signal Not Being Confirmed - Albertson

\begin{tabular}{|c|c|c|c|c|c|c|c|c|}
\hline ChannelID & $\begin{array}{l}\text { Channel } \\
\text { Description }\end{array}$ & $\underline{\text { UserID }}$ & UserName & $\begin{array}{l}\text { When } \\
\text { requested } \\
\text { by user }\end{array}$ & $\frac{\text { Timestamp }}{\underline{\text { [asc] }}}$ & $\begin{array}{l}\frac{\text { Price }}{\text { sent }} \\
\text { by } \\
\text { server }\end{array}$ & $\begin{array}{l}\frac{\text { Price }}{\text { returned }} \\
\text { by user } \\
\end{array}$ & $\begin{array}{l}\text { When } \\
\text { returned } \\
\text { by user }\end{array}$ \\
\hline 1233 & Price_LBNL1 & 397 & albertsons,cpu1_ & $\begin{array}{l}11 / 19 / 2003 \\
8: 47: 10 \\
\text { AM } \\
\end{array}$ & $\begin{array}{l}\text { 11/19/2003 } \\
9: 00: 00 \\
\text { AM }\end{array}$ & 0.1 & & \\
\hline 1233 & Price_LBNL1 & 397 & albertsons,cpu1_ & $\begin{array}{l}11 / 19 / 2003 \\
9: 02: 09 \\
\text { AM }\end{array}$ & $\begin{array}{l}11 / 19 / 2003 \\
9: 15: 00 \\
\text { AM }\end{array}$ & 0.1 & 0.1 & $\begin{array}{l}\text { 11/19/2003 } \\
9: 20: 27 \\
\text { AM }\end{array}$ \\
\hline
\end{tabular}

\section{Bank of America}

Polled wrong channel and premature action. The Bank of America site tested their systems on the afternoon of November $1^{\text {st }}$. Although the test started at 6:00 pm on November 11th they did not start to listen to any channel until 7:00 am on November $12^{\text {th }}$. At that point they started sending requests to their own test channel and not the LBNL-channel. The problem was corrected at 8:30 am on November $12^{\text {th }}$ when they started requesting to the proper test channel.

\section{GSA}

Not confirming, time stamp discrepancies, programmed delay in confirmation signal, action premature and signal confirmed $>2$ minutes. On three separate days over the two week test period, a total of 35 signals were not confirmed by the GSA system: November $11^{\text {th }}(9: 45$ am to $5: 30 \mathrm{pm})$, November $12^{\text {th }}(1: 45 \mathrm{pm}$ when the signal switched to $\$ 0.30 / \mathrm{kWh}$ and $3: 15 \mathrm{pm}$ when the signal was $\$ 0.75 / \mathrm{kWh})$, November 18 (10:15am), and November 21st (11:00 am). At the beginning of the two week test period, the GSA confirmation stamp was about an hour later than the time stamp of the signal and receipt time stamps. This problem was due to the time difference between the different servers involved and was corrected on November $11^{\text {th }}$ at 5:30 $\mathrm{pm}$. Signals were confirmed in greater than two minutes from the time they were published, until approximately the time when the price took effect-13 to 15 minute delay from the time the signal was received.

\section{Roche}

No problems were noticed during the two-week test period. Signals were requested in less than two minutes from the time they were published. Signals were confirmed within two minutes from the time they were requested.

\section{$\mathrm{UCSB}$}

Thirteen Unsuccessful data transfer and action premature. Signals were received incorrectly during the two week test period, some on November $10^{\text {th }}$ and some on 
November $11^{\text {th }}$ (See Table 4-4). The problem was corrected by 11:00 am on November $11^{\text {th }}$.

Table VIII-19. Participant Returning Wrong Value for the Signal - UCSB

\begin{tabular}{|c|c|c|c|c|c|c|c|c|}
\hline ChannelII & $\begin{array}{l}\text { Channel } \\
\text { Description }\end{array}$ & UserID & UserName & $\begin{array}{l}\frac{\text { When }}{\text { requested }} \\
\text { by user }\end{array}$ & $\frac{\text { Timestamp }}{\underline{\underline{\text { asc }]}}}$ & $\frac{\frac{\text { Price }}{\text { sent }}}{\frac{\text { by }}{\text { server }}}$ & $\frac{\frac{\text { Price }}{\text { returned }}}{\text { by user }}$ & $\begin{array}{l}\text { When } \\
\text { returned } \\
\text { by user }\end{array}$ \\
\hline 1233 & Price_LBNL1 & 385 & ucsb,cpu1_ & $\begin{array}{l}11 / 10 / 2003 \\
9: 30: 43 \\
\text { AM }\end{array}$ & $\begin{array}{l}\text { 11/10/2003 } \\
9: 45: 00 \mathrm{AM}\end{array}$ & 0.1 & -1 & $\begin{array}{l}\text { 11/10/2003 } \\
9: 46: 05 \\
\text { AM }\end{array}$ \\
\hline 1233 & Price_LBNL1 & 385 & ucsb,cpu1_ & $\begin{array}{l}\text { 11/10/2003 } \\
9: 46: 05 \\
\text { AM }\end{array}$ & $\begin{array}{l}\text { 11/10/2003 } \\
10: 00: 00 \\
\text { AM }\end{array}$ & 0.1 & 0.1 & $\begin{array}{l}\text { 11/10/2003 } \\
9: 47: 07 \\
\text { AM }\end{array}$ \\
\hline
\end{tabular}

